\begin{tabular}{|l|l|}
\hline $\begin{array}{l}\text { 2. To: (Receiving organization) } \\
\text { Distribution }\end{array}$ & $\begin{array}{l}\text { 3. From: (originating Organization) } \\
\text { Nuclear Safety Support }\end{array}$ \\
\hline $\begin{array}{l}\text { 5. Proj./Prog./Dept./Div.: } \\
\text { TWRS }\end{array}$ & $\begin{array}{l}\text { 6. Design Authority/ Design Agent/Cog } \\
\text { Engr.: }\end{array}$ \\
\hline
\end{tabular}

8. Originator Remarks:

\section{Engr.:}

4. Related EDT Ho.:

NA

7. Purchase Order No.: NA

9. Equip./Component Mo.: NA

10. System/Bldg./Facility: TWRS

11. Receiver Remarks: 11A. Design Baseline Document? [] Yes [X] Ho
12. Major Assm. Dwg. Mo.:

NA

13. Permit/Permit Application No.: NA

14. Required Response Date: March 10, 1999

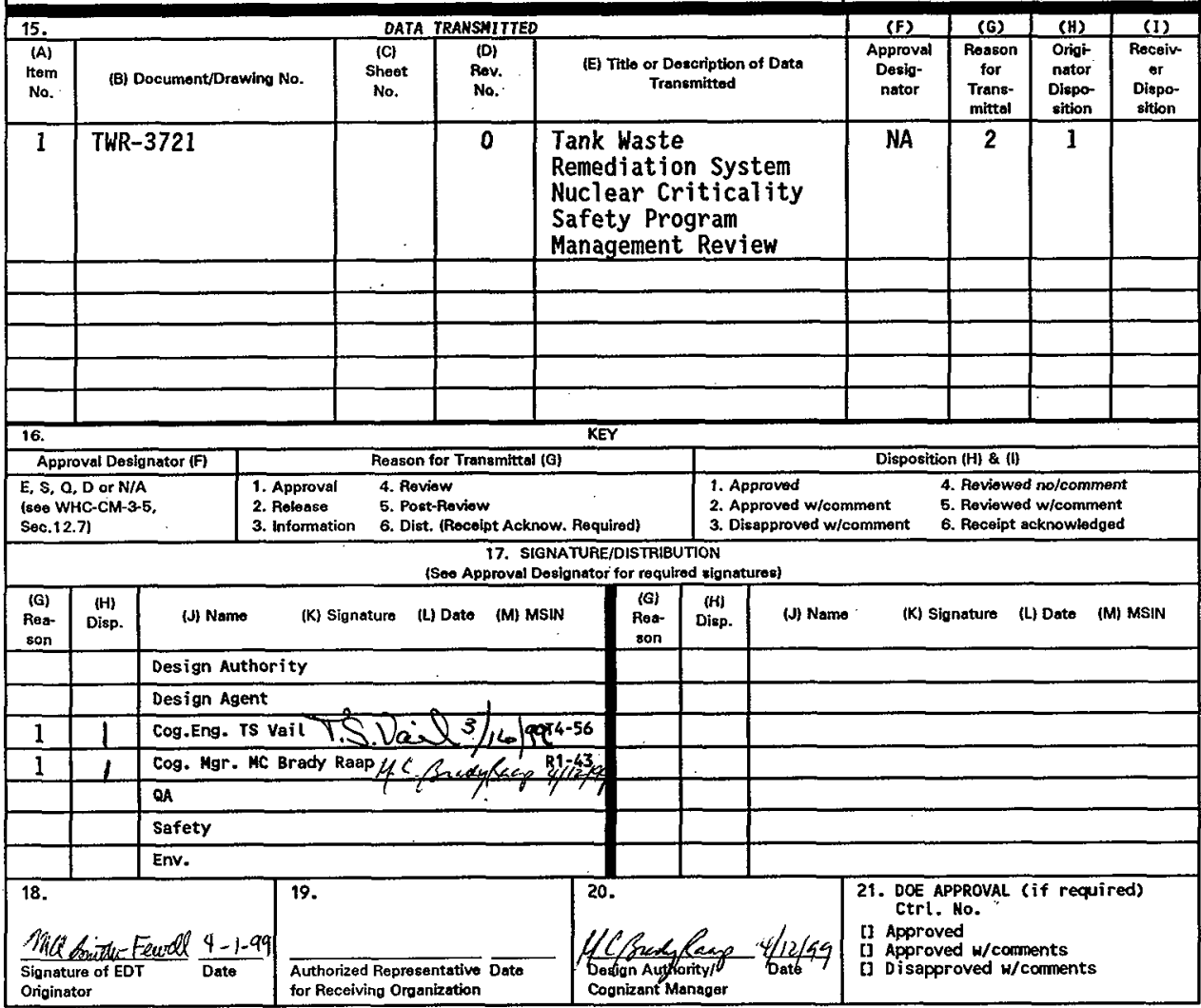




\title{
TANK WASTE REMEDIATION SYSTEM NUCLEAR CRITICALITY SAFETY PROGRAM MANAGEMENT REVIEW
}

M. C. Brady Raap, M. A. Smith-Fewell, D. R. Bratzel \& T. S. Vail Duke Engineering and Services Hanford, Inc.

Richland, WA 99352

U.S. Department of Energy Contract DE-AC06-96RL13200

\author{
EDT/ECN: 625111 \\ UC: .610 \\ Org Code: 2 N500 \\ Charge Code: 101964BB30 \\ B\&R Code: EW3120072 \\ Total Pages: $19 \overline{4}$
}

Key Words:

Management review, TWRS criticality safety, requirements, nuclear

\section{Abstract:}

This document provides the results of an internal management review of the Tank Waste Remediation System (TWRS) criticality safety program, performed in advance of the DOE/RL assessment for closure of the TWRS Nuclear Criticality Safety Issue, March 1994. Resolution of the safety issue was identified as Hanford Federal Facility Agreement \& Consent order (Tri-Party Agreement) Milestone M-40-12, due Septembex 1999.

TRADEMARK DISCLAIMER. Reference herein to any specific commercial product, process, or service by trade name, trademark, manufacturer, or othenwise, does not necessarily constitute or imply its endorsement, recommendation, or favoring by the United States Government or any agency thereof or its contractors or subcontractors.

Printed in the United States of America. To obtain copies of this document, contact: Document Control Services, P.O. Box 950, Mailstop H6-08, Richland WA 99352, Phone (509) 372-2420; Fax (509) 376-4989.
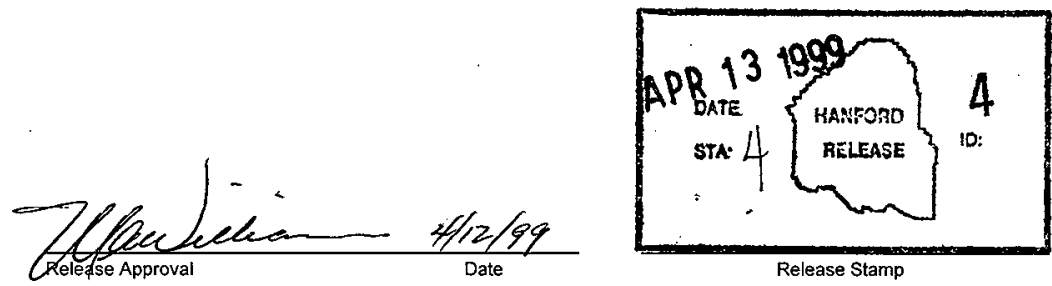

Release Stamp 


\title{
TANK WASTE REMEDIATION SYSTEM NUCLEAR CRITICALITY SAFETY PROGRAM MANAGEMENT REVIEW
}

\author{
Michaele C. Brady Raap \\ Mary Anne Smith-Fewell \\ Terry S. Vail \\ David R. Bratzel \\ DE\&S Hanford, Inc.
}

April 1999 
TWR-3721 Rev. 0

\section{EXECUTIVE SUMMARY}

This document provides the results of an internal management review of the Tank Waste Remediation System (TWRS) criticality safety program, performed in advance of the DOE/RL assessment for closure of the TWRS Nuclear Criticality Safety Issue, which was defined in March 1994. Resolution of the safety issue was identified as Hanford Federal Facility Agreement and Consent Order (Tri-Party Agreement) Milestone M-40-12, with a due date of September 1999.

The safety issue comprises a technical and an administrative part. The technical portion of the safety issue is uncertainty in the amounts and distribution of fissile material in the highlevel waste storage tanks, and was resolved in 1996 (i.e., it is considered closed). The administrative part relates to the need to strengthen the nuclear criticality safety program. Formal closure of the safety issue is contingent on establishing that the administrative criticality safety program at TWRS is sufficient to ensure continued safe operations in support of the TWRS Mission. This review supports closure of the administrative portion of the TWRS Nuclear Criticality Safety Issue by evaluating the TWRS nuclear criticality safety program for compliance with applicable requirements. The primary sources of those requirements include WHC-SD-MP-SRID-001, High Level Waste Storage Tank Farms/242-A Evaporator Standards/Requirements Identification Document, HNF-SD-WM-TSR-006, Tank Waste Remediation System Technical Safety Requirements, and Project Hanford Management System procedures (HNF-PRO documents). The requirements were taken from the source documents that were current in July through September 1998.

Those requirements were screened to determine which were applicable to the TWRS nuclear criticality safety requirements. The applicable requirements were traced to TWRS implementing documents (i.e., administrative procedures) to evaluate compliance with the requirements. Detailed requirements compliance matrixes are included as Appendixes to this document. In addition, personnel qualification records and safety equipment identification documents were reviewed to assess the implementation of requirements applicable to personnel and equipment.

The evaluations of program, personnel, and equipment readiness related to nuclear criticality safety did not identify any issues of noncompliance, but did identify the need for improvement in several areas. Recommended actions were developed to address each area for improvement. 
TWR-3721 Rev. 0

This page intentionally left blank 


\section{TABLE OF CONTENTS}

\section{EXECUTIVE SUMMARY}

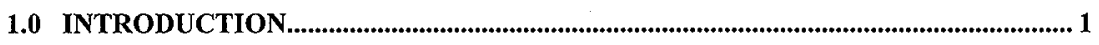

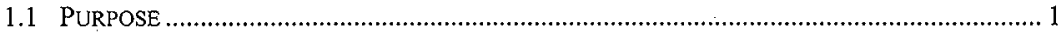

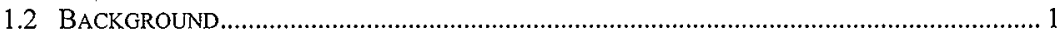

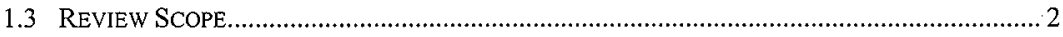

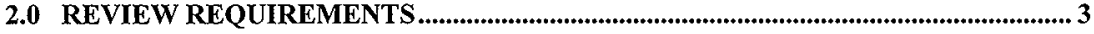

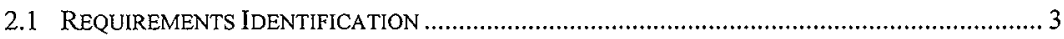

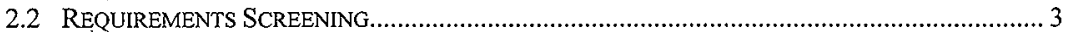

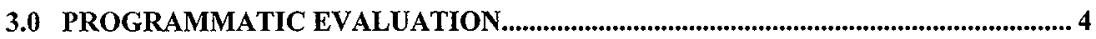

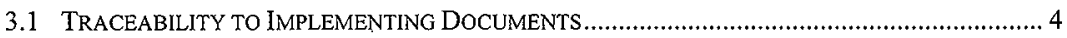

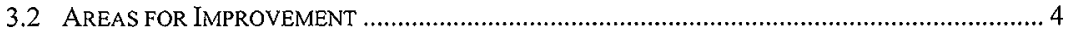

4.0 EQUIPMENT EVALUATION................................................................................................. 13

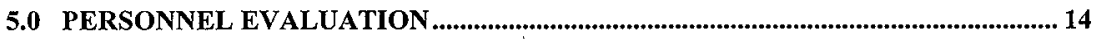

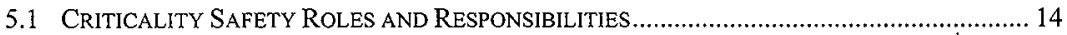

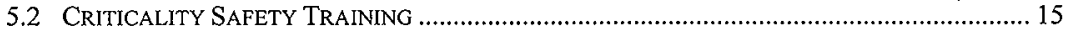

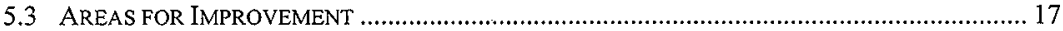

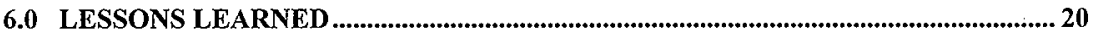

7.0 CONCLUSIONS AND RECOMMENDATIONS........................................................... 24

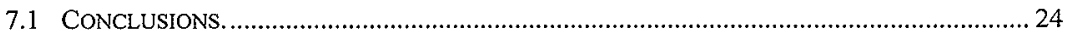

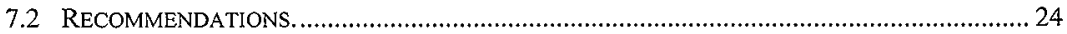

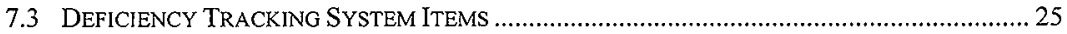

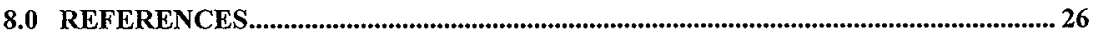

\section{APPENDICES}

APPENDIX A Review Plan for the Criticality Safety Program at the Tank Waste Remediation System (TWRS)

APPENDIX B Matrix of TWRS-Specific Requirements to Implementing Documents APPENDIX C Matrix of Hanford Site (HNF-PRO) Requirements to TWRS Implementing Documents 
TWR-3721 Rev. 0

This page intentionally left blank 


\subsection{INTRODUCTION}

\subsection{Purpose}

The purpose of this document is to provide the results of an internal management review of the Tank Waste Remediation System criticality safety program, performed in advance of the $\mathrm{DOE} / \mathrm{RL}$ assessment for closure of the Nuclear Criticality Safety Issue. This review comprehensively assesses the viability of the administrative program, the final requirement for closure of the safety issue, by evaluating whether the program meets the criticality safety requirements defined in the following documents.

WHC-SD-MP-SRID-001, High Level Waste Storage Tank Farms/242-A Evaporator Standards/Requirements Identification Document

HNF-SD-WM-TSR-006, Tank Waste Remediation System Technical Safety Requirements Project Hanford Management System procedures (HNF-PRO documents)

This review includes the requirements of American National Standards Institute/American Nuclear Society (ANSI/ANS)-8.19, Administrative Practices for Nuclear Criticality Safety, and other related ANSI/ANS-8 series standards as required under DOE Order 5480.24 .

\subsection{Background}

The Tank Waste Remediation System (TWRS) criticality safety program applies to activities associated with the safe receipt, handling, and storage of waste in TWRS facilities. Specifically, the program applies to the following fissionable materials storage areas:

- High-level waste (HLW) storage tanks and ancillary equipment,

- Double-contained receiver tanks (DCRTs) and ancillary equipment,

- Miscellaneous inactive storage facilities (MISFs) and other inactive facilities,

- Ponds, cribs, and ditches.

The original HLW tank design (149 tanks constructed between 1943 and 1964) was a single, reinforced concrete shell and dome with mild carbon steel lining the bottom and walls. The capacity of these single-shell tanks (SST) varies from 208,000 to 3.79 million liters $(55,000$ to 1 million gallons). Twenty-eight (28) double-shell tanks (DST) were built between 1968 and 1986. The DST design is a carbon-steel primary tank and a carbon-steel secondary tank in a protective reinforced-concrete shell with a capacity of 3.79 million liters (1 million gallons). The ancillary systems that support operations in the tank farms include underground and aboveground waste transfer pipelines, valves, diversion boxes, jumper connectors, waste transfer pumps, catch tanks, process pits, the 204-AR Waste Unloading Facility, and the 244-AR vault. The DCRTs are used for temporary storage of waste during transfer operations. Inactive facilities (including MISFs), and ponds, cribs, and ditches may contain fissionable material and are therefore addressed by the criticality safety program.

The HLW tanks are used to store wastes generated by Hanford Site production operations involved in the chemical processing of irradiated uranium. The wastes discharged to the tanks were alkaline slurries containing heavy metals; organic solvents, inorganic salts, uranium, 
plutonium, and mixed fission products. The current TWRS mission is to continue to safely store the remaining tank waste and to conduct waste retrieval and disposal activities in support of the long-term goal for the complete removal and final disposition of the tank waste. Activities are performed at TWRS in a manner that complies with environmental regulations and minimizes public and worker risk.

A series of nuclear criticality safety reviews of the HLW tanks, conducted in 1991 and 1992 , led to the conclusion that an insufficient technical basis existed to support the claim that accidental criticality was not credible in the tanks, and the subsequent declaration of an Unreviewed Safety Question (USQ). After extensive study of the available process data, waste chemistry data, and characterization sample data, it was determined that the waste in the tanks was subcritical by a large margin. Closure of the USQ was approved in March 1994 (Grumbly 1994). At that time, a safety issue was defined related to nuclear criticality safety in the HLW tanks. The safety issue comprised a technical part and an administrative part. The technical portion of the safety issue addressed the uncertainty in the amounts and distribution of fissile material in the HIW tanks. The administrative part related to the need to strengthen the nuclear criticality safety program. Resolution of the safety issue was identified as Hanford Federal Facility Agreement and Consent Order (Tri-Party Agreement) Milestone M-40-12, with a due date of September 1999 (Ecology et al. 1996).

Collectively, the DSTs and SSTs contain an estimated 500 to $1,000 \mathrm{~kg}(1,100$ to $2,200 \mathrm{lb})$ of plutonium. Analyses of tank waste samples show that the plutonium content of the waste is primarily associated with the sludge phase. WHC-SD-WM-TI-725, Tank Farm Nuclear Criticality Review, established that fissile material in the waste tanks is distributed at subcritical concentrations and that no mechanisms exist to concentrate the fissile material to result in an accidental nuclear criticality. The technical portion of the TWRS Nuclear Criticality Safety Issue was resolved (i.e., is considered closed) (Tayloe 1996) on the basis of the arguments in WHC-SD-WM-TI-725. However, formal closure of the safety issue remains contingent on establishing that the administrative criticality safety program at TWRS is sufficient to ensure continued safe operations in support of the TWRS Mission.

A technical evaluation of the phenomenological aspects of the receipt and storage of waste at TWRS led to the conclusion, documented in HNF-SD-WM-BIO-001, Tank Waste Remediation System Basis for Interim Operation Section 5.3.2.1, that a criticality accident at TWRS is "beyond extremely unlikely." The TWRS criticality safety program, therefore, consists primarily of administrative controls designed to protect against altering the waste inventory in a way that could increase the probability of a criticality accident.

\subsection{Review Scope}

This review supports closure of the administrative portion of the TWRS Nuclear Criticality Safety Issue by evaluating the TWRS nuclear criticality safety program for compliance with the applicable requirements (see Section 2.0 of this document). The review plan and identification of the team members is included in Appendix A. 
TWR-3721 Rev. 0

\subsection{REVIEW REQUIREMENTS}

\subsection{Requirements Identification}

Nuclear criticality safety requirements are identified at the Tank Waste Remediation System (TWRS) from several sources. The primary requirements sources include WHC-SD-MP-SRID-001, High Level Waste Storage Tank Farms/242-A Evaporator Standards/Requirements Identification Document (the S/RID), HNF-SD-WM-TSR-006, Tank Waste Remediation System Technical Safety Requirements (the TSR), and Project Hanford Management System procedures (HNF-PRO documents). The requirements were taken from the source documents that were current in July through September 1998.

\subsection{Requirements Screening}

2.2.1 Applicability of Nuclear Criticality Safety Requirements. The requirements from the above sources were reviewed to identify those that apply to the nuclear criticality safety program at TWRS.

2.2.2 Review Criteria. To facilitate the review of implementation of requirements, the requirements found to be applicable to the nuclear criticality safety program at TWRS were binned into the following three groupings:

(1) Program Requirements

(2) Equipment Requirements

(3) Personnel Requirements

Detailed assessments were performed for each of these three groupings. The groupings facilitate both the review, and the development of actions to address areas for improvement identified during the review. 
TWR-3721 Rev. 0

\subsection{PROGRAMMATIC EVALUATION}

\subsection{Traceability to Implementing Documents}

The requirements found to be applicable to the nuclear criticality safety program at TWRS were then mapped to implementing documents. A matrix of TWRS-specific (S/RID and TSR) requirements to implementing documents is provided in Appendix B. That matrix also includes the American National Standards Institute (ANSI)/American Nuclear Society (ANS) standards required by reference in DOE 5480.24. A matrix of Project Hanford Management System (HNF-PRO) requirements to implementing documents is provided in Appendix C.

\subsection{Items for Improvement}

Table 3-1 provides a summary of items for improvement that were identified from the matrices described above, and from a review of HNF-IP-1266, Tank Farms Operations Administrative Controls, Chapter 5.7, "Nuclear Criticality Safety," against the TWRS Authorization Basis. The table also identifies recommended actions to address each item. In addition to the description of each recommended action, the table includes a column to indicate whether the action includes revising HNF-IP-1266, Tank Farms Operations Administrative Controls (with an entry of Yes, No, or Maybe), and a column that briefly identifies other documents that may require change. 


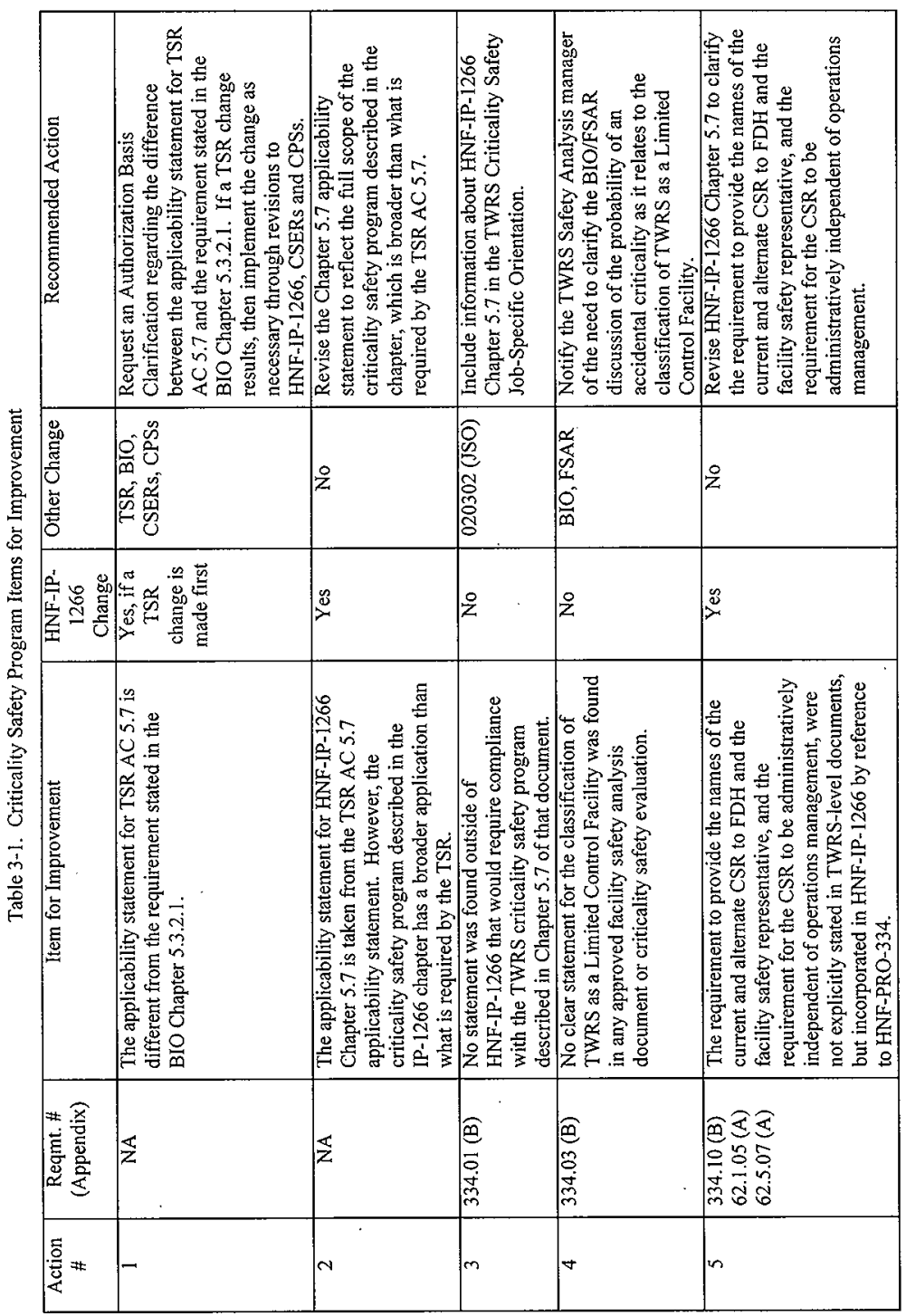


TWR-3721 Rev. 0

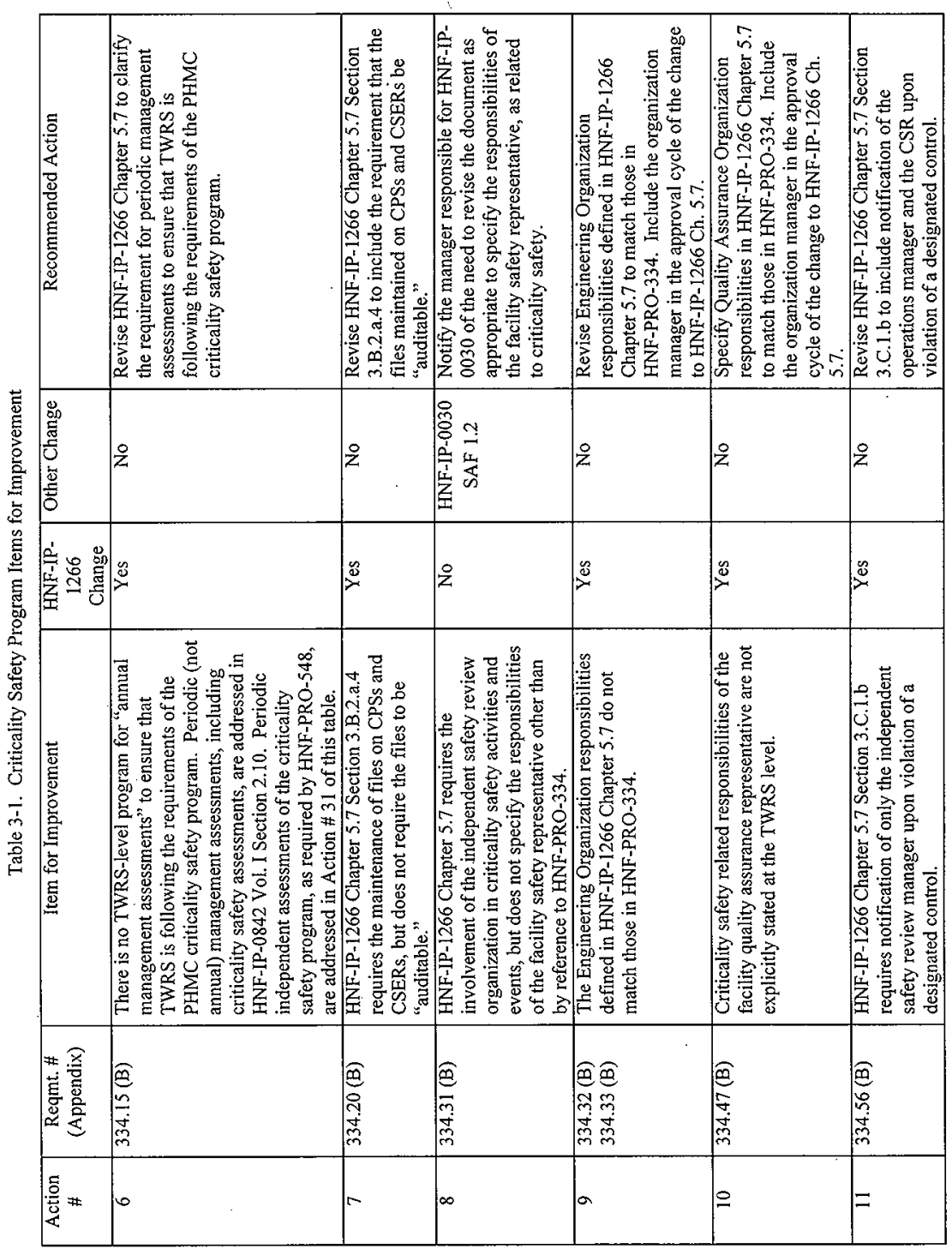


TWR-3721 Rev. 0

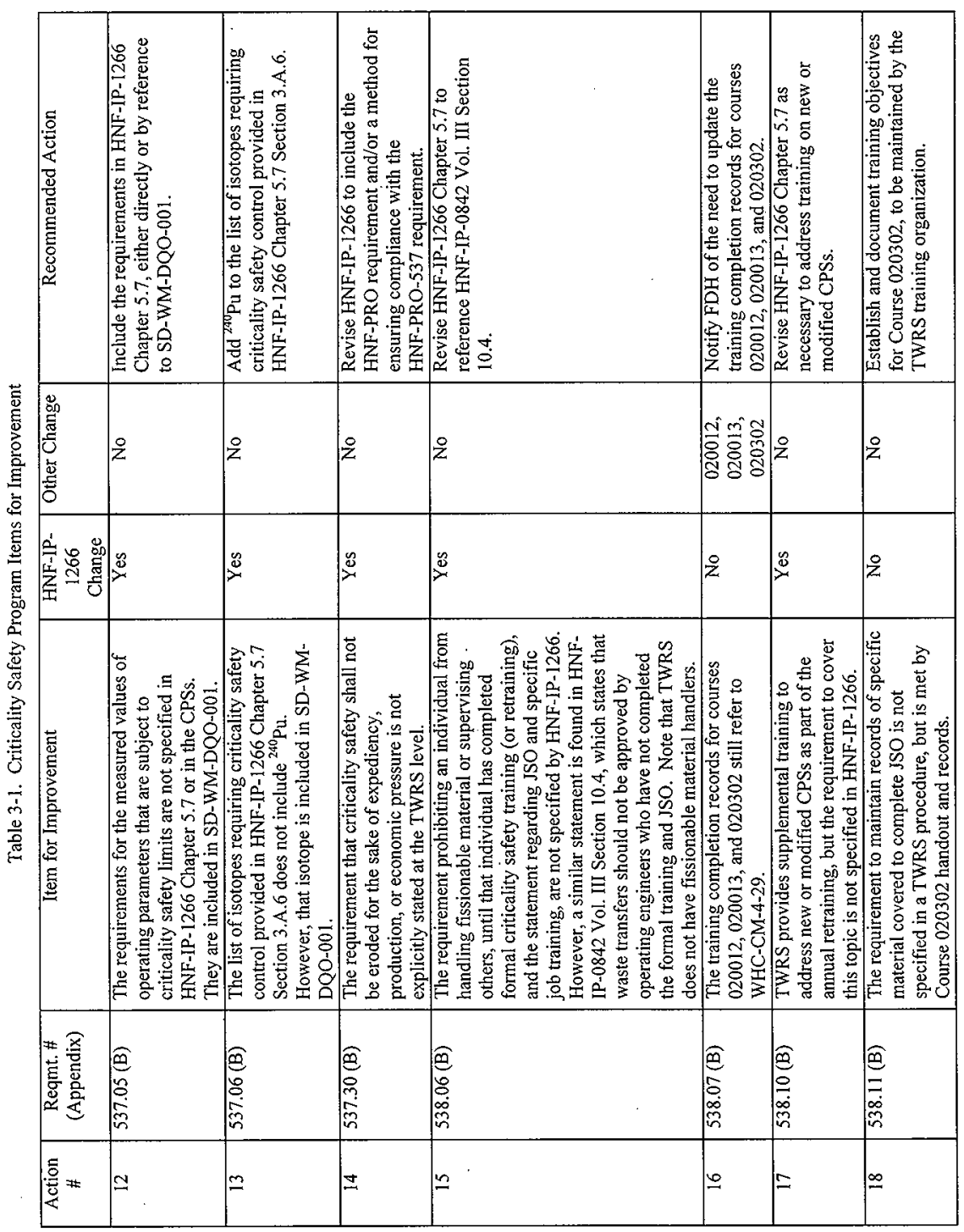


TWR-3721' Rev. 0

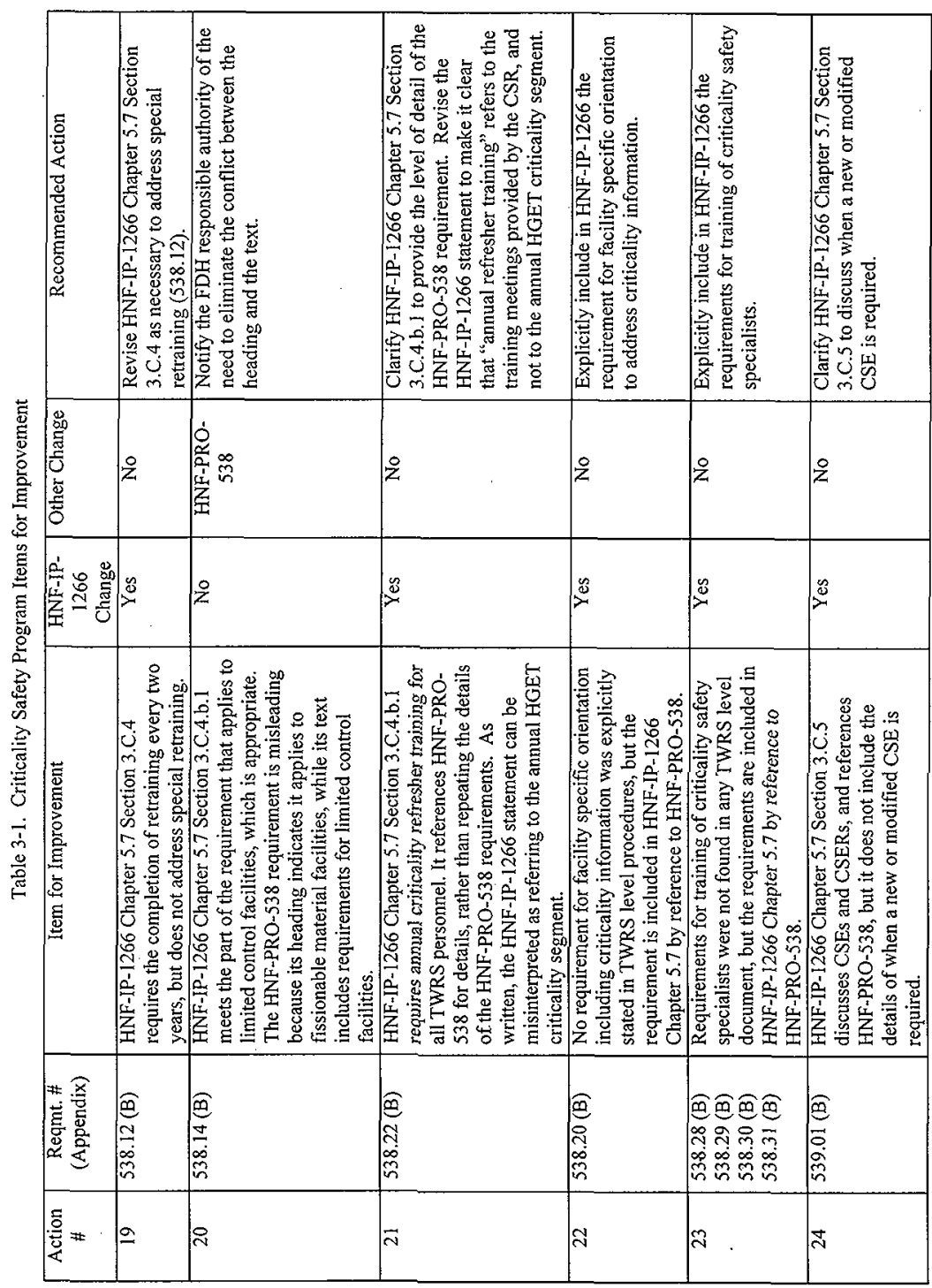




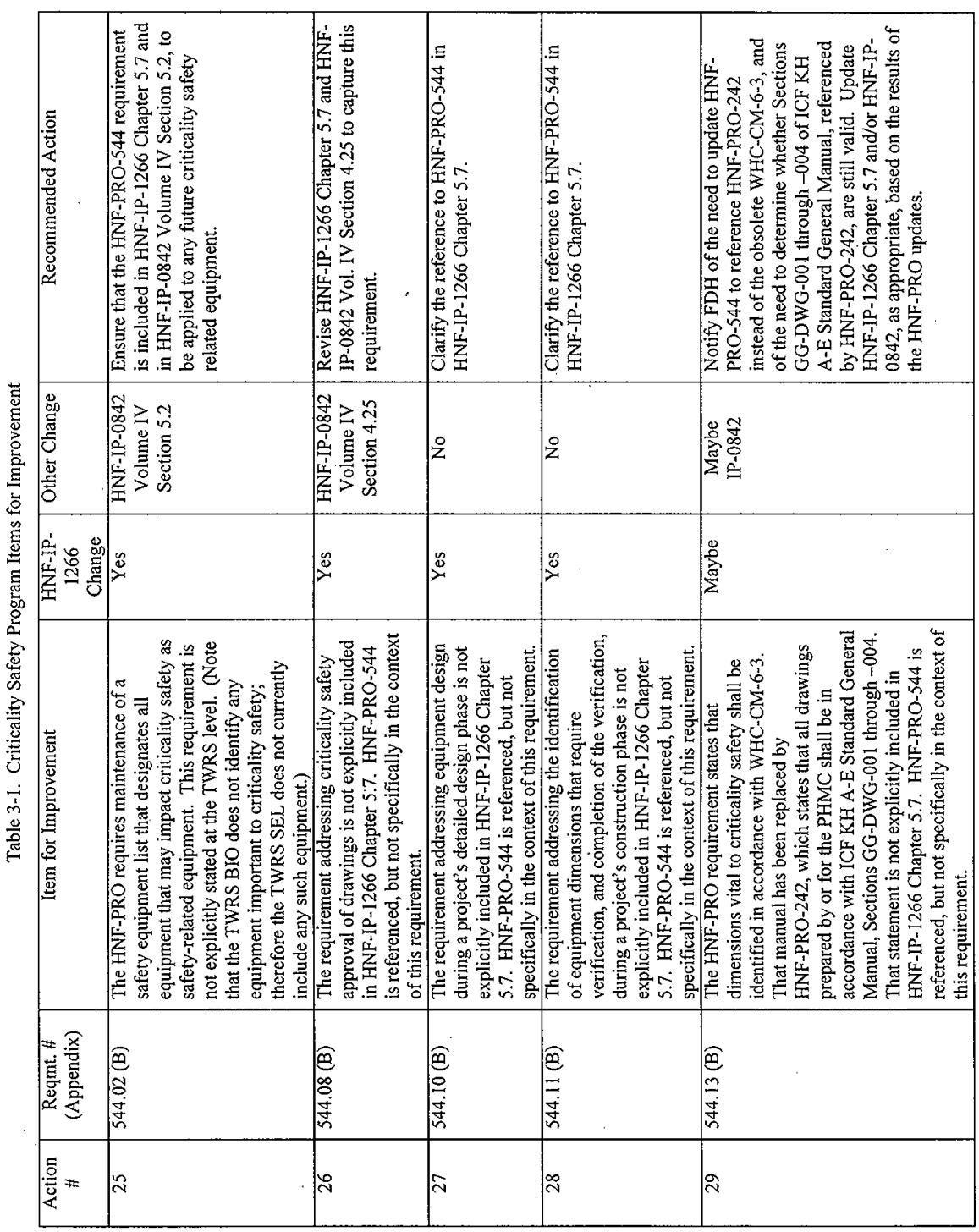




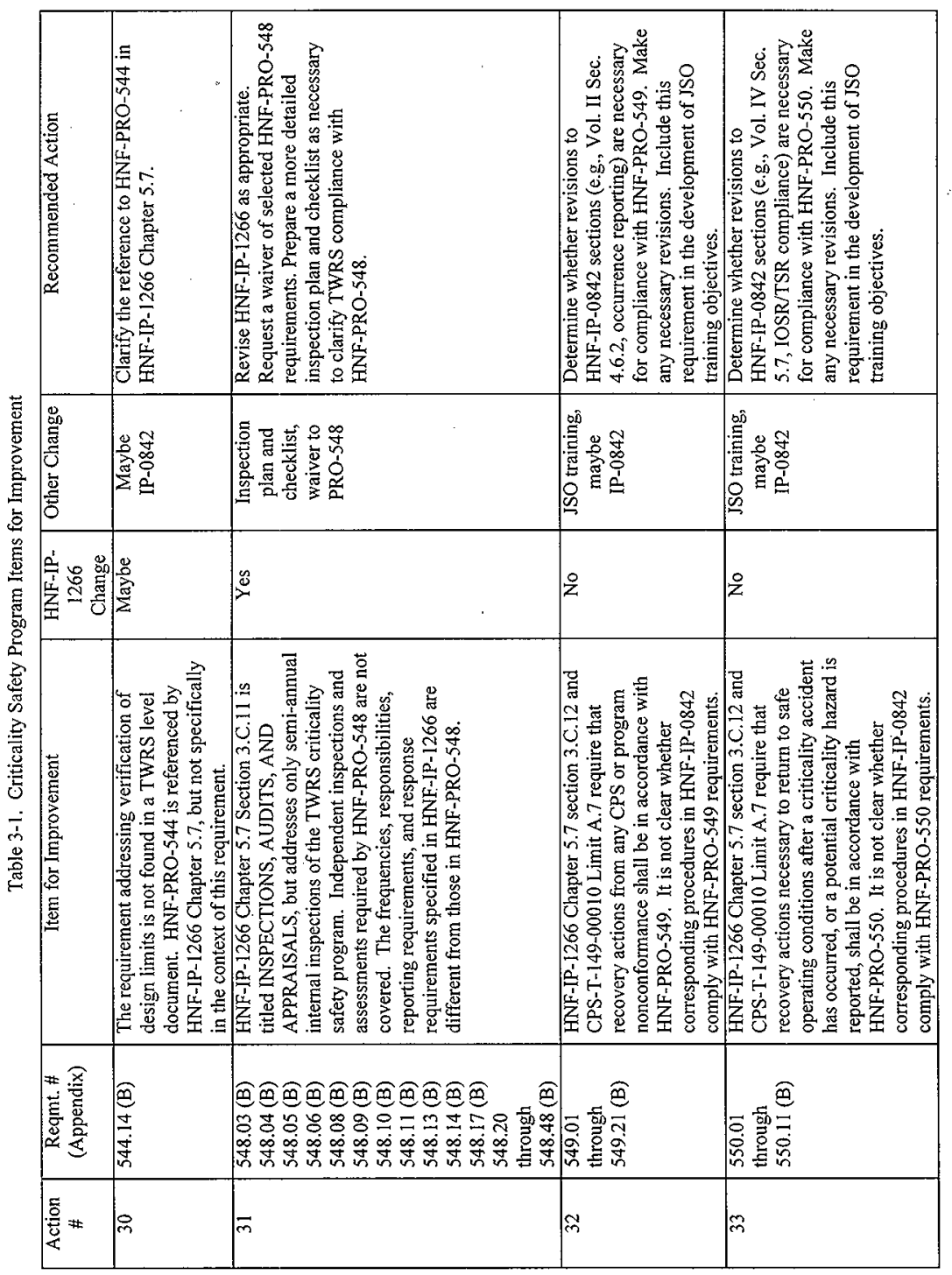




\begin{tabular}{|c|c|c|c|c|c|c|c|}
\hline 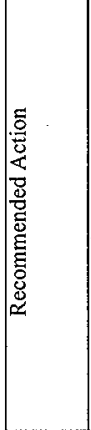 & 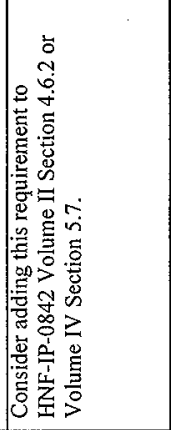 & 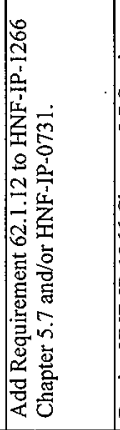 & 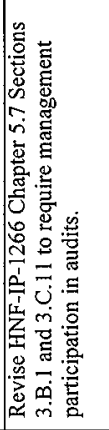 & 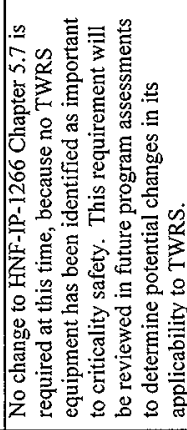 & 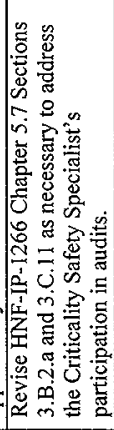 & 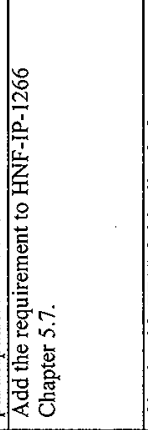 & 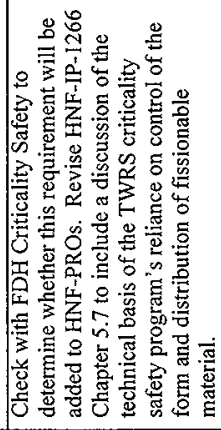 \\
\hline 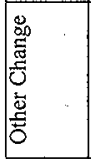 & 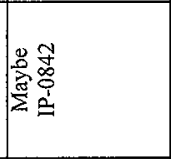 & 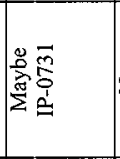 & z & ż & 울 & Z & $\ddot{z}$ \\
\hline 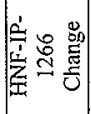 & & 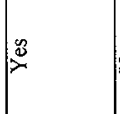 & 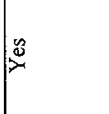 & i̊ & 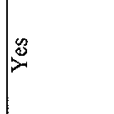 & $\sum_{>}^{\infty}$ & $y_{2}^{2}$ \\
\hline 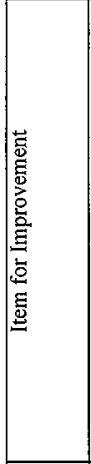 & 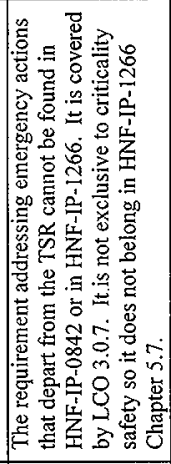 & 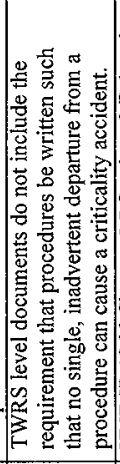 & 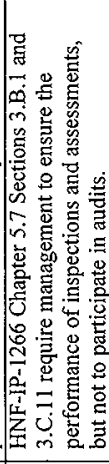 & 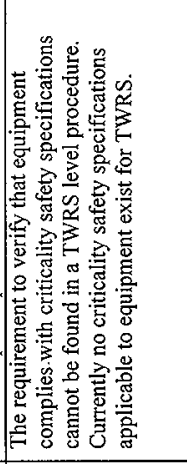 & 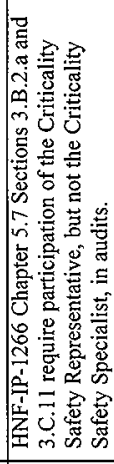 & 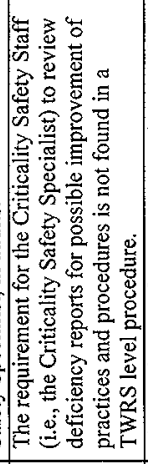 & 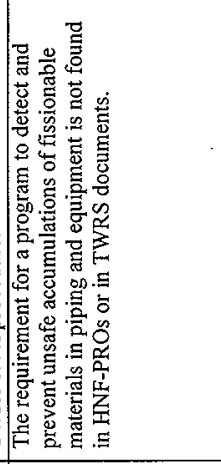 \\
\hline 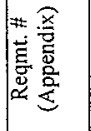 & $\frac{5}{6}$ & 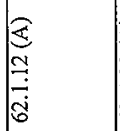 & $\begin{array}{l}3 \\
\vdots \\
8 \\
0 \\
0 \\
\hat{i} \\
0\end{array}$ & 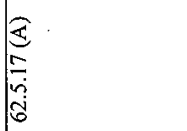 & 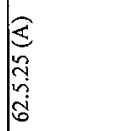 & 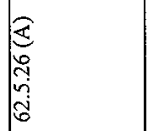 & $\begin{array}{l}8 \\
6 \\
6\end{array}$ \\
\hline 总 & 荡 & $m$ & in & $\approx$ & $\infty$ & min & i \\
\hline
\end{tabular}




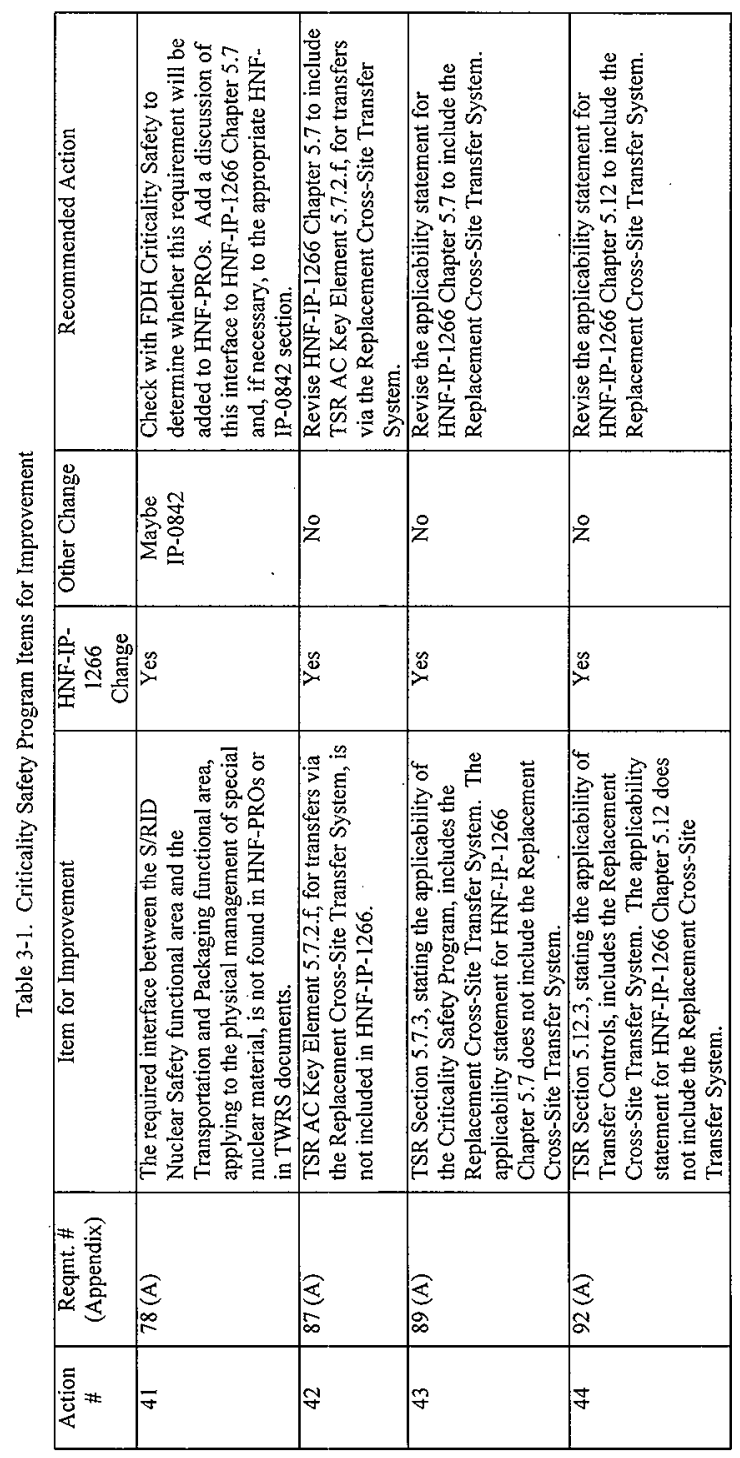




\subsection{EQUIPMENT EVALUATION}

Requirements applicable to equipment are addressed in Section 3.0, Programmatic Evaluation. However, neither the analysis summarized in Section 5.3.2.1 of HNF-SD-WM-BIO-001, Tank Waste Remediation System Basis for Interim Operation (BIO), nor HNF-SD-WM-SEL-040, TWRS Facility Safety Equipment List, prepared in conjunction with the BIO, identifies any structures, systems, or components for prevention or mitigation of a criticality accident. At this time there are no TWRS equipment items to evaluate for compliance with the criticality safety requirements. 
TWR-3721 Rev. 0

\subsection{PERSONNEL EVALUATION}

\subsection{Criticality Safety Roles and Responsibilities}

Figure 5-1 shows the relationships between the various Lockheed Martin Hanford Corporation (LMHC) organizations that have responsibilities for aspects of TWRS nuclear criticality safety. Following is a summary of the responsibilities of each organization shown in the figure.

LMHC President and General Manager: Overall responsibility for safe operations at TWRS

Tank Waste Operations: Responsible for TWRS Operations

Shift Operations: Responsible for qualification and training of Shift Managers/Operations Engineers

Tank Farms Facility Operations: Responsible for managing/approving Tank Farm transfer activities

TWRS Training: Responsible for maintaining training and qualification records for TWRS, and for serving as training interface with FDH

Environment, Safety, Health and Quality Assurance: Responsible for ESH\&QA at TWRS

Safety Services: Responsible for providing independent safety reviews of documents and activities, and for qualification and training of Safety Engineers

Quality Assurance: Responsible for providing quality assurance reviews of documents and activities, and for qualification and training of Quality Engineers

TWRS Technical Operations and Engineering (TOE): Responsible for engineering activities at TWRS

Chief Engineer: Responsible for final approval of qualifications for cognizant engineers and design authorities

Process Engineering: Responsible for maintenance of the plutonium inventory database, Data Quality Objectives, and waste compatibility program

Plant Engineering: Responsible for coordinating reviews of new and modified processes, systems, and equipment, and for training and qualification of cognizant engineers and design authorities

Nuclear Safety and Licensing: Responsible for the TWRS criticality safety program

Nuclear Safety Support: Direct line management for the TWRS criticality safety representative and criticality safety specialist/engineer/analyst; responsible for implementation of the criticality safety program and for providing criticality safety subject matter experts 


\subsection{Criticality Safety Training}

Personnel training and qualification requirements are addressed in Section 3.0, Programmatic Evaluation. For determination of Personnel Readiness, records were reviewed to ensure that individuals who are required to receive criticality safety training or to meet other qualification requirements actually do meet those requirements.

Review of the requirements for nuclear criticality safety training of TWRS personnel, as found in HNF-IP-1266, Tank Farms Operations Administrative Controls, and HNF-PRO-538, Criticality Safety Training, led to the identification of five groups of personnel:

all TWRS personnel, exempt personnel, engineering personnel, the Criticality Safety Representative (CSR), and assistants to the CSR.

\subsubsection{All TWRS Personnel as stated in HNF-IP-1266, Section 5.7.3.C.4.b.2}

Requirement: minimum criticality safety training with annual Hanford General Employee Training (HGET) and annual refresher training

TWRS status: satisfied by HGET and by annual CSR presentations at shift meetings

\subsubsection{Exempt personnel}

Requirement: Training designated by facility manager as appropriate for specific job assignment (HNF-IP-1266, Section 5.7.3.C.4.b.2), managers and engineers criticality safety training course (initial training 020012 , retrain 020013 , job specific orientation 020302 is usually included). HNF-PRO-538 (included by reference in HNF-IP-1266, Section 5.7.3.C.4) requires training for managers and supervisors who oversee the activities of fissionable material handlers.

TWRS status: TWRS has no personnel designated as fissionable material handlers (HNF-SD-WM-HSP-002). Tank Waste Operations has responsibility for personnel involved in activities that could alter the form or distribution of fissionable material (principally waste transfers). HNF-IP-0842, Vol. III, Section 10.4, "Operations Engineer/Shift Operations Manager Training" assigns nuclear criticality safety classroom training to operations engineers/shift operations managers and prohibits operating engineers who have not completed the training from approving waste tank transfers.

\subsubsection{Engineering personnel}

Requirement: Training designated by engineering managers as appropriate for specific job assignment (HNF-IP-1266, Section 5.7.3.C.4.b.3), managers and engineers criticality safety training course (initial training 020012 , retrain 
020013, job specific orientation 020302 is usually included). HNF-PRO 538 (included by reference in HNF-1P-1266, Section 5.7.3.C.4) provides the following additional detail relative to engineering personnel requiring training:

managers and engineers directly involved in the design of equipment for the handling, storage or transportation of significant quantities of fissionable material

safety and QA managers or engineers who directly oversee or audit criticality safety programs

TWRS status: TWRS Technical Operations and Engineering has responsibility for cognizant engineers and design authority. HNF-IP-0842, Volume III, Section 10.3, "Technical Staff Qualification Program Description" requires Qualification Cards for Cognizant Engineers (350860), Quality Assurance Engineers (350885) and Design Authority (350865). Management directly responsible for the individual and the Chief Engineer are responsible for approving qualifications for Cognizant Engineers and Design Authority. Currently, every cognizant engineer and design authority, with three exceptions, is current with nuclear criticality safety training. The responsibilities of the three exceptions have been reviewed, and it has been verified that they do not require criticality safety training.

Line managers and the manager of TWRS Quality Assurance are responsible for approval of qualifications for Quality Assurance Engineers. No QA managers or engineers are currently trained. However, the QA representative who performed the most recent audit of criticality safety was matrixed from the safety organization, and was verified to be current in TWRS criticality safety training. All other members of the assessment team were external employees qualified by resume.

The requirements, responsibilities, and instructions for qualification of safety professionals are defined in WHC-IP-0030, Safety Department Administrative Manual, SAF-1.2 Rev. 4, "Safety Personnel Qualification Procedure." The Core Training Plan described in section 4.4 of that procedure cites Criticality Safety Training as optional but recommended for Nuclear Safety Engineers. This requirement applies to safety engineers within TWRS Environmental, Safety and Quality Assurance who are responsible for the Safety reviews as required in HNF-PRO-233, Review and Approval of Documents. The individual performing safety reviews was verified to be current in criticality safety training.

TWRS Nuclear Safety and Licensing (NS\&L) is responsible for direct oversight of the TWRS Criticality Safety Program. Within NS\&L, workscope, budget and personnel responsible for overseeing the nuclear criticality safety program are part of the Nuclear Safety Support group. 
The criticality safety representative, the criticality safety specialist, and the manager directly responsible for the criticality safety program were verified to be current in criticality safety training.

5.2.4 Criticality Safety Representative (CSR) as stated in HNF-IP-1266, Section 5.7.3.C.4.a.3 through 5.7.3.C.4.a.6

Requirement: managers and engineers criticality safety training course (initial training 020012, retrain 020013)

facility specific orientation (JSO training 020302)

examination by qualified board

annual training meeting

TWRS status: Training is specified in HNF-IP-1266 as stated above and a Qualification Card and Guide for TWRS Facility Criticality Safety Engineer (350004) has been developed and is being incorporated into the CSR required training. The CSR's training and qualifications have been verified to be valid until May 1999, when re-examination by the qualified board is required.

5.2.5 CSR assistants as stated in HNF-IP-1266, Section 5.7.3.C.4.a.3

Requirement: managers and engineers criticality safety training course (initial training 020012 , retrain 020013 , job specific orientation 020302 is usually included)

TWRS status: Activities supporting the nuclear criticality safety program are the responsibility of the Nuclear Safety Support group. Work assignments within NS\&L are made using Individual Work Authorizations (IWAs). IWAs for staff outside of NSS yet still within NS\&L require a one-over signature thereby notifying responsible management that assignments have been made related to nuclear criticality safety and appropriate training should be assigned as necessary. Personnel external to NS\&L are tasked via the Task Package Commitment Agreement (TPCA) process. That process requires line management concurrence/acceptance and therefore notification of job responsibilities directly related to nuclear criticality safety. All personnel with criticality safety related responsibilities assigned through the TPCA process have been verified to be current in criticality safety training.

\subsection{Areas for Improvement}

Management awareness of nuclear criticality safety training requirements should be reinforced because of the major reorganizations within TWRS since the beginning of fiscal year 1999 . 
TWR-3721 Rev. 0

The training requirements specified in HNF-IP-1266 Chapter 5.7 should be updated and clarified consistent with HNF-PRO-538, particularly the requirement for annual refresher training. 


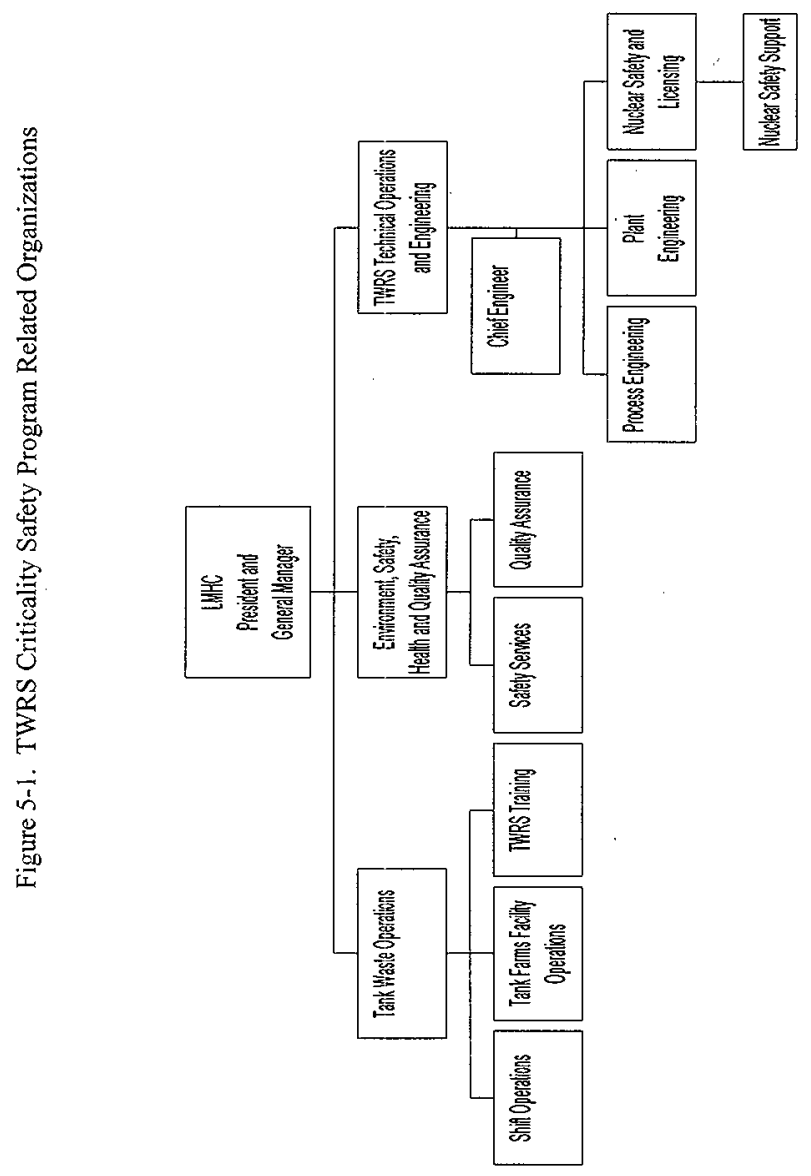




\subsection{LESSONS LEARNED}

Criticality safety program assessment reports that were prepared for DOE facilities other than TWRS were reviewed for items that could also be applied as "Lessons Learned" at TWRS. Those items, and the TWRS response to each, are listed below.

The following recommendations and suggestion were extracted from DOE/EH-0571, The Plutonium Finishing Plant Criticality Safety Program Review.

Recommendation 3. In the absence of effective criticality safety programs at $D O E-R L$ and FDH, consideration should be given to extending the exclusivity clause for $F D N W$ because, even with the above programmatic deficiencies noted, several members of the FDNW criticality safety staff have Hanford and PFP experience and the demonstrated technical ability to provide support to PFP. DOE-RL, $F D H$, and BWHC should form a partnership to ensure that FDNW provides the best criticality safety engineer (CSE) support available to PFP in the near term until specific guidance is developed by FDH to provide necessary nuclear criticality safety (NCS) technical support in the longer term. Alternatively, $B W H C$ could retain its own CSE staff as permanent employees or FDH could assume the NCS role for the site and matrix CSE to the facilities as needed. While the first of these three options fits best with the current Integrating Management Contractor arrangements, the third has the best chance of providing a vigorous NCS program for Hanford.

TWRS Response: TWRS retains its own criticality safety engineering staff as permanent employees within the Nuclear Safety Support organization.

Recommendation 11. FDH and FDNW should review the Rocky Flats criticality safety evaluation procedure for documenting controls and contingencies.

TWRS Response: The Savannah River criticality safety program will be reviewed, rather than the Rocky Flats program. The original recommendation applies to the Plutonium Finishing Plant, which is similar to the Rocky Flats facility. Because the TWRS facility is similar to Savannah River, it is expected that aspects of the Savannah River program would be more applicable to TWRS.

Recommendation 13. The FDH CSE training and qualification program for PFP should include formal coursework (similar to that at $Y-12$ and Savannah River), on-thejob training under appropriate NCS mentors, and a formal program of familiarization with facility operations followed by an oral board similar to that used to qualify CSRs. The qualification for peer reviewers should require specific facility related NCS experience and evidence of technical competence and leadership such as technical papers and reports at professional conferences. FDH should ensure funding is provided for CSE training and qualification.

TWRS Response: Criticality safety classroom training is provided by a private contractor. Under the terms of the contract, the FDH Training 
Programs/Standards organization is responsible for monitoring the course 4 times per year, qualifying instructors, and establishing performance measures. Changes in course content/curriculum are reviewed and approved by the FDH Nuclear Criticality Safety Technical Authority, who solicits feedback from facility CSRs.

In addition, facility orientation requirements and other relevant aspects of the above recommendation have been included in the TWRS Criticality Safety Engineer Qualification Card, required for completion by the CSR and the CSE.

Recommendation 18. All BWHC, FDH, and FDNW criticality safety personnel working at or for PFP should have professional development opportunities related to ANSLIANS-8 standards and other subjects pertaining to their areas of support for PFP work.

TWRS Response: Professional development activities for criticality safety personnel are included in the FY 1999 plan and budget for TWRS Criticality Safety through the Technical Basis Review process.

Recommendation 22. Some of the NCS responsibilities currently assigned to the CSR should be transferred to the FDNW CSE supporting PFP. Amonig these responsibilities are reviewing operating procedures and postings, process and equipment modifications, assisting with NCS training, performing regular audits, and evaluating infractions and developing corrective actions.

TWRS Response. The TWRS CSR and CSE positions are part of the same organization, providing synergy and opportunities for cross training to increase the skills of both. There are no procedural barriers to utilizing the CSE for performing audits, evaluating infractions, or developing corrective actions.

Suggestion 8. Implementation of the Deficiency Tracking System should be improved. Emphasis should be focused on training and other guidance to ensure that project managers use the DTS as a management system and tool for improving performance rather than allowing it to become an administrative "bean counting" device.

TWRS Response. TWRS requires use of the Deficiency Tracking System as a management tool by including the DTS as an integral part of its Management Assessment Program and Corrective Action Management process. Reference HNF-IP-0842, TWRS Administration, Volume I, Section 2.4 "Corrective Action Management," and Section 2.10 "Management Assessment Program." 
TWR-3721 Rev. 0

The following recommendations were extracted from First-Quarter Criticality Safety Program Review at the Oak Ridge Y-12 Plant, dated September 1998.

Recommendation 1. Identify subject matter experts (SMEs) that have personally operated processes, have a thorough understanding of the system/process, are capable of identifying credible process upsets important to criticality safety, and who are authorized to work with the Nuclear Criticality Safety Organization (NCSO) to develop and accept criticality controls on behalf of supervision. Require SMEs and Technical Support personnel to team with the NCS engineers in developing criticality safety evaluations (CSEs) to describe the process, develop assumptions, and identify contingencies and appropriate controls that are understood and accepted by Operations. Operations should formally accept CSEs by signing them. Make the CSEs or appropriate sections from them available to line supervision. Rocky Flats provides sections 1-9) (See DOE-STD-3007-93) to operations.

TWRS Response. PHMC procedure HNF-PRO-334, Criticality Safety: General Requirements, is being revised to specifically address the responsibilities of Operations supervision and the criticality safety specialist. These responsibilities under the revised procedure include the following.

Operations Supervision. Review contingency conditions presented in new or revised criticality safety evaluation reports (CSERs) to ensure that all credible accidents, errors, or equipment malfunctions are considered. Develop or participate in the development of written procedures applicable to the operations under their control. Maintain operating procedures to reflect changes in operations. Verify compliance with new or modified criticality prevention specifications (CPSs), including those for new equipment before its use.

Criticality Safety Specialist. Provide technical guidance for the preparation, development, and technical peer review of all new CSERs, and revisions to previously prepared CSERs. Provide technical guidance for the preparation, development, and review of new and revised CPSs at Fissionable Material Facilities and Limited Control Facilities. The criticality safety specialist's involvement in CPSs at Isolated Facilities is determined by the CSR and facility management on an as-needed basis.

Recommendation 5. The Team recommends that the NCSO CSE development process be restructured to ensure that stand-alone CSEs are developed that meet the intent of ANSU/ANS-8.19 Section 8. To that end, the Team recommends the following specific steps be taken. (Followed by six steps not included here.)

TWRS Response. The development of CSERs at TWRS is governed by PHMC procedure HNF-PRO-539, Criticality Safety Evaluations, and by HNF-IP-1266 Chapter 5.7, "Nuclear Criticality Safety," section 5.7.3.C.5. 
TWR-3721 Rev. 0

Both procedures have been verified to be compliant with the requirements of ANSI/ANS-8.19 Section 8. See Table A-1, Requirements 62.5.36 through 62.5.39, in Appendix B of this document. 
TWR-3721 Rev. 0

\subsection{CONCLUSIONS AND RECOMMENDATIONS}

\subsection{Conclusions}

The evaluations of program, personnel, and equipment readiness related to nuclear criticality safety did not identify any issues of noncompliance. However, the evaluations led to the identification of areas for improvement, which are described below for each of the three areas of the assessment. This review also satisfied the objectives of the Phase II verification of the criticality safety requirements of the S/RID.

\subsection{Recommendations}

7.2.1 Areas for Improvement from the Programmatic Evaluation. These recommended actions are identified in Table $3-1$, and summarized here.

Revise PHMC internal publications as necessary to:

formalize aspects of the nuclear criticality safety program not currently documented,

provide clarification and eliminate ambiguities, and

update the program requirements for conformance with the $\mathrm{PHMC}$ procedures (HNF-PRO documents) that replaced WHC-CM-4-29, Nuclear Criticality Safety Manual.

The specific internal publications, owned by TWRS, that require revisions are:

HNF-IP-1266, Tank Farms Operations Administrative Controls, Chapter 5.7, "Nuclear Criticality Safety," and Chapter 5.12, "Transfer Controls"

HNF-IP-0842, TWRS Administration

HNF-IP-0731, Tank Waste Remediation Systems Technical Procedure Format and Preparation Guide

Request waivers or revisions to specific requirements from the following HNF-PRO documents, where the requirements need improvement or are not appropriate for implementation at TWRS.

HNF-PRO-334, Criticality Safety General Requirements

HNF-PRO-537, Criticality Safety Control of Fissionable Material

HNF-PRO-538, Criticality Safety Training

HNF-PRO-548, Criticality Safety Inspections and Assessments

Update the content of the Criticality Safety Job-Specific Orientation (Course Number 020302) as necessary to reflect current nuclear criticality safety program requirements, and to incorporate program improvements that result from this assessment. Notify the manager responsible for PHMC level criticality training courses of recommended improvements to those courses.

Prepare and process changes to the TWRS Authorization Basis, criticality safety evaluation reports, and/or criticality prevention specifications as detailed in Table 3-1.

7.2.2 Areas for Improvement from the Equipment Evaluation. TWRS has no structures, systems, or components credited with prevention or mitigation of a criticality accident, as stated 
in Section 4.0 of this document. Therefore, at this time, no criticality safety requirements apply to any equipment at TWRS, and no areas for improvement have been identified.

7.2.3 Areas for Improvement from the Personnel Evaluation. The following actions are recommended in addition to the training-related changes to PHMC internal publications and training courses addressed in Section 7.2.1.

Reinforce management awareness of nuclear criticality safety training requirements. This is necessary because of major reorganizations within TWRS that have occurred since the beginning of fiscal year 1999.

Ensure that personnel who are not current in criticality safety training as required for their job positions (e.g., Quality Assurance managers and engineers) are aware of training requirements. Those personnel shall not perform or authorize criticality safety related activities until they complete the training.

Revise HNF-IP-1266 Chapter 5.7 to specify that both the CSR and the Criticality Safety Engineer/Specialist are required to complete the Qualification Card and Guide for TWRS Facility Criticality Safety Engineer (350004).

Replace the matrixed support personnel with a permanent employee in the TWRS CSR position within the Nuclear Safety Support organization.

\subsection{Deficiency Tracking System Items}

The following items will be entered into the Deficiency Tracking System.

1) Revise HNF-IP-1266 Chapter 5.7 consistent with the detailed recommendations of this report.

2) Develop detailed training objectives for Criticality Safety Job-Specific Orientation (Course Number 020302).

3) Reinforce TWRS management awareness of nuclear criticality safety training requirements.

4) Develop a more detailed criticality safety inspection plan. 
TWR-3721 Rev. 0

\subsection{REFERENCES}

CPS-T-149-00010, 1998, Waste Storage in Double-Shell Tanks and Associated Equipment, Rev./Mod. I-0, Lockheed Martin Hanford Corporation, Richland, Washington.

DOE/EH 1998, First-Quarter Criticality Safety Program Review at the Oak Ridge Y-12 Plant, U.S. Department of Energy, Office of Environment, Safety and Health, September 1998.

DOE/EH-0571, 1998, The Plutonium Finishing Plant Criticality Safety Program Review. U.S. Department of Energy, Office of Environment, Safety and Health/Office of Nuclear and Facility Safety, May 1998.

Ecology, EPA, and DOE, 1996, Hanford Federal Facility Agreement and Consent Order, as amended, Washington State Department of Ecology, U.S. Environmental Protection Agency, and U.S. Department of Energy, Olympia, Washington.

Grumbly, T. P., 1994, Approval to Close the Criticality Unreviewed Safety Question at the Hanford High-Level Waste Tank Farms (memorandum to Manager, U.S. Department of Energy, Richland Operations Office, March 17), U.S. Department of Energy, Washington, D.C.

HNF-IP-0731, 1997, Tank Waste Remediation Systems Technical Procedure Format and Preparation Guide, Rev./Mod. 5, Lockheed Martin Hanford Corporation, Richland, Washington.

HNF-IP-0842, TWRS Administration, Lockheed Martin Hanford Corporation, Richland, Washington. Sections as listed below.

Volume I, Section 2.4, "Corrective Action Management," Rev. 4, May 1998

Volume I, Section 2.10, "Management Assessment Program," Rev. 1a, November 1997

Volume III, Section 10.3, "Technical Staff Qualification Program Description," Rev. 4, December 1997

Volume III, Section 10.4, "Operations Engineer/Shift Operations Manager Training," Rev. 2a, October 1997

HNF-IP-1266, Tank Farms Operations Administrative Controls, Lockheed Martin Hanford Corporation, Richland, Washington. Chapters as listed below.

Chapter 5.7, "Nuclear Criticality Safety," Rev. 1A, July 1998

Chapter 5.12, "Transfer Controls," Rev. 1C, July 1998

HNF-PRO-233, 1997, Review and Approval of Documents, Rev. 0, Fluor Daniel Hanford, Inc., Richland, Washington.

HNF-PRO-242, 1997, Engineering Drawing Requirements, Rev. 1, Fluor Daniel Hanford, Inc., Richland, Washington.

HNF-PRO-334, 1997, Criticality Safety General Requirements, Rev. 0, Fluor Daniel Hanford, Inc., Richland, Washington. 
TWR-3721 Rev. 0

HNF-PRO-537, 1997, Criticality Safety Control of Fissionable Material, Rev. 0, Fluor Daniel Hanford, Inc., Richland, Washington.

HNF-PRO-538, 1997, Criticality Safety Training, Rev. 0, Fluor Daniel Hanford, Inc., Richland, Washington.

HNF-PRO-539, 1997, Criticality Safety Evaluations, Rev. 0, Fluor Daniel Hanford, Inc., Richland, Washington.

HNF-PRO-548, 1998, Criticality Safety Inspections and Assessments, Rev. 1, Fluor Daniel Hanford, Inc., Richland, Washington.

HNF-PRO-549, 1997, Criticality Safety Nonconformance Response, Rev. 0, Fluor Daniel Hanford, Inc., Richland, Washington.

HNF-PRO-550, 1997, Criticality or Potential Criticality Accident, Rev. 0, Fluor Daniel Hanford, Inc., Richland, Washington.

HNF-SD-WM-BIO-001, 1998, Tank Waste Remediation System Basis for Interim Operation, Rev. 1, Fluor Daniel Hanford, Inc., Richland, Washington.

HNF-SD-WM-HSP-002, 1998, Tank Farms Health and Safety Plan, Rev. 3, Lockheed Martin Hanford Corporation, Richland, Washington.

HNF-SD-WM-SEL-040, 1998, TWRS Facility Safety Equipment List, Rev. 1, Lockheed Martin Hanford Corporation, Richland, Washington.

HNF-SD-WM-TSR-006, 1997, Tank Waste Remediation System Technical Safety Requirements, Rev. 0, Fluor Daniel Hanford, Inc., Richland, Washington.

Tayloe, R. W., 1996, Final Report - Review of Hanford High-Level Radioactive Waste Tank Nuclear Criticality Safety Documentation for DOE Richland Operations Office (external letter to Distribution, September 17), Battelle, Columbus, Ohio.

WHC-CM-4-29, 1996, Nuclear Criticality Safety Manual, Rev. 9, Westinghouse Hanford Company, Richland, Washington.

WHC-CM-6-3, 1992, Drafting Standards Manual, Westinghouse Hanford Company, Richland, Washington.

WHC-IP-0030, 1996, Safety Department Administrative Manual, SAF-1.2, "Safety Personnel Qualification Procedure," Rev. 4, Westinghouse Hanford Company, Richland, Washington.

WHC-SD-MP-SRID-001, 1996, High Level Waste Storage Tank Farms/242-A Evaporator Standards/Requirements Identification Document, Rev. 1, Westinghouse Hanford Company, Richland, Washington. 
TWR-3721 Rev. 0

WHC-SD-WM-TI-725, 1996, Tank Farm Nuclear Criticality Review, Rev. 0A, Westinghouse Hanford Company, Richland, Washington. 
TWR-3721 Rev. 0

\section{APPENDIX A}

Review Plan for the Criticality Safety Program at the Tank Waste Remediation System (TWRS) 
TWR-3721 Rev. 0 .

\section{Review Plan for the Criticality Safety Program at the Tank Waste Remediation System (TWRS)}

Responsible Manager:

M. C. Brady Raap

Lead Assessor:

M. A. Smith-Fewell

Team Members:

T. S. Vail

D. R. Bratzel

N. J. Milliken

The assessment scope is defined as:

- Evaluate the following sources for requirements to be satisfied by the Tank Waste Remediation System (TWRS) Criticality Safety Program:

WHC-SD-MP-SRID-001, Functional Area 18, "Nuclear Safety"

The key elements of the Administrative Control programs of HNF-SD-WM-TSR-006

The ANS standards invoked by DOE Order 5480.24, Section 7.a

- Identify which requirements from the above sources are applicable to the TWRS Criticality Safety Program. Provide a justification for those requirements judged to be not applicable.

The assessment performance objectives are:

1. Categorize each requirement according to its applicability to the following areas:

- Program Readiness

- Personnel Readiness

- Hardware Readiness

2. Identify the elements necessary for demonstrating compliance/implementation in each of the above areas, as follows:

- Program Readiness

Analysis and Control

Documentation

Training Program 
TWR-3721 Rev. 0

- Personnel Readiness

Staffing

Training

Key Personnel

- Hardware Readiness

TWRS Facilities (DSTs, 204-AR, etc.)

The assessment criteria are to identify how compliance with each of the necessary requirements is demonstrated (e.g., through a CSER, an Operating Procedure, Training Records, etc.).

Therefore, the criteria are the requirements themselves as listed in the source documents.

The assessment methodology will be primarily observation and record review and shall be documented as a map of the list of documents that demonstrate requirement compliance to the elements of readiness identified in the performance objectives. The assessment team will also perform a review of the map to ensure that the TWRS Criticality Safety Program implements the appropriate controls for each TWRS facility. 
TWR-3721 Rev. 0

This page intentionally left blank 
TWR-3721 Rev. 0

\section{APPENDIX B}

Matrix of TWRS--Specific Requirements to Implementing Documents 
TWR-3721 Rev. 0

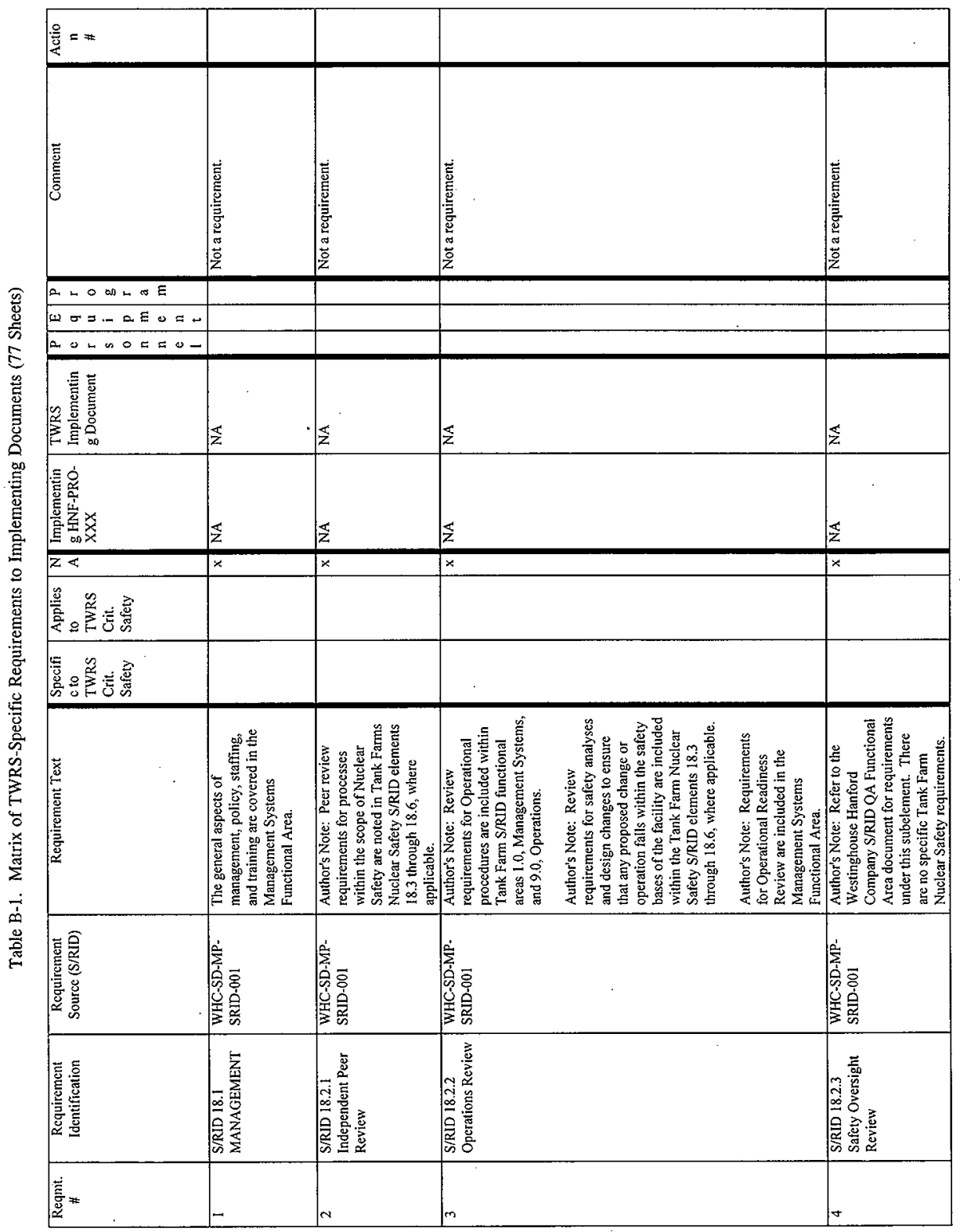

Page B-2 


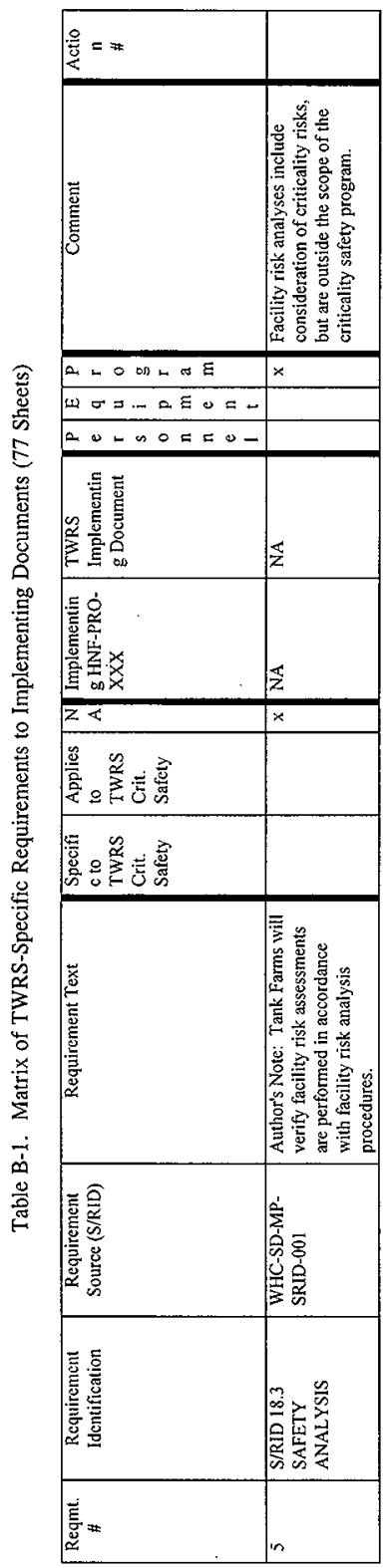

Page B-3 
$\stackrel{0}{0}=7$

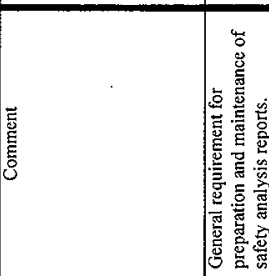

a the

H $\sigma=-2 \equiv 0=+$

EONOEAQ-

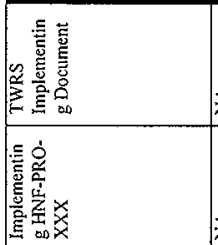

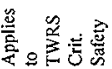

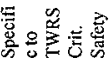

\begin{tabular}{|c|c|}
\hline 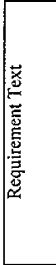 & 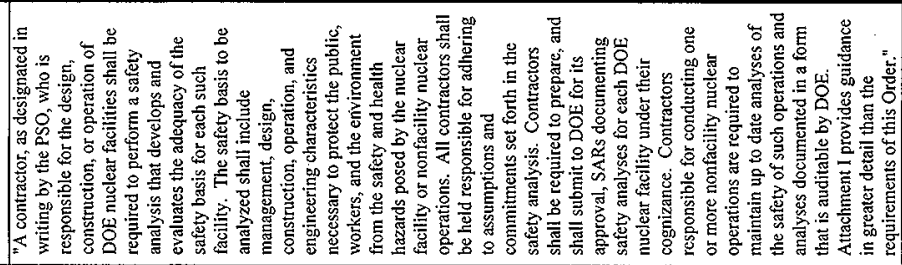 \\
\hline 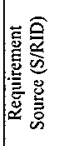 & 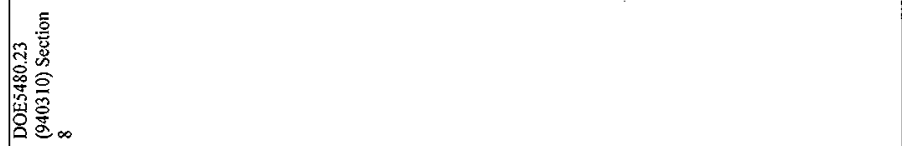 \\
\hline 童 & 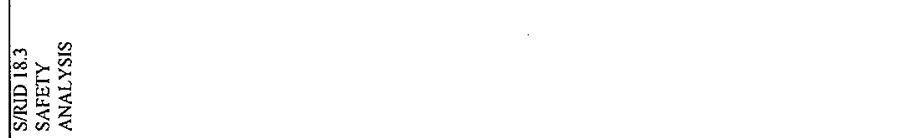 \\
\hline $\overrightarrow{\vec{g}}_{\mathscr{2}}$ & 10 \\
\hline
\end{tabular}

Page B-4 
TWR-3721 Rev. 0

옹

$\frac{8}{2}=$
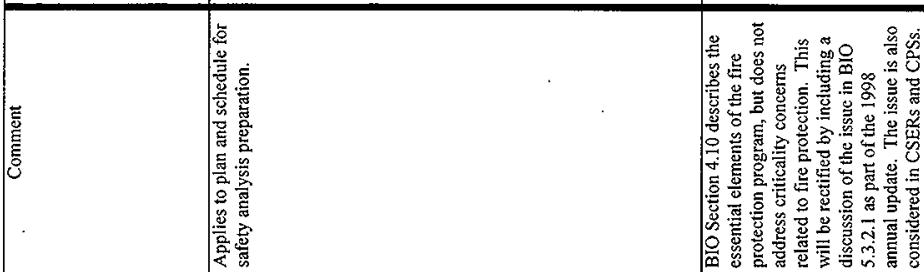

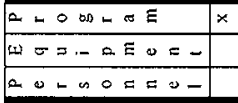

必

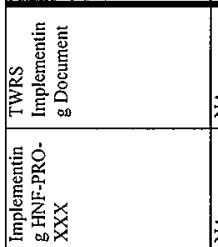

$z<$

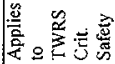

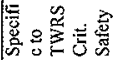

\begin{tabular}{|c|c|c|c|c|c|c|}
\hline 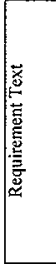 & 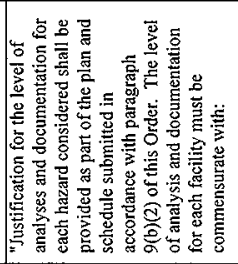 & 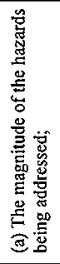 & 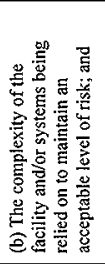 & 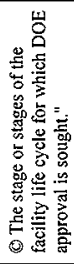 & 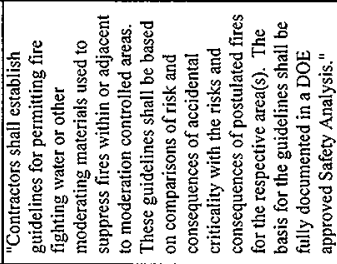 & 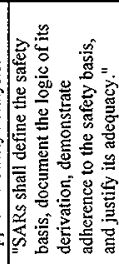 \\
\hline 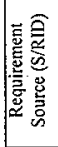 & 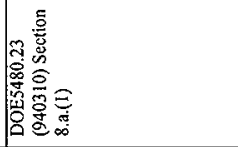 & & & & 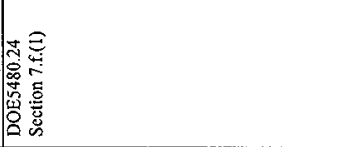 & 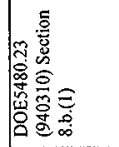 \\
\hline 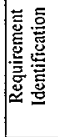 & 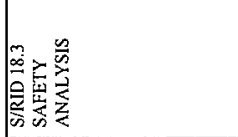 & & & & $\overbrace{\infty}^{\infty}$ & 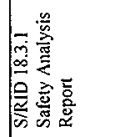 \\
\hline$\underbrace{}_{\underline{\underline{E}}}$ & or & & & & $\infty$ & 10 \\
\hline
\end{tabular}




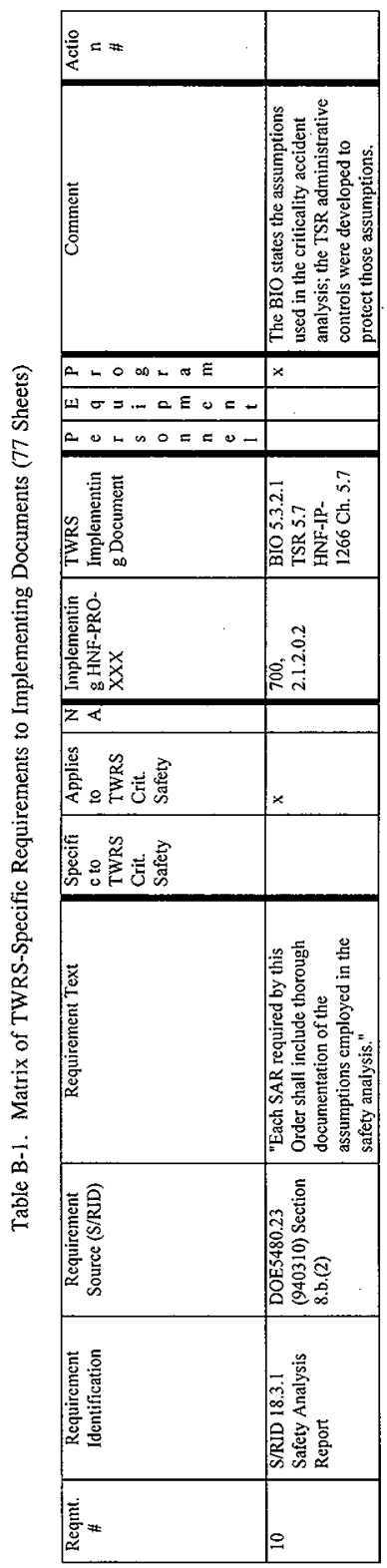

Page B-6 


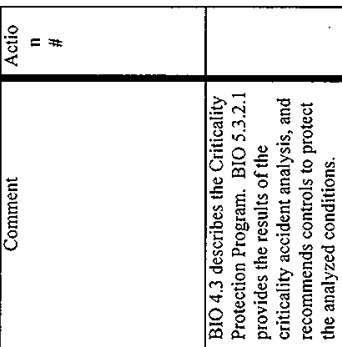

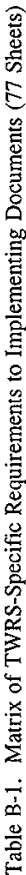

\begin{tabular}{|c|c|}
\hline$A=0$ os 2 L O $E$ & $x$ \\
\hline 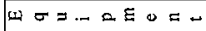 & \\
\hline A $0+\infty 0=\approx 0-$ & \\
\hline 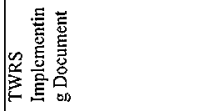 & 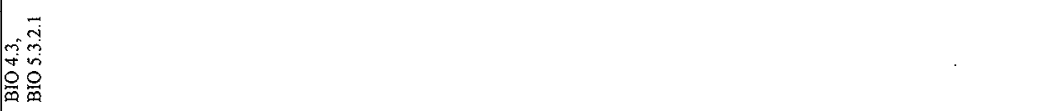 \\
\hline 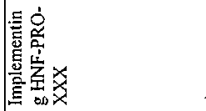 & $\frac{2}{8}$ \\
\hline$z<$ & \\
\hline 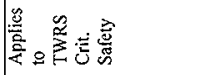 & $x$ \\
\hline 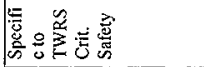 & 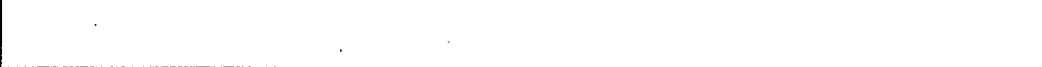 \\
\hline 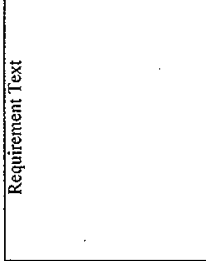 & 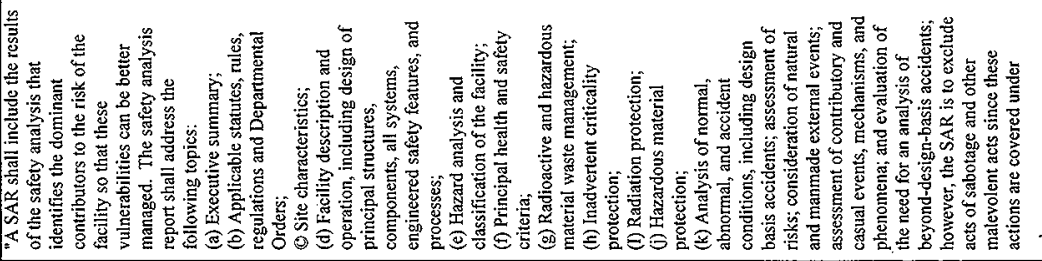 \\
\hline 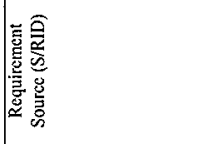 & 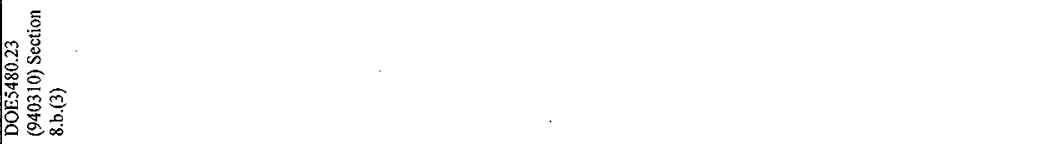 \\
\hline 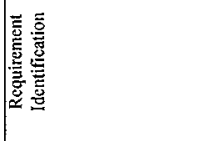 & 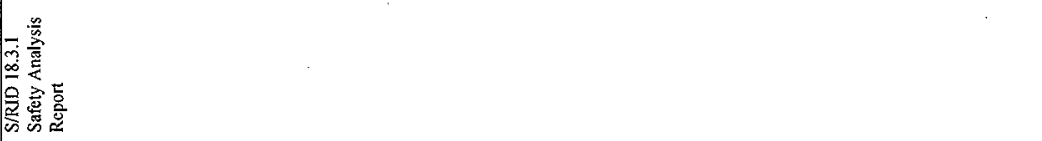 \\
\hline 豙 & $=$ \\
\hline
\end{tabular}


TWR-3721 Rev. 0

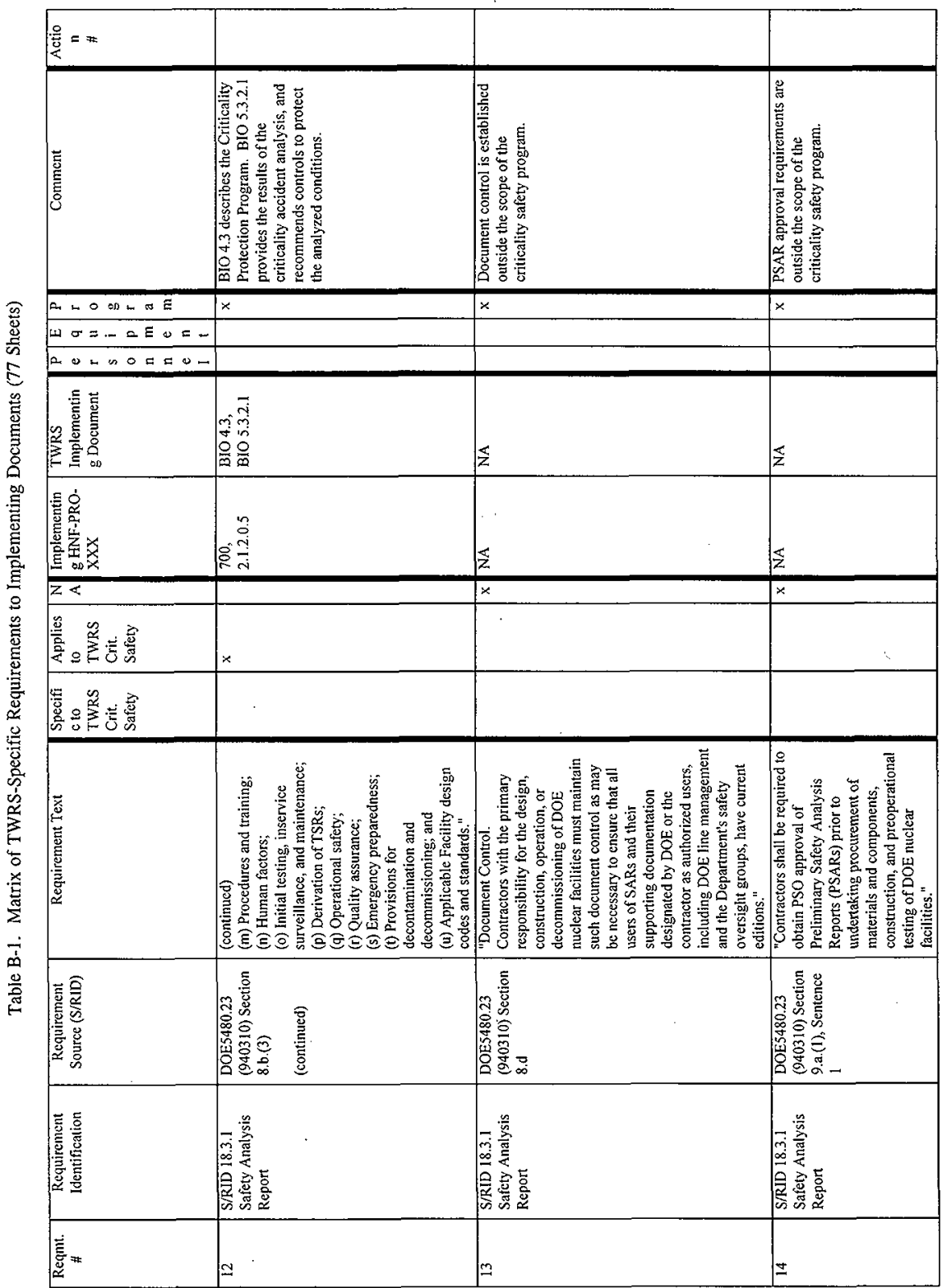


TWR-3721 Rev. 0

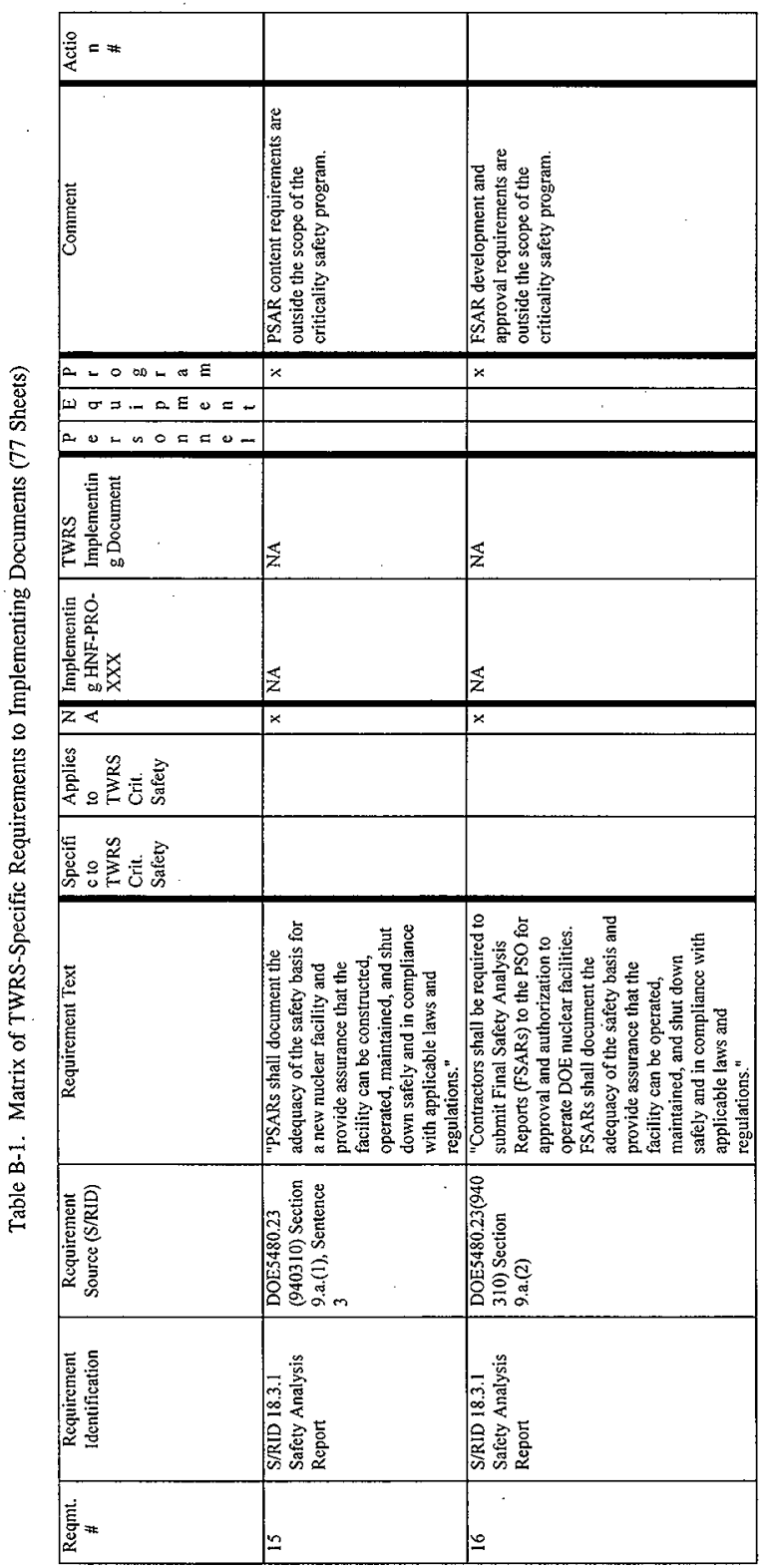

Page B-9 
递 $=$

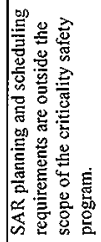

\section{$A=0$ bD $4 E$ \\ 피 $\sigma=0$ E $0=-$ \\ a. $0+$ U 0 E}

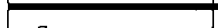

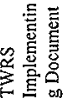

至

要

美空学

$z \varangle$

参参密总

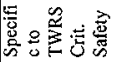

\begin{tabular}{|c|c|}
\hline 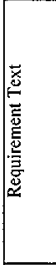 & 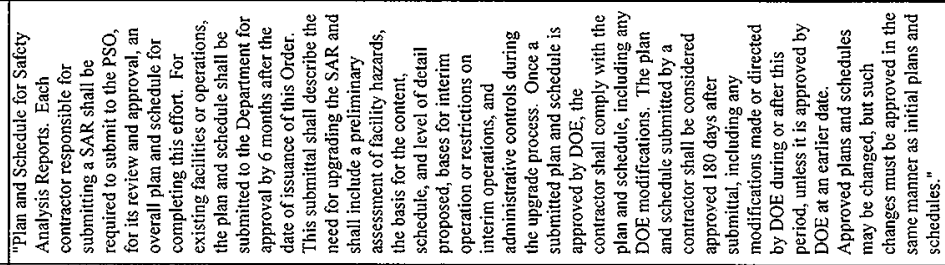 \\
\hline 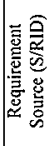 & 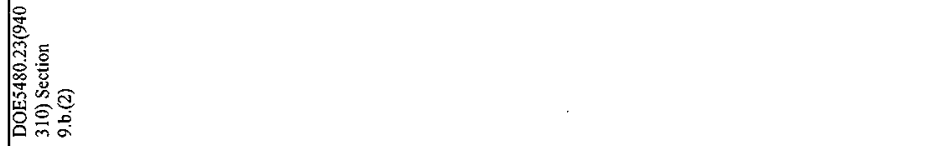 \\
\hline 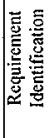 & 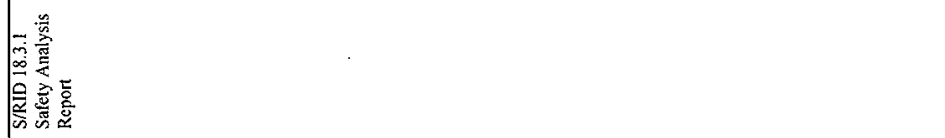 \\
\hline 节 & $1=$ \\
\hline
\end{tabular}


TWR-3721 Rev. 0

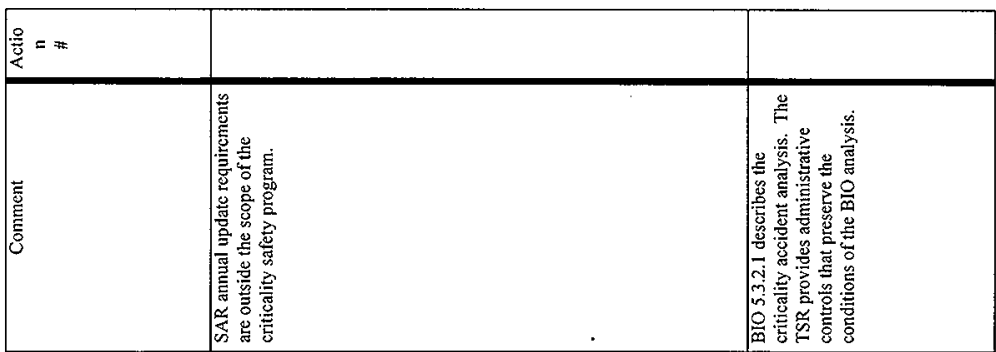

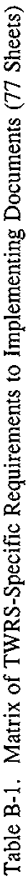

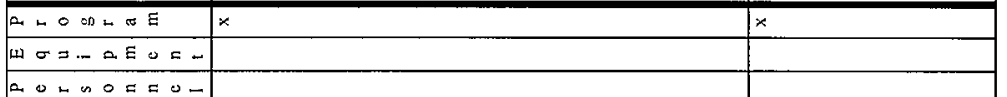

\begin{tabular}{|c|c|c|}
\hline 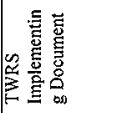 & $\frac{\pi}{z}$ & 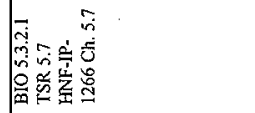 \\
\hline 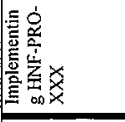 & $z$ & 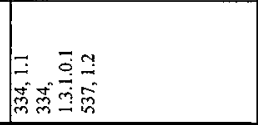 \\
\hline$z<$ & $x$ & \\
\hline 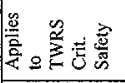 & & \\
\hline 言事离 & & $\times$ \\
\hline 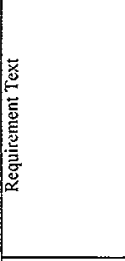 & 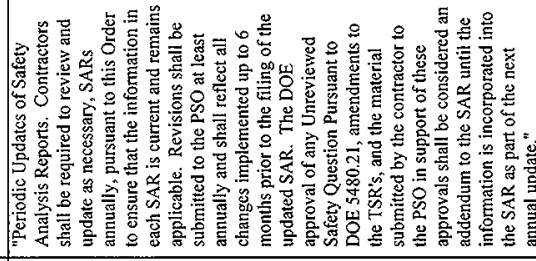 & 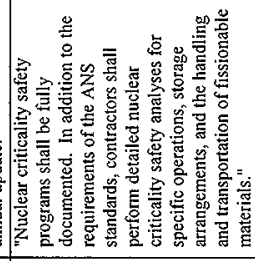 \\
\hline 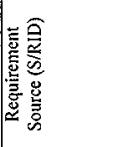 & 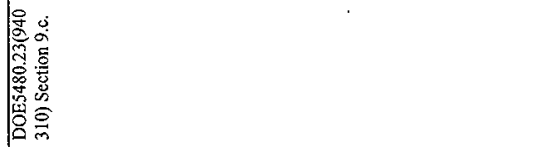 & 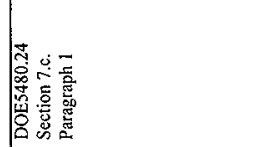 \\
\hline 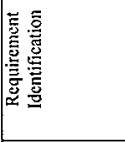 & 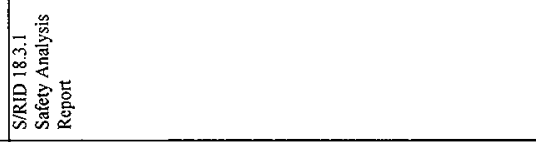 & 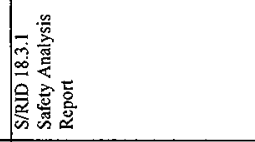 \\
\hline 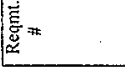 & $\stackrel{\infty}{\infty}$ & 9 \\
\hline
\end{tabular}




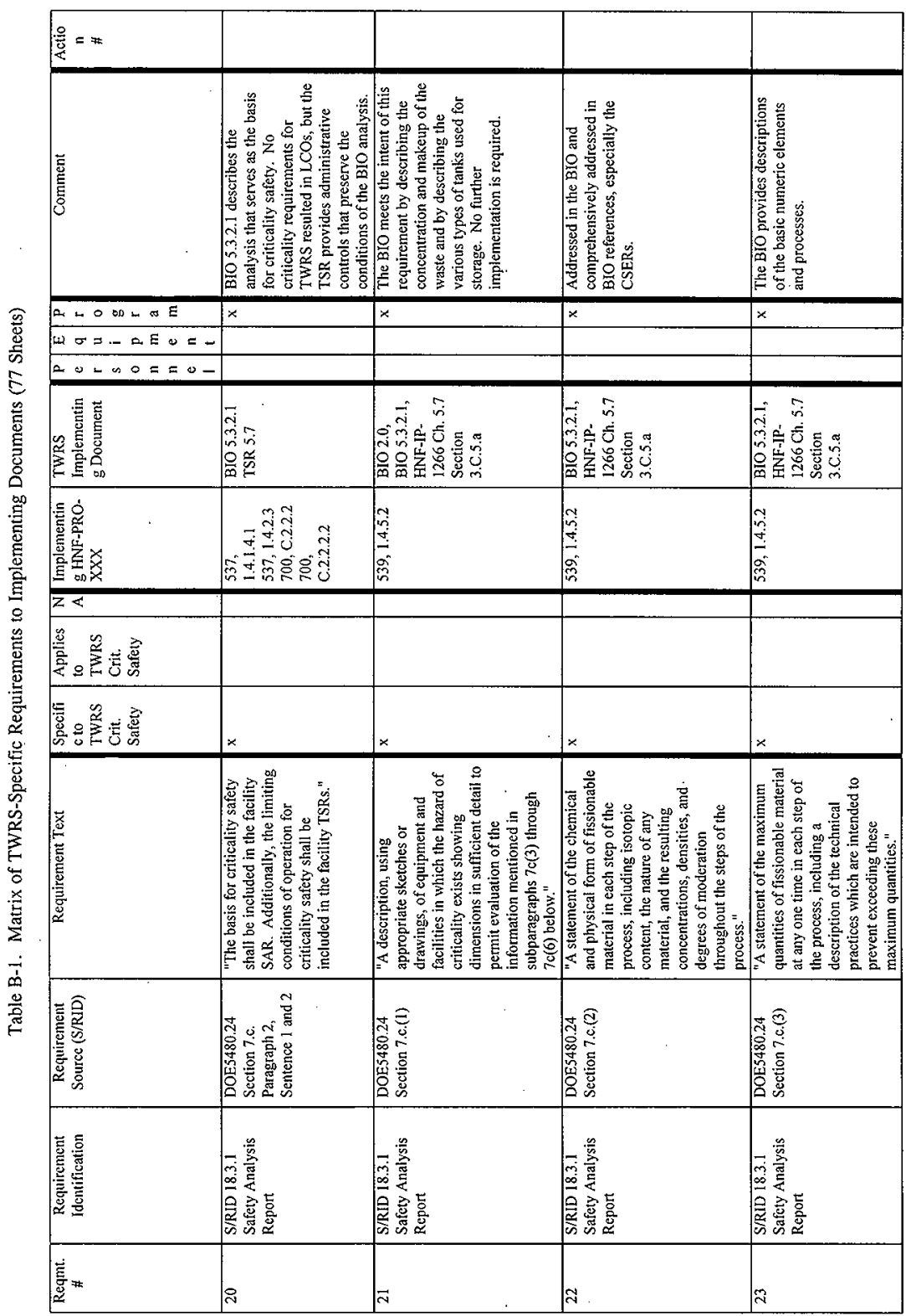


TWR-3721 Rev. 0

\begin{tabular}{|c|c|c|c|}
\hline$\left\{\begin{array}{l}0 \\
0\end{array}\right.$ & & & \\
\hline 信 & 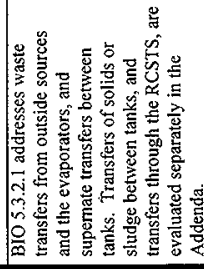 & 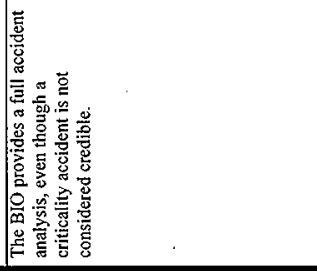 & 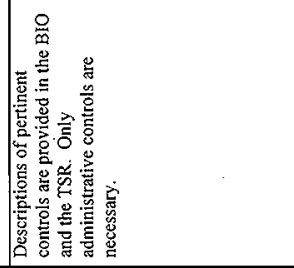 \\
\hline OLOO & $x$ & $x$. & 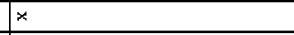 \\
\hline$\Phi \sigma \exists \ldots a \equiv 0=-$ & & & \\
\hline $0 \cup \operatorname{nos} 0=00-$ & & & \\
\hline 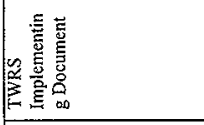 & 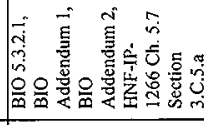 & 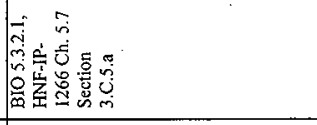 & 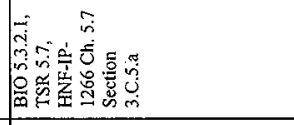 \\
\hline 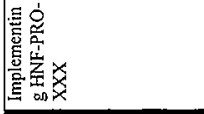 & 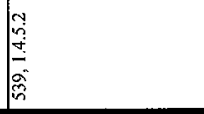 & 哭 & 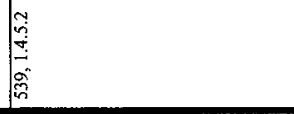 \\
\hline$z<$ & & & \\
\hline 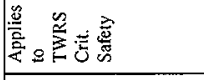 & & & \\
\hline 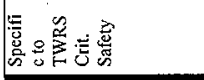 & $x$ & $x$ & $x$ \\
\hline 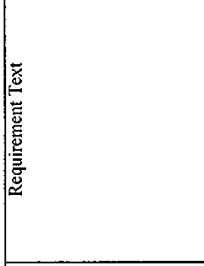 & 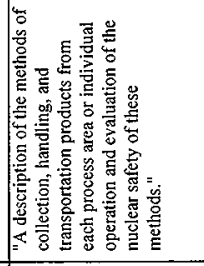 & 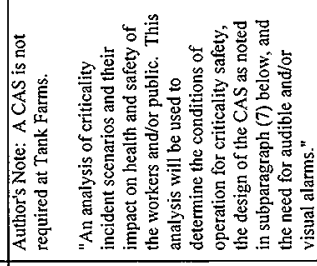 & 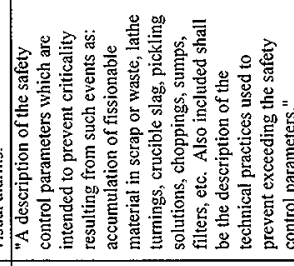 \\
\hline 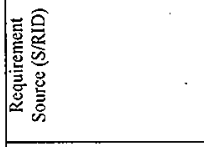 & 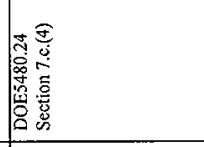 & 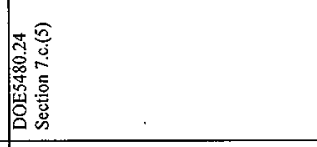 & 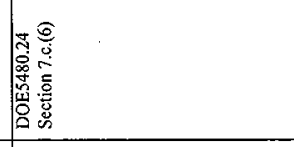 \\
\hline 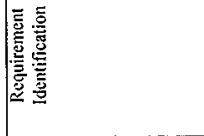 & 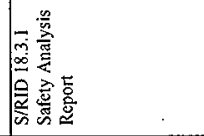 & 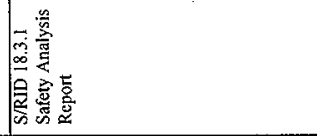 & 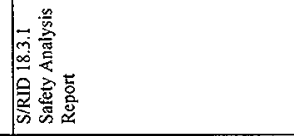 \\
\hline 言 & $\pi$ & $\approx$ & 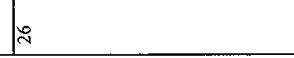 \\
\hline
\end{tabular}




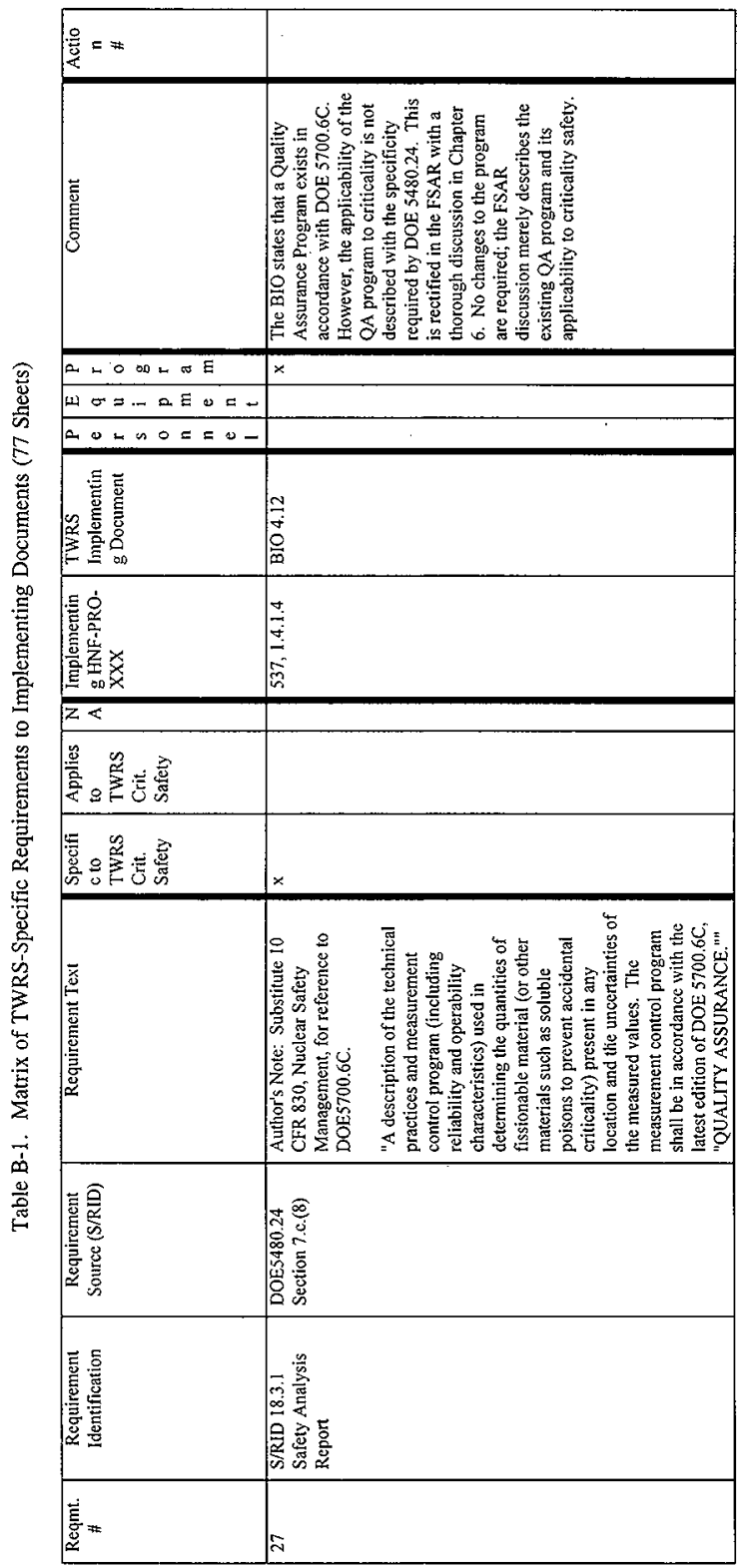

Page B-14 
TWR-3721 Rev. 0

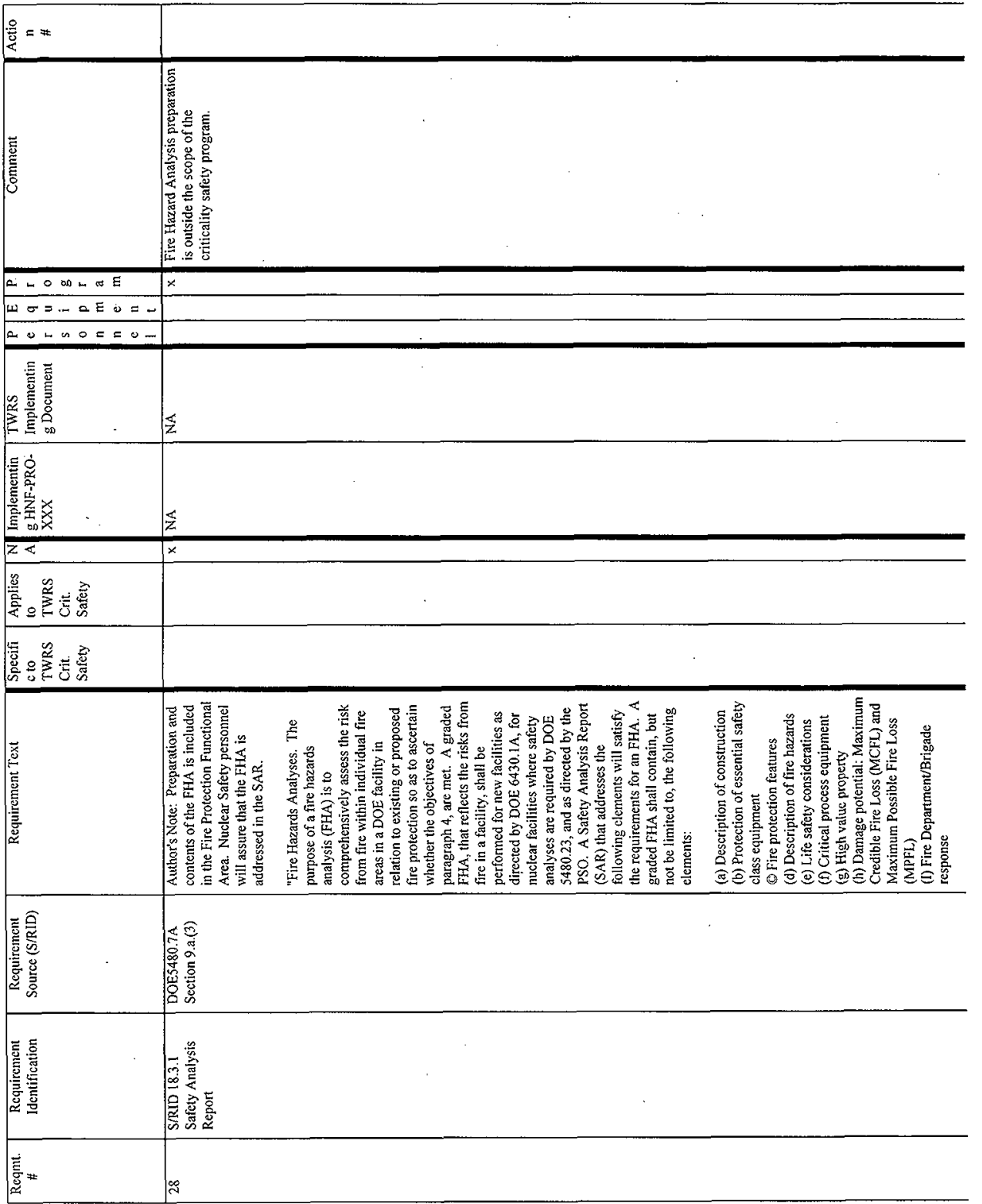

Page B-15 
TWR-3721 Rev. 0

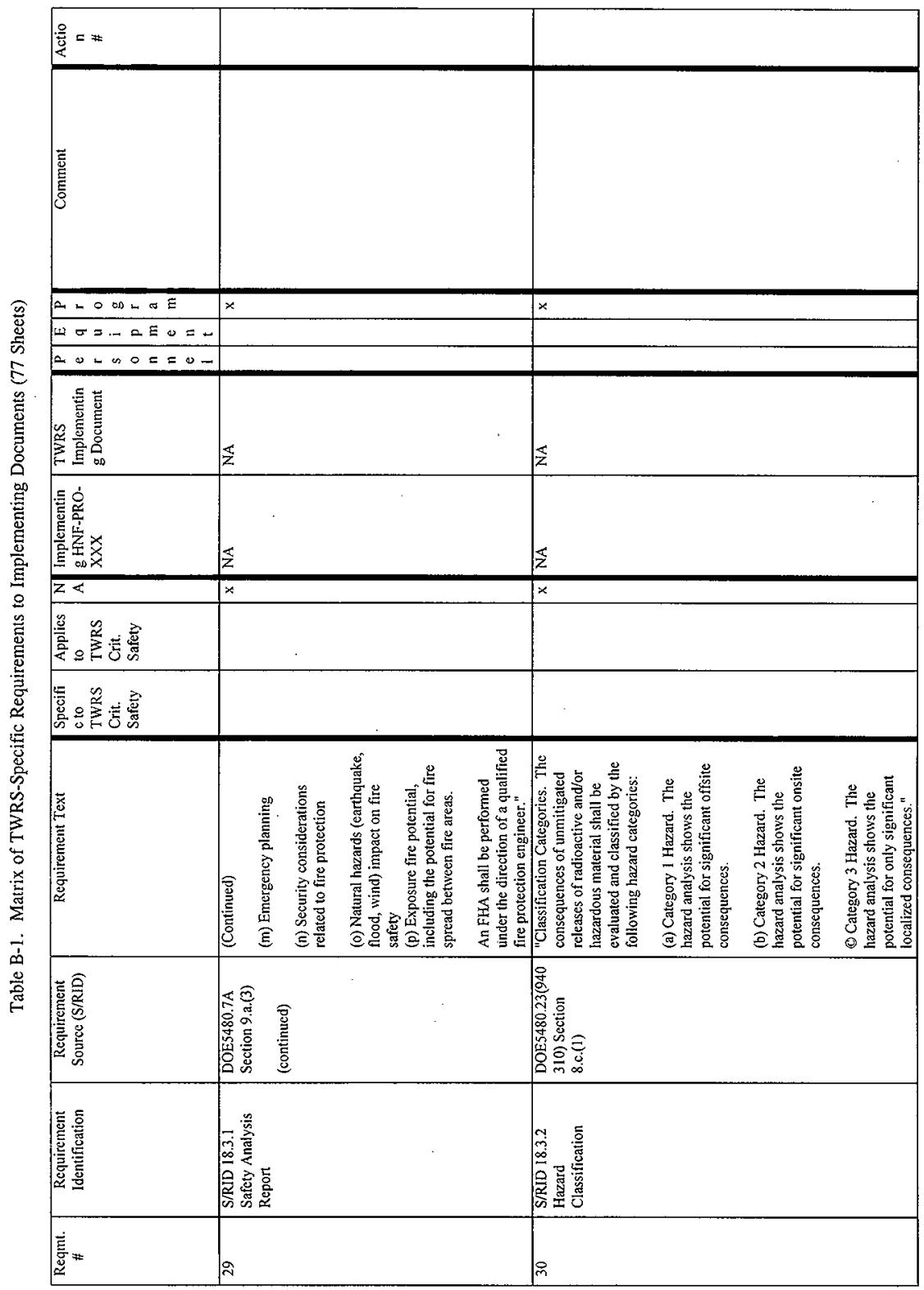

Page B-16 
TWR-3721 Rev. 0

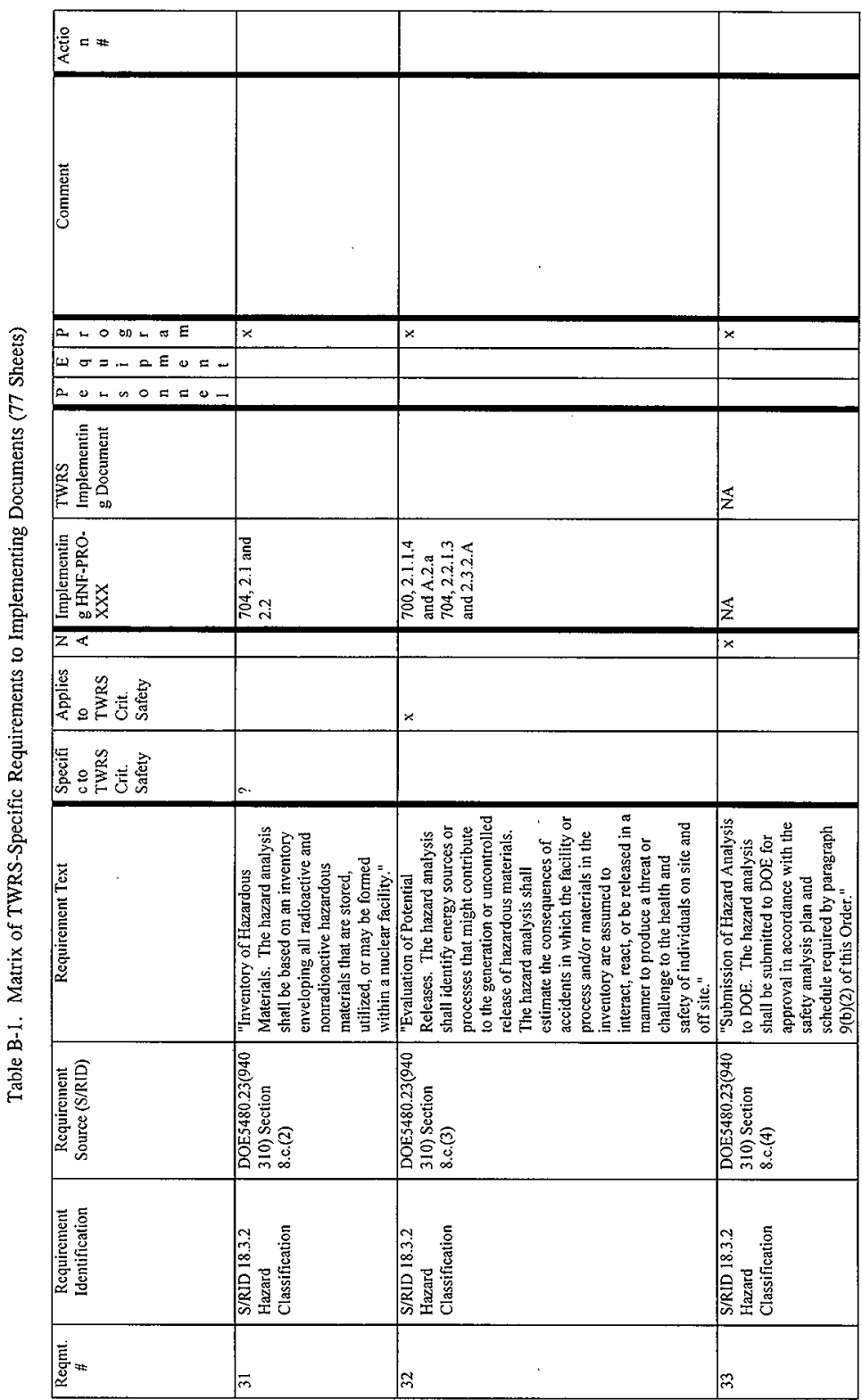




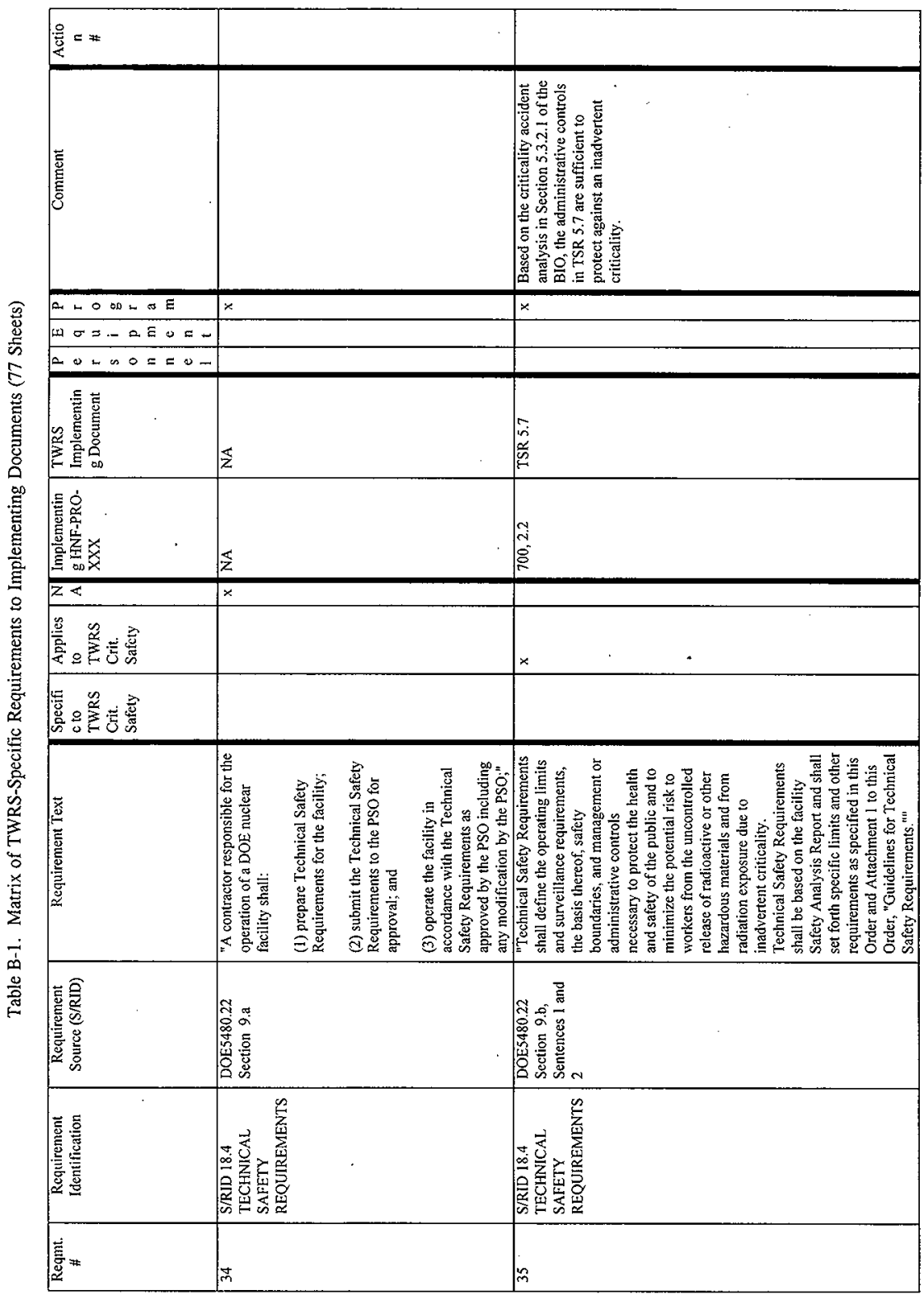




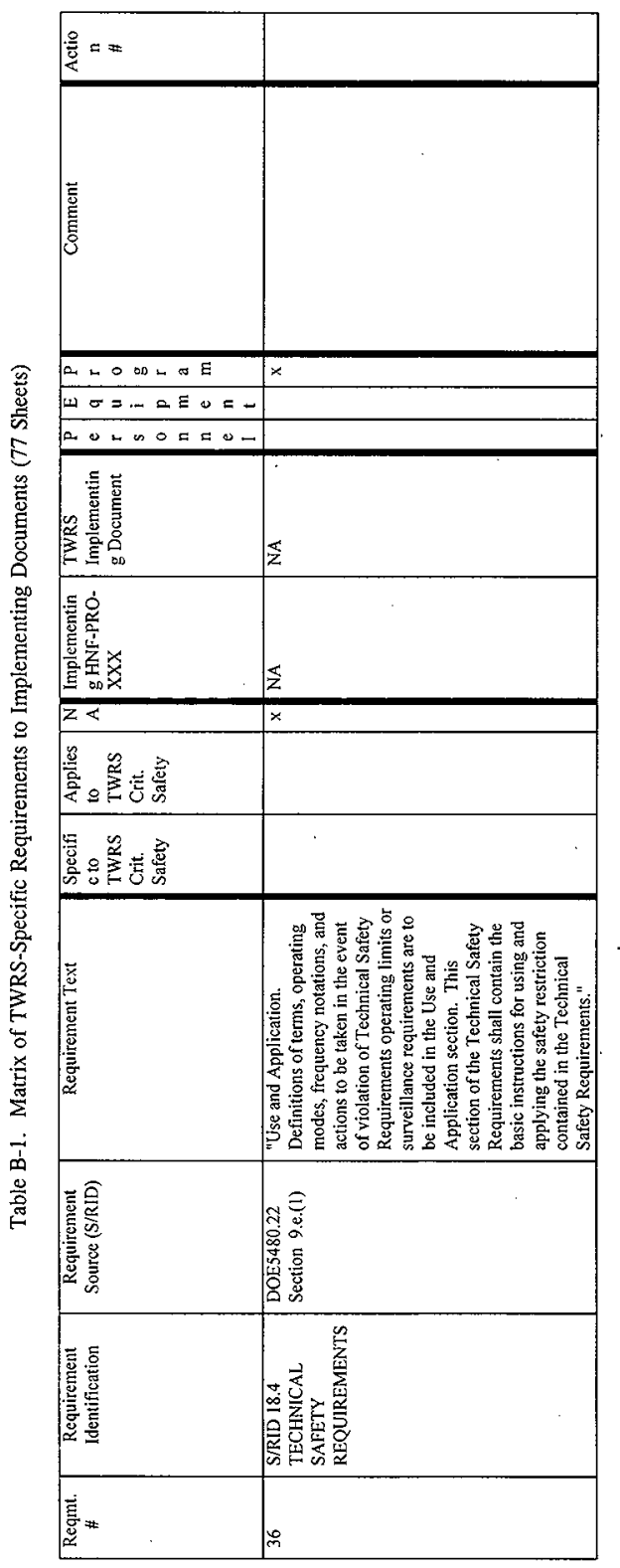

Page B-19 


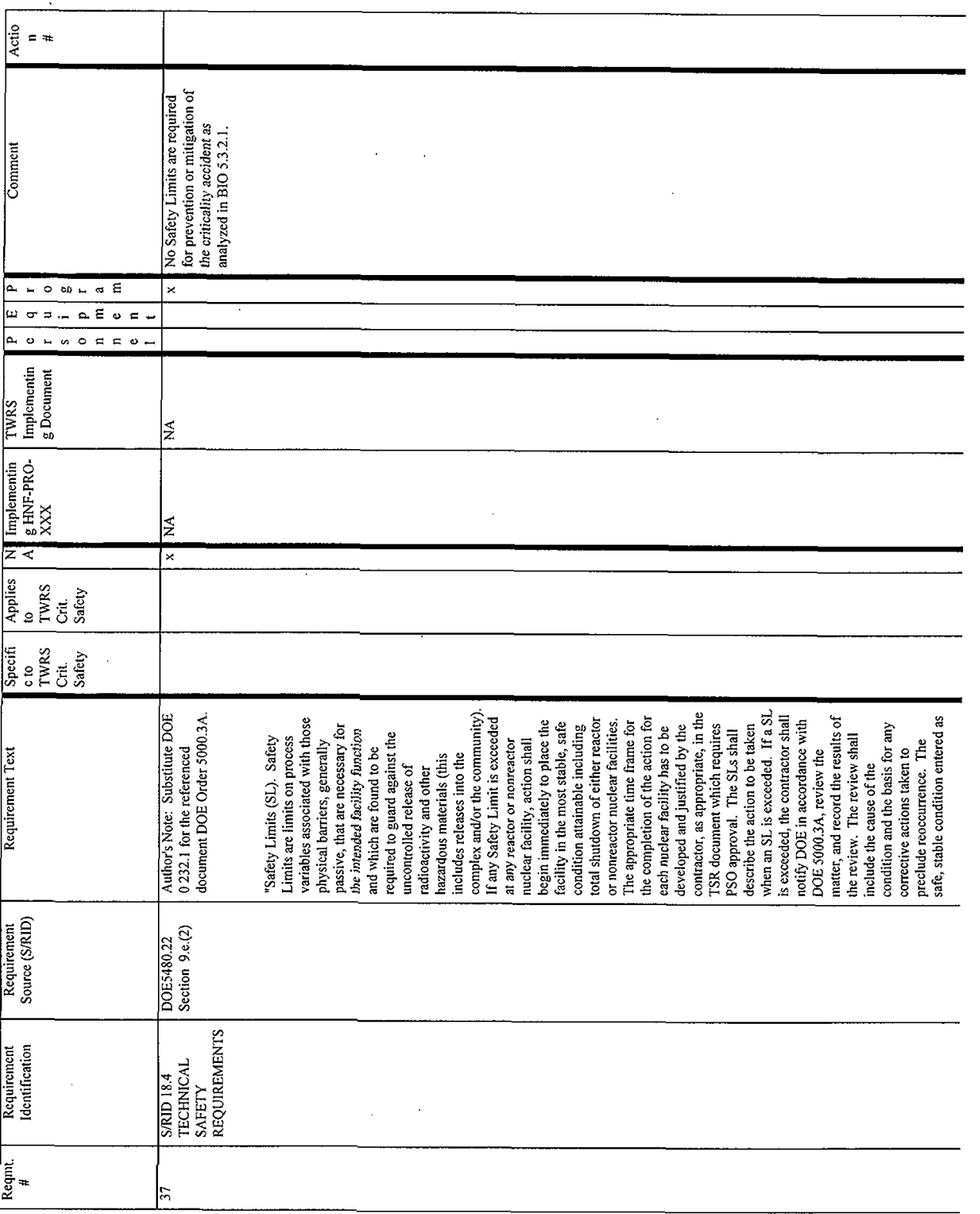




\begin{tabular}{|c|c|}
\hline$\frac{0}{0}=7$ & \\
\hline 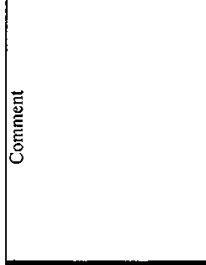 & 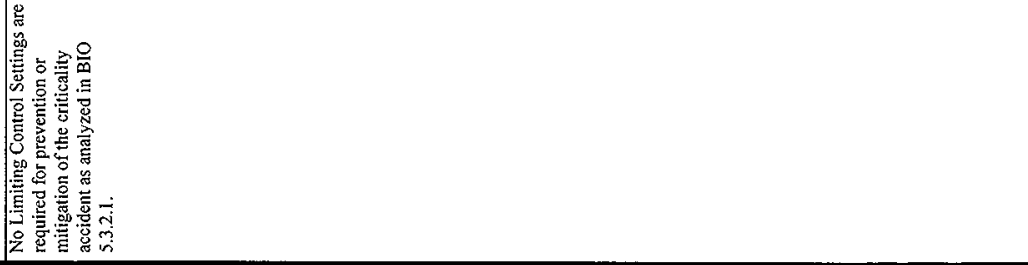 \\
\hline$A \leftarrow 0=0 \leftarrow \approx E$ & $x$ \\
\hline$\omega=\ldots \equiv 0=$ & $x$ \\
\hline $00200=0=-$ & \\
\hline 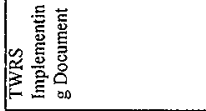 & 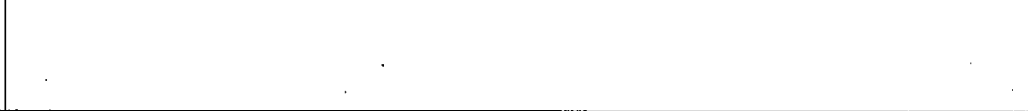 \\
\hline 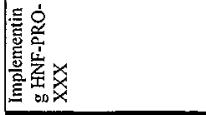 & 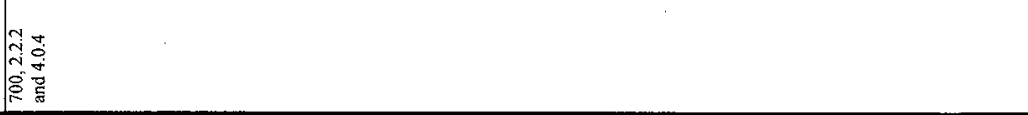 \\
\hline$z<$ & . \\
\hline 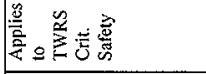 & . \\
\hline 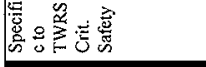 & \\
\hline 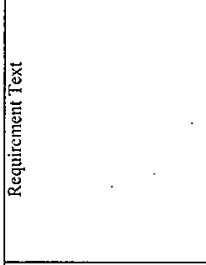 & 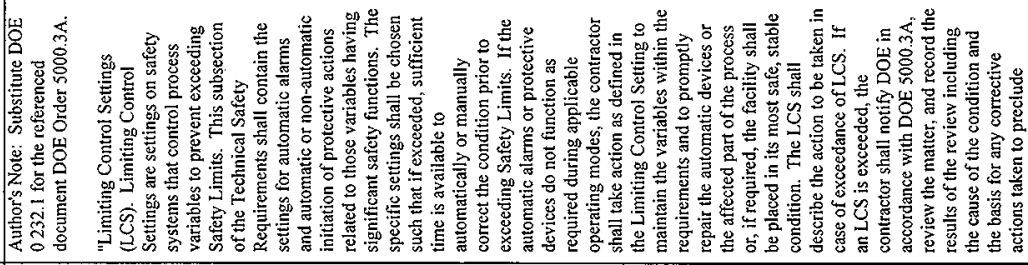 \\
\hline 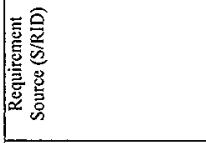 & 弪 \\
\hline 总总 & | \\
\hline 䓂 $=$ & $\infty$ \\
\hline
\end{tabular}




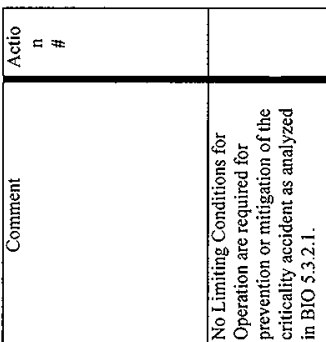

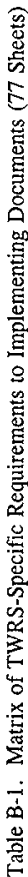

\begin{tabular}{|c|c|}
\hline \multirow{2}{*}{\multicolumn{2}{|c|}{ wa $=0$}} \\
\hline & $x$ \\
\hline
\end{tabular}

$A 0-\infty 0=E 0-$

\begin{tabular}{|c|c|}
\hline & 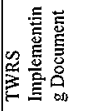 \\
\hline & 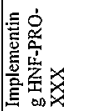 \\
\hline
\end{tabular}

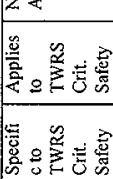

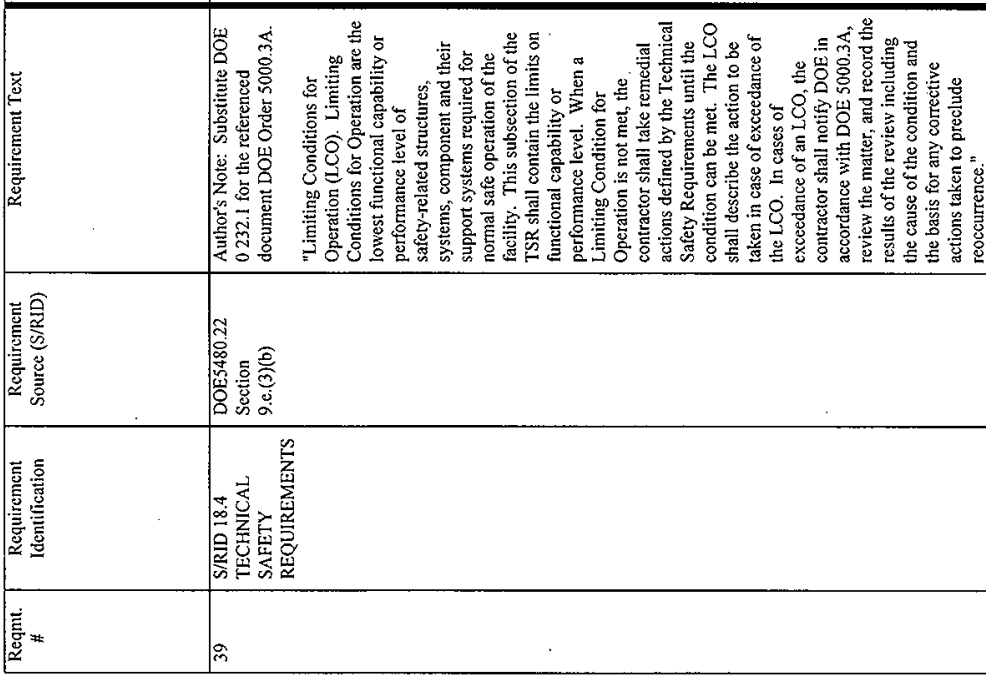




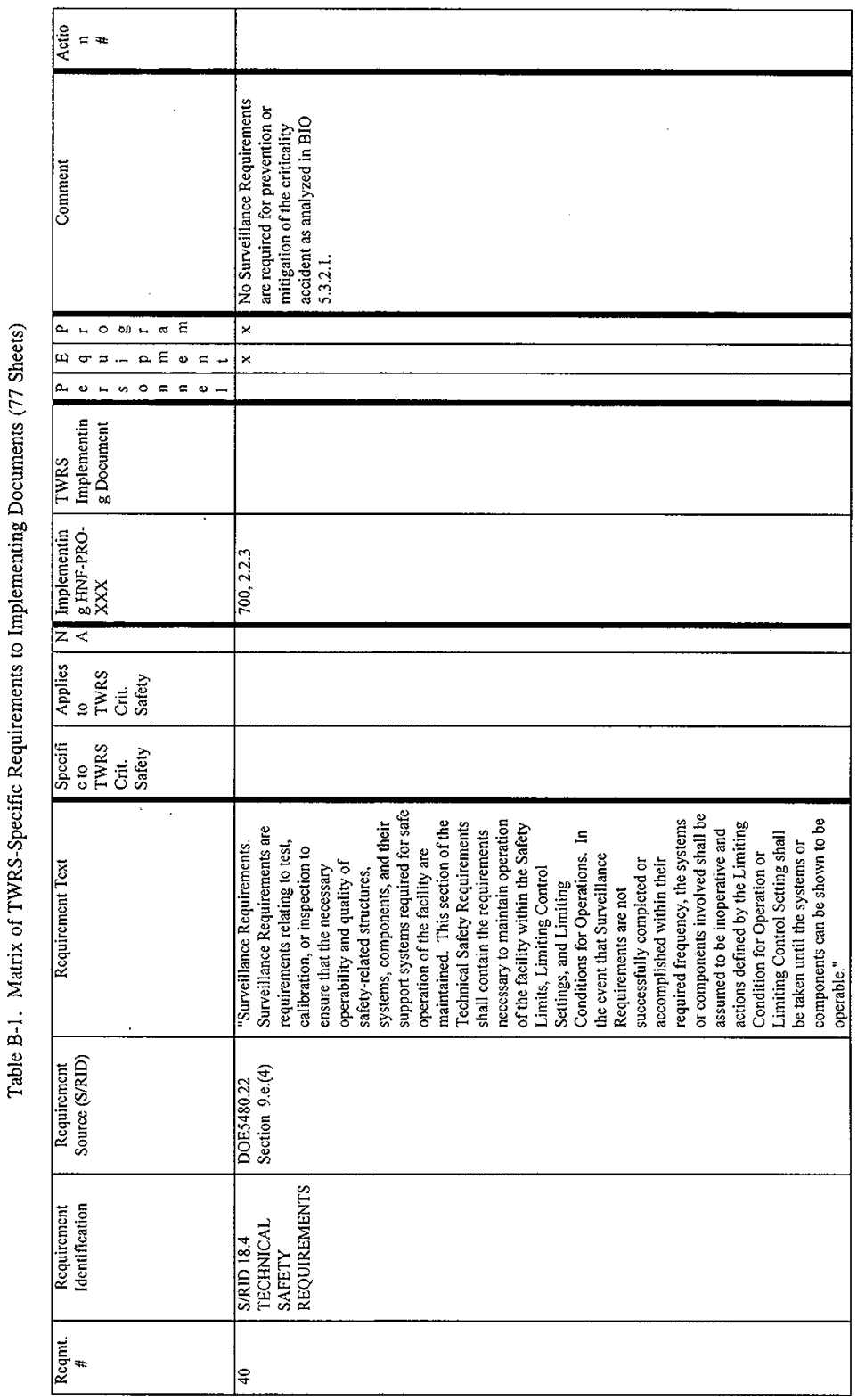

Page B-23 


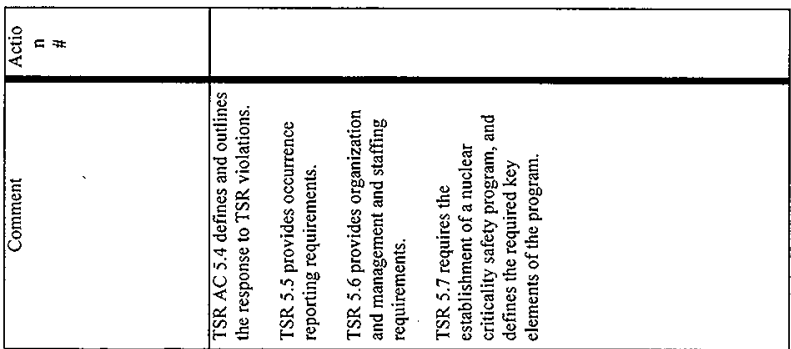

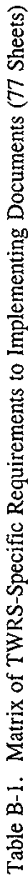

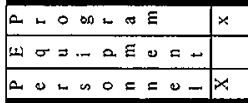

\begin{tabular}{|c|c|}
\hline 焉苛 & 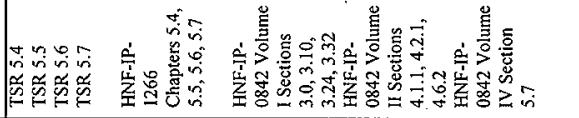 \\
\hline 焉旁 & 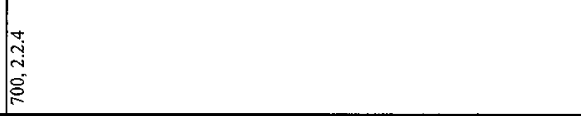 \\
\hline \multicolumn{2}{|l|}{$z<$} \\
\hline 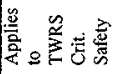 & $x$ \\
\hline \multicolumn{2}{|l|}{ 焉总总总 } \\
\hline 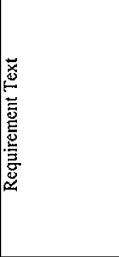 & 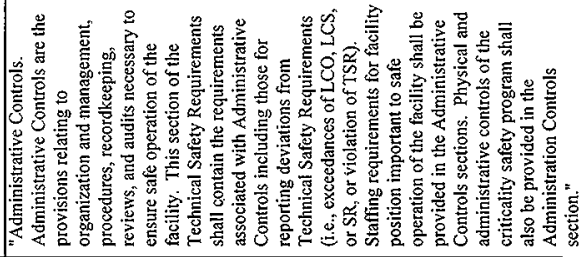 \\
\hline 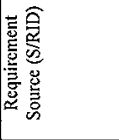 & 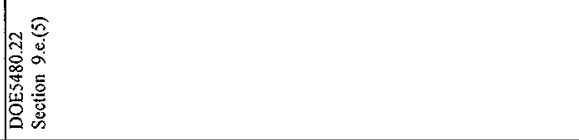 \\
\hline 竞 & | \\
\hline$\dot{\vec{g}}_{\tilde{g}}$ & $\nabla$ \\
\hline
\end{tabular}


TWR-3721 Rev. 0

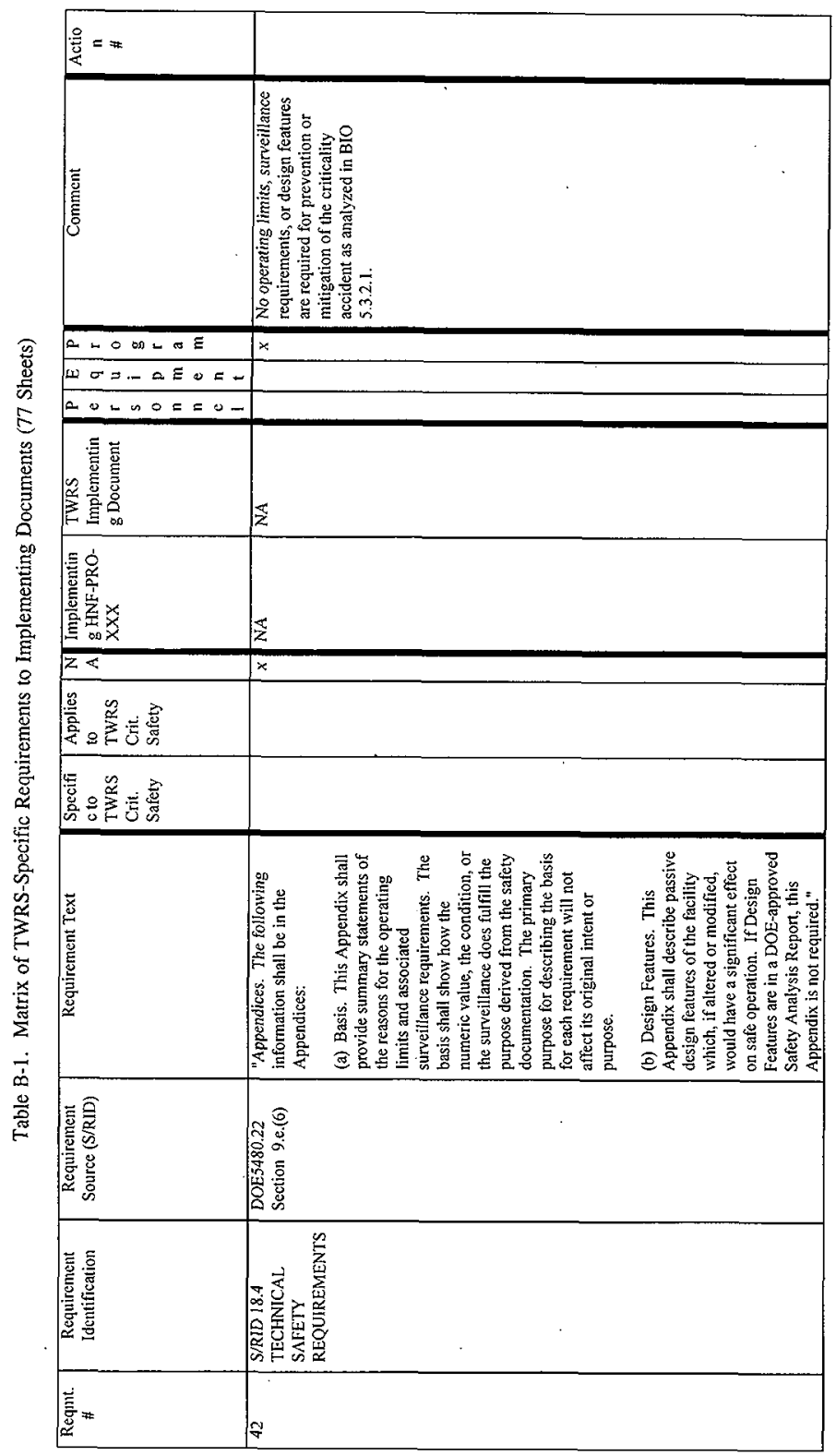

Page B-25 


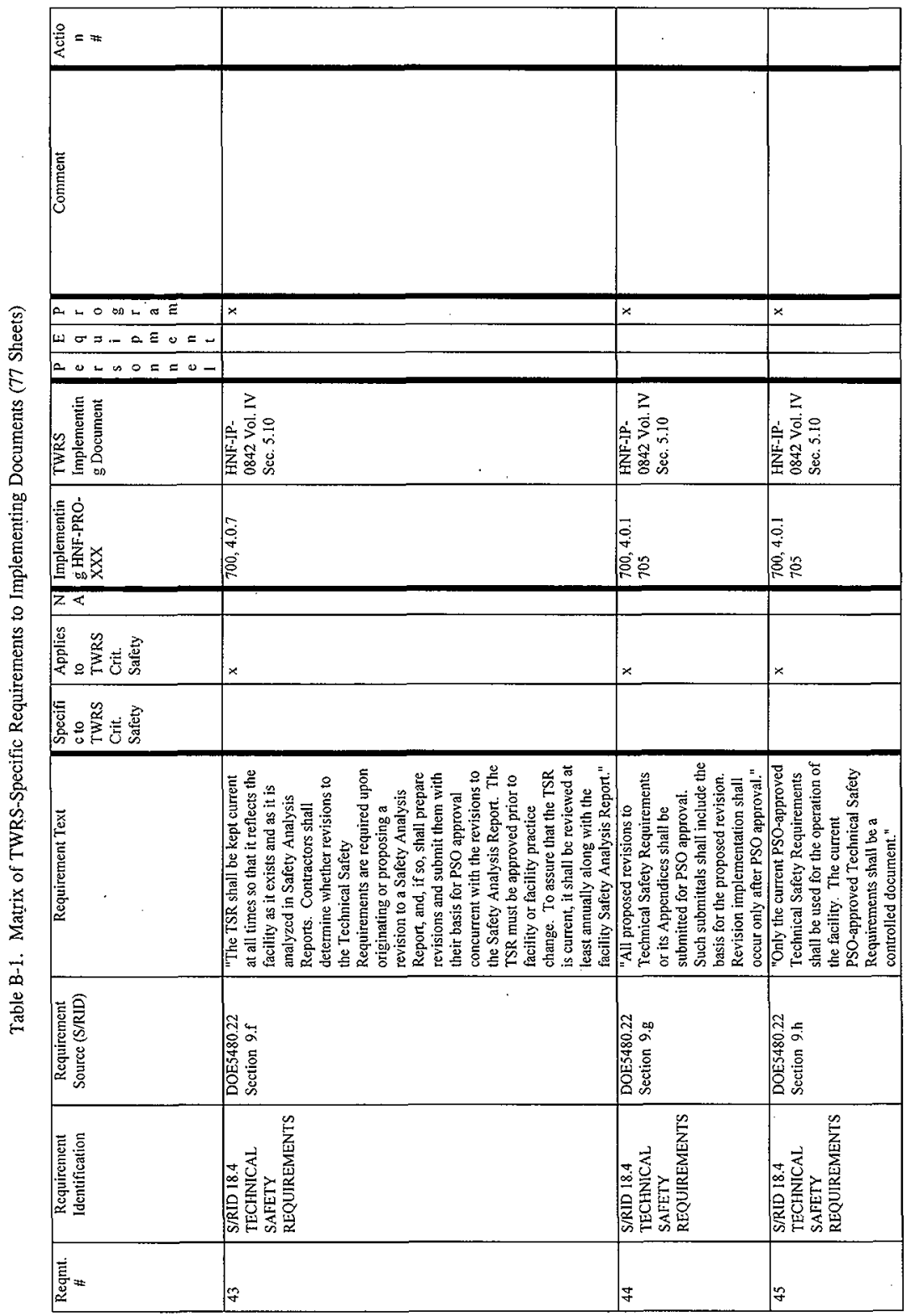

Page B-26 
TWR-3721 Rev. 0

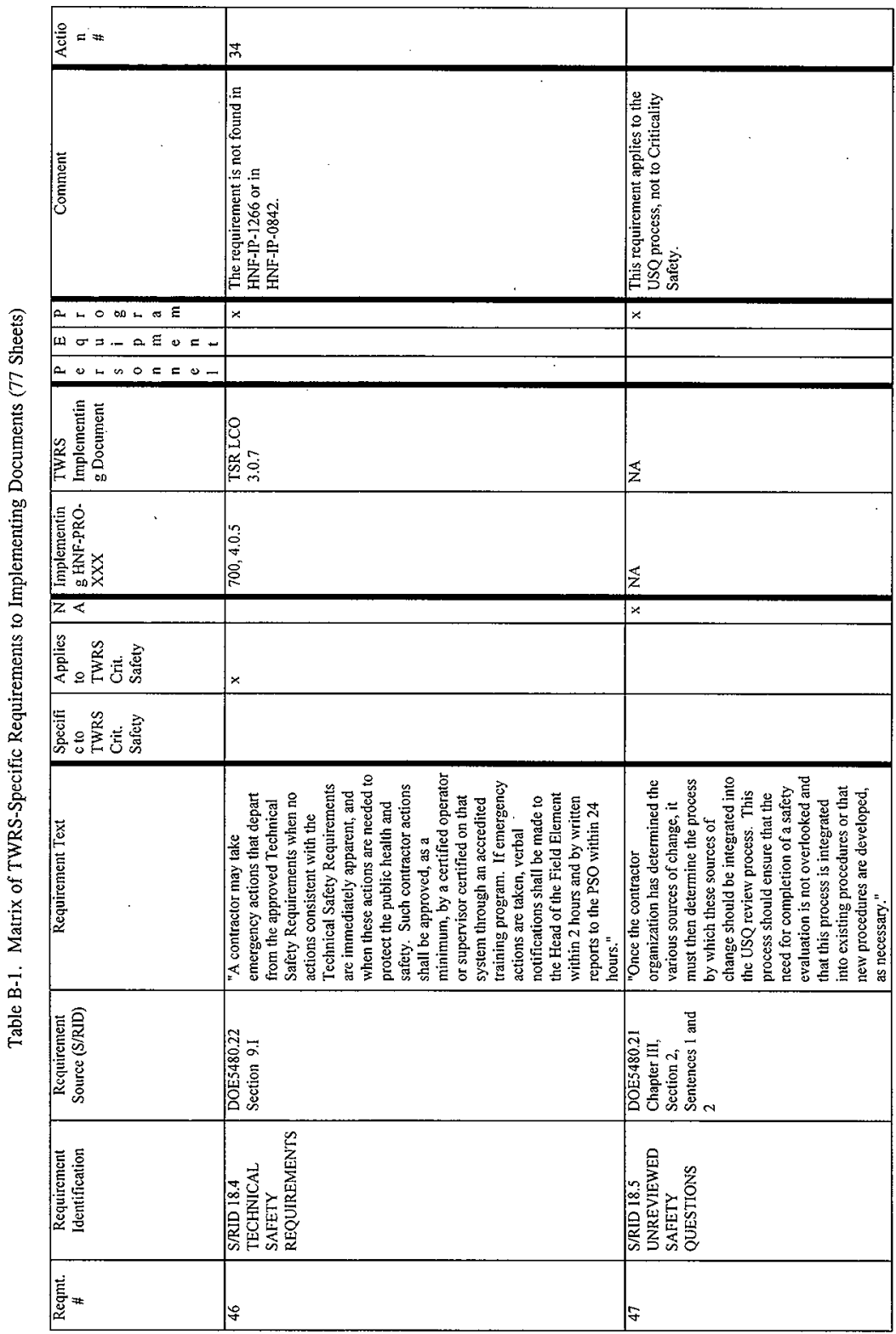

Page B-27 
TWR-3721 Rev. 0

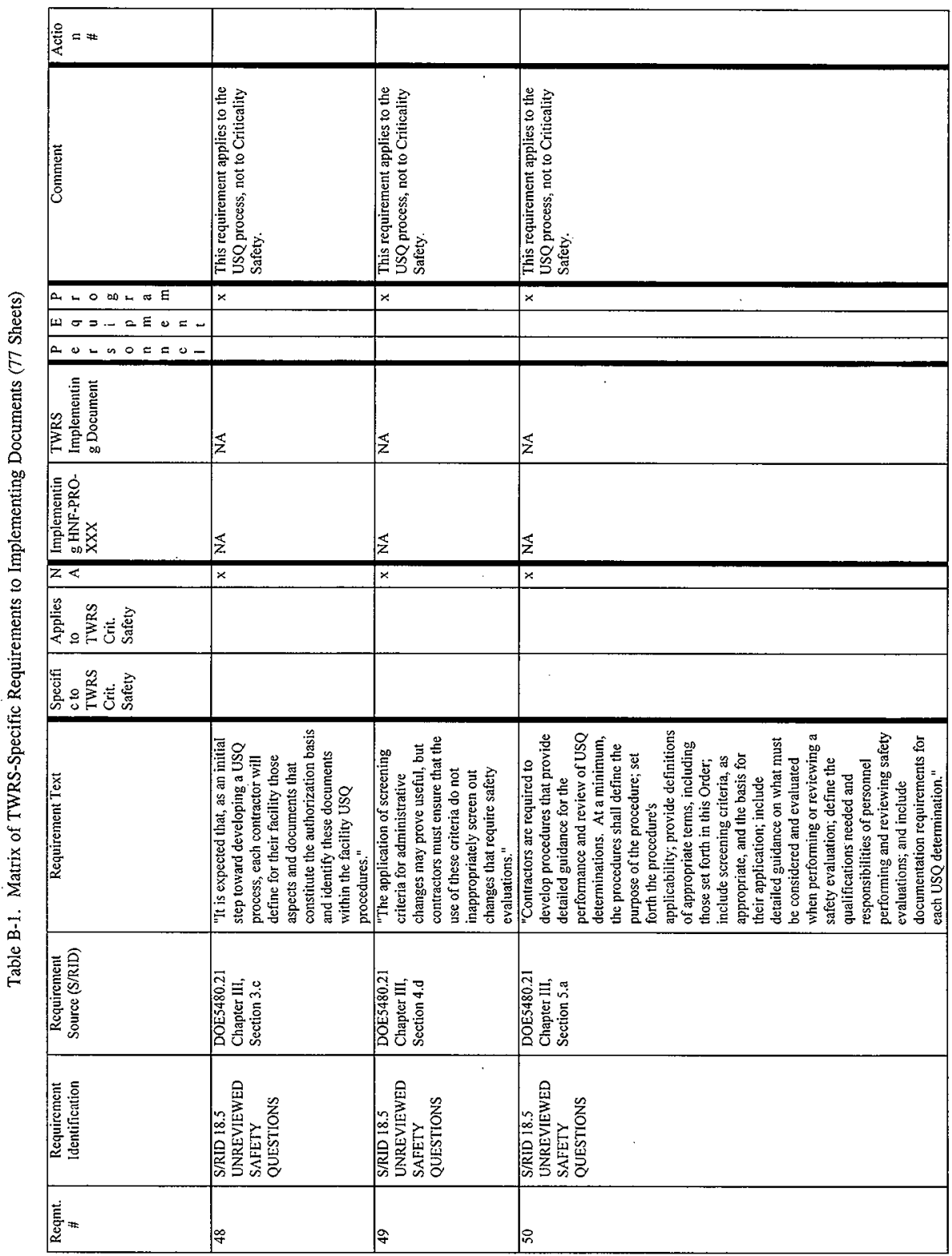

Page B-28 


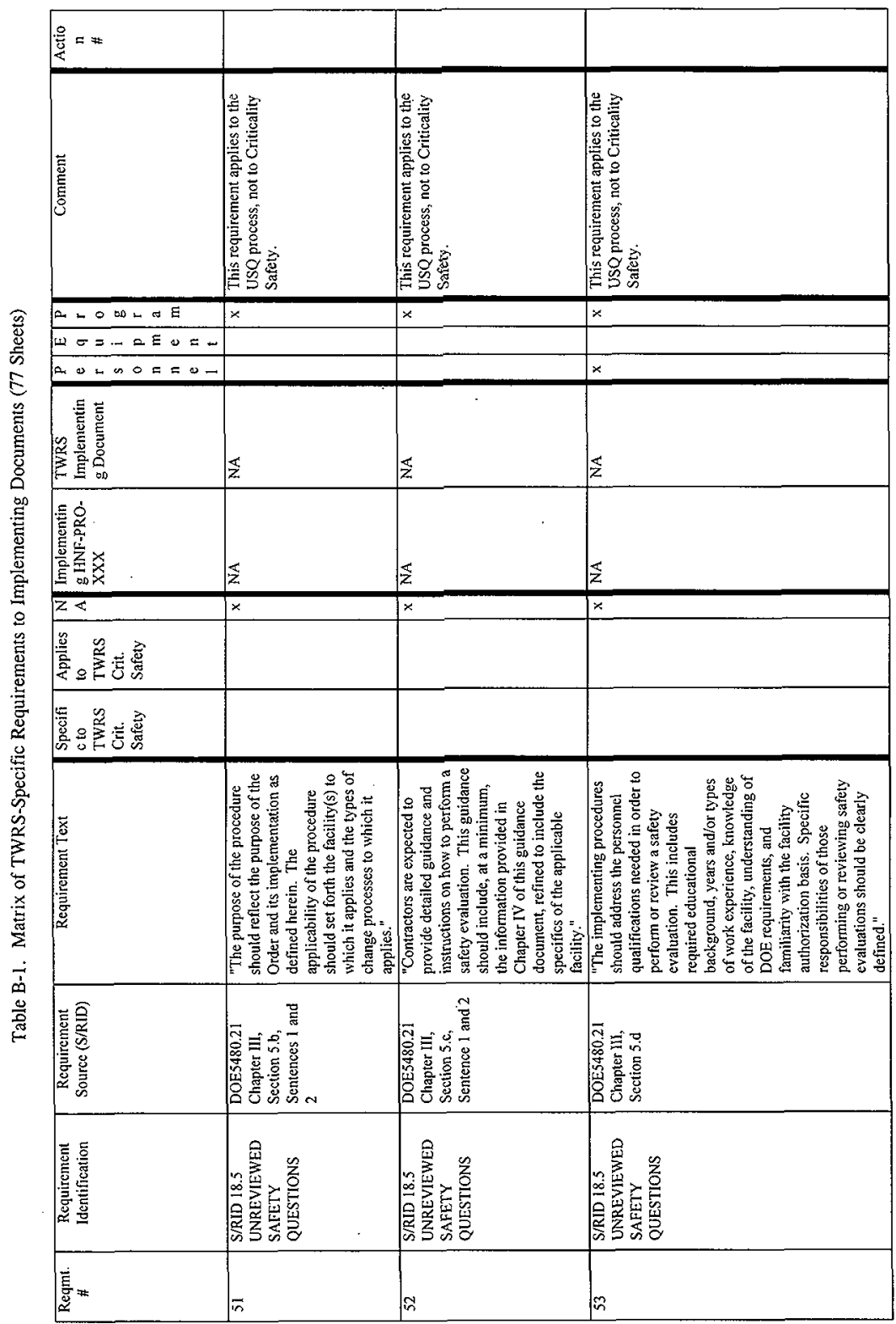

Page B-29 
TWR-3721 Rev. 0

\begin{tabular}{|c|c|c|c|}
\hline$\frac{\circ}{3}=7$ & & & \\
\hline 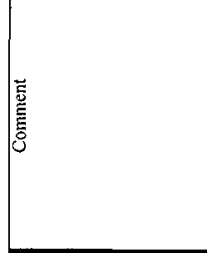 & 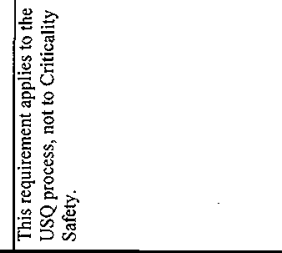 & 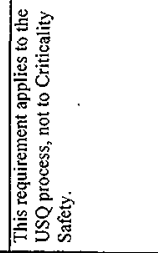 & 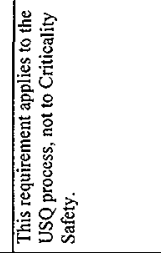 \\
\hline a $-000 \mathrm{~m} \pi \mathrm{E}$ & $x$ & & $x$ \\
\hline$\omega \sigma=\ldots 2 E 0=-$ & & & \\
\hline $00+\infty 0=20$ & & $x$ & \\
\hline 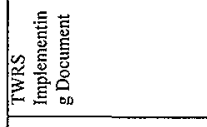 & 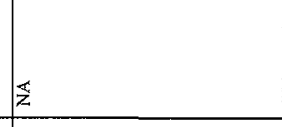 & $\Sigma$ & 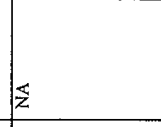 \\
\hline 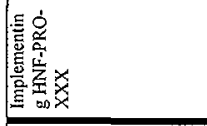 & $\frac{\pi}{z}$ & $\frac{\pi}{z}$ & $\$$ \\
\hline$z<$ & $x$ & $x$ & $x$ \\
\hline 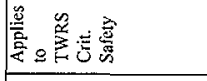 & & & \\
\hline 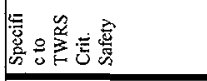 & & & \\
\hline 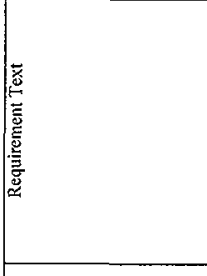 & 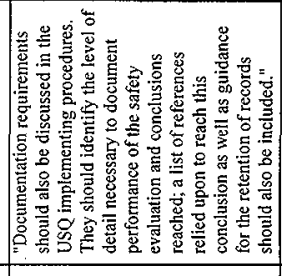 & 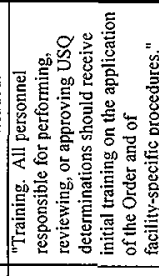 & 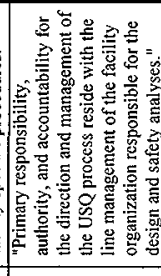 \\
\hline 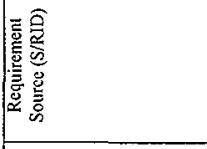 & 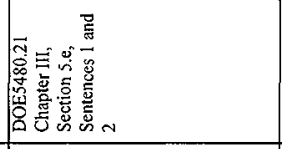 & 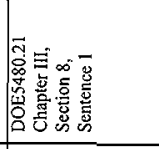 & 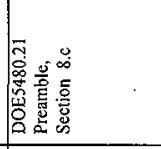 \\
\hline 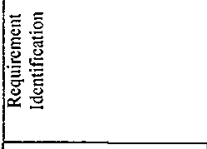 & 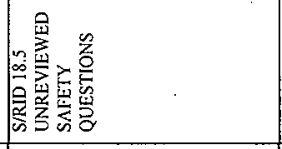 & 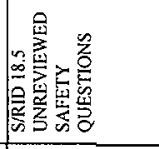 & 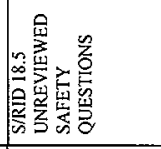 \\
\hline 掊 & In & $\approx$ & $\therefore$ \\
\hline
\end{tabular}

Page B-30 
TWR-3721 Rev. 0

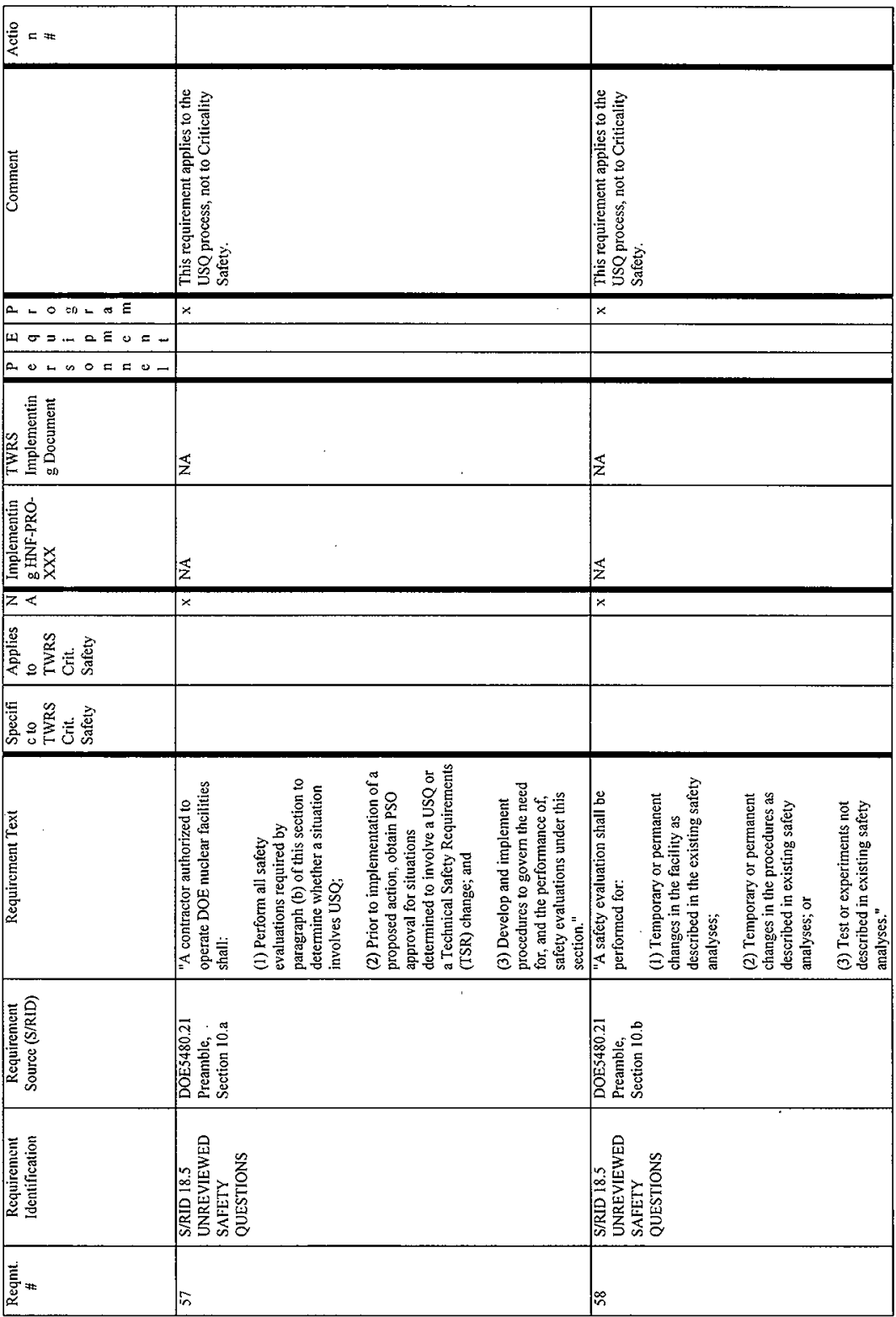

Page B-31 


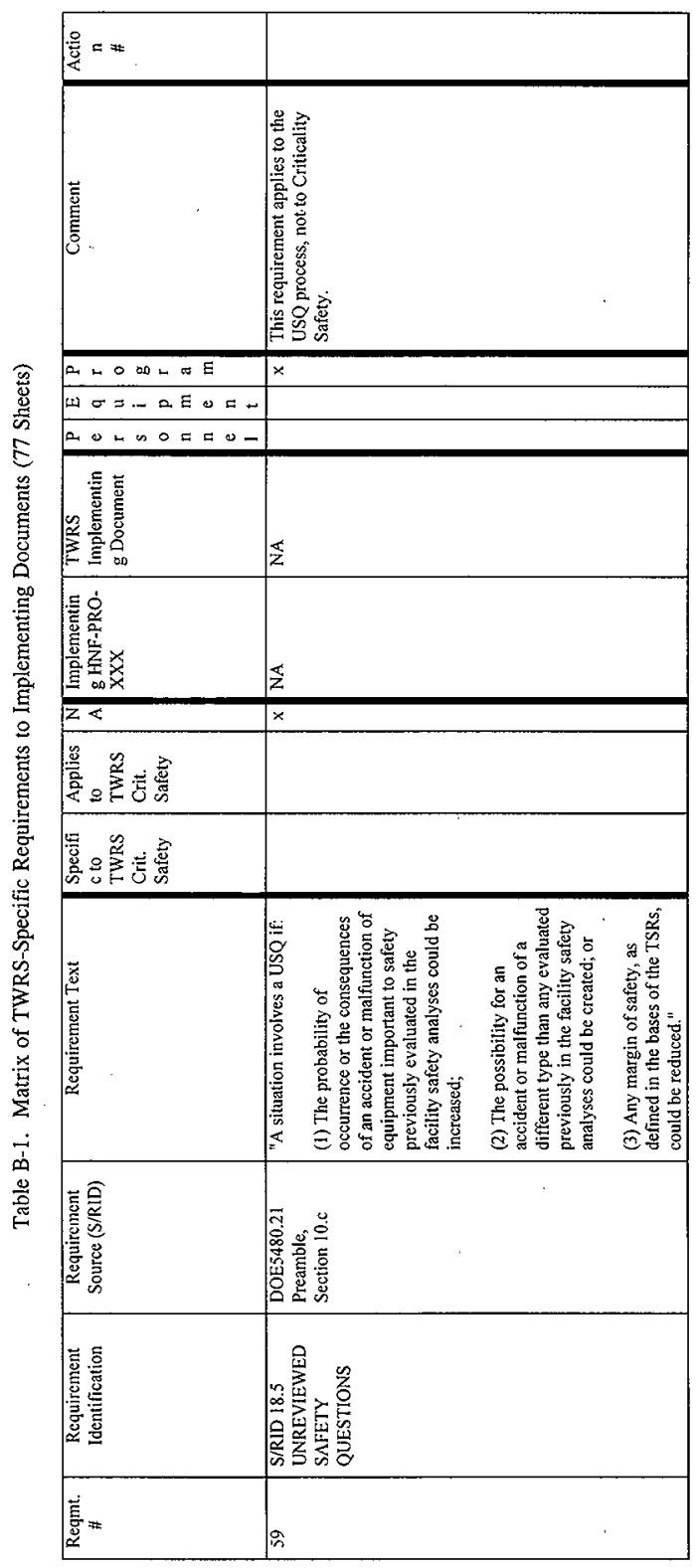

Page B-32 
TWR-3721 Rev. 0

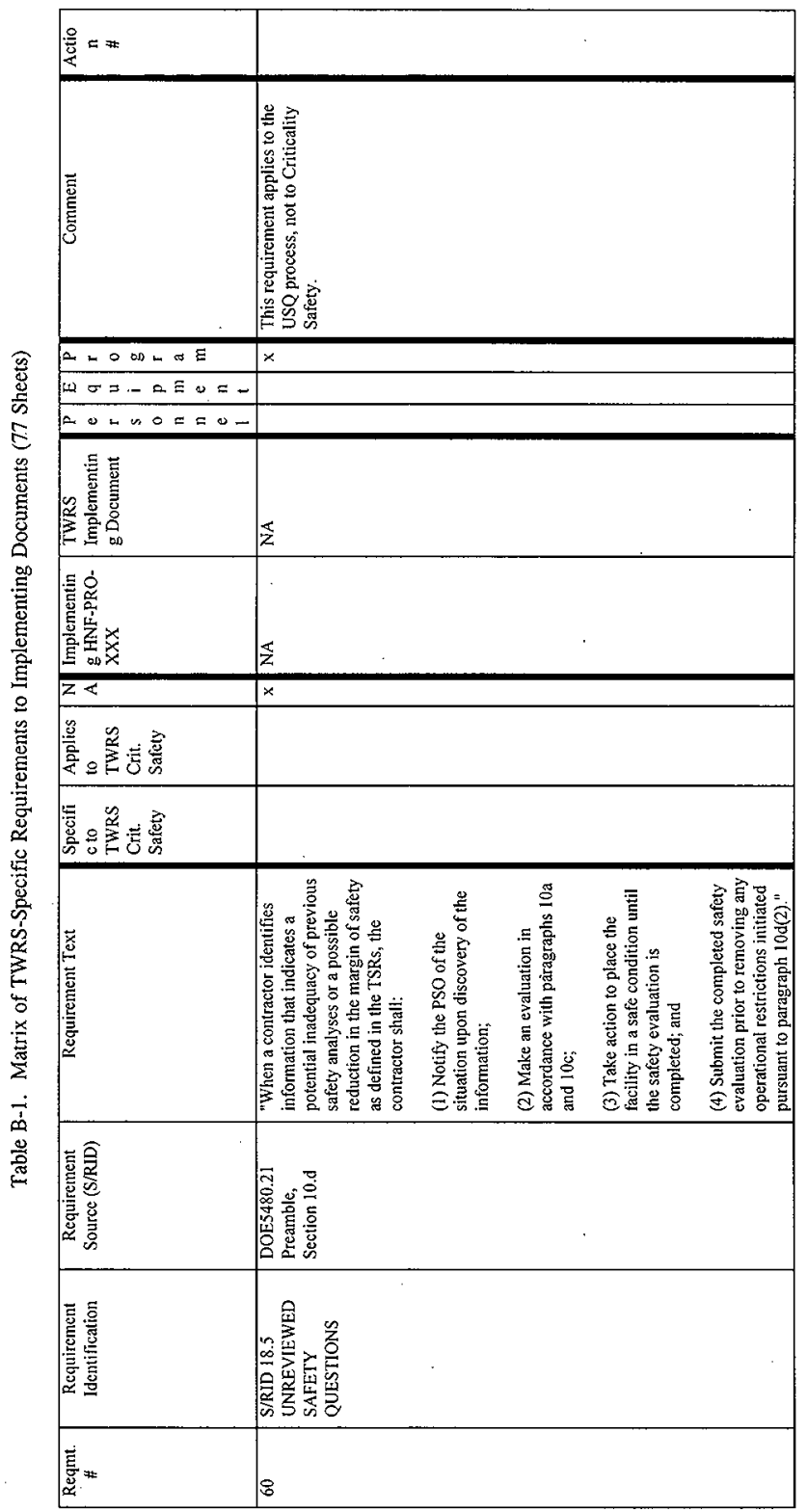

Page B-33 


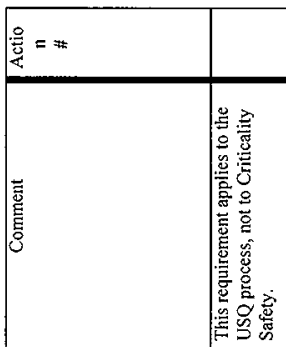

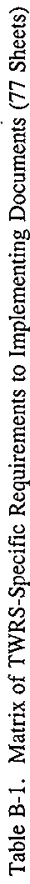

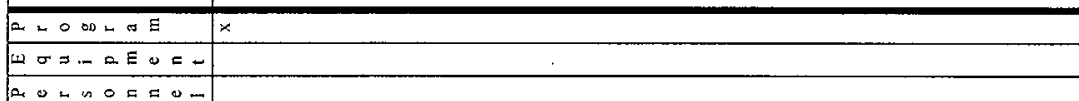

- Dot is OEFU-

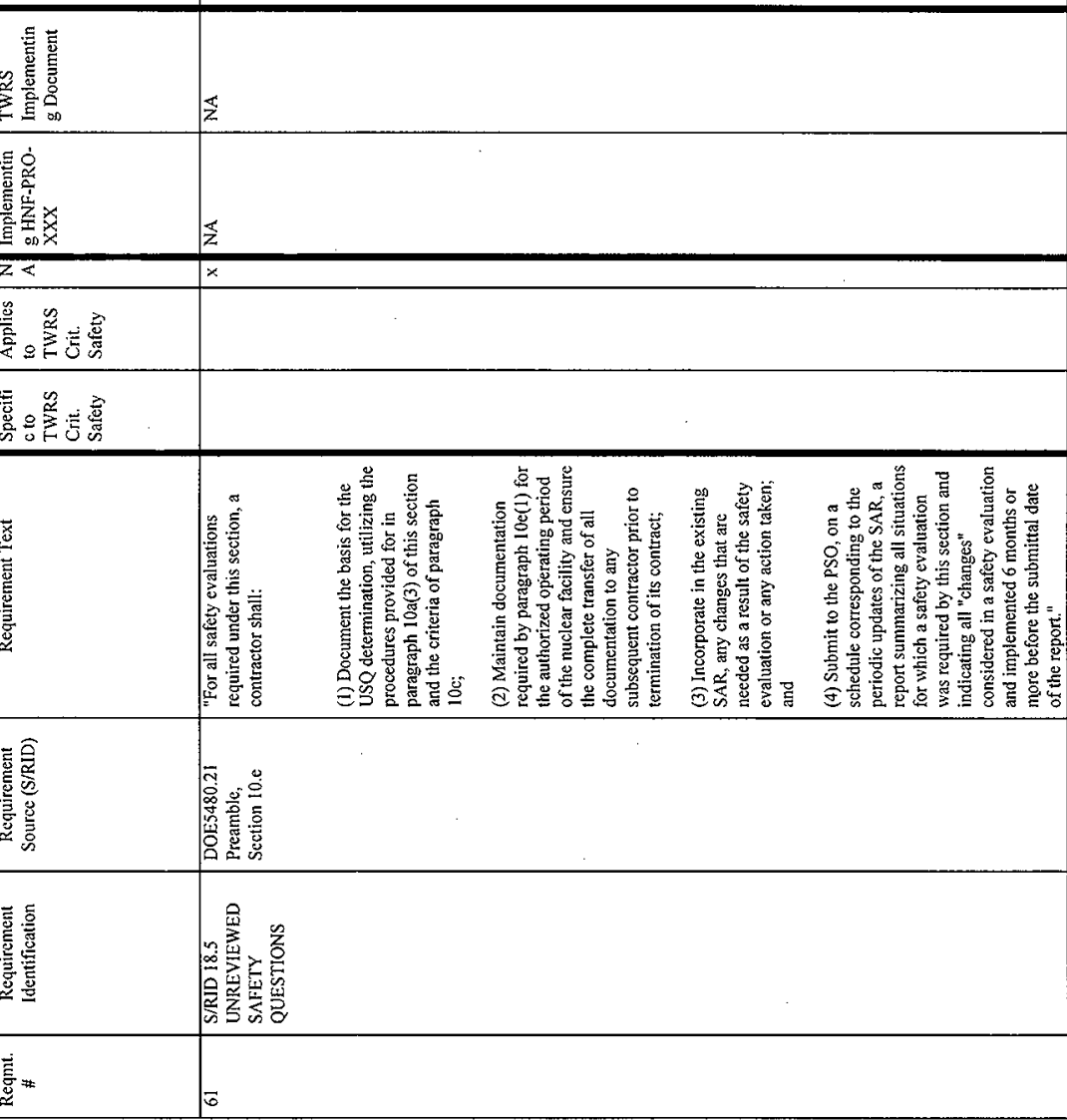


TWR-3721 Rev. 0

\begin{tabular}{|c|c|c|c|c|c|}
\hline$\frac{9}{4}=3$ & & & & & \\
\hline 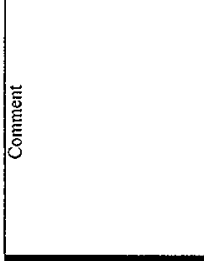 & 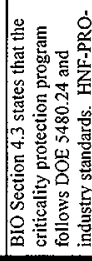 & 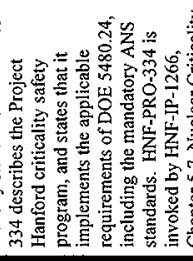 & 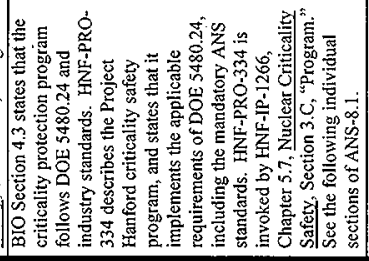 & & \\
\hline ALO OnL $L E$ & $x$ & & $x$ & $\%$ & $\star$ \\
\hline 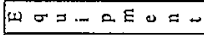 & & & $x$ & & \\
\hline $20-\infty 0==0-$ & & & $x$ & $x$ & 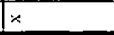 \\
\hline 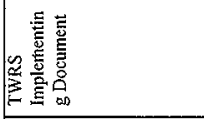 & 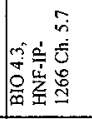 & & 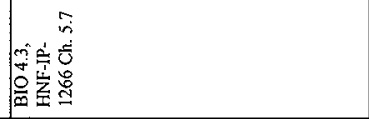 & 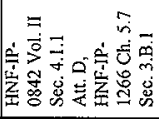 & 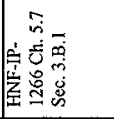 \\
\hline 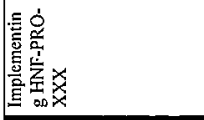 & 啹 & & ल & 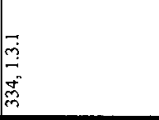 & \\
\hline & & & & & \\
\hline 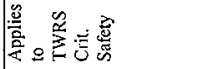 & & & . & & \\
\hline 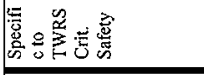 & $x$ & & $\times$ & $x$ & $\times$ \\
\hline 这 & 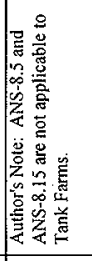 & 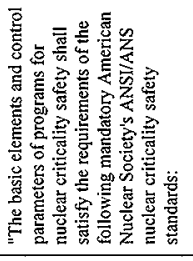 & 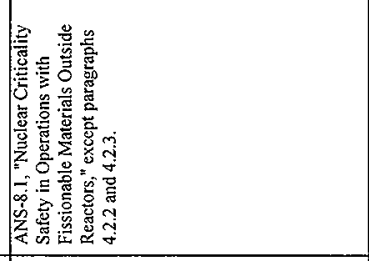 & 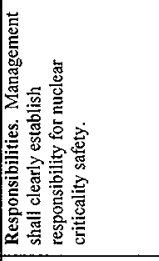 & 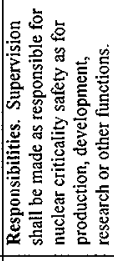 \\
\hline 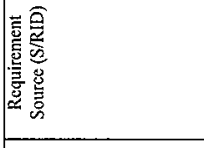 & 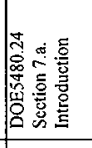 & & 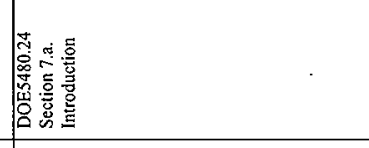 & & \\
\hline 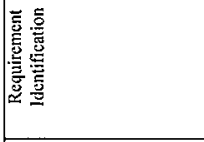 & 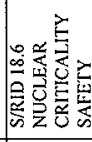 & & 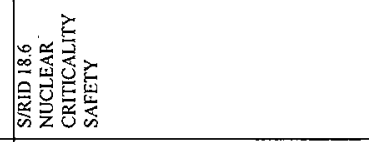 & 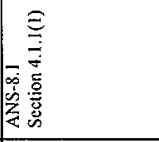 & 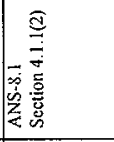 \\
\hline 言 & $\approx$ & & 8 & 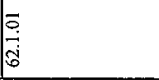 & 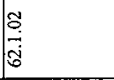 \\
\hline
\end{tabular}

Page B-35 
TWR-3721 Rev. 0

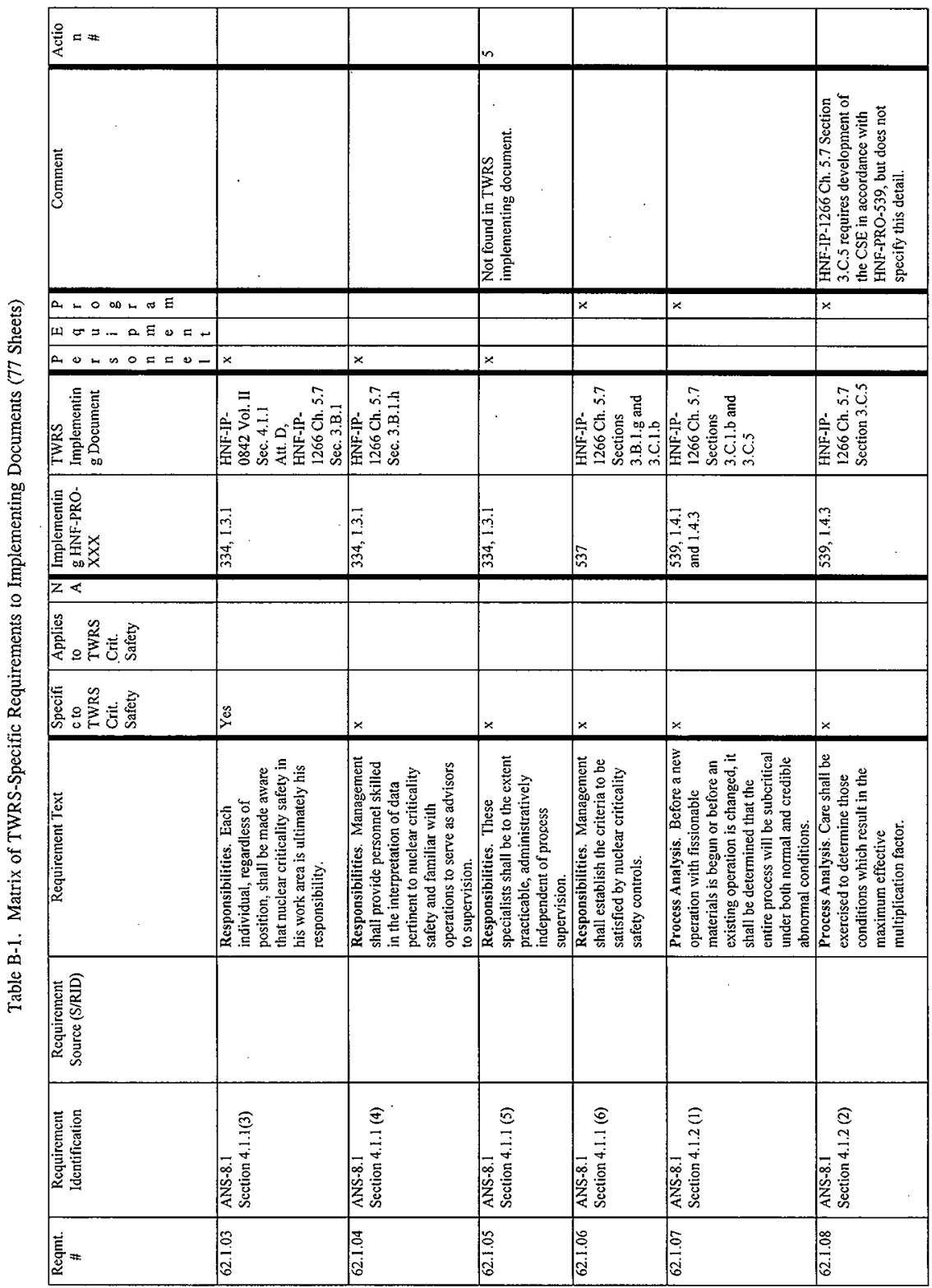




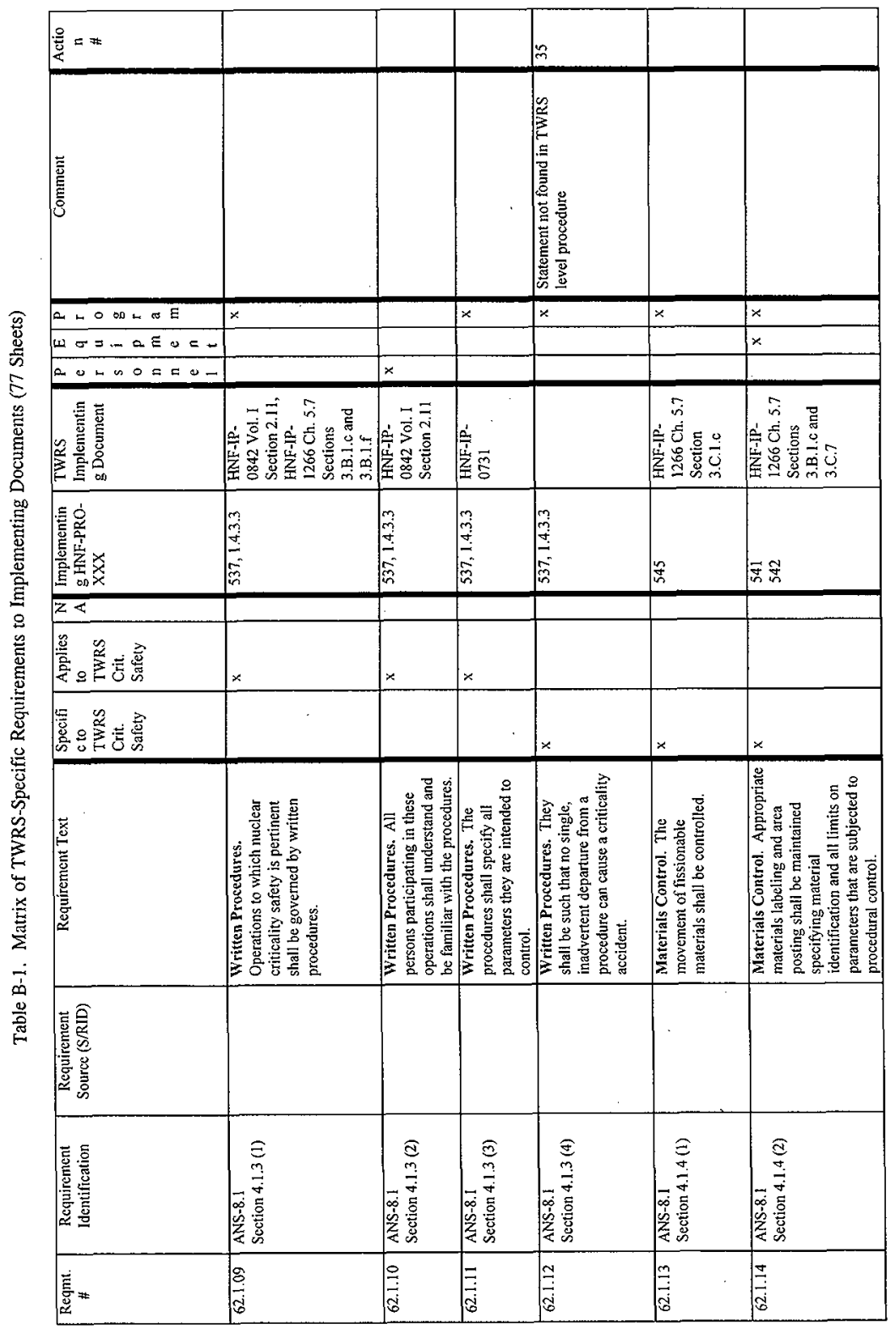

Page B-37 


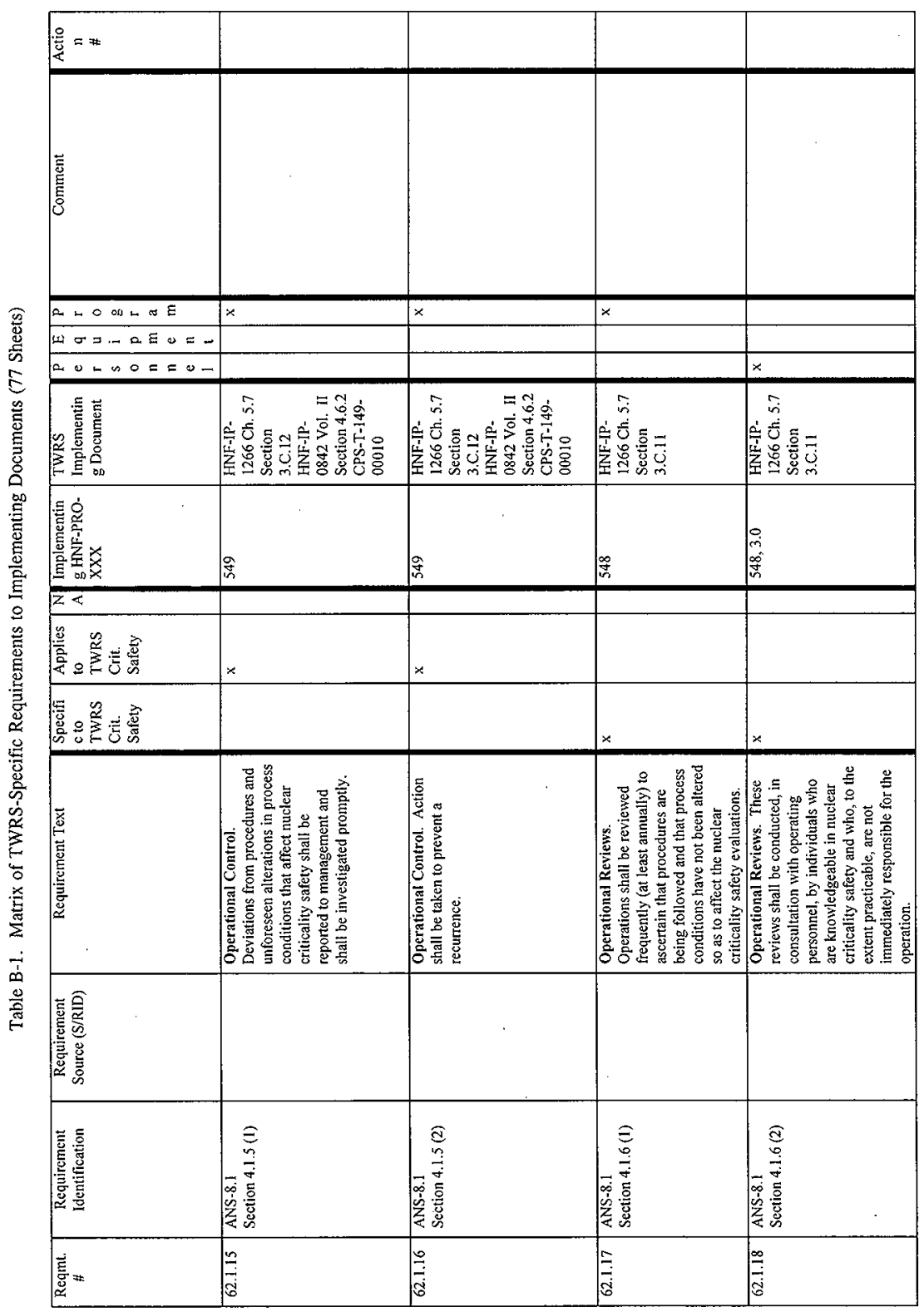


TWR-3721 Rev. 0

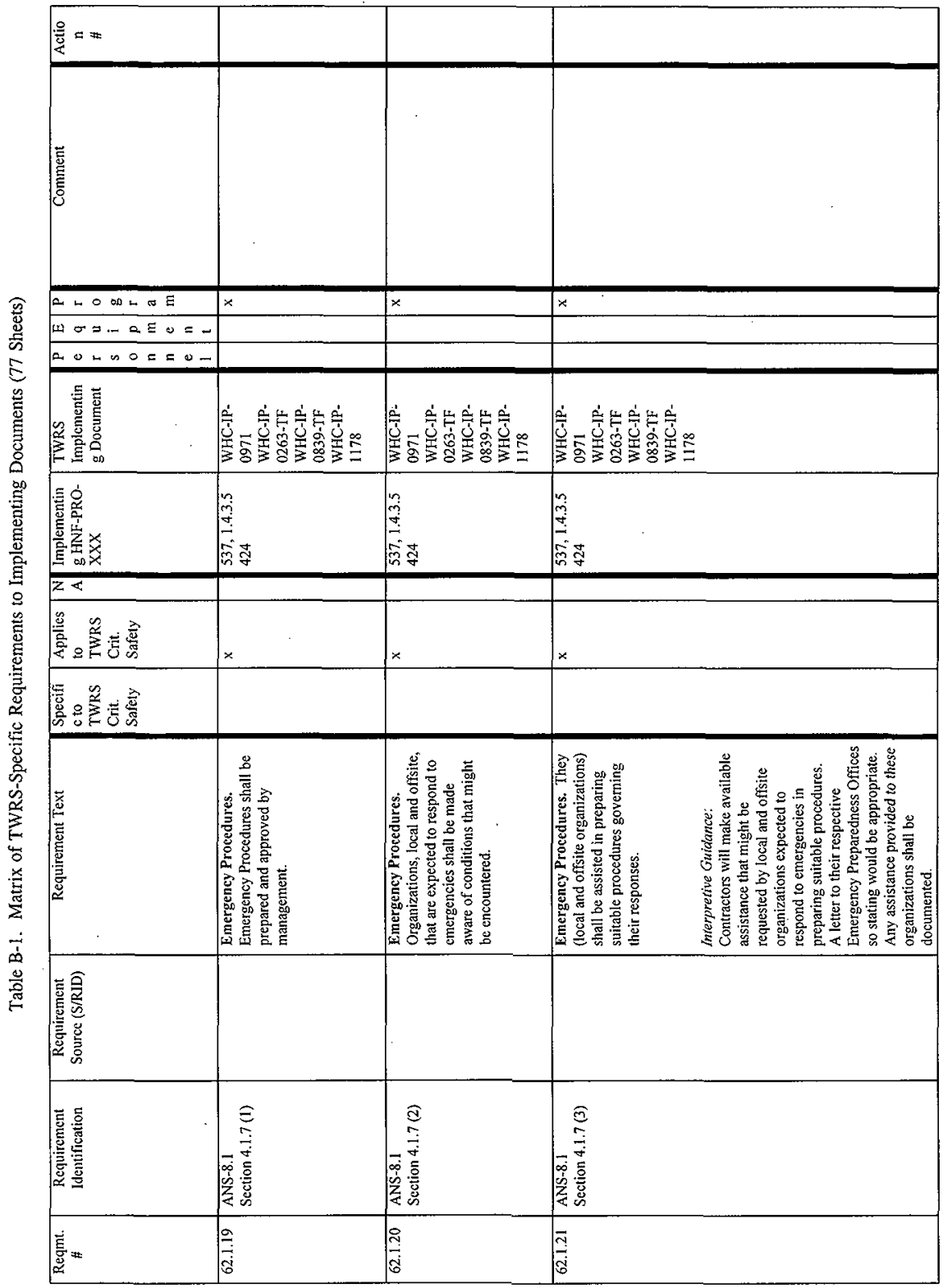

Page B-39 
TWR-3721 Rev. 0

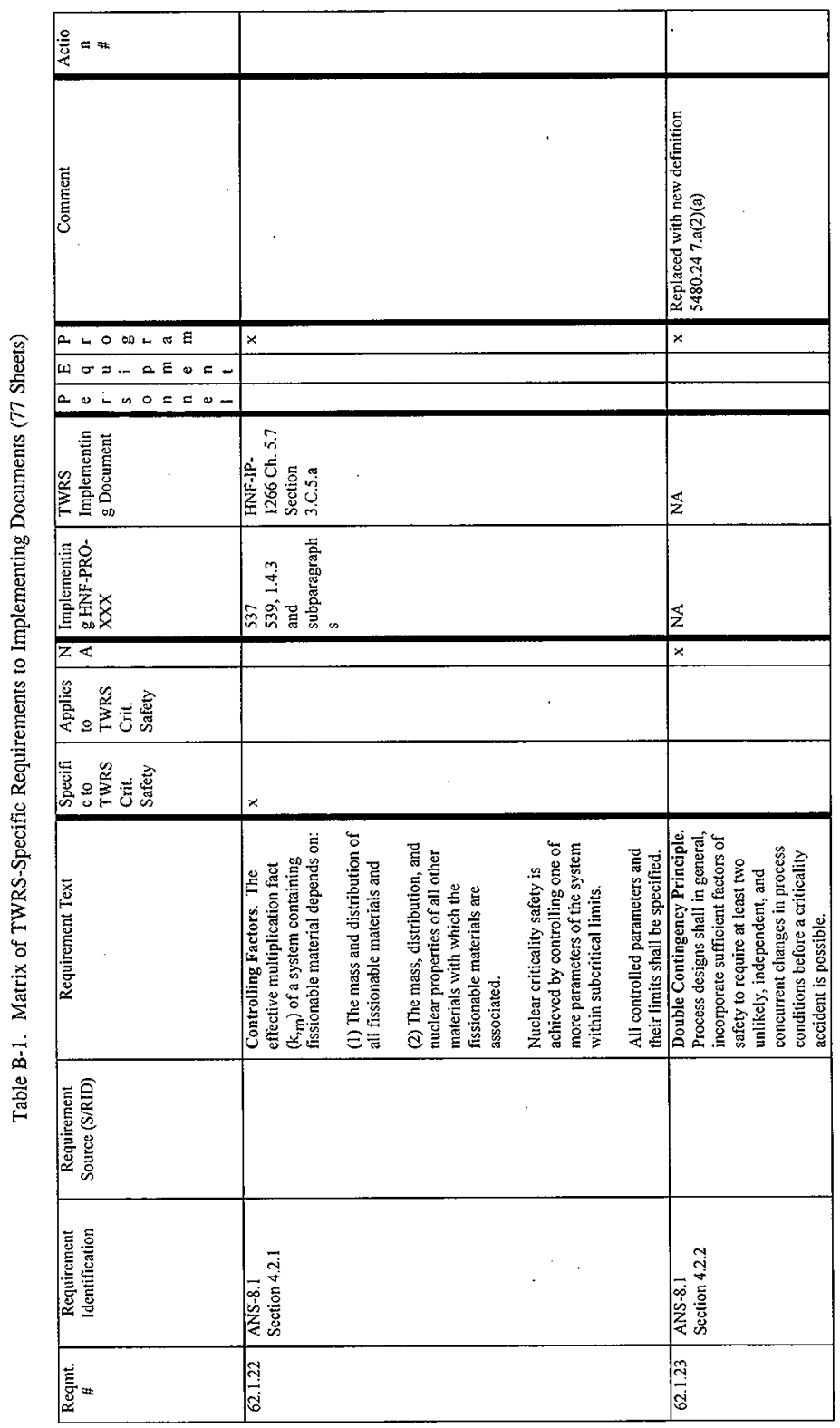

Page B- 40 
TWR-3721 Rev. 0

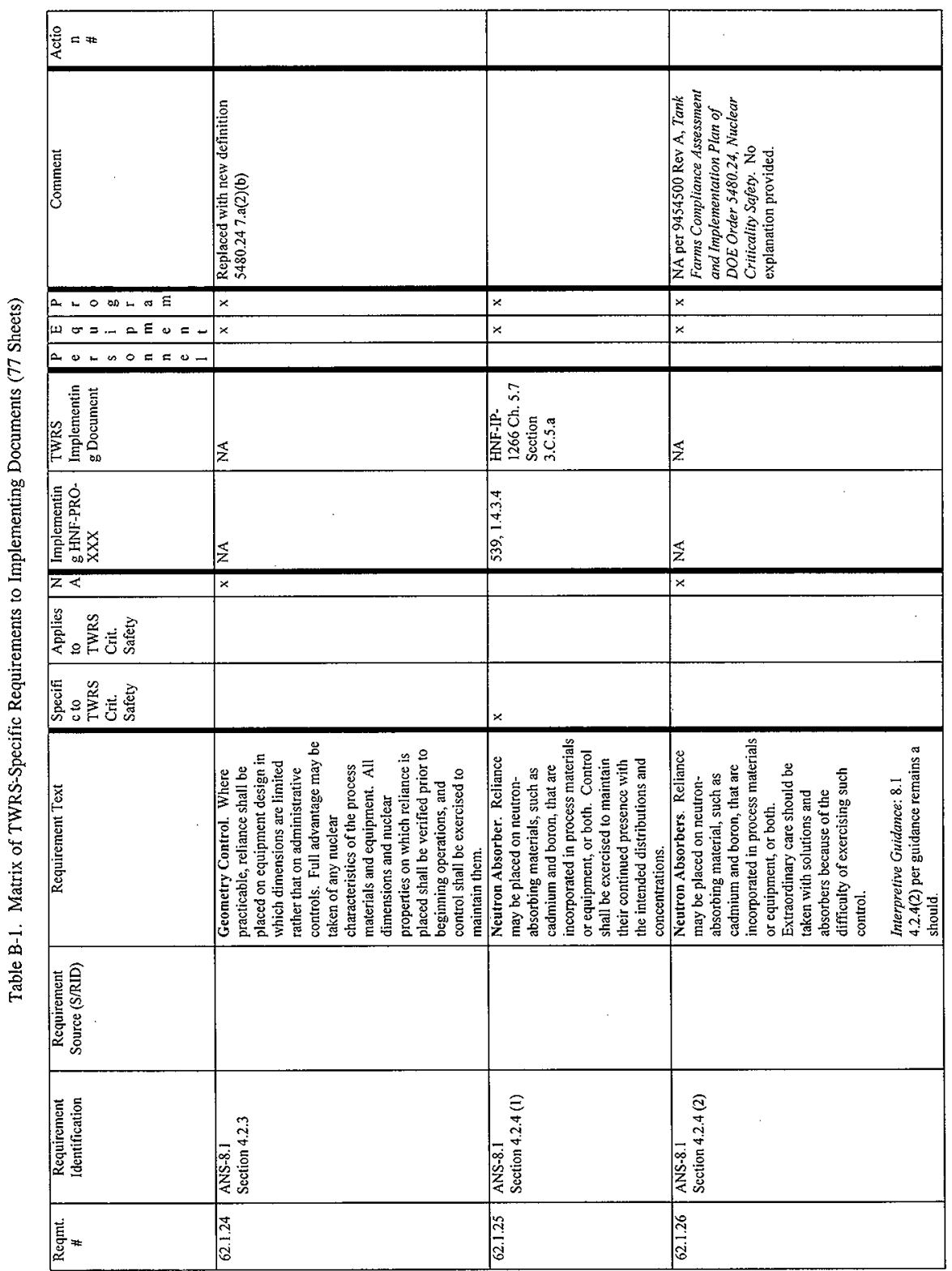




\begin{tabular}{|c|c|c|c|c|}
\hline$\frac{0}{2}=x$ & & & & \\
\hline | & & 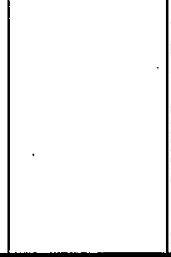 & 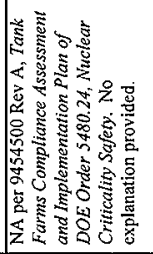 & \\
\hline$A-000=\sigma E$ & $\%$ & $x$ & $\approx$ & \\
\hline$\Leftrightarrow \sigma=\ldots \Omega \varepsilon=-$ & & & & \\
\hline $20+\infty O E=0$ & & & & \\
\hline 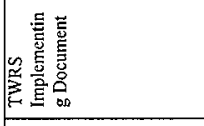 & 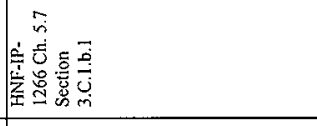 & 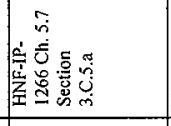 & $z$ & \\
\hline 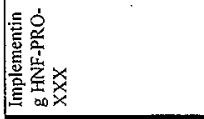 & 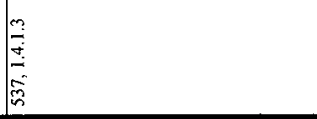 & 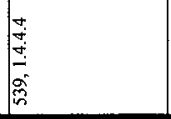 & $\frac{\pi}{2}$ & \\
\hline$z<$ & & & $x$ & \\
\hline 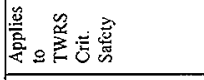 & & & & \\
\hline 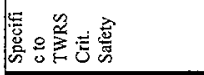 & $x$ & $x$ & & \\
\hline 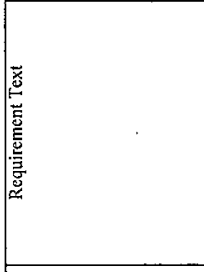 & 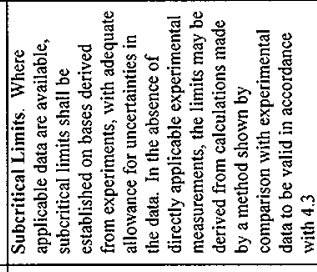 & 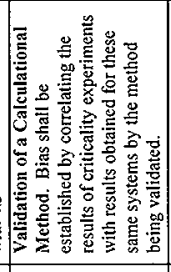 & 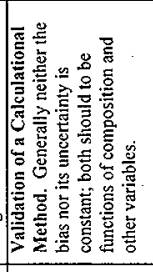 & 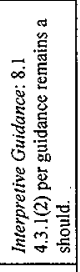 \\
\hline . & & & & \\
\hline 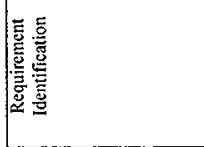 & 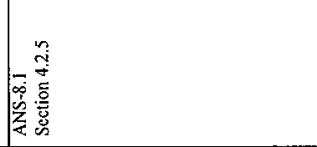 & 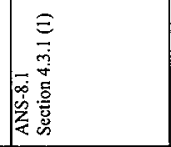 & 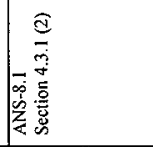 & \\
\hline 竧 & $\stackrel{\vec{j}}{\vec{N}}$ & نَ & 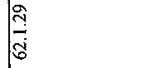 & \\
\hline
\end{tabular}


TWR-3721 Rev. 0

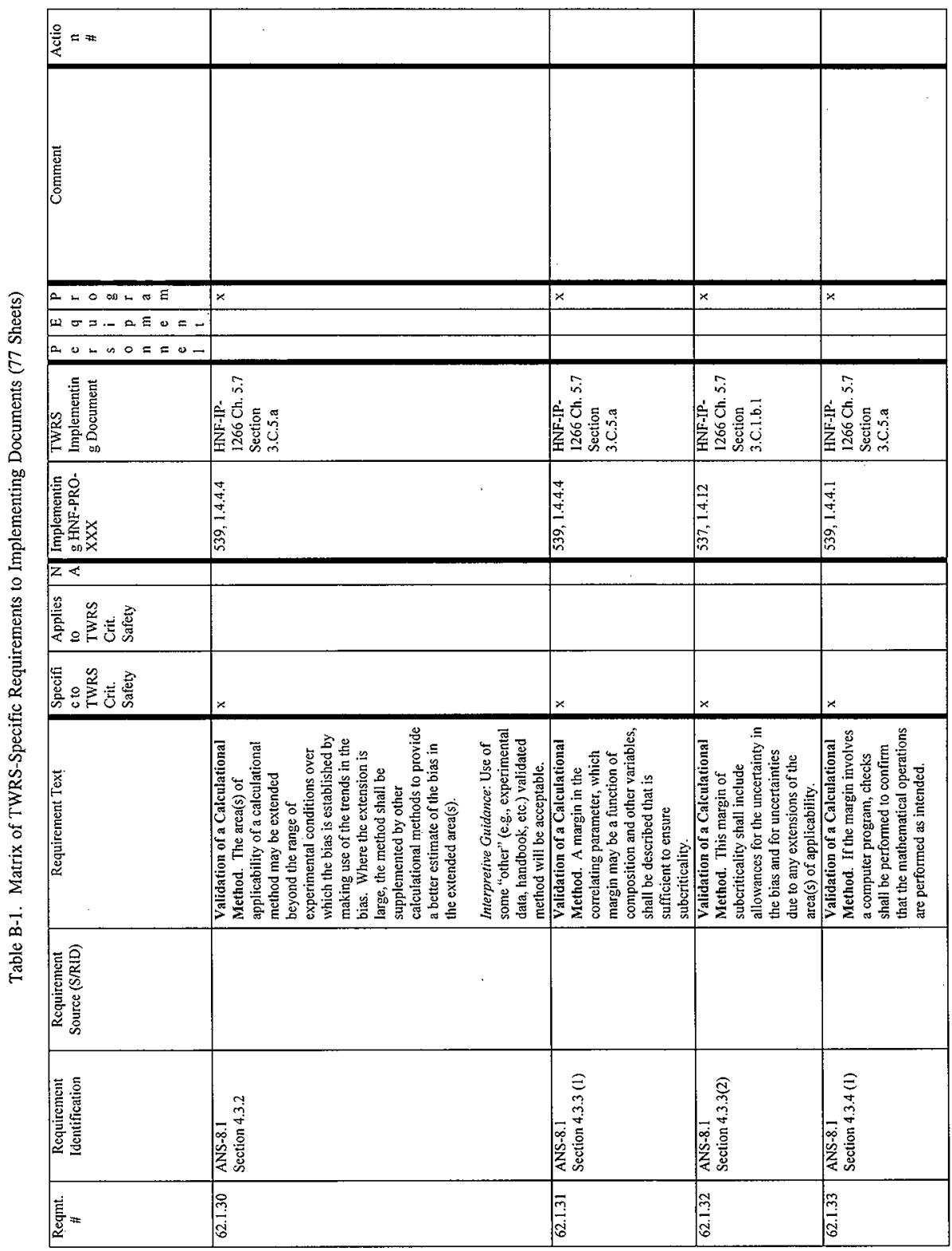


TWR-3721 Rev. 0

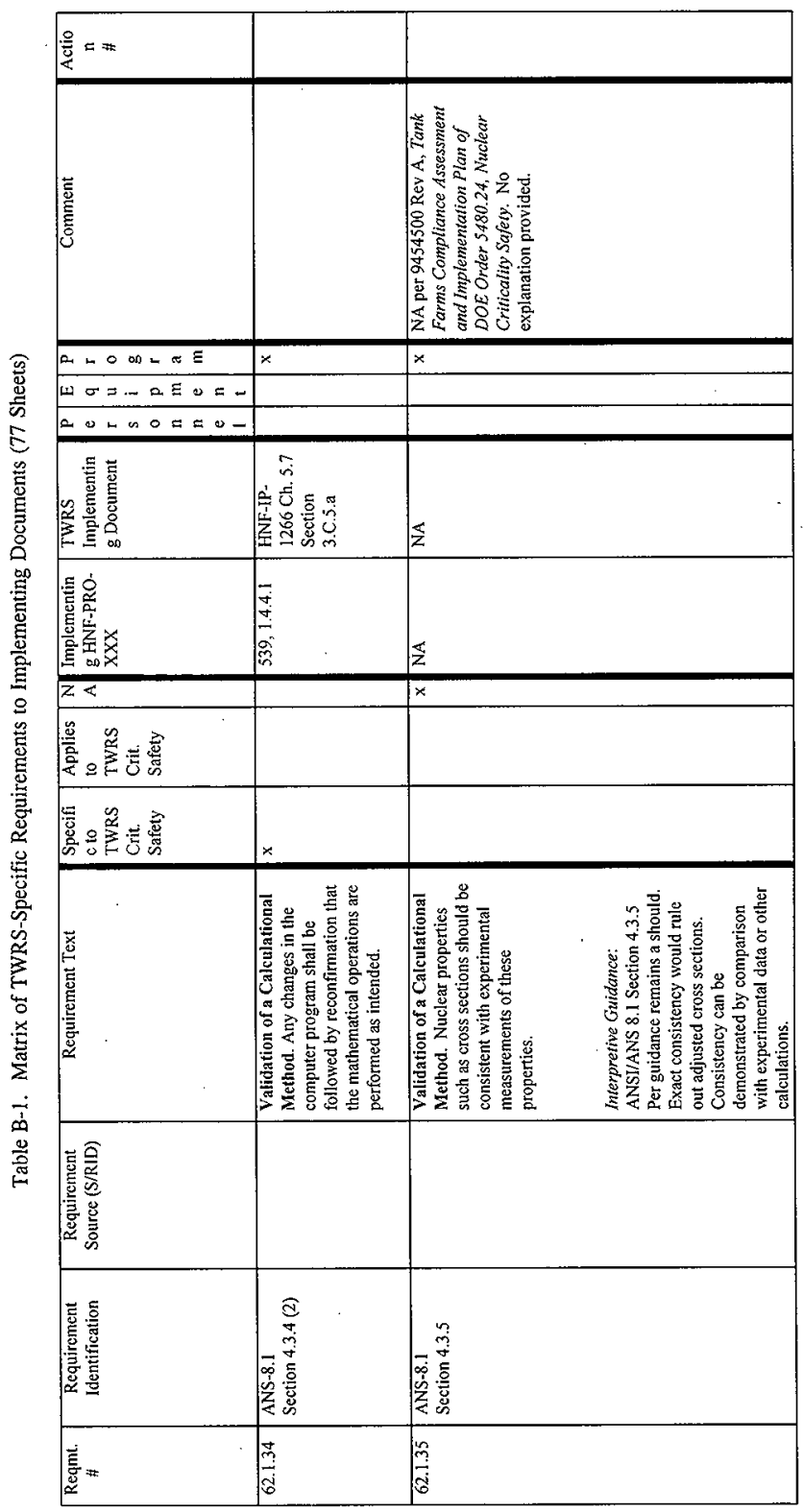

Page B-44 
TWR-3721 Rev. 0

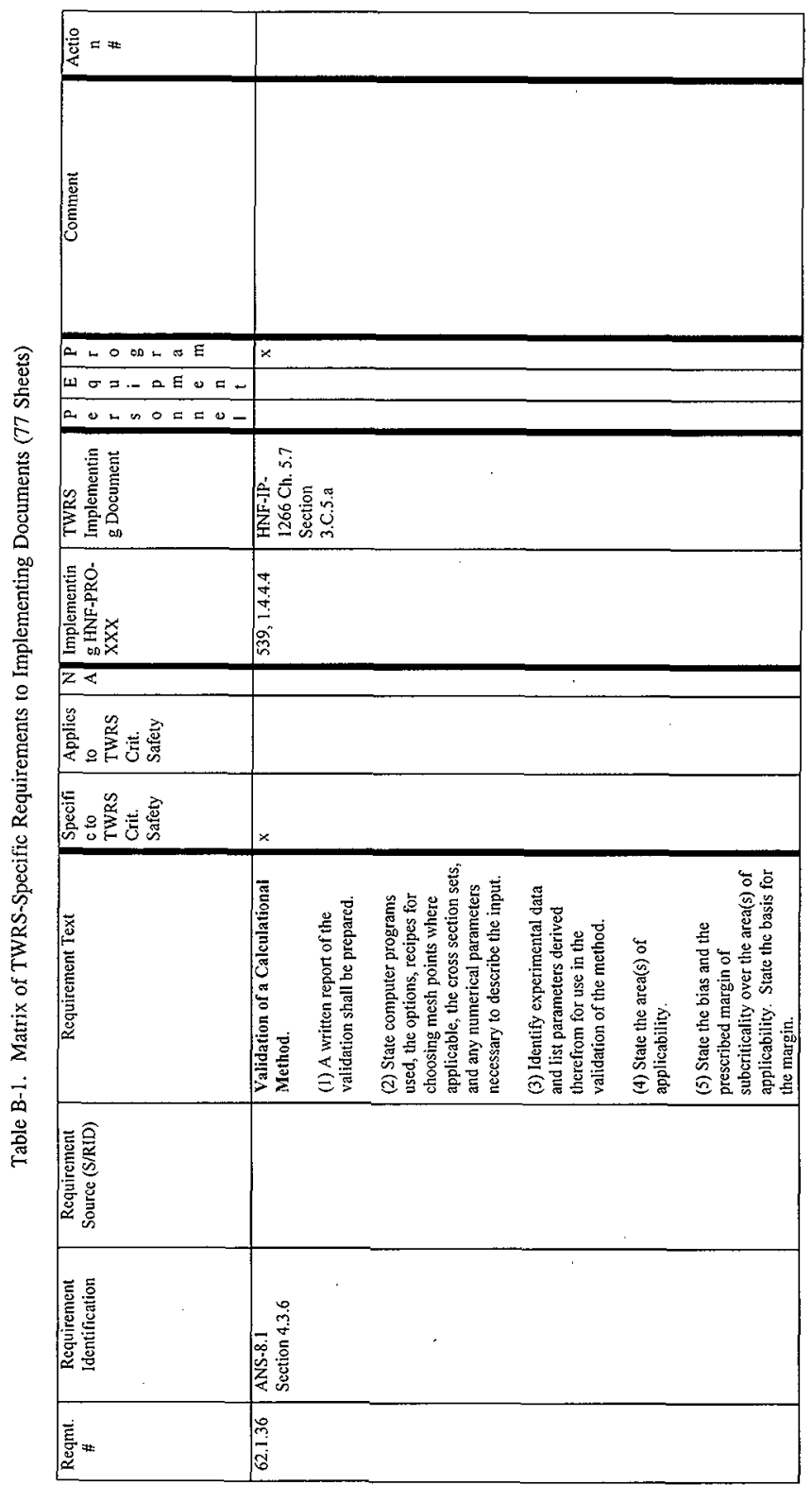

Page B-45 
TWR-3721 Rev. 0

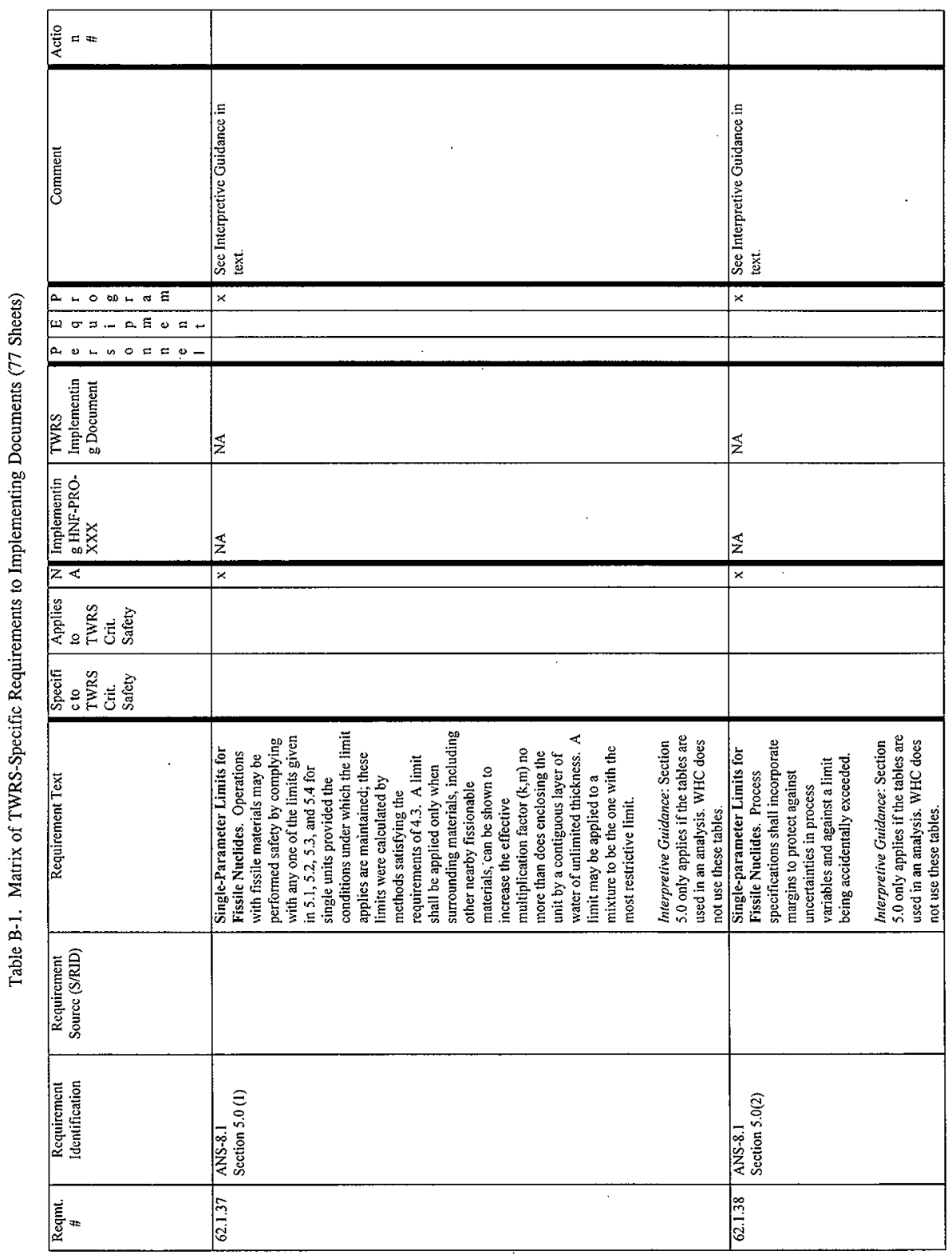


=

\begin{tabular}{|c|c|c|c|c|}
\hline 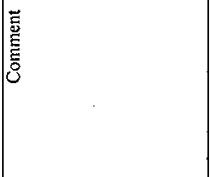 & 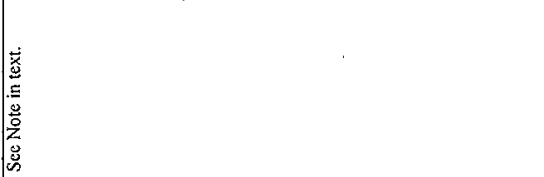 & & 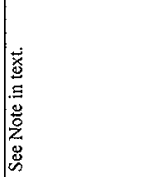 & \\
\hline 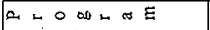 & $x$ & & $x$ & \\
\hline$\omega \sigma]-0 \equiv \omega=-$ & & & & \\
\hline notnog $\Rightarrow 0-$ & & & & \\
\hline 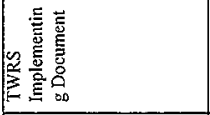 & $\frac{x}{z}$ & & $z$ & \\
\hline 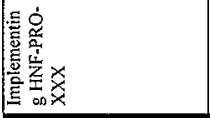 & $\frac{x}{z}$ & & $\alpha$ & \\
\hline$z<$ & $x$ & & $x$ & \\
\hline 总 & & & & \\
\hline 象密意 & ' & & & \\
\hline 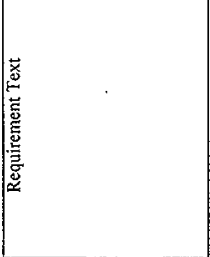 & 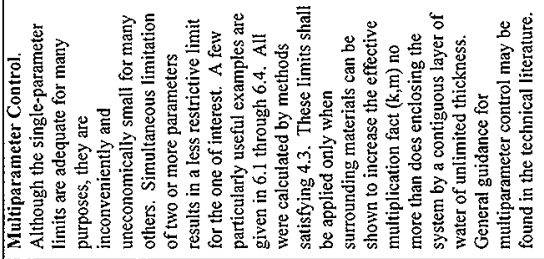 & 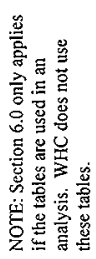 & 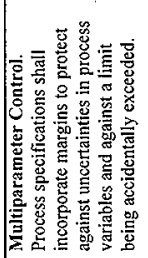 & 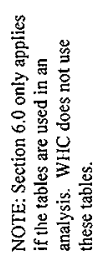 \\
\hline 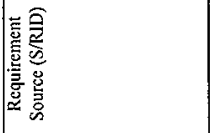 & & & & \\
\hline 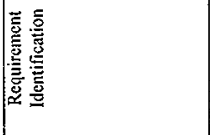 & 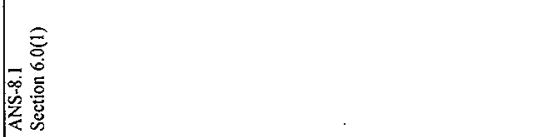 & & 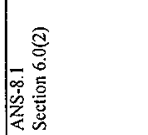 & \\
\hline 离 & 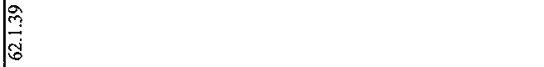 & & $\frac{8}{8}$ & \\
\hline
\end{tabular}




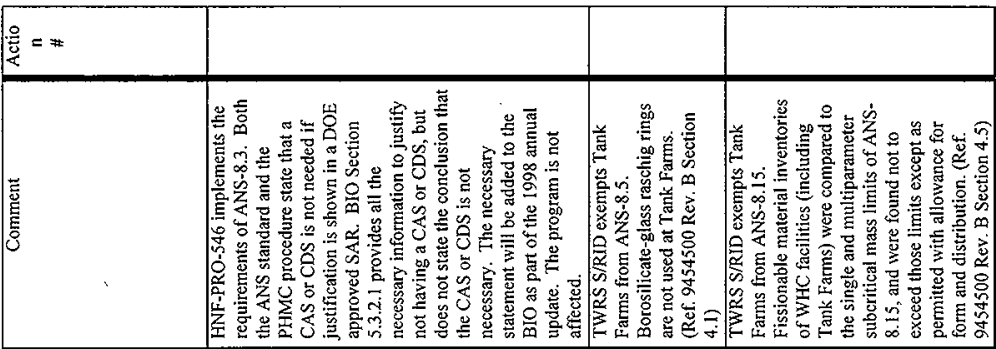

\begin{tabular}{|c|c|c|c|}
\hline AnO000 & $x$ & $x$ & $x$ \\
\hline$\Leftrightarrow \circ] \ldots \circ E 0=-$ & $\approx$ & & \\
\hline 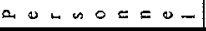 & & & \\
\hline 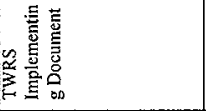 & 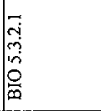 & 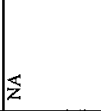 & $z$ \\
\hline 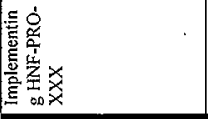 & 站 & $z$ & $z$ \\
\hline$z<$ & & $x$ & $x$ \\
\hline 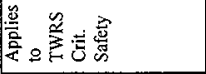 & & & \\
\hline 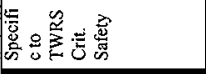 & $x$ & & \\
\hline 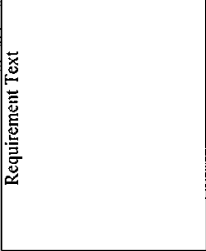 & 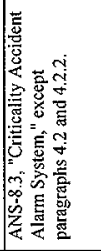 & 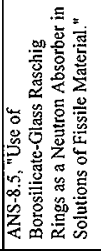 & 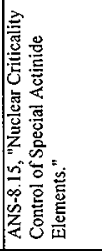 \\
\hline 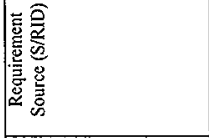 & 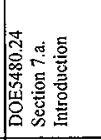 & 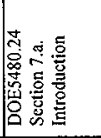 & 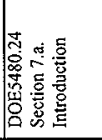 \\
\hline 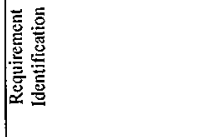 & 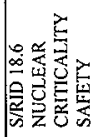 & 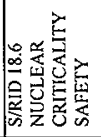 & 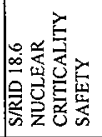 \\
\hline 壳 & $\widetilde{\widetilde{U}}$ & îj & 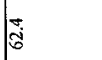 \\
\hline
\end{tabular}


TWR-3721 Rev. 0

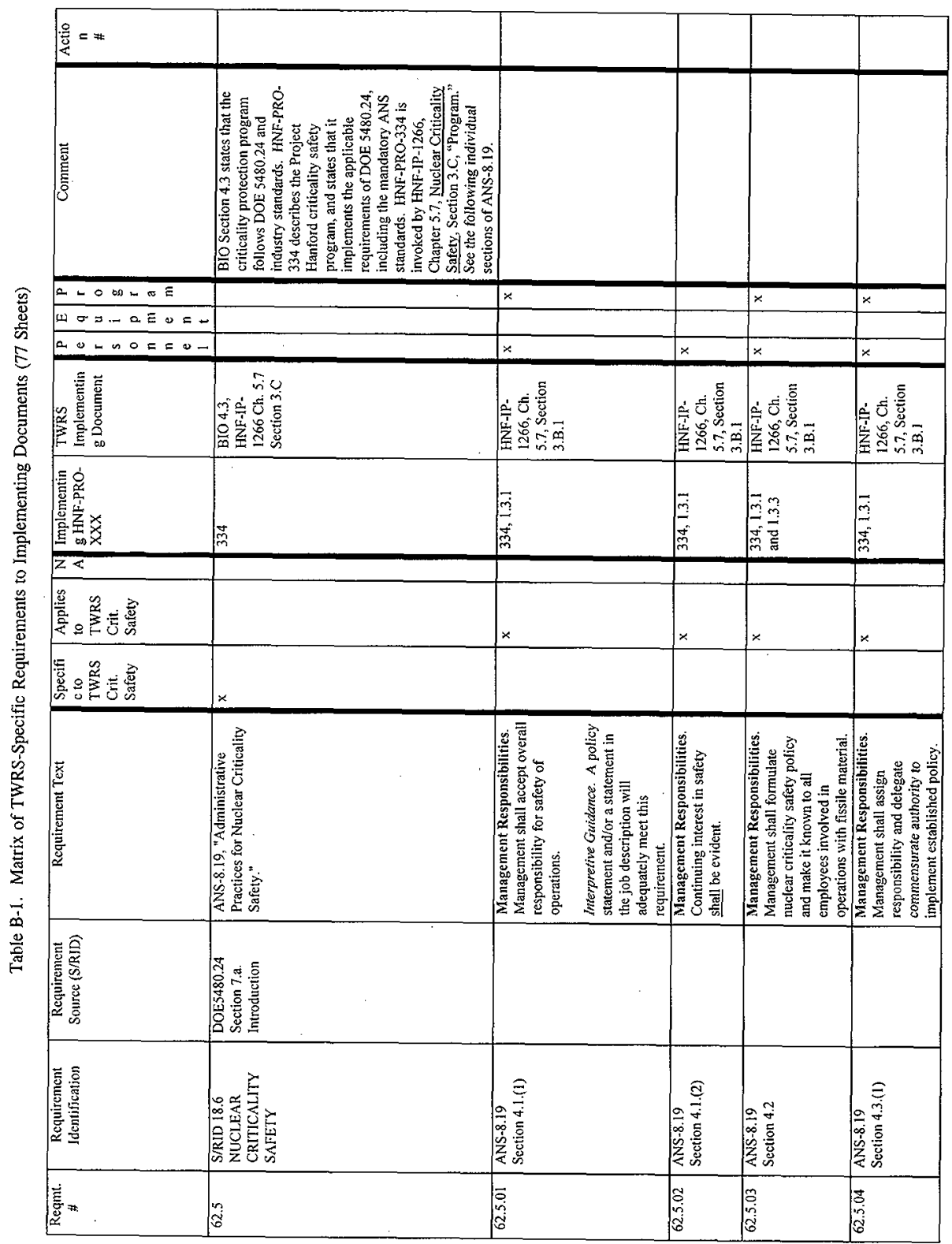

Page B-49 


\begin{tabular}{|c|c|c|c|c|c|c|c|}
\hline$=7$ & & & $\ln$ & & 禺 & & \\
\hline 言 & & & 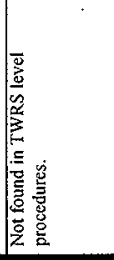 & & 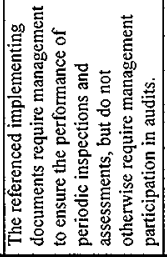 & & \\
\hline AnO OD口 E E & $\approx$ & & $x$ & $x$ & $x$ & & \\
\hline$\omega \sigma s-\alpha E \nu=-$ & & & & & & & \\
\hline $00 \ln 0=00-$ & & 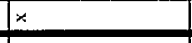 & $x$ & & & $x$ & $x$ \\
\hline 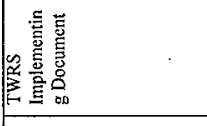 & 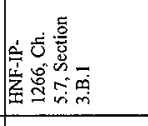 & 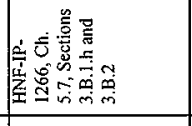 & 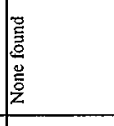 & 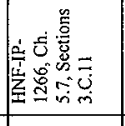 & 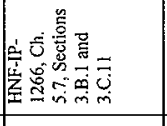 & 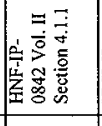 & 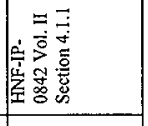 \\
\hline 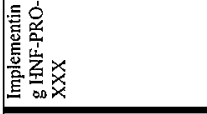 & 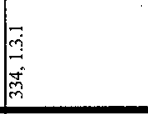 & 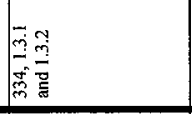 & $\begin{array}{l}\overrightarrow{3} \\
2 \\
\vec{m} \\
\vec{m}\end{array}$ & 慗 & $\mid \frac{8}{2}$ & & \\
\hline$z \varangle$ & & & & & & & \\
\hline 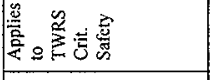 & & & & & & $x$ & \\
\hline 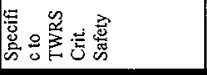 & $\times$ & $\times$ & $\times$ & $x$ & $\times$ & & $\times$ \\
\hline 㟥 & 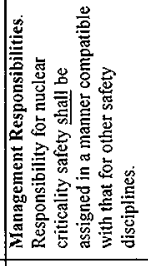 & 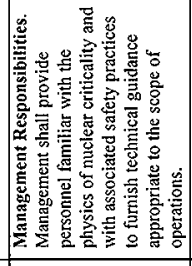 & 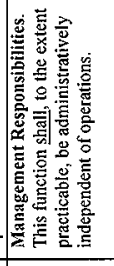 & 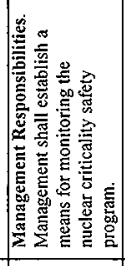 & 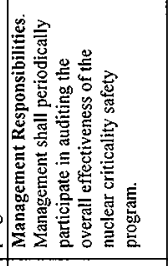 & 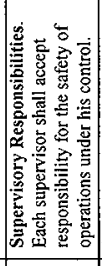 & 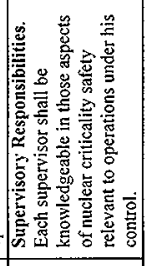 \\
\hline 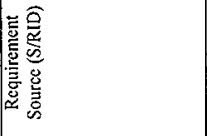 & & & & & & & \\
\hline 泀 & 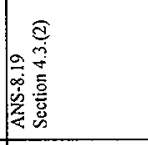 & 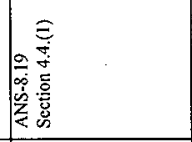 & 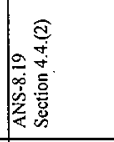 & 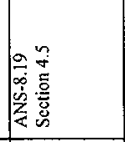 & 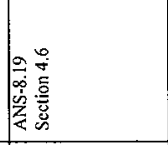 & 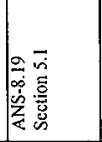 & 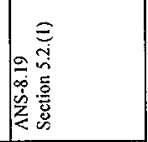 \\
\hline 言 & $\begin{array}{l}0 \\
0 \\
0 \\
0 \\
0\end{array}$ & 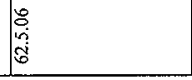 & \begin{tabular}{|l}
0 \\
0 \\
0 \\
0 \\
0 \\
\end{tabular} & $\begin{array}{l}\infty \\
0 \\
0 \\
0 \\
0 \\
0 \\
0\end{array}$ & $\begin{array}{l}0 \\
0 \\
0 \\
0 \\
0\end{array}$ & 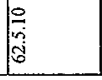 & $\begin{array}{l}\overline{0} \\
0 \\
0\end{array}$ \\
\hline
\end{tabular}




\begin{tabular}{|c|c|c|c|c|c|c|}
\hline$\frac{9}{3}=*$ & & & & & & n \\
\hline 吾 & & & & & & 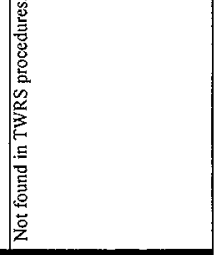 \\
\hline A-O off $n$ of $E$ & & & $x$ & $\approx$ & $x$ & \\
\hline$\Theta \sigma a \ldots 2 \equiv 0=+$ & & & & & & $x$ \\
\hline $00+\infty 0=20$ & $x$ & $x$ & & $\#$ & $x$ & $x$ \\
\hline 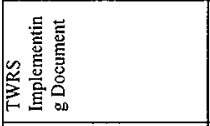 & 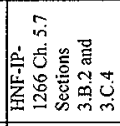 & 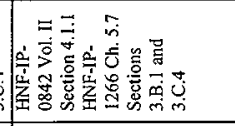 & 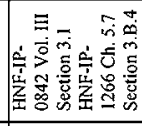 & 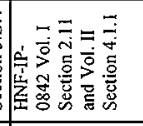 & 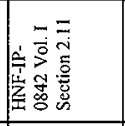 & \\
\hline 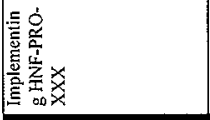 & 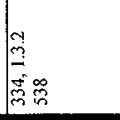 & 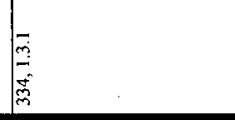 & 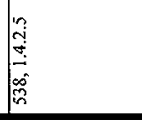 & & 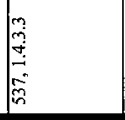 & 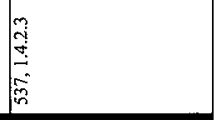 \\
\hline$z<$ & & & & & & \\
\hline 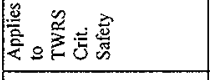 & & $x$ & $\times$ & $*$ & $x$ & $x$ \\
\hline 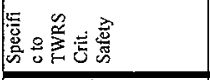 & $\times$ & & & & & \\
\hline 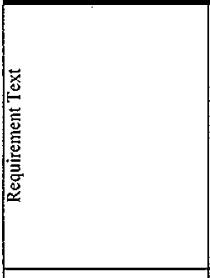 & 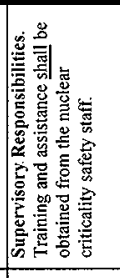 & 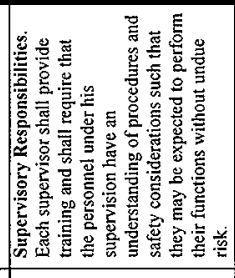 & 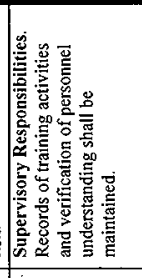 & 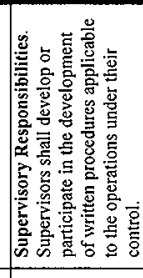 & 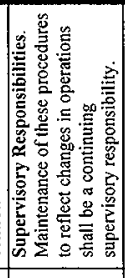 & 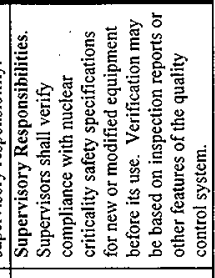 \\
\hline 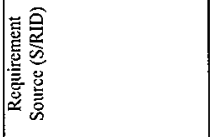 & & & & & & \\
\hline 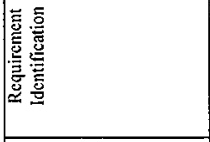 & 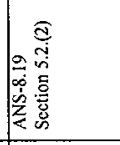 & 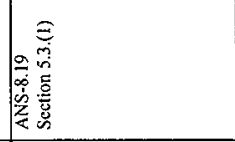 & 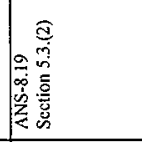 & 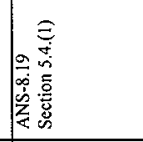 & 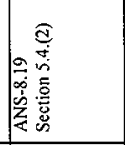 & 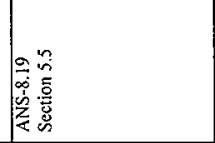 \\
\hline 童 & 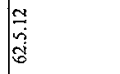 & $\underset{m}{m}$ & 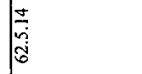 & 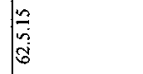 & $\mid$\begin{tabular}{l}
0 \\
\hdashline \\
\hdashline \\
0 \\
6
\end{tabular} & $=$ \\
\hline
\end{tabular}




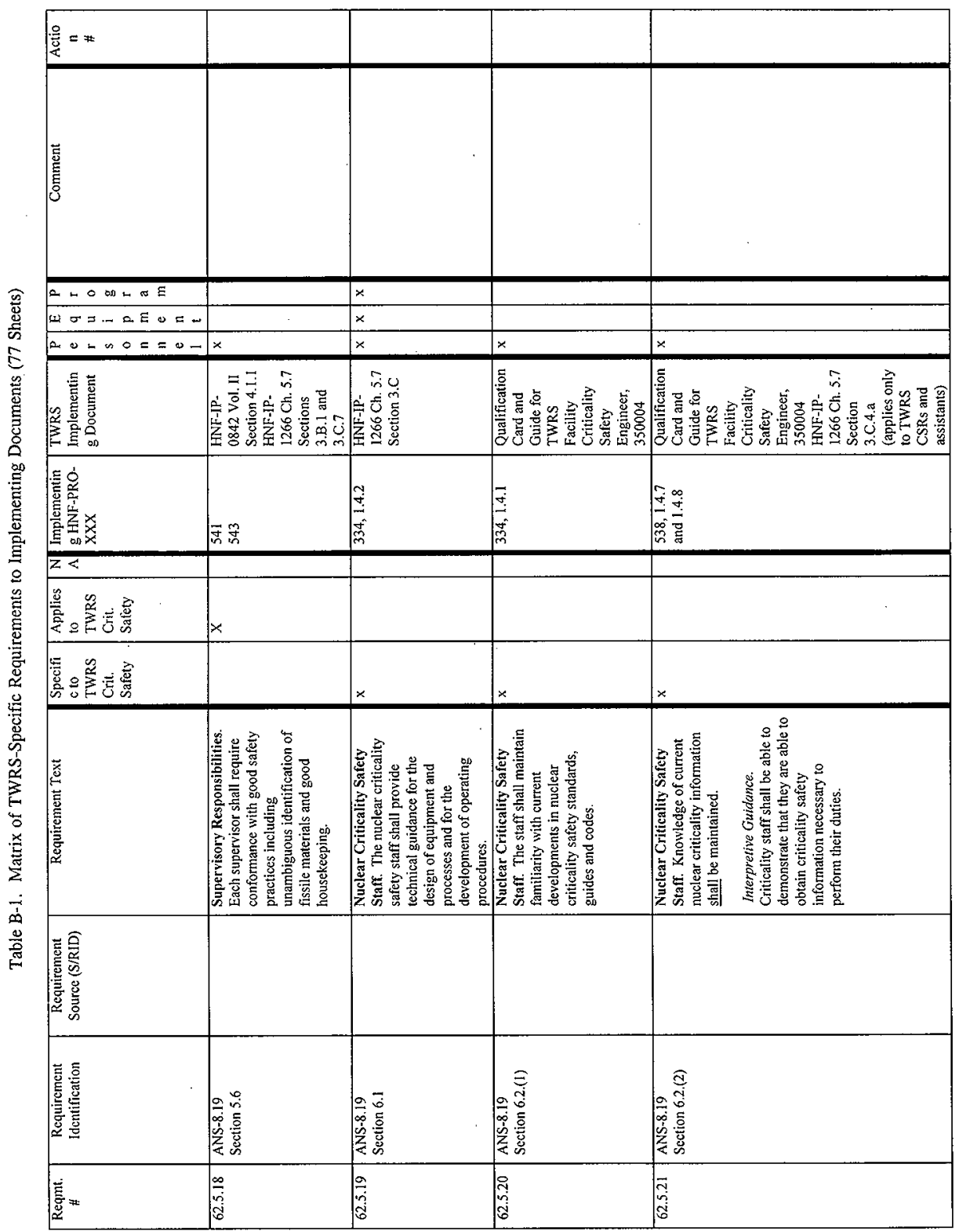


$\frac{0}{0}=$

D. O ODนUE

以

a. 0 - 0 a $a-x$

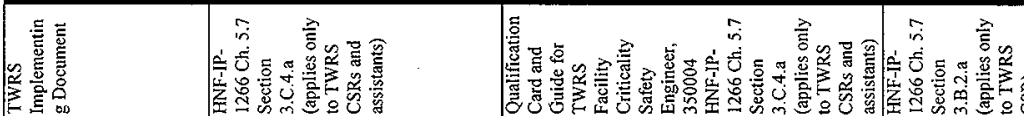

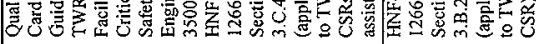

尊是

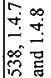

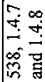

is

量

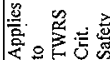

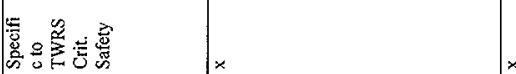

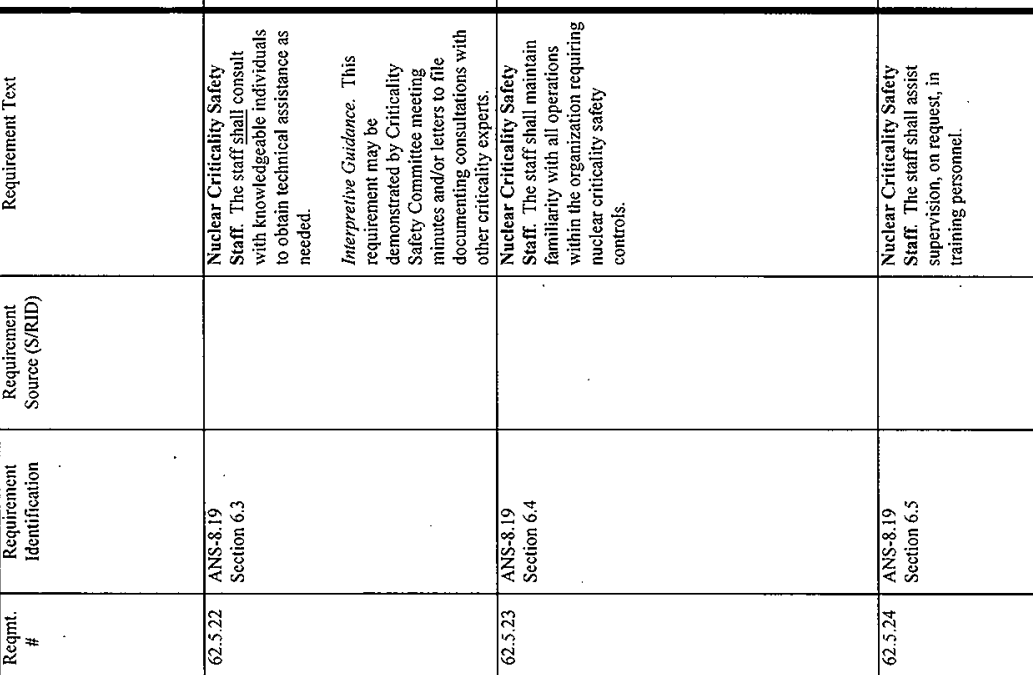

Page B-53 
TWR-3721 Rev. 0

各
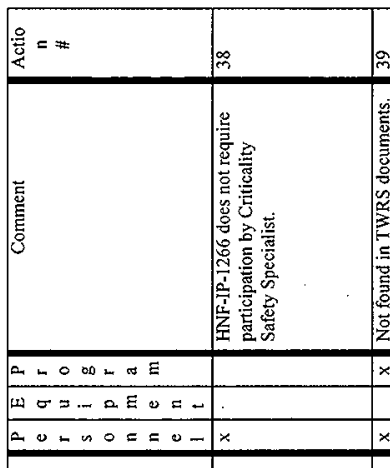

\begin{tabular}{|c|c|c|}
\hline 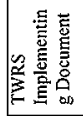 & 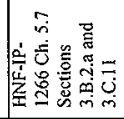 & \\
\hline 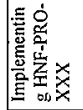 & 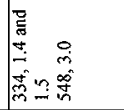 & $\begin{array}{l}m \\
\dot{m} \\
m \\
m\end{array}$ \\
\hline
\end{tabular}

훙

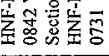

定홍

풍

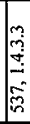

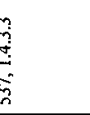

商

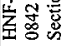

\begin{tabular}{|c|c|c|c|c|c|c|}
\hline$<$ & & & & & & \\
\hline 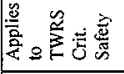 & & & $x$ & & $x$ & $x$ \\
\hline 递密离 & $x$ & $x$ & & & & \\
\hline 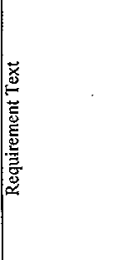 & 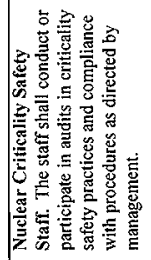 & 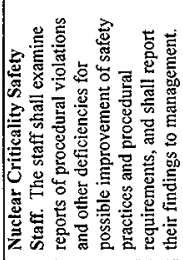 & 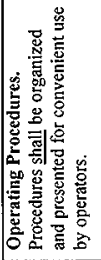 & 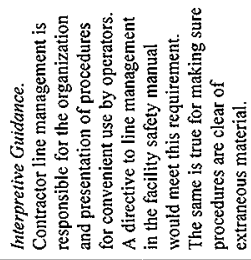 & 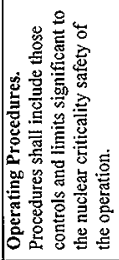 & 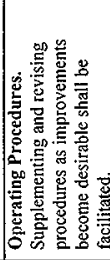 \\
\hline 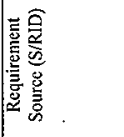 & & & & & & \\
\hline 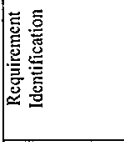 & 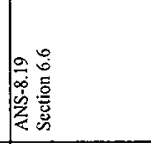 & 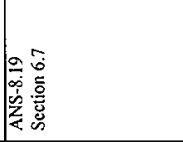 & 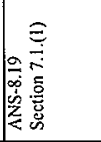 & & 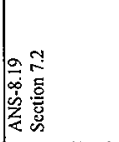 & 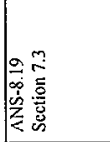 \\
\hline$\ddot{\Xi}_{\vec{E}}$ & 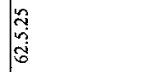 & 胥 & 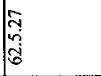 & & 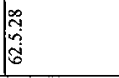 & 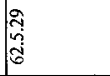 \\
\hline
\end{tabular}




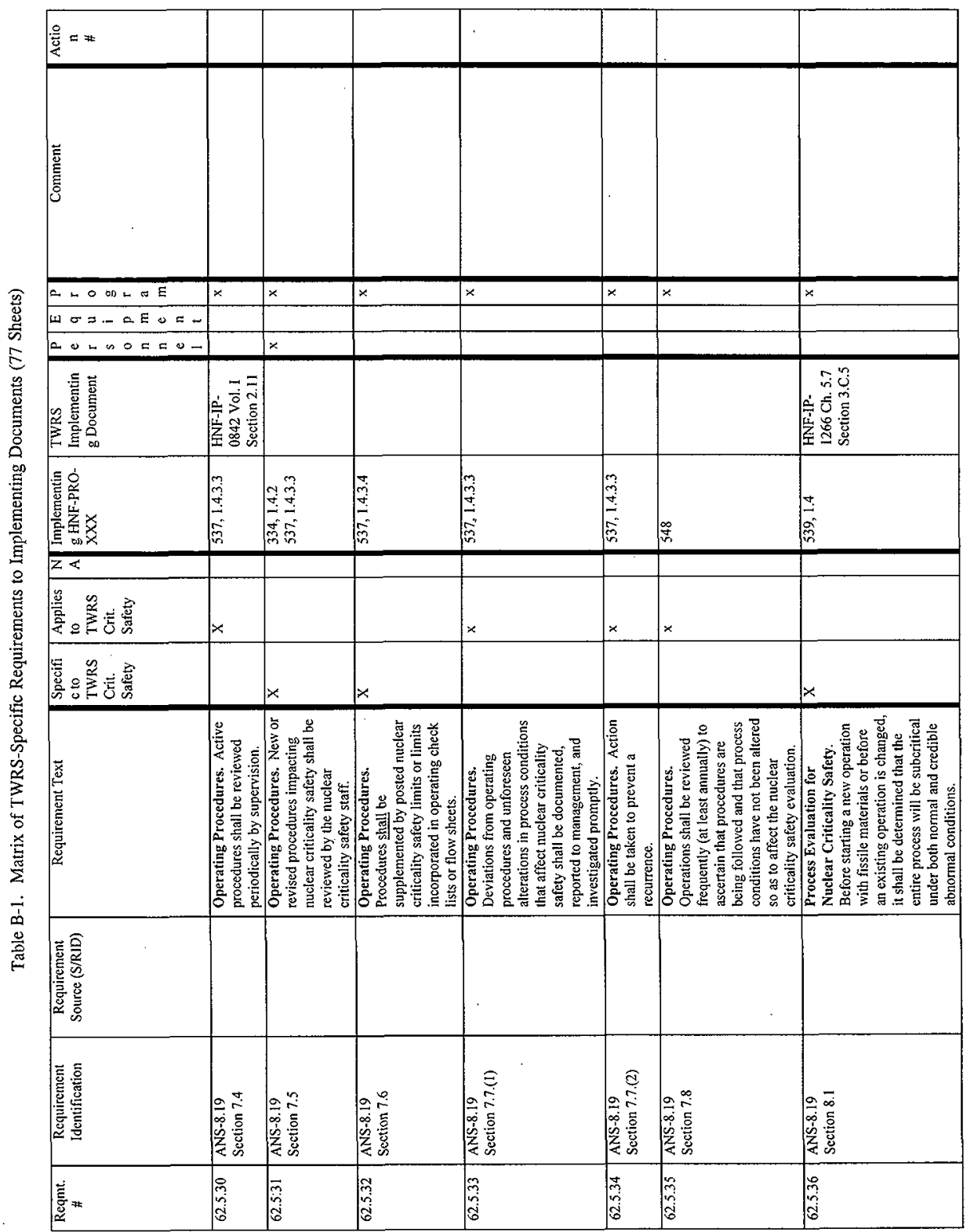




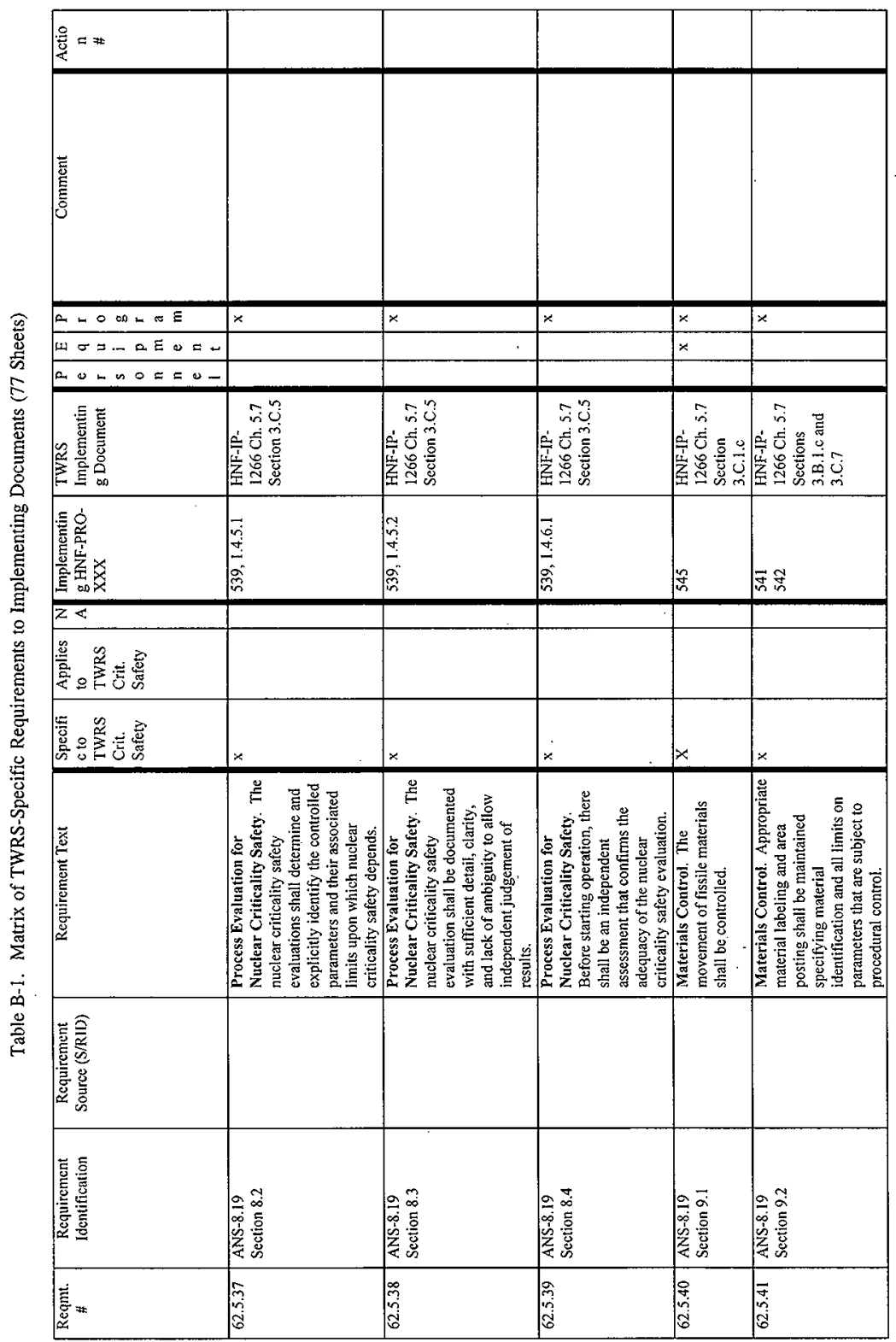


TWR-3721 Rev. 0

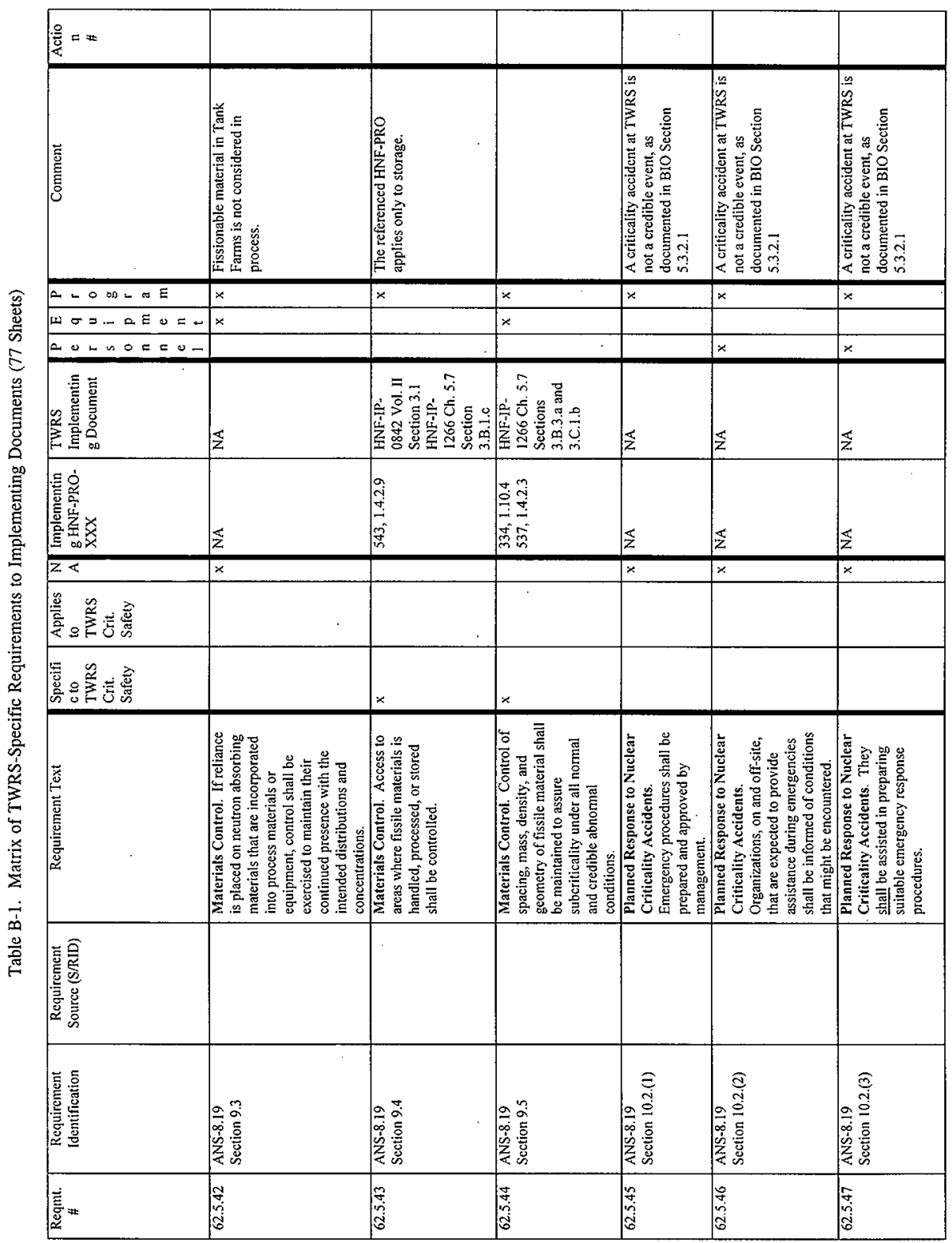


TWR-3721 Rev, 0

\begin{tabular}{|c|c|c|c|c|c|c|c|}
\hline 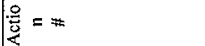 & & & & & & & \\
\hline 泀 & 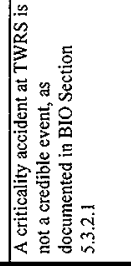 & & 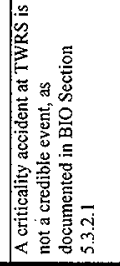 & 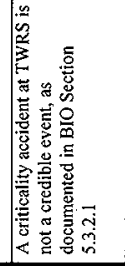 & 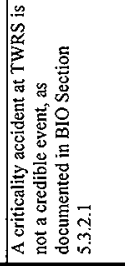 & 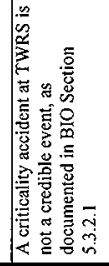 & 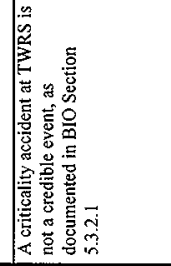 \\
\hline$A \leftarrow \circ 00 \backsim \approx E$ & $*$ & & * & * & $x$ & $x$ & \\
\hline$\omega \sigma \exists-\alpha E \theta=x$ & & & & & & & \\
\hline QDO 0 no $=20-$ & & & & & & $x$ & $x$ \\
\hline 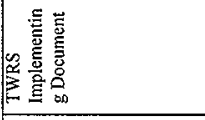 & 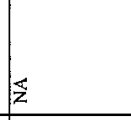 & & $\frac{\pi}{z}$ & $\frac{\pi}{z}$ & 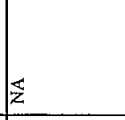 & $\Sigma$ & $\frac{\pi}{2}$ \\
\hline 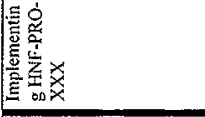 & $\underline{z}$ & & $\mathbb{z}$ & $\frac{\pi}{z}$ & $z$ & $\underline{z}$ & $\frac{\pi}{z}$ \\
\hline$z<$ & $x$ & & $x$ & $\times$ & $x$ & $x$ & $x$ \\
\hline 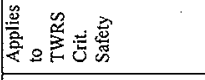 & & & & & & & \\
\hline 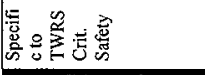 & & & & & & & \\
\hline 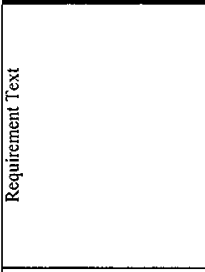 & 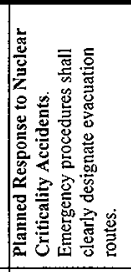 & 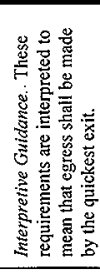 & 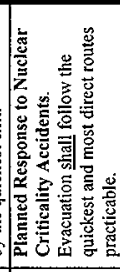 & 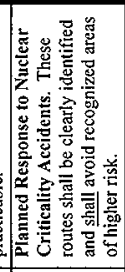 & 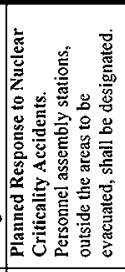 & 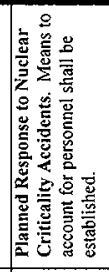 & 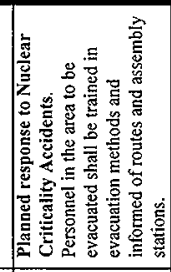 \\
\hline 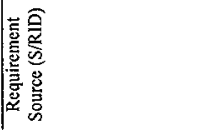 & & & & & & & \\
\hline 窝 & 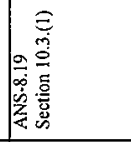 & & 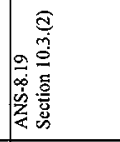 & 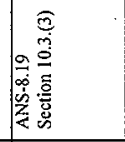 & 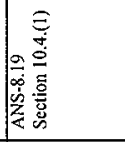 & 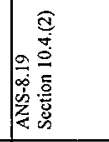 & 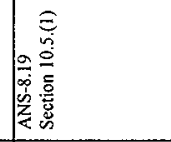 \\
\hline 言 & $\begin{array}{l}3 \\
0 \\
0 \\
0 \\
0 \\
0 \\
\end{array}$ & & 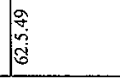 & 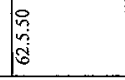 & 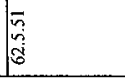 & $\mid \begin{array}{l}0 \\
0 \\
0 \\
0 \\
0\end{array}$ & :3 \\
\hline
\end{tabular}




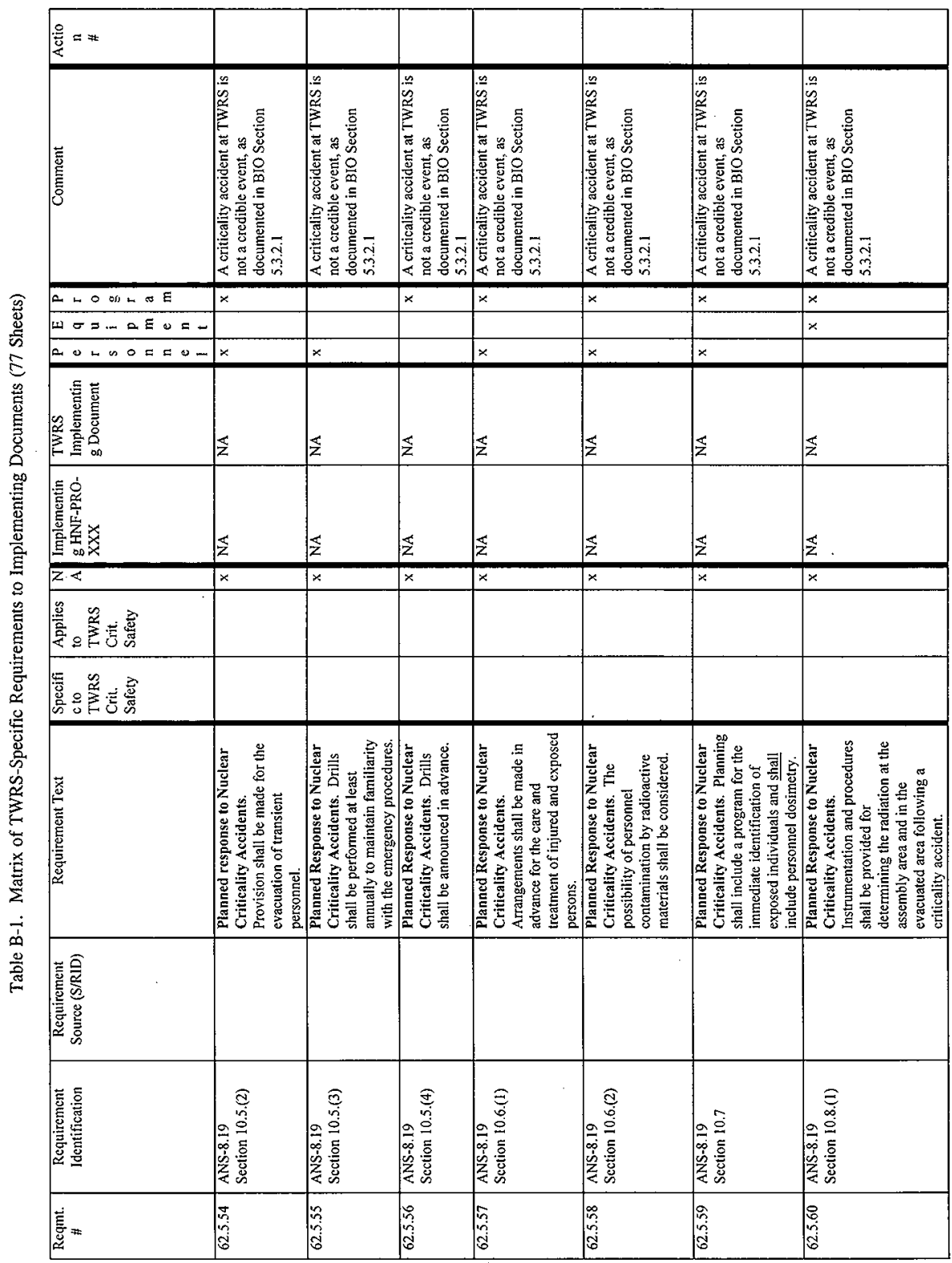


TWR-3721 Rev. 0

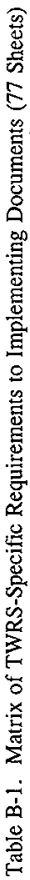

욜 =

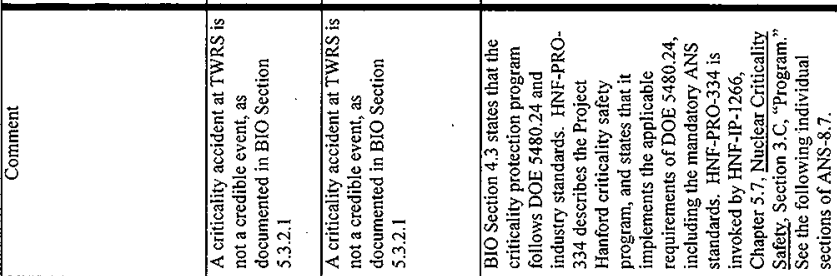

\begin{tabular}{|c|c|c|c|c|c|}
\hline $2 L O 0 D L \approx E$ & $x$ & $x$ & & 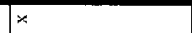 & $x$ \\
\hline$\omega \sigma=-\infty \quad \Xi 0=-$ & & & & & \\
\hline a o n en $0=0=0-$ & & & & & \\
\hline 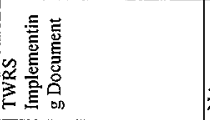 & z & $\mathbb{Z}$ & 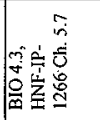 & 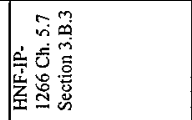 & 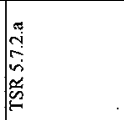 \\
\hline 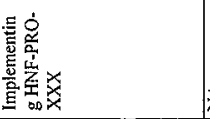 & $\ll$ & 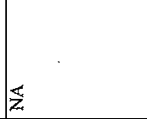 & 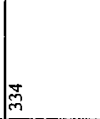 & 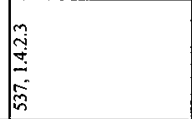 & 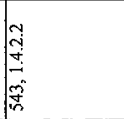 \\
\hline$z<$ & $x$ & $x$ & & & \\
\hline 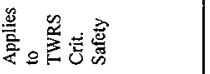 & & & & & \\
\hline 密密意 & & & $x$ & $x$ & $x$ \\
\hline 范 & 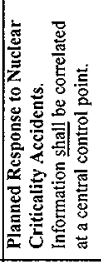 & 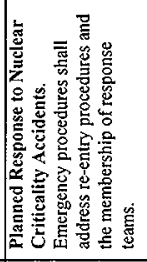 & 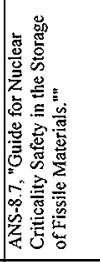 & 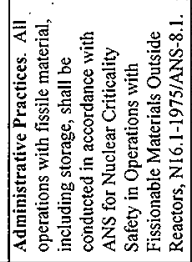 & 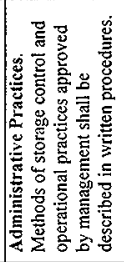 \\
\hline 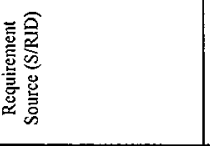 & & . & 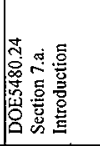 & & \\
\hline 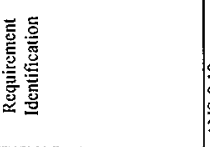 & 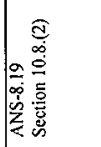 & 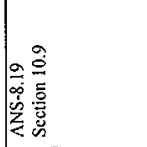 & 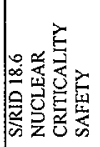 & 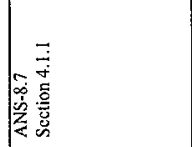 & 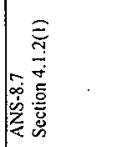 \\
\hline 䇋 & $\mid \begin{array}{l}\overrightarrow{0} \\
w \\
i \\
0 \\
0\end{array}$ & 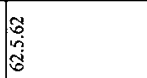 & 잉 & $\mid \begin{array}{l}5 \\
0 \\
0 \\
0 \\
0\end{array}$ & $\begin{array}{l}1 \\
0 \\
0 \\
0 \\
0\end{array}$ \\
\hline
\end{tabular}


TWR-3721 Rev, 0

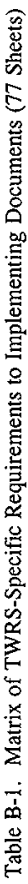

을 $\mathrm{E}=$

䓂

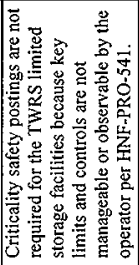

A 2 ODL $\operatorname{EE}$

एव $2-\alpha E \theta$

$\omega \omega-\infty \theta=0 \omega-x$

\begin{tabular}{|c|c|c|c|c|c|c|}
\hline 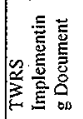 & 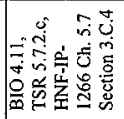 & 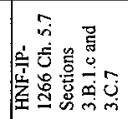 & 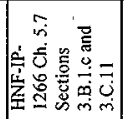 & 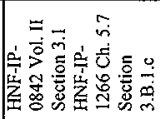 & 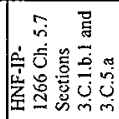 & 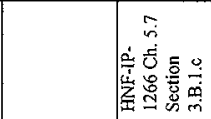 \\
\hline 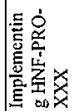 & 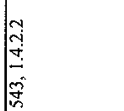 & 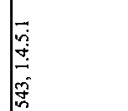 & 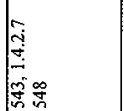 & 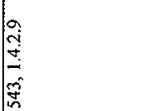 & 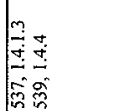 & $\frac{m}{m}$ \\
\hline
\end{tabular}

\begin{tabular}{|c|c|c|c|c|c|c|c|}
\hline$z<$ & & & & & & & \\
\hline 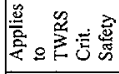 & $\star$ & & & $\times$ & & & \\
\hline 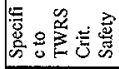 & & $\times$ & $x$ & & $x$ & $x$ & $x$ \\
\hline 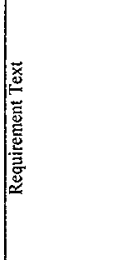 & 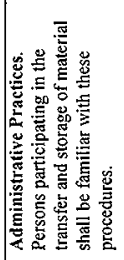 & 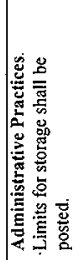 & 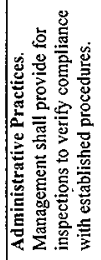 & 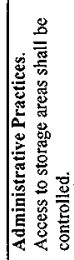 & 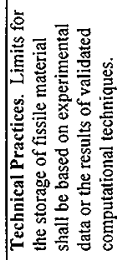 & 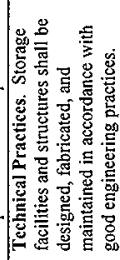 & 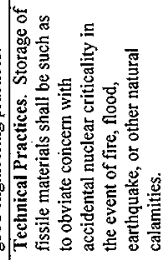 \\
\hline 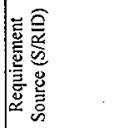 & & & & & & & . \\
\hline 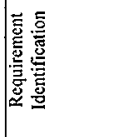 & 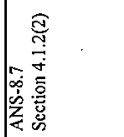 & 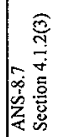 & 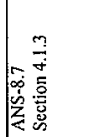 & 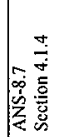 & 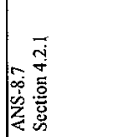 & 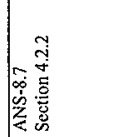 & 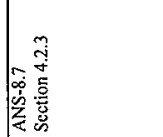 \\
\hline 套 & $\begin{array}{l}3 \\
0 \\
0 \\
0 \\
0 \\
0\end{array}$ & $\begin{array}{l}0 \\
0 \\
0 \\
0 \\
0 \\
0\end{array}$ & 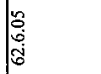 & $\mid$ & 占 & $\begin{array}{l}0 \\
8 \\
0 \\
0 \\
0 \\
0\end{array}$ & $\begin{array}{l}8 \\
0 \\
0 \\
0 \\
0\end{array}$ \\
\hline
\end{tabular}

Page B-61 
TWR-3721 Rev. 0

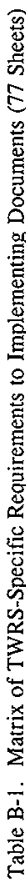

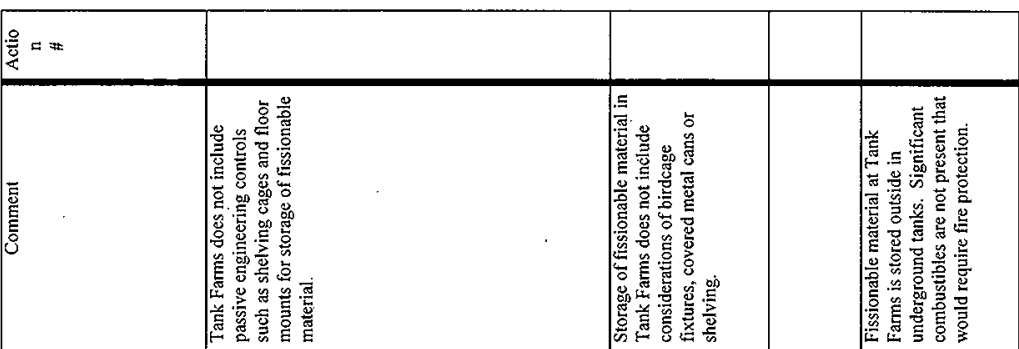

용

\begin{tabular}{|c|c|c|c|c|}
\hline H & & & & . \\
\hline 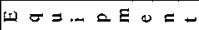 & $x$ & $x$ & $x$ & $x$ \\
\hline $20 \leq n 0=00$ & & & & \\
\hline 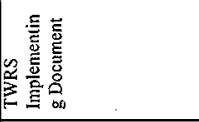 & $\Sigma$ & $\bar{z}$ & 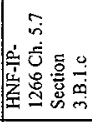 & $\frac{1}{z}$ \\
\hline 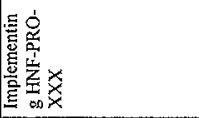 & 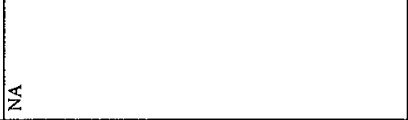 & $\bar{z}$ & 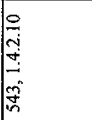 & $z$ \\
\hline z & $x$ & $x$ & & $x$ \\
\hline 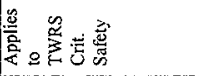 & & & & \\
\hline 总。芯芯总 & . & & $x$ & \\
\hline 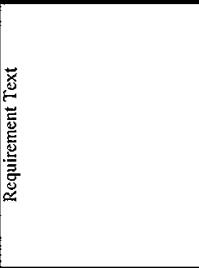 & 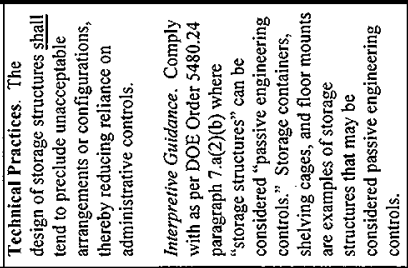 & 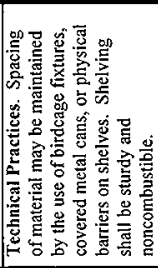 & 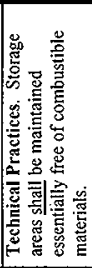 & 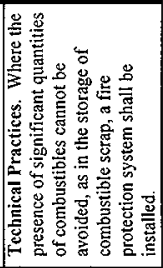 \\
\hline 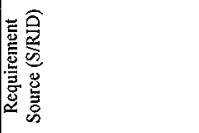 & , & & & \\
\hline 駕总 & 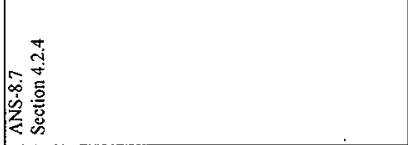 & 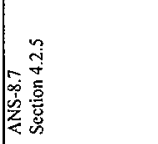 & 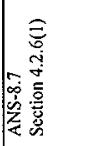 & 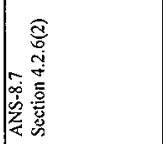 \\
\hline$\underset{\mathscr{E}}{\stackrel{\Xi}{E}}$ & $\begin{array}{l}0 \\
0 \\
0 \\
0 \\
0\end{array}$ & $\begin{array}{l}= \\
\overline{0} \\
\text { ci } \\
0\end{array}$ & $\begin{array}{l}\approx \\
0 \\
0 \\
0 \\
0\end{array}$ & ๙2 \\
\hline
\end{tabular}




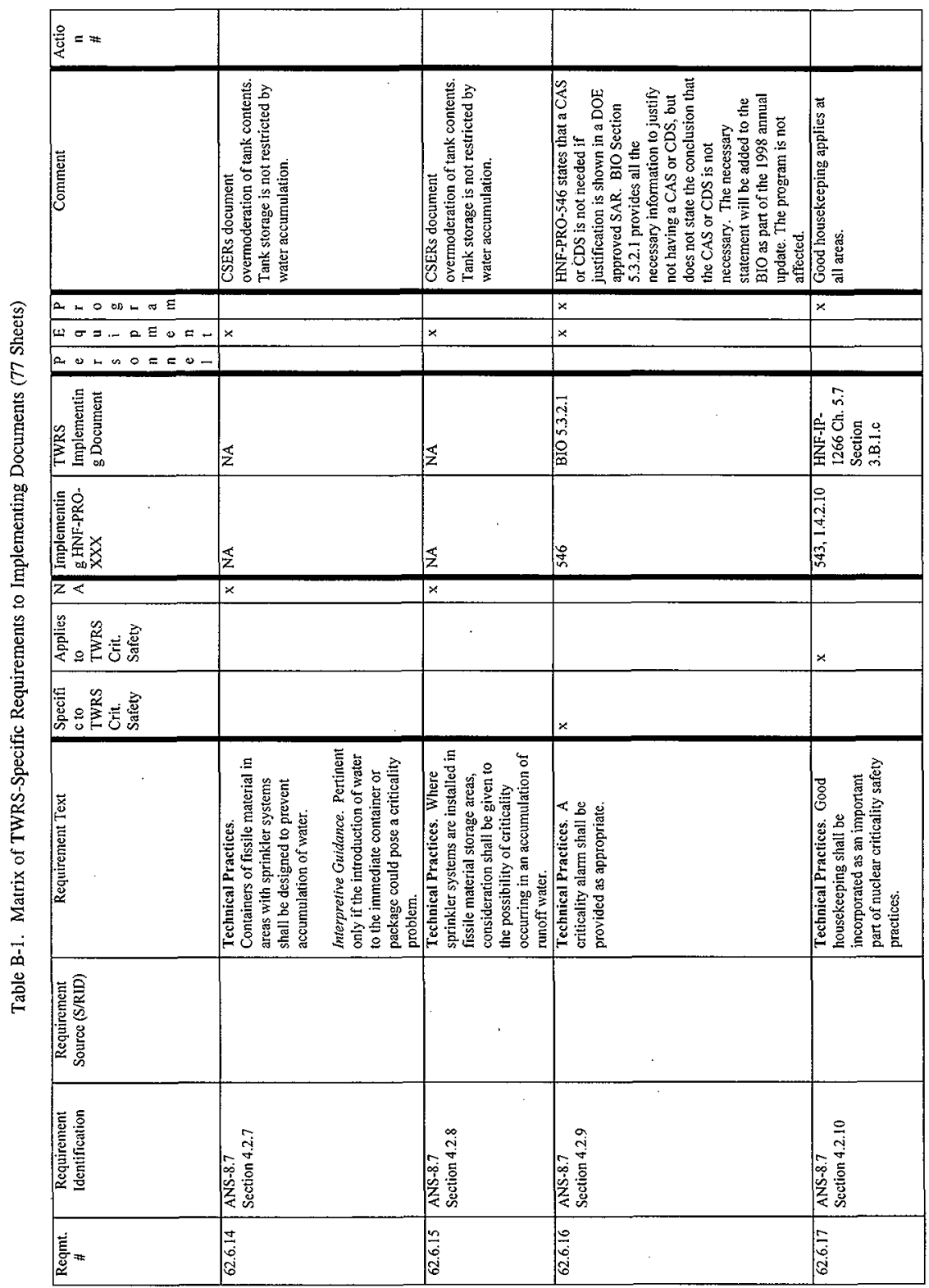


TWR-3721 Rev. 0

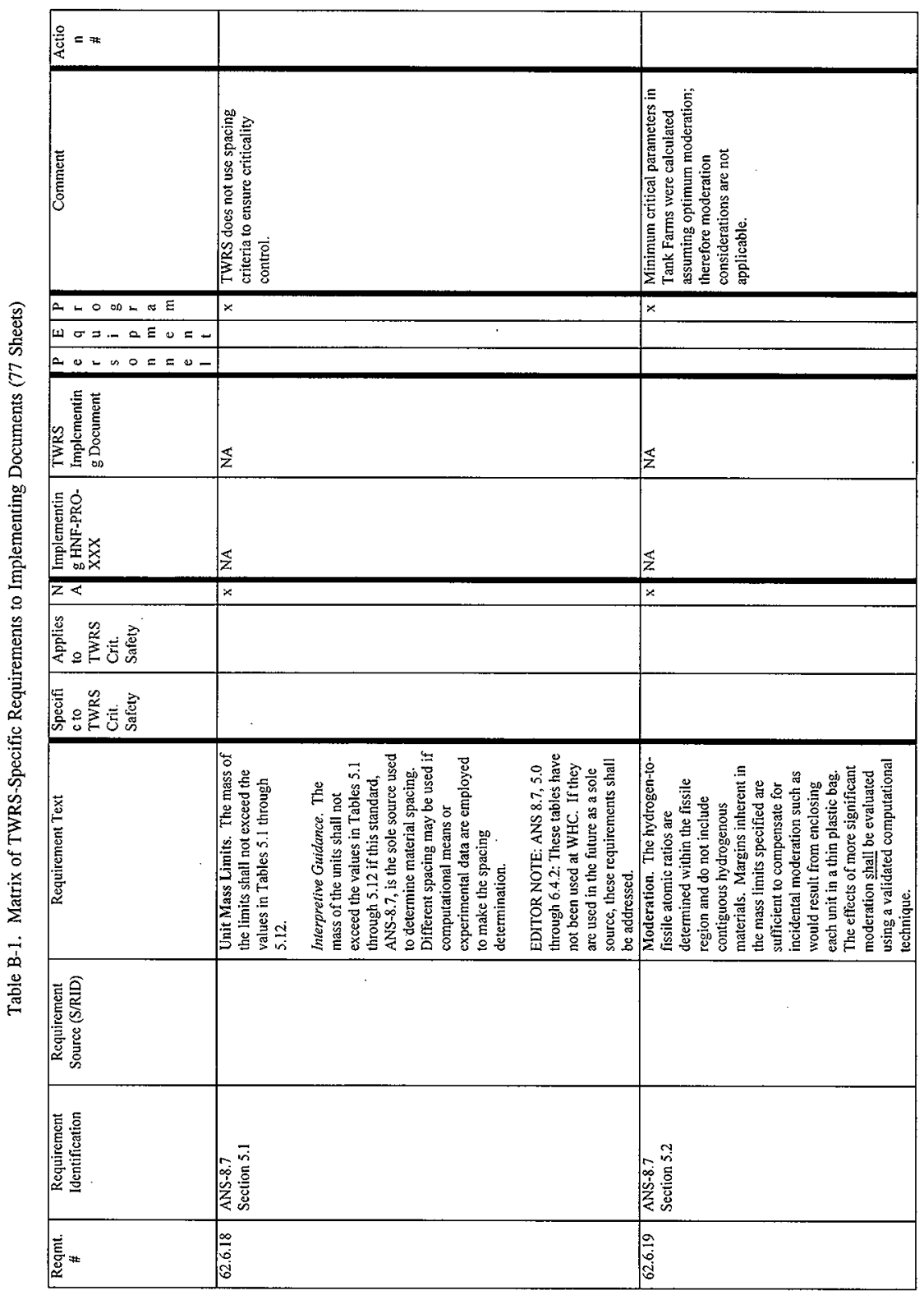


TWR-3721 Rev. 0

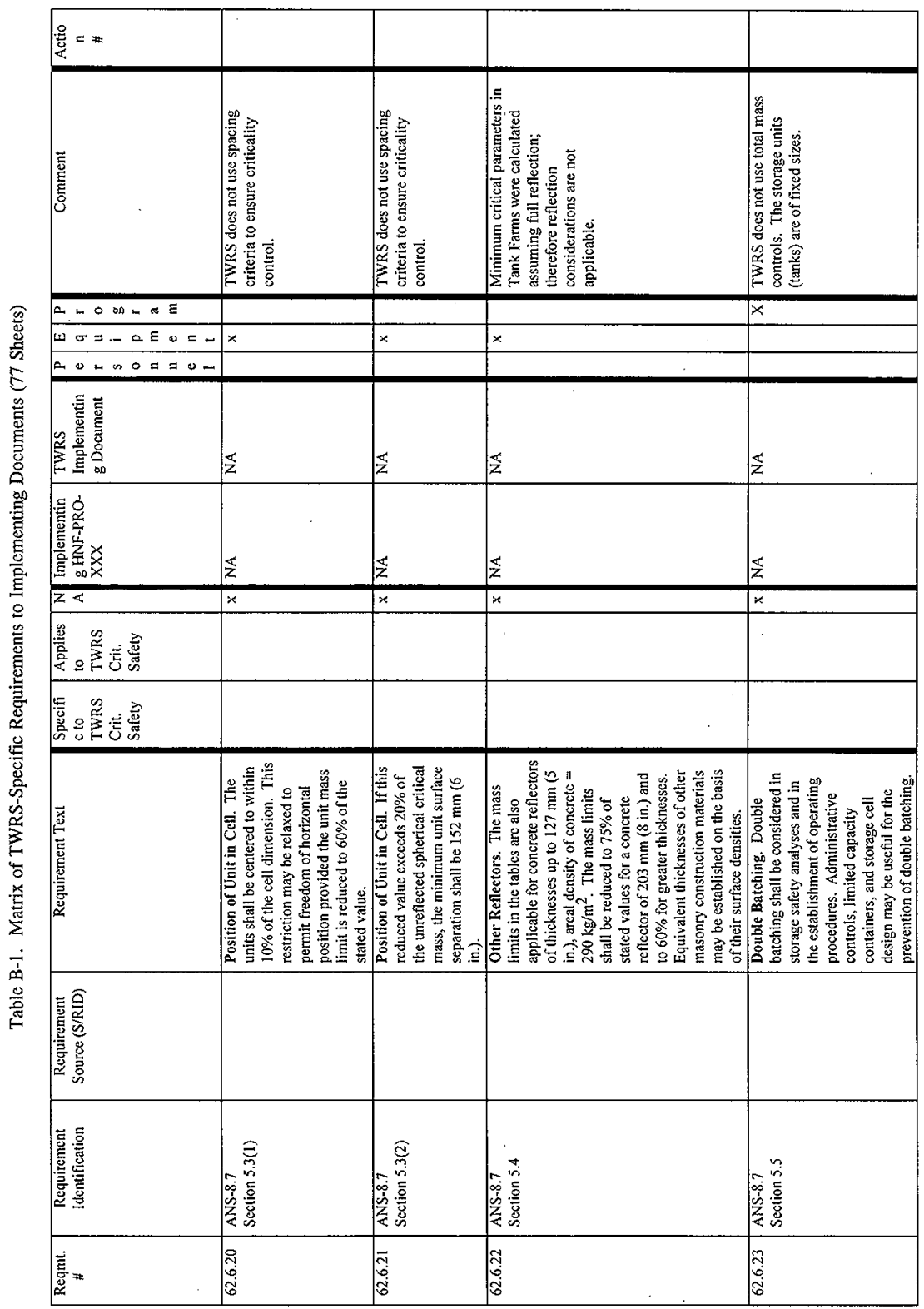

Page B-65 


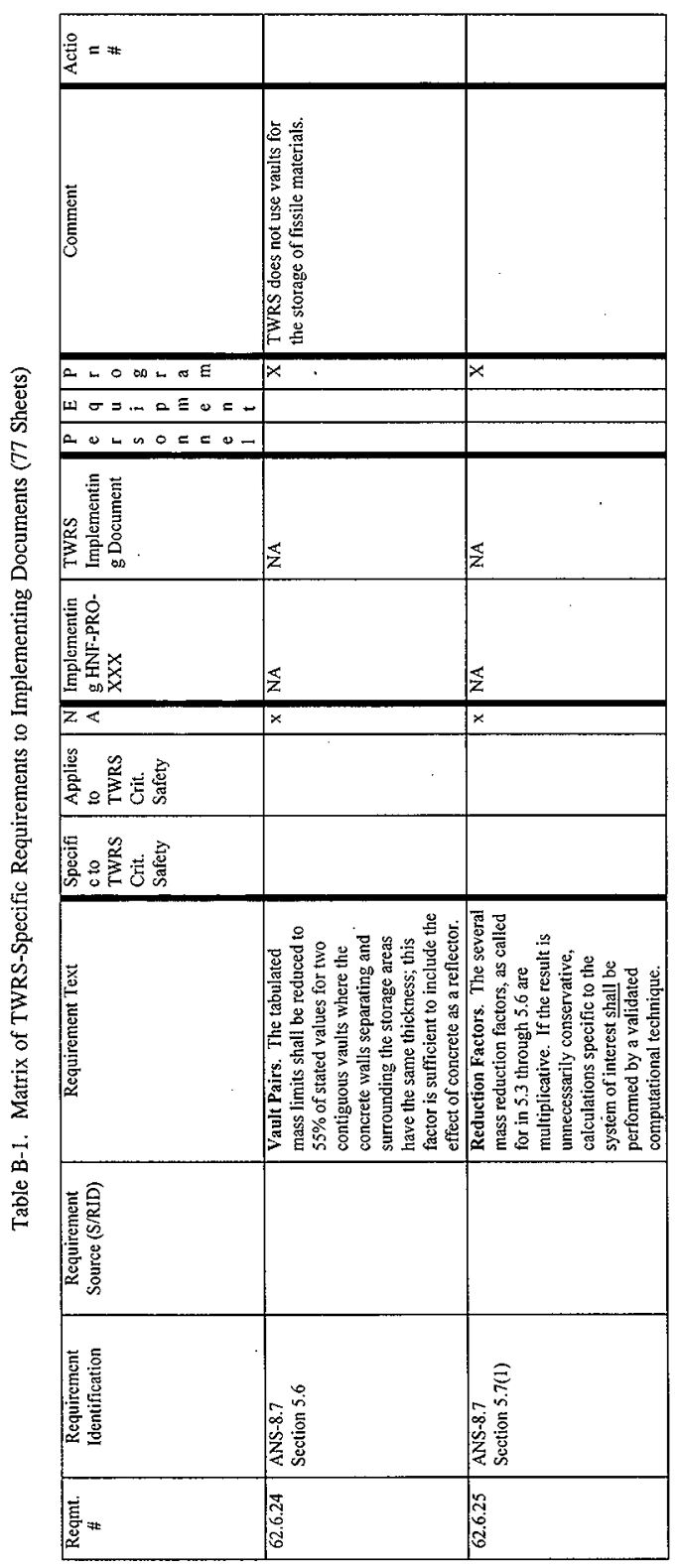

Page B-66 


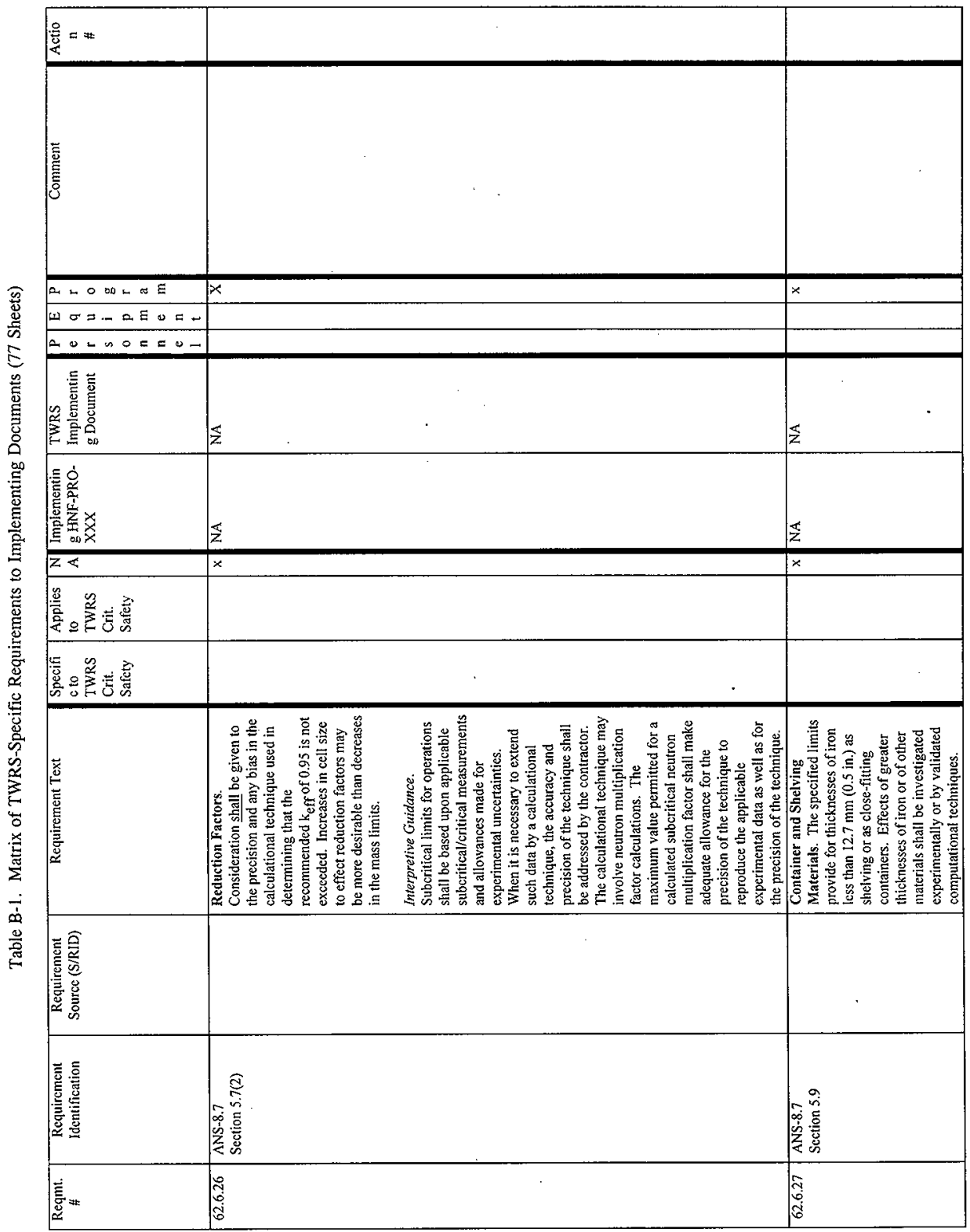


TWR-3721 Rev. 0

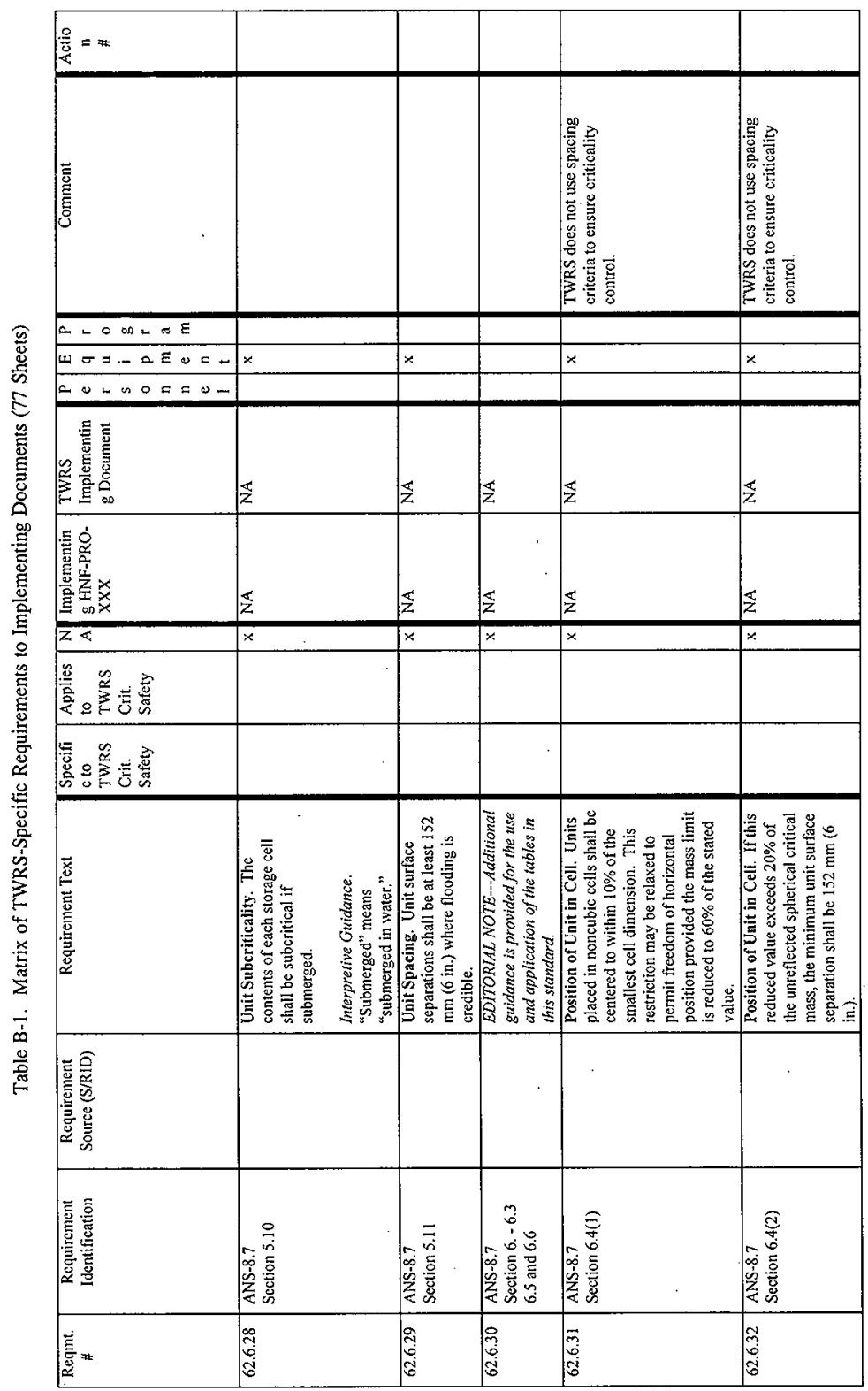


TWR-3721 Rev. 0

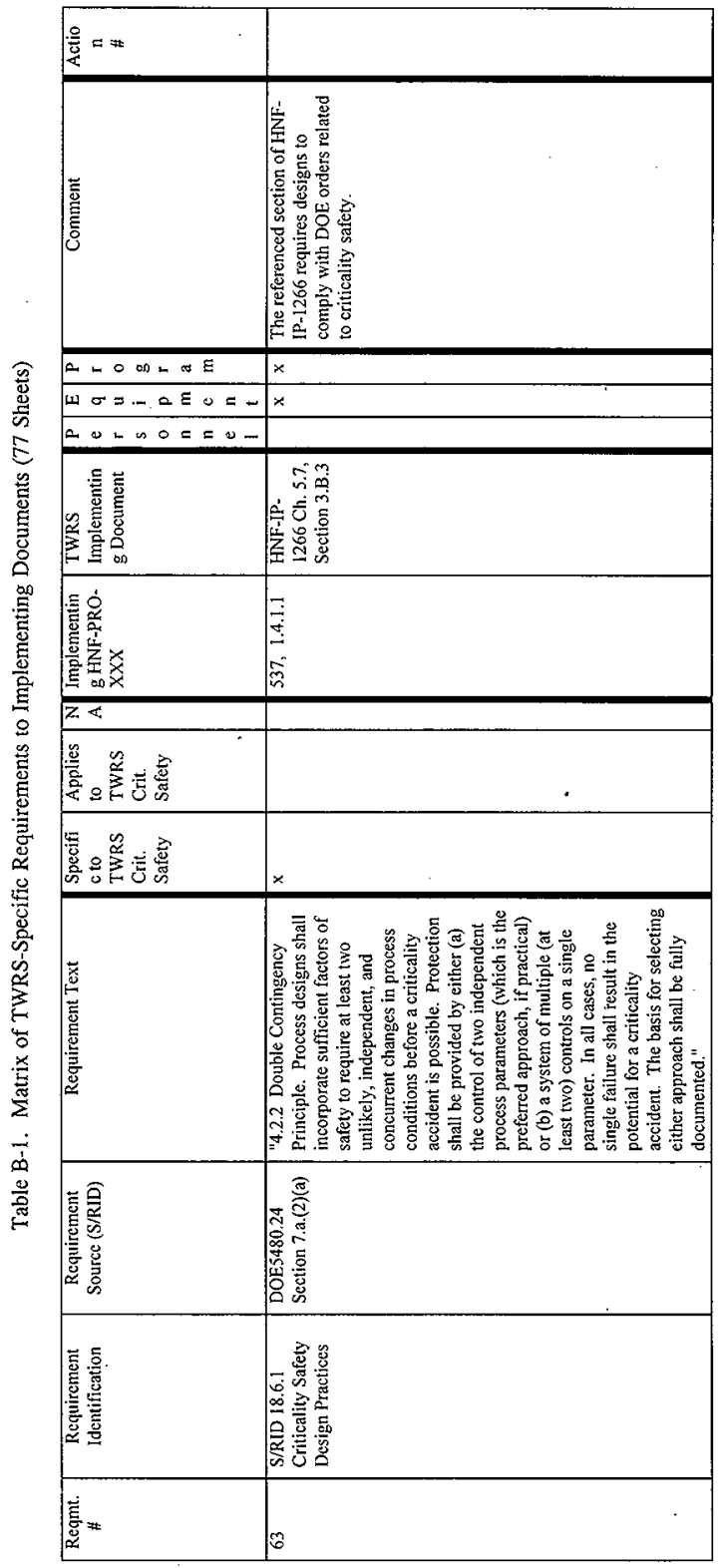

Page B-69 
TWR-3721 Rev. 0

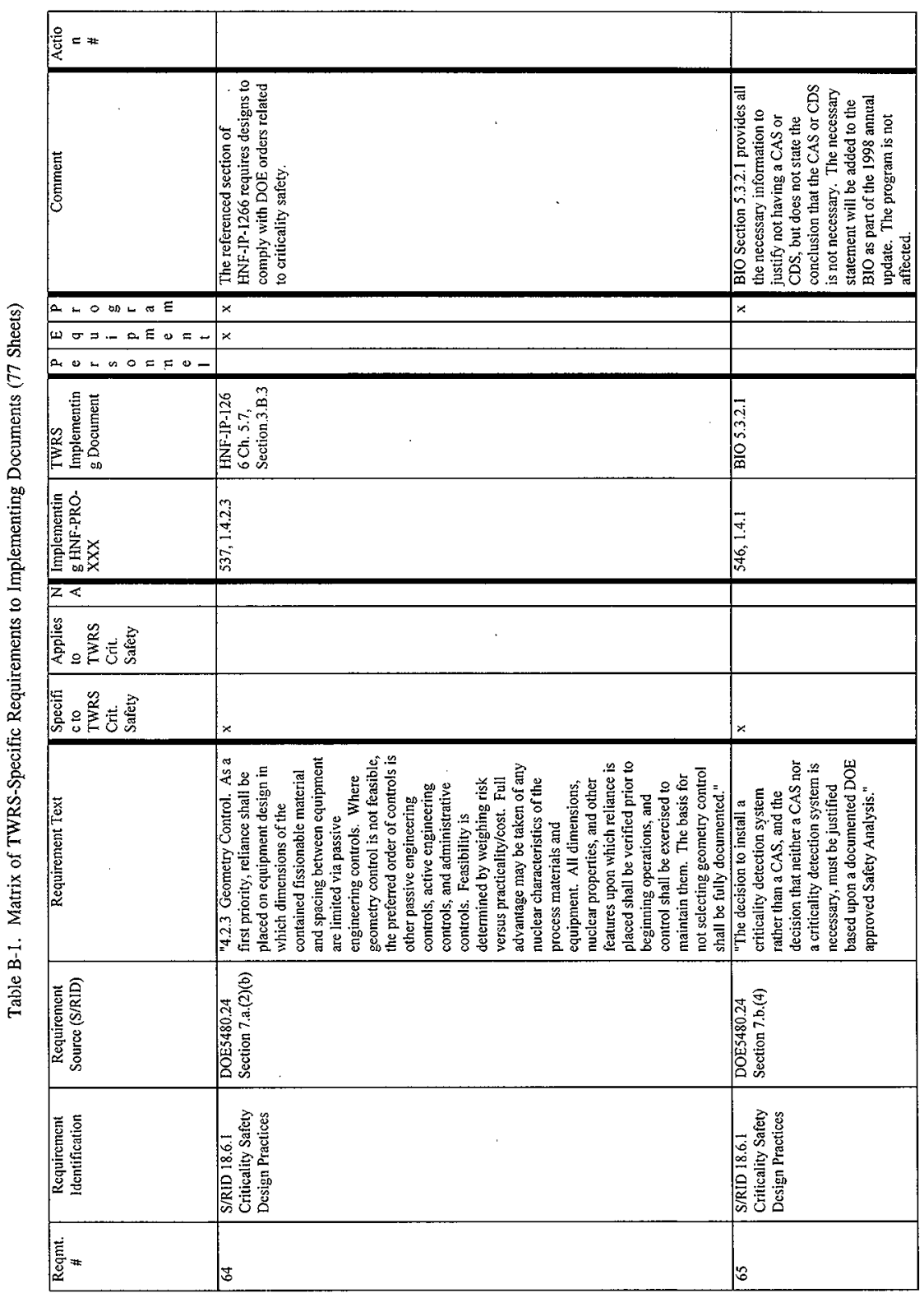


TWR-3721 Rev. 0

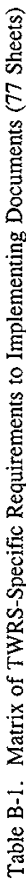
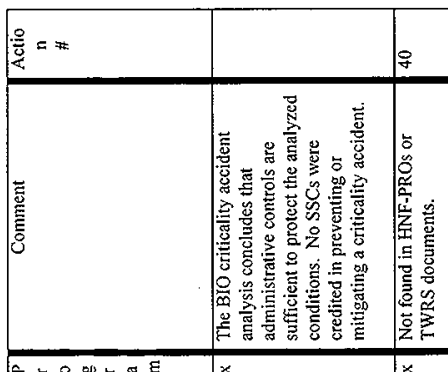

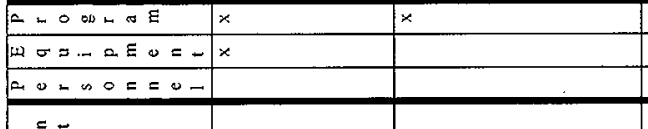

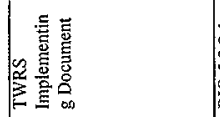

\section{言过}

वis

تิن

$x$

$\pi<$

을

究品

它蓄

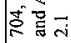

$\cong$

$z$

\begin{tabular}{|c|}
\hline 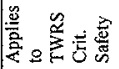 \\
\hline 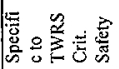 \\
\hline
\end{tabular}

$x$

\begin{tabular}{|c|c|c|c|c|c|}
\hline & 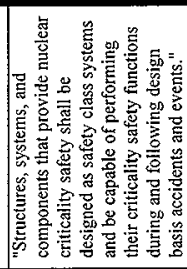 & 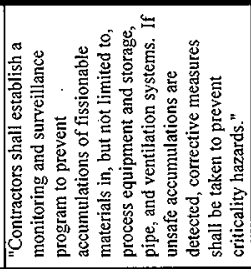 & 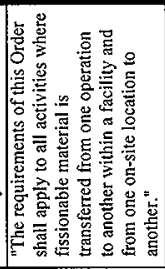 & 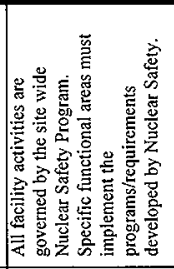 & 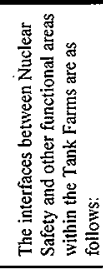 \\
\hline & 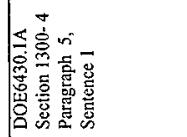 & 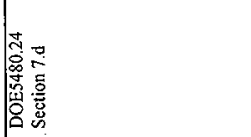 & 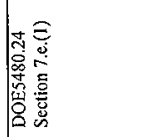 & 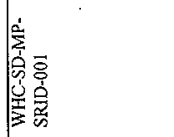 & \\
\hline & 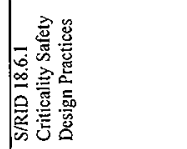 & 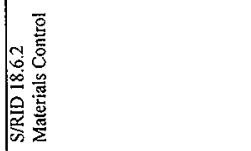 & 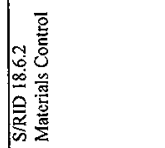 & 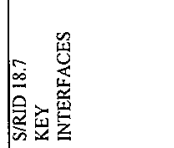 & \\
\hline & 8 & 5 & $\infty$ & 18 & \\
\hline
\end{tabular}




\begin{tabular}{|c|c|c|c|c|}
\hline 尊 = & & & & \\
\hline 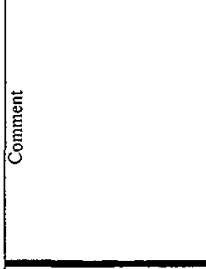 & & & & 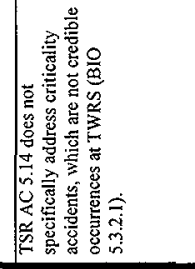 \\
\hline$A-O$ DD $\sim \pi$ & $x$ & $x$ & $x$ & $x$ \\
\hline$\Psi \sigma a \ldots a \leq 0=$ & & & & \\
\hline$a-\infty=0=E 0$ & & & & \\
\hline 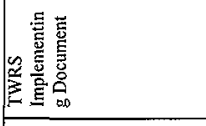 & 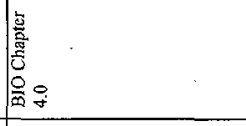 & 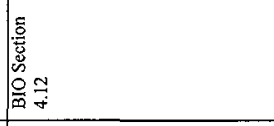 & 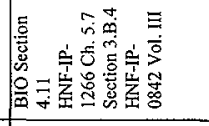 & 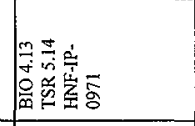 \\
\hline 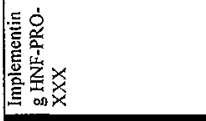 & & & & \\
\hline $2 \ll$ & & & & \\
\hline 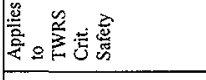 & $x$ & $x$ & $x$ & \\
\hline 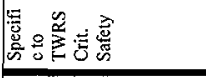 & & & & $x$ \\
\hline 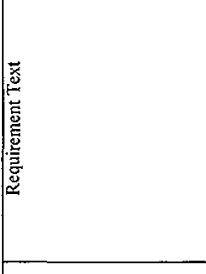 & 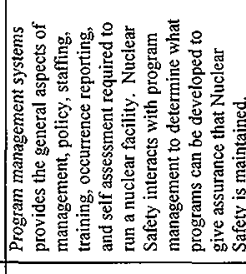 & 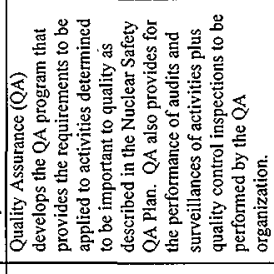 & 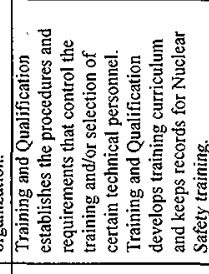 & 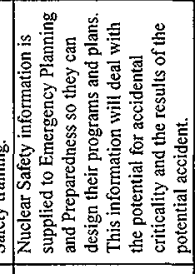 \\
\hline 㖣 & 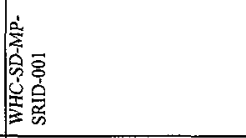 & 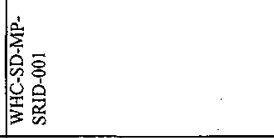 & 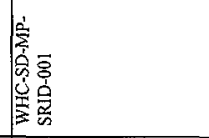 & 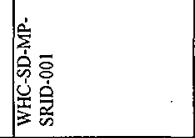 \\
\hline 焉 & 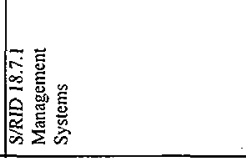 & 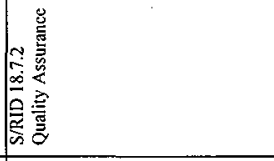 & 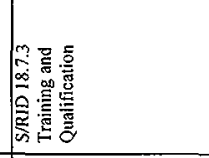 & 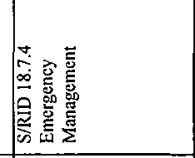 \\
\hline 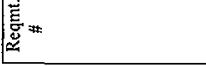 & iR & $E$ & $\approx$ & $\approx$ \\
\hline
\end{tabular}


TWR-3721 Rev. 0

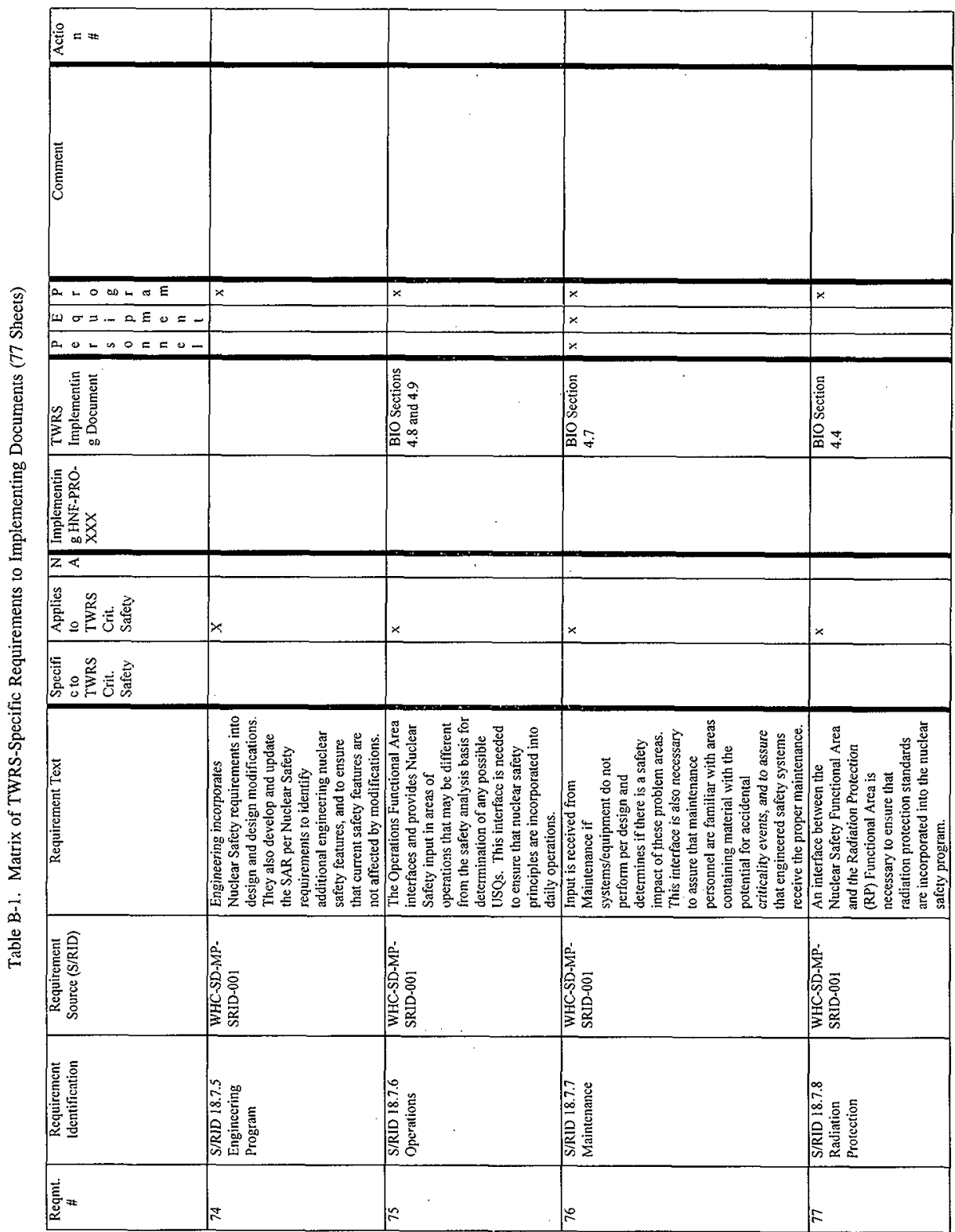




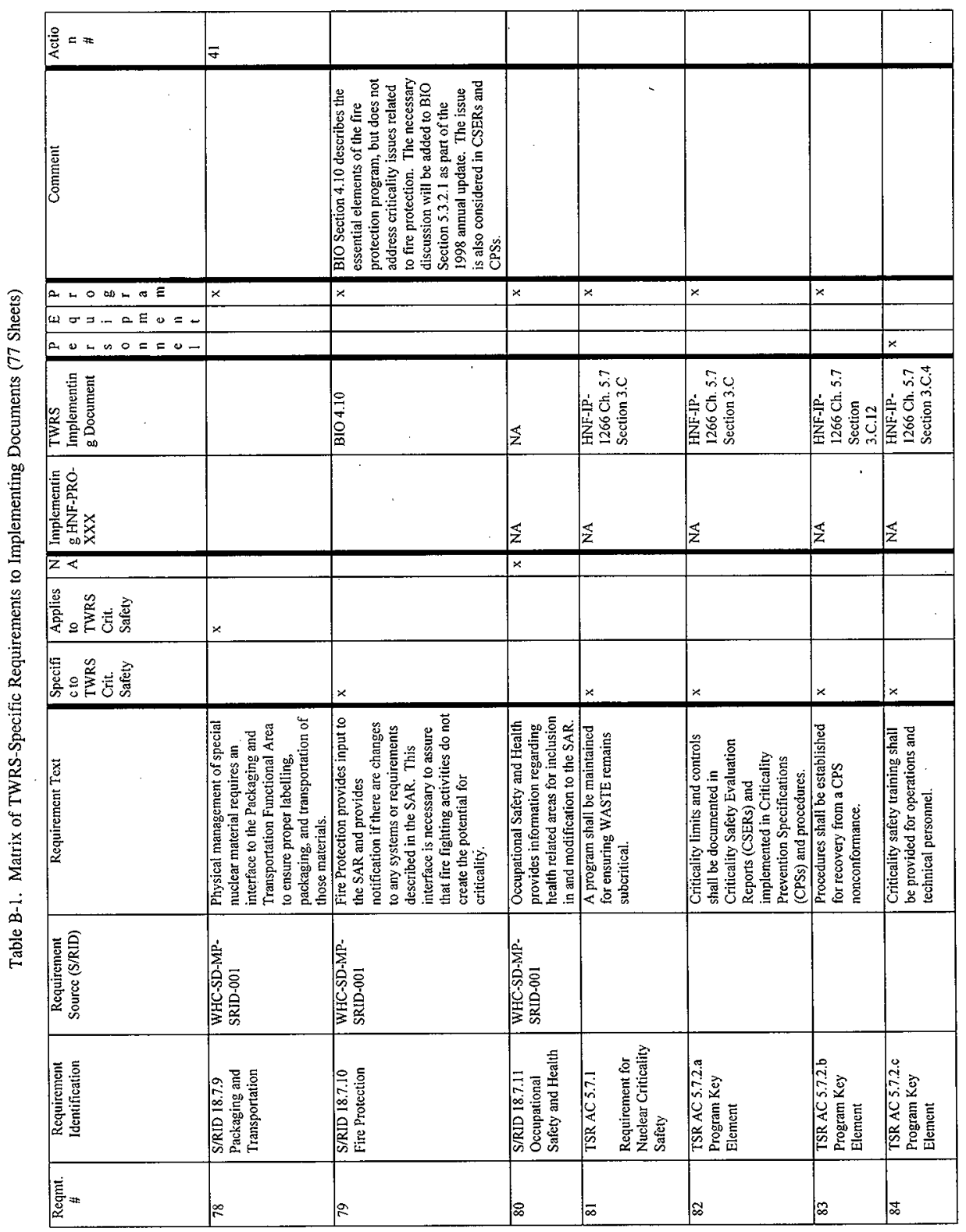


TWR-3721 Rev. 0

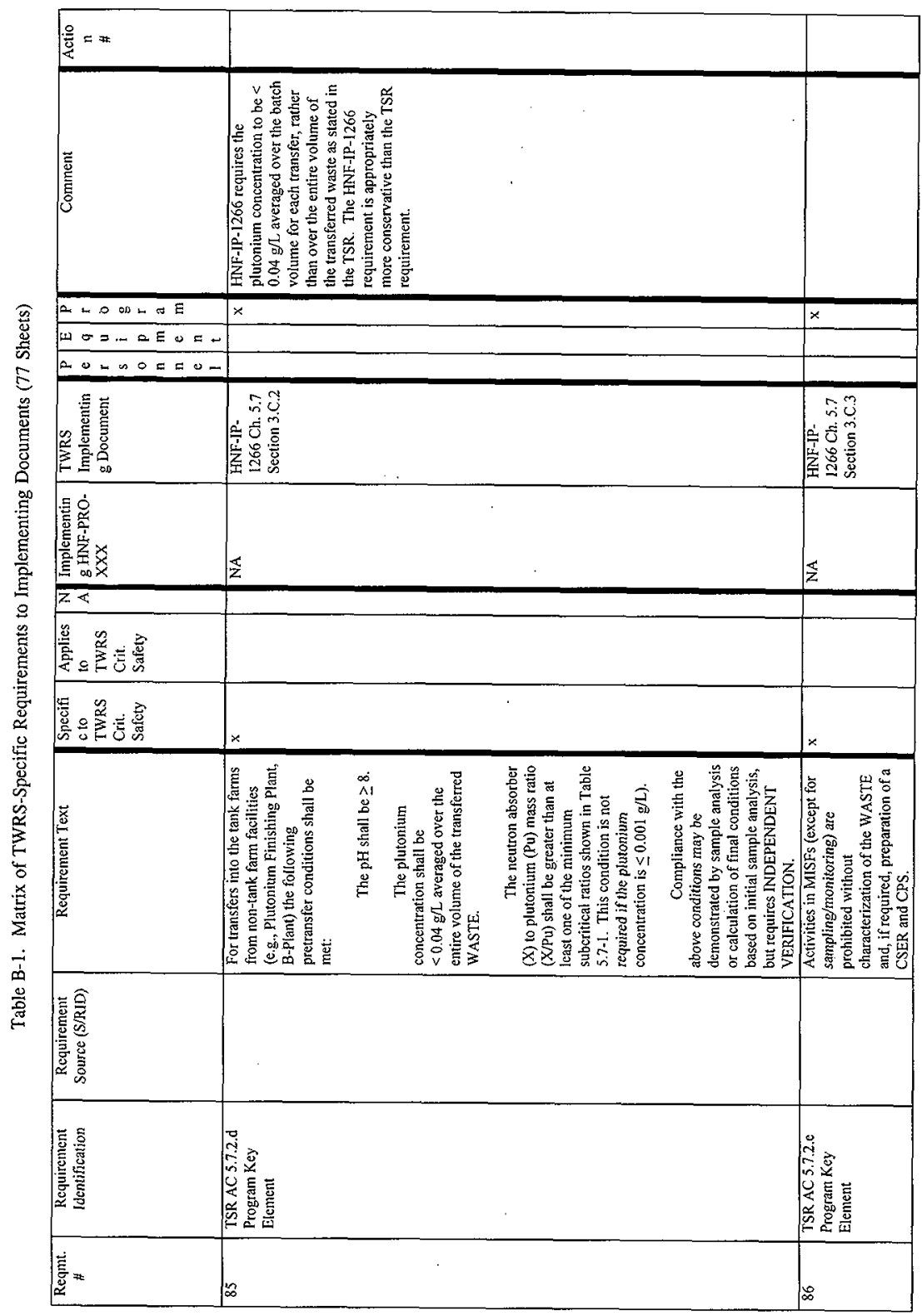

Page B-75 


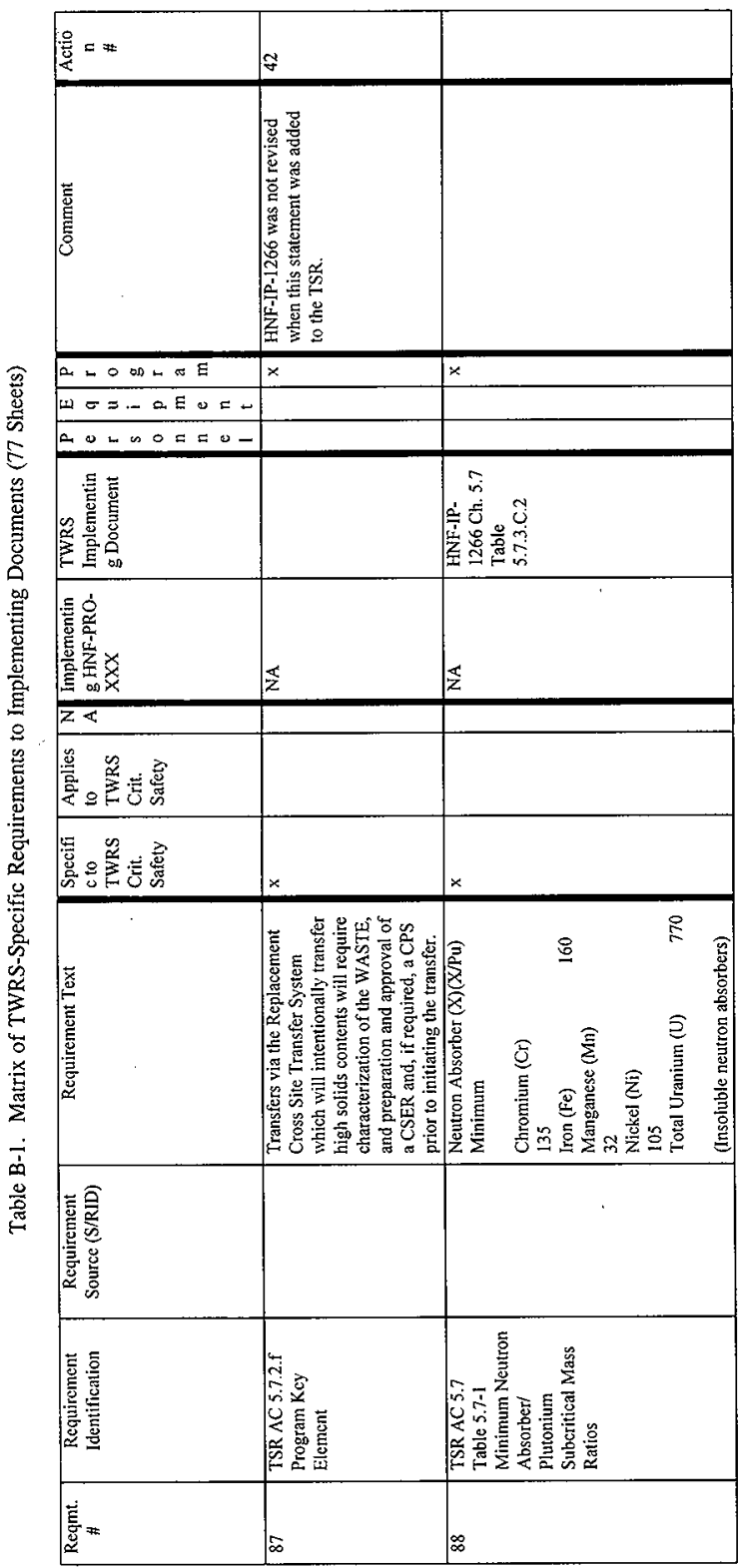

Page B-76 


\begin{tabular}{|c|c|c|c|}
\hline$=7$ & \% & & \\
\hline 言 & 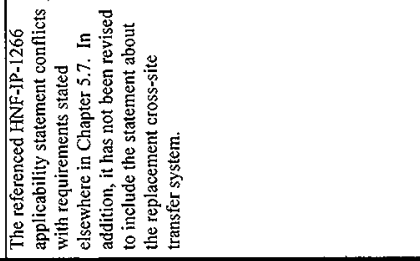 & . & \\
\hline$\alpha-0$ OD⿰幺幺 & $x$ & & \\
\hline$\omega \sigma=\ldots E 0=-$ & & & \\
\hline $00 \cos 0=00$ & & & \\
\hline 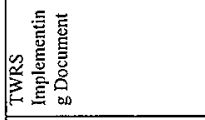 & 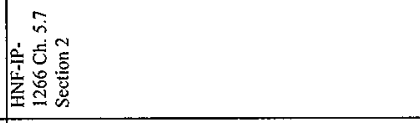 & & \\
\hline 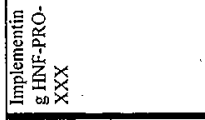 & $\$$ & & \\
\hline $2 \pi$ & & & \\
\hline 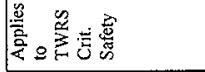 & & & \\
\hline 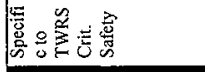 & $\approx$ & & \\
\hline 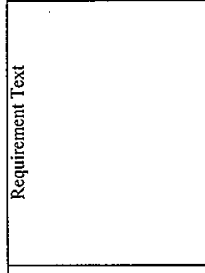 & 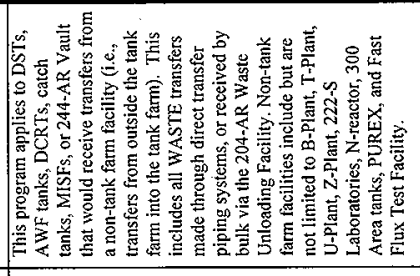 & 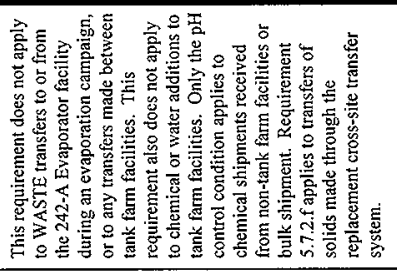 & 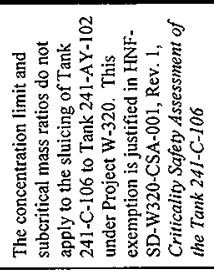 \\
\hline 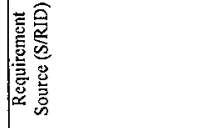 & & & \\
\hline 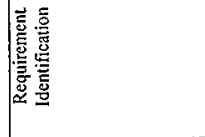 & 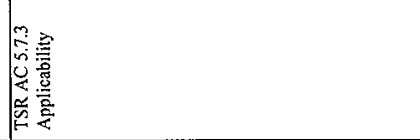 & & \\
\hline & $\infty$ & & \\
\hline
\end{tabular}


TWR-3721 Rev. 0

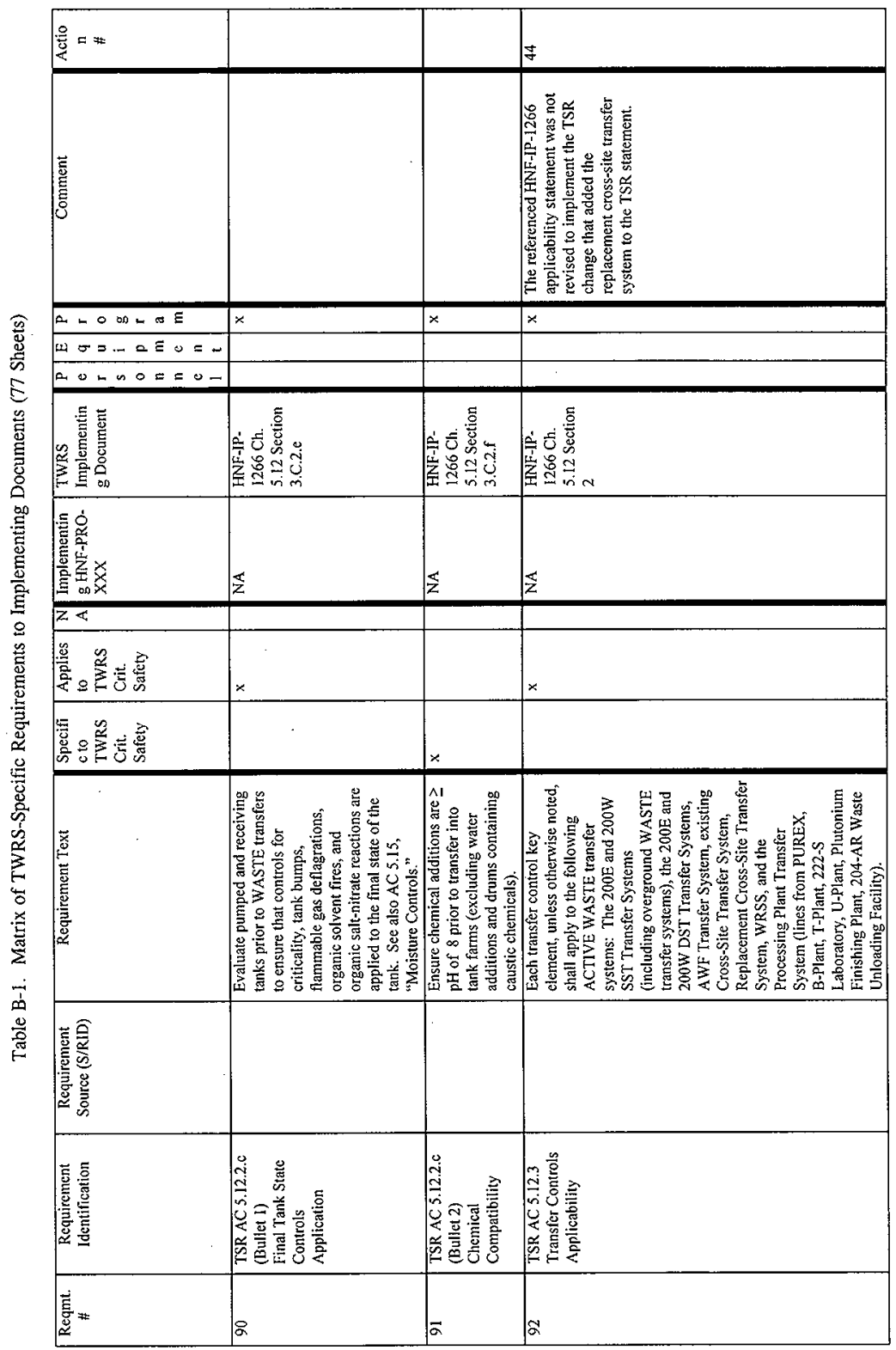


TWR-3721 Rev. 0

\section{APPENDIX C}

Matrix of Hanford Site (HNF-PRO) Requirements to TWRS Implementing Documents 
TWR-3721 Rev. 0

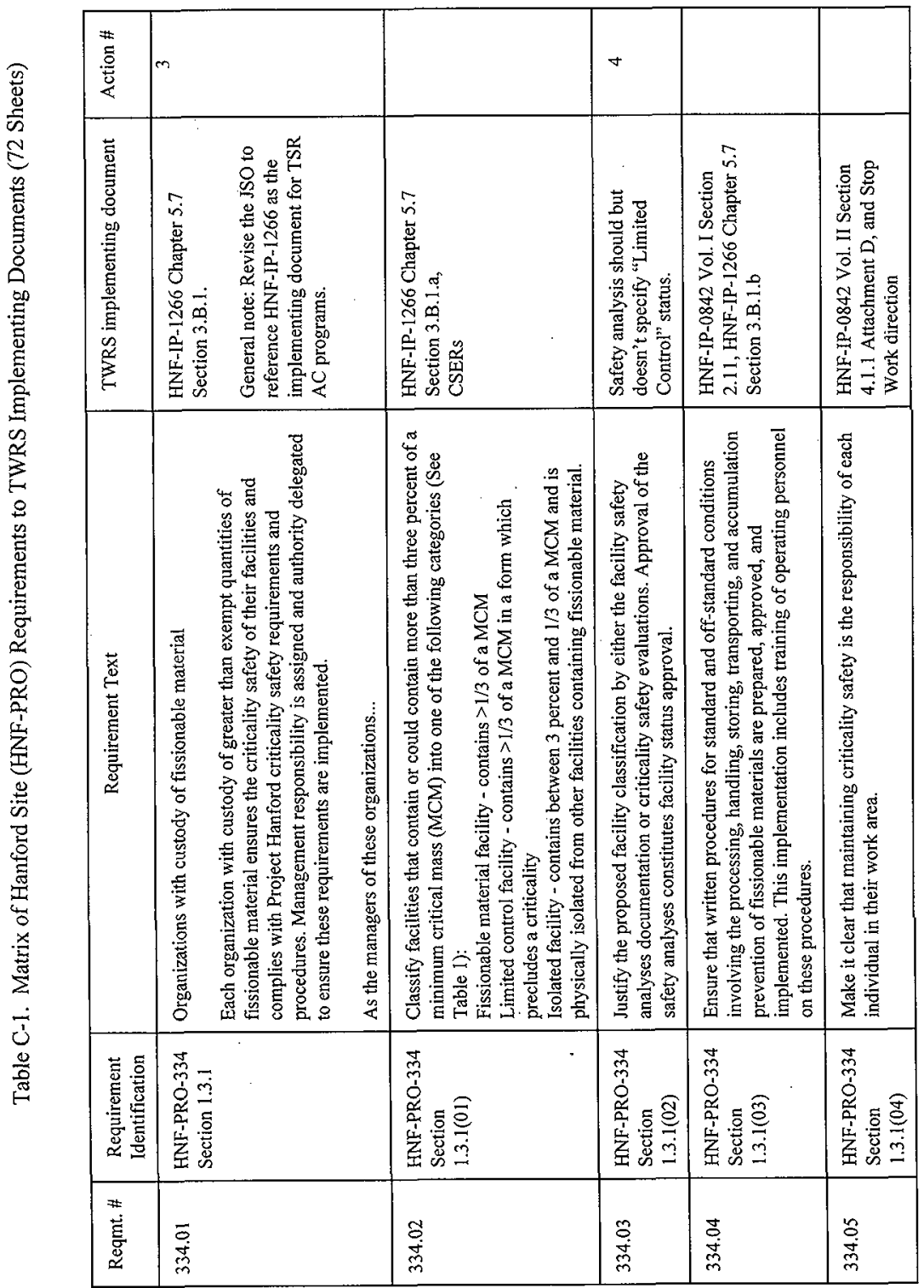


TWR-3721 Rev. 0

\begin{tabular}{|c|c|c|c|c|c|c|}
\hline 范 & 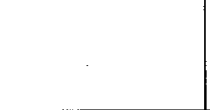 & & & & \multicolumn{2}{|l|}{ in } \\
\hline 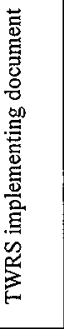 & 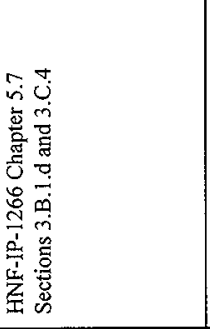 & 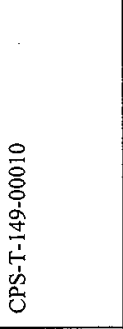 & 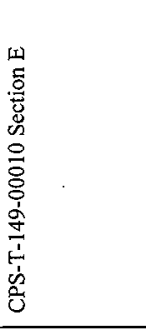 & 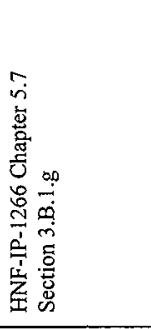 & 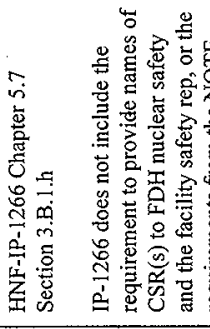 & \\
\hline 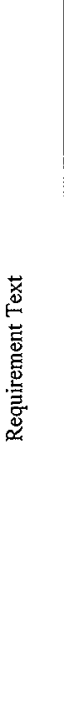 & 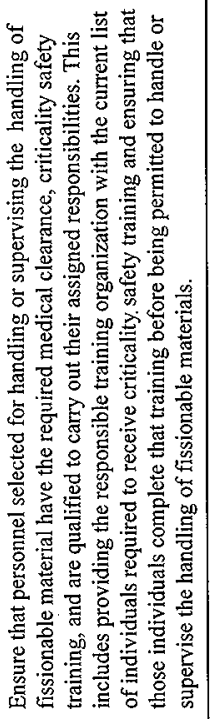 & 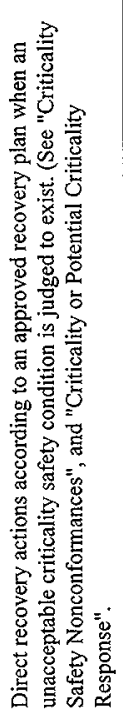 & 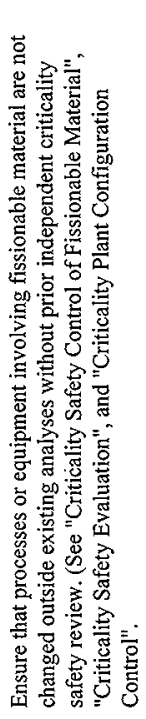 & 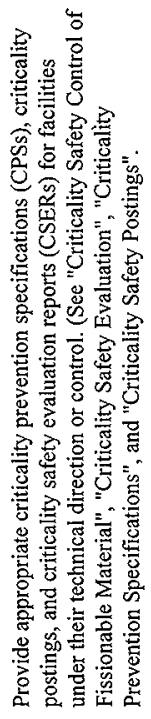 & 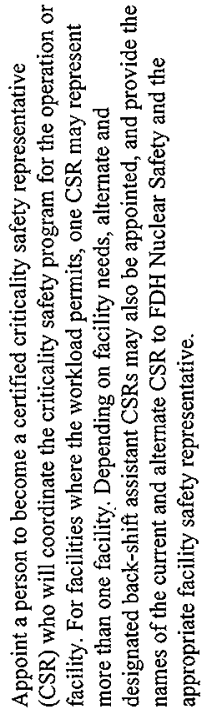 & 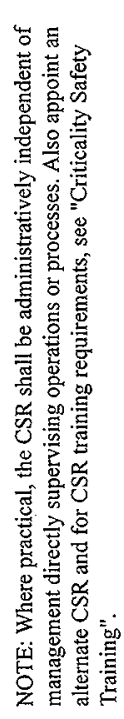 \\
\hline 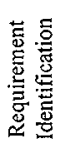 & 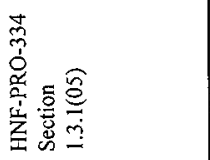 & 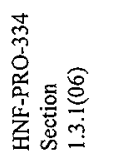 & 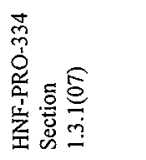 & 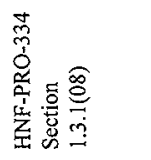 & 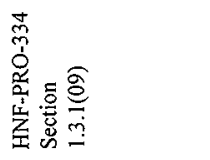 & \\
\hline 苞 & 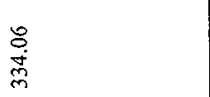 & $\begin{array}{l}5 \\
\text { iे } \\
\text { లે }\end{array}$ & $\begin{array}{l}\stackrel{\infty}{\circ} \\
\stackrel{\text { mे }}{m}\end{array}$ & $\begin{array}{l}\stackrel{g}{0} \\
\stackrel{j}{m} \\
m\end{array}$ & $\begin{array}{l}9 \\
\stackrel{\dot{q}}{m} \\
m\end{array}$ & \\
\hline
\end{tabular}


TWR-3721 Rev. 0

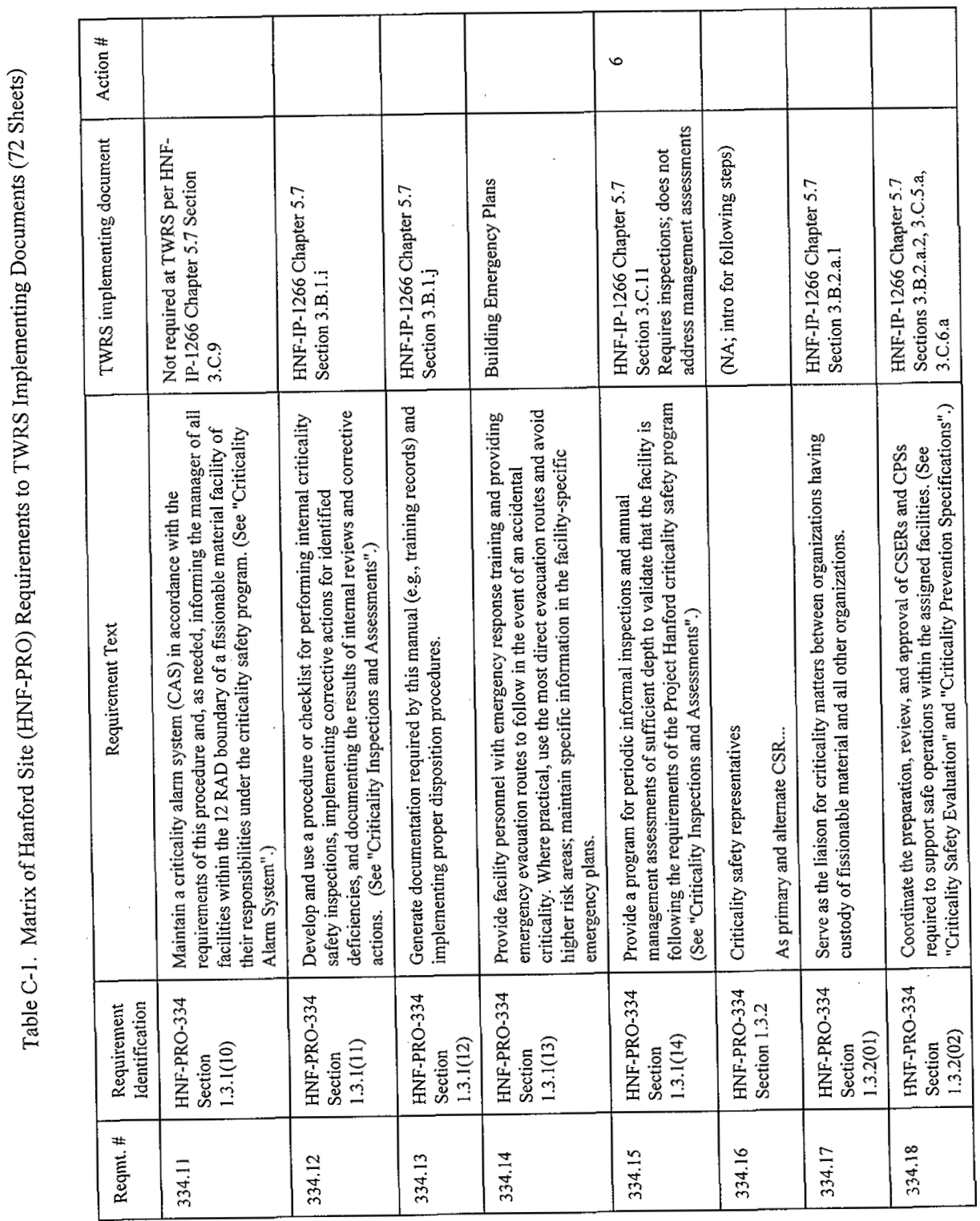

Page C-4 
TWR-3721 Rev. 0

\begin{tabular}{|c|c|c|c|c|c|c|c|c|c|}
\hline 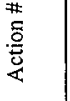 & & $r$ & & & & & & & \\
\hline 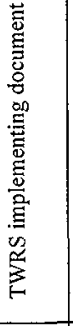 & 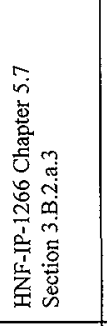 & 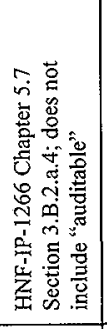 & 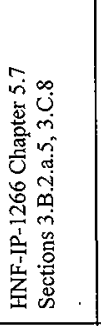 & 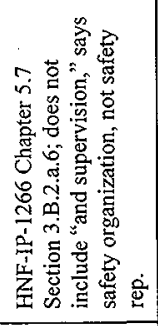 & 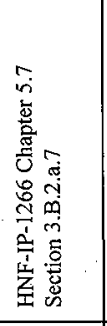 & 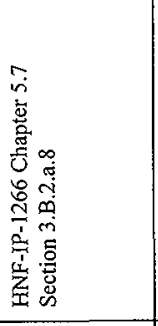 & 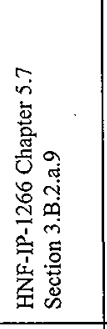 & 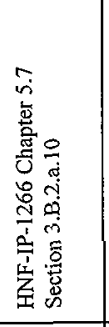 & 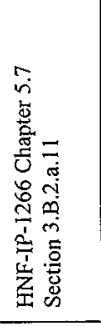 \\
\hline 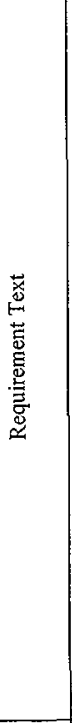 & 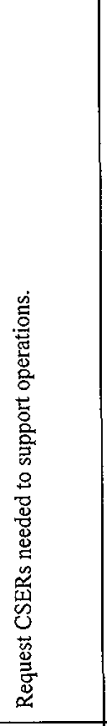 & 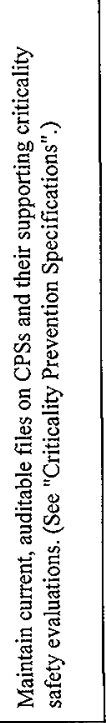 & 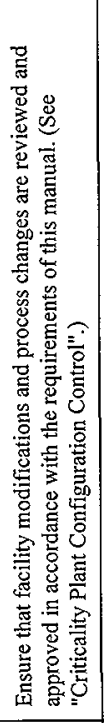 & 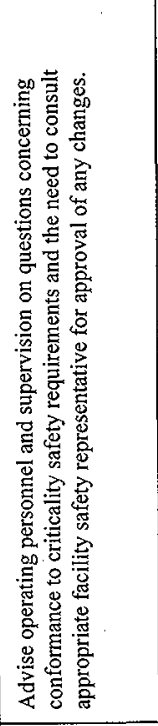 & 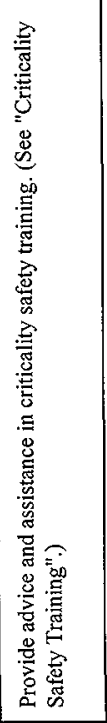 & 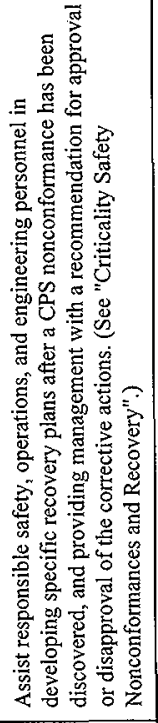 & 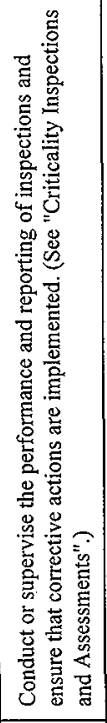 & 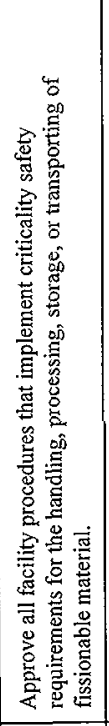 & 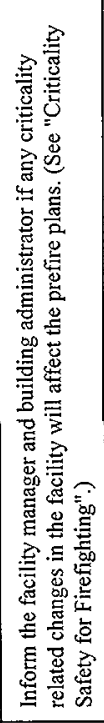 \\
\hline 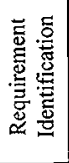 & 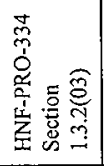 & 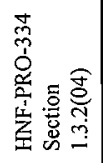 & 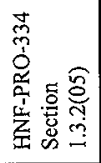 & 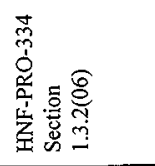 & 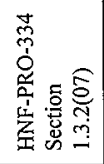 & 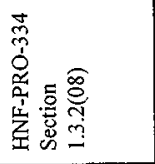 & 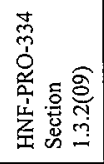 & 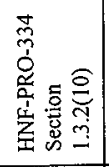 & 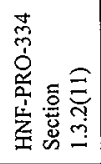 \\
\hline 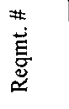 & $\frac{a}{\dot{m}}$ & ঙ্ণ & 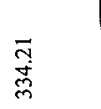 & సָ & 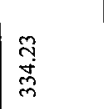 & $\begin{array}{l}\underset{j}{J} \\
\text { J }\end{array}$ & $\begin{array}{l}\stackrel{\sim}{y} \\
\vec{m}\end{array}$ & $\begin{array}{l}\stackrel{\text { I }}{\mathrm{j}} \\
\text { m }\end{array}$ & $\begin{array}{l}\tilde{y} \\
\text { d } \\
\text { d }\end{array}$ \\
\hline
\end{tabular}




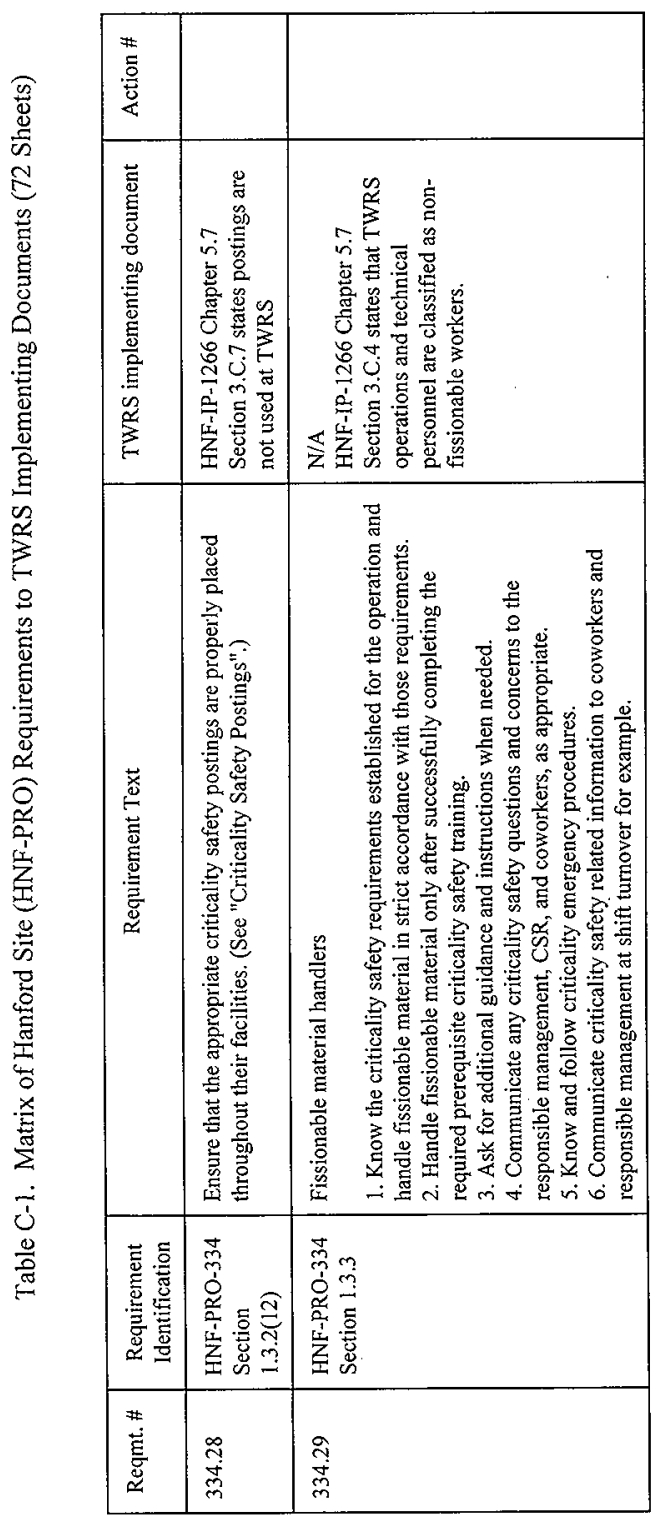

Page C-6 
TWR-3721 Rev. 0

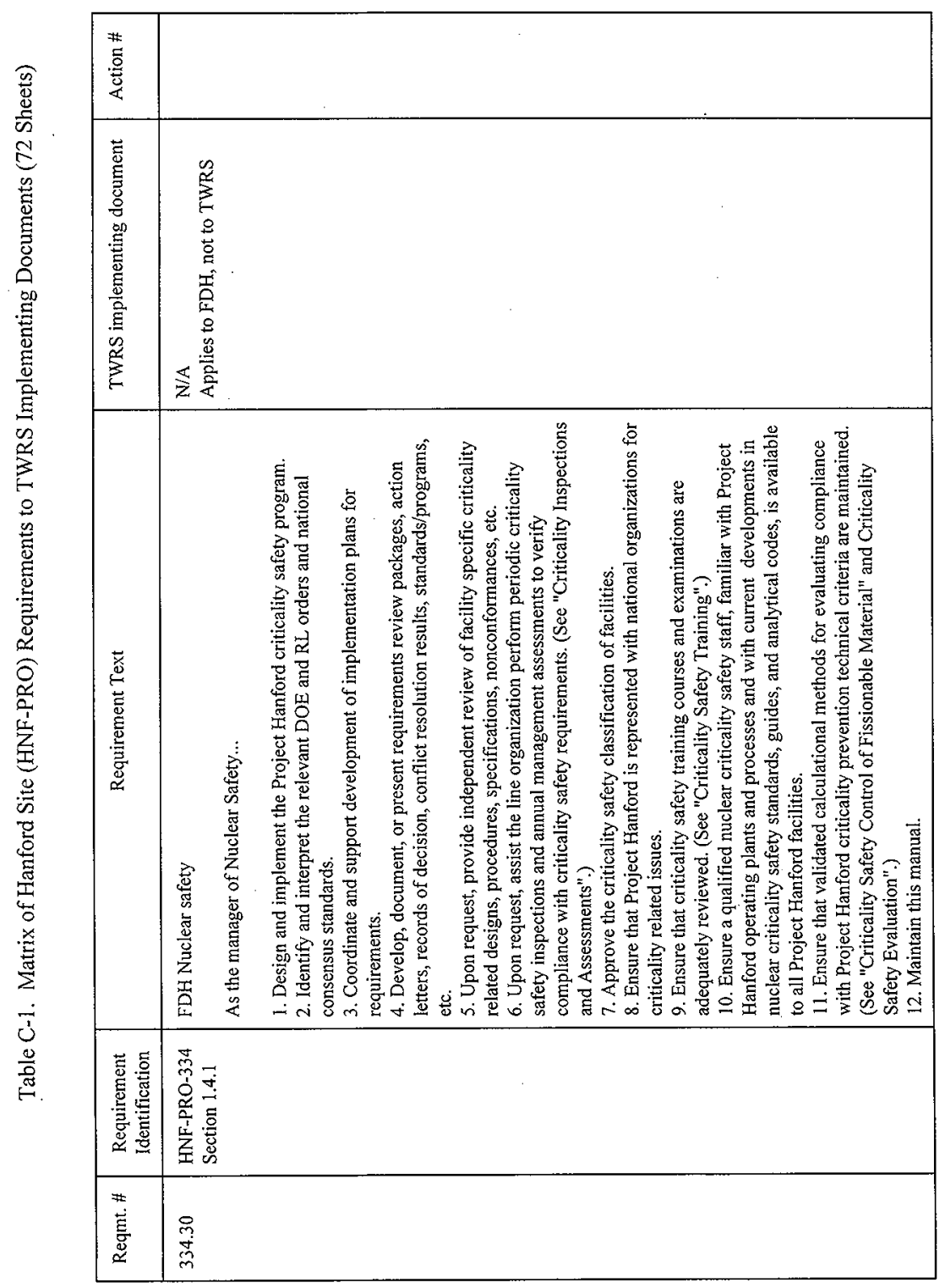

Page C-7 
TWR-3721 Rev. 0

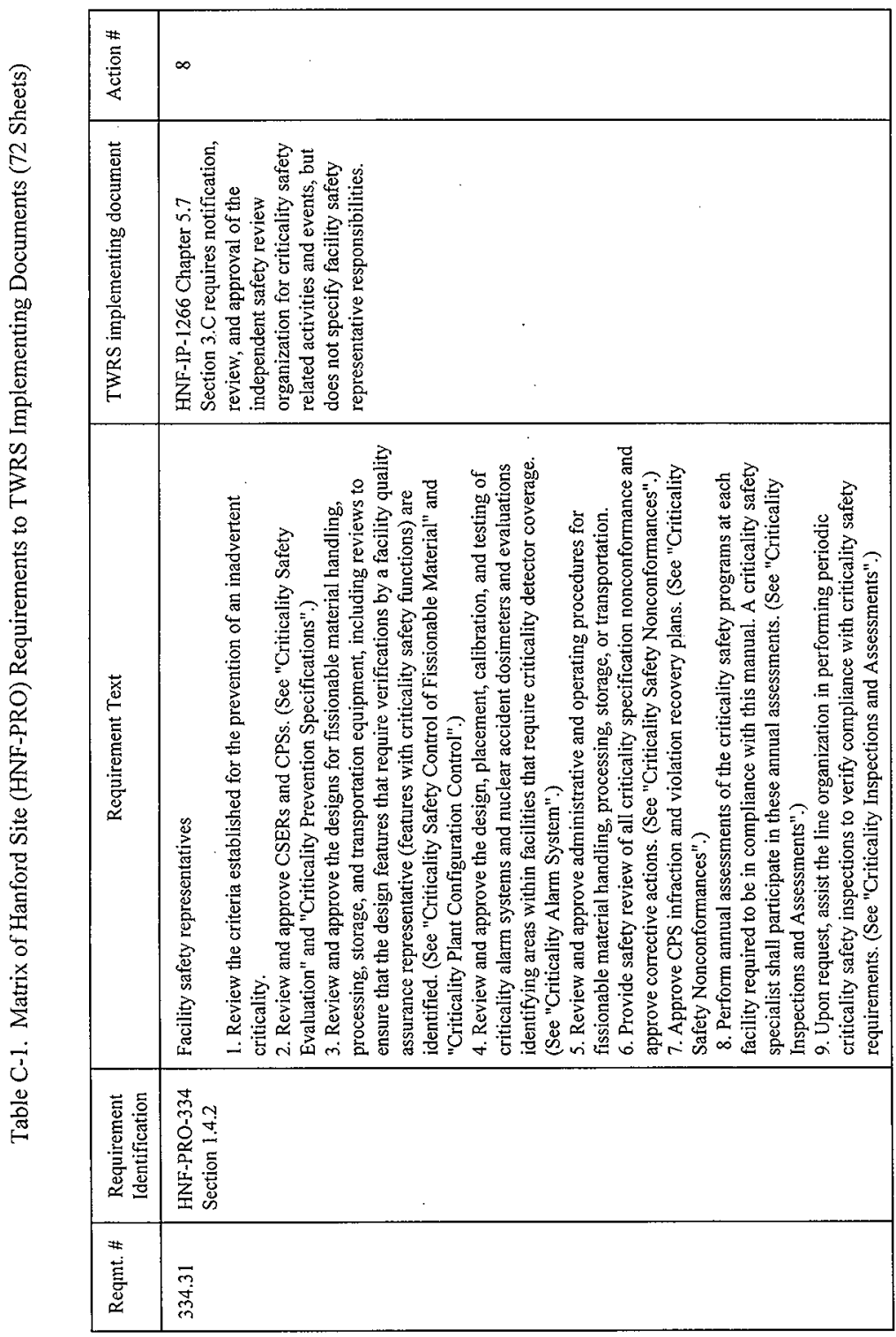

Page C-8 
TWR-3721 Rev. 0

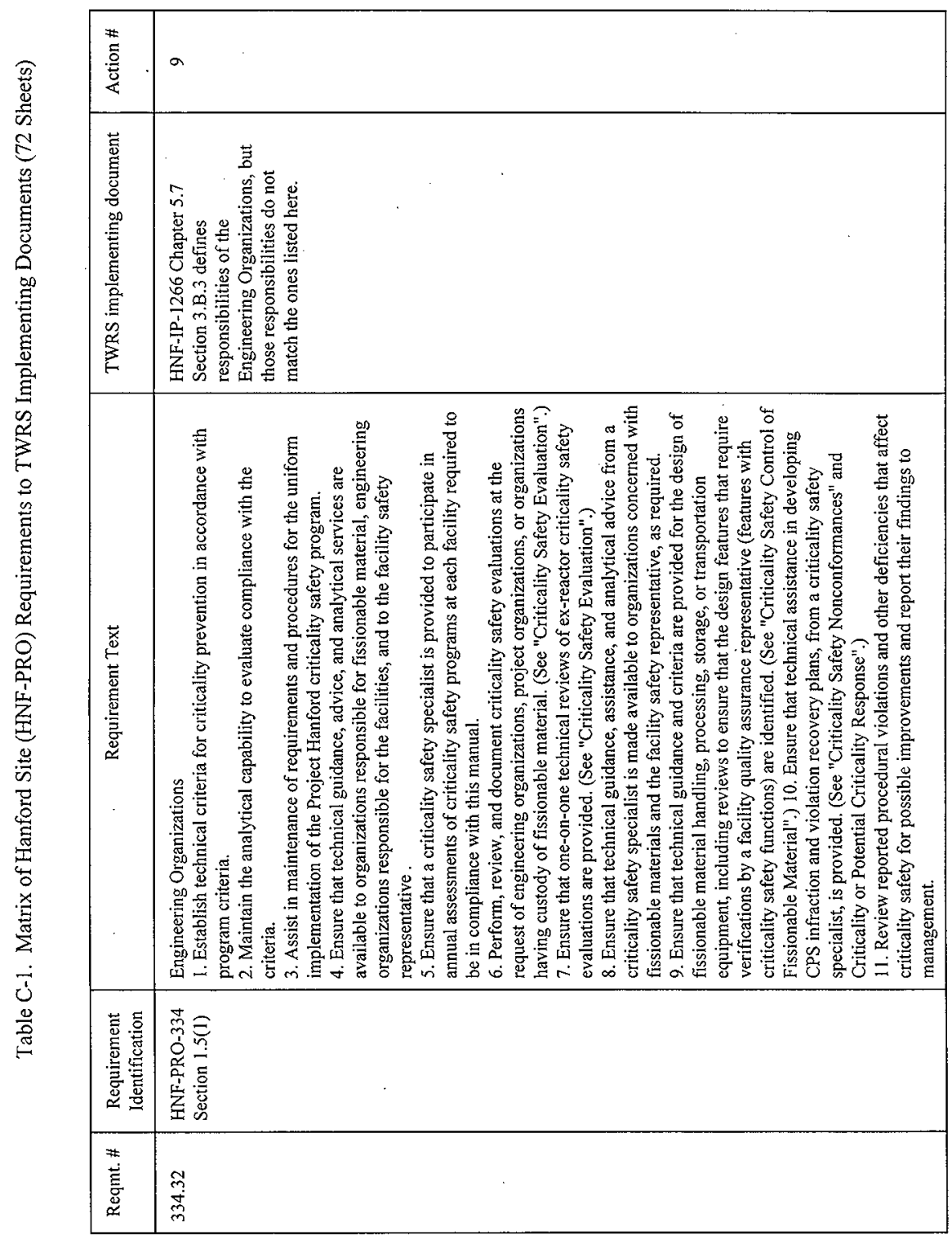

Page C-9 
TWR-3721 Rev. 0

\begin{tabular}{|c|c|c|c|c|c|c|c|c|c|c|c|}
\hline 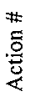 & \multicolumn{5}{|c|}{$a$} & & & & & & \\
\hline 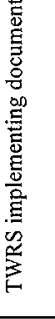 & \multicolumn{5}{|c|}{ 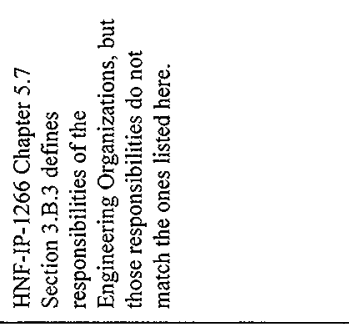 } & \multicolumn{2}{|c|}{ 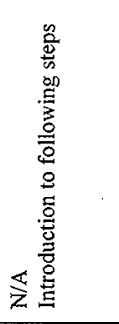 } & 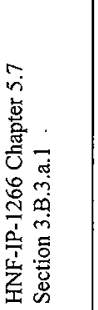 & \multicolumn{2}{|l|}{ 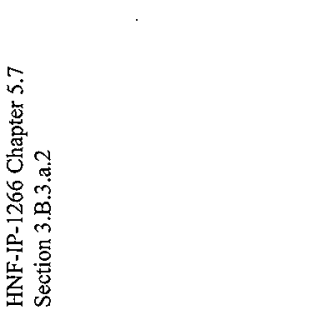 } & 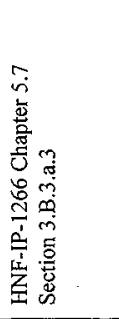 \\
\hline 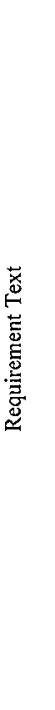 & 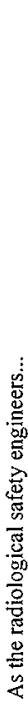 & 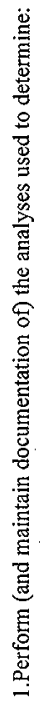 & 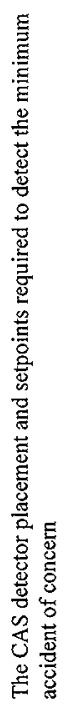 & 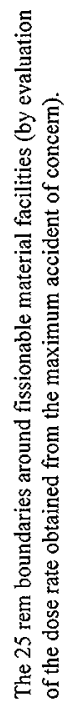 & 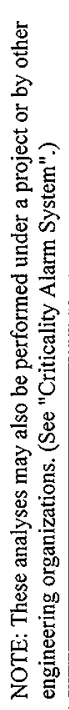 & 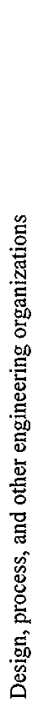 & 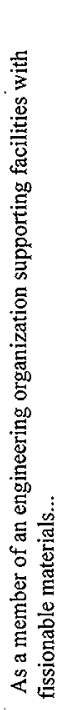 & 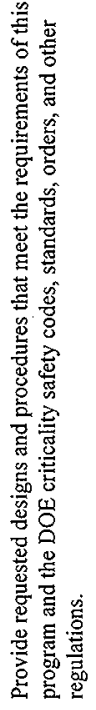 & 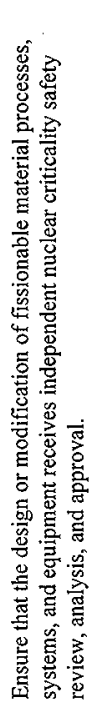 & 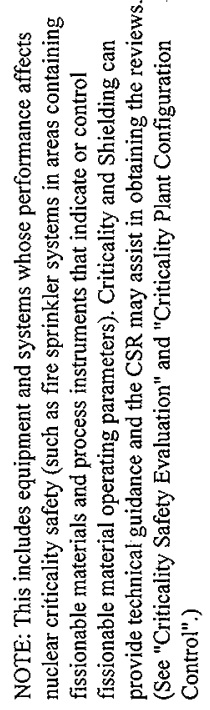 & 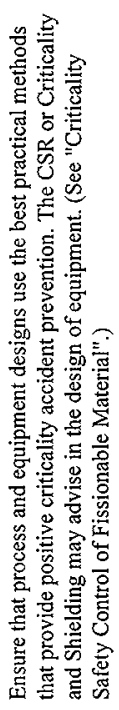 \\
\hline 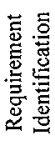 & 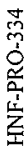 & & & & & 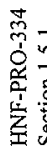 & & 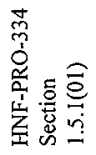 & 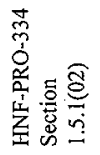 & & 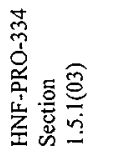 \\
\hline $\begin{array}{l}\text { ZI } \\
\stackrel{\vec{E}}{\vec{g}} \\
\approx\end{array}$ & $\stackrel{m}{\stackrel{m}{m}}$ & & & & & 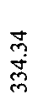 & & $\begin{array}{l}m \\
\stackrel{m}{m} \\
\text { ले }\end{array}$ & $\begin{array}{l}\stackrel{0}{m} \\
\vec{m} \\
\text { mे }\end{array}$ & & $\stackrel{\tilde{m}}{\tilde{m}}$ \\
\hline
\end{tabular}

Page C-10 
TWR-3721 Rev. 0

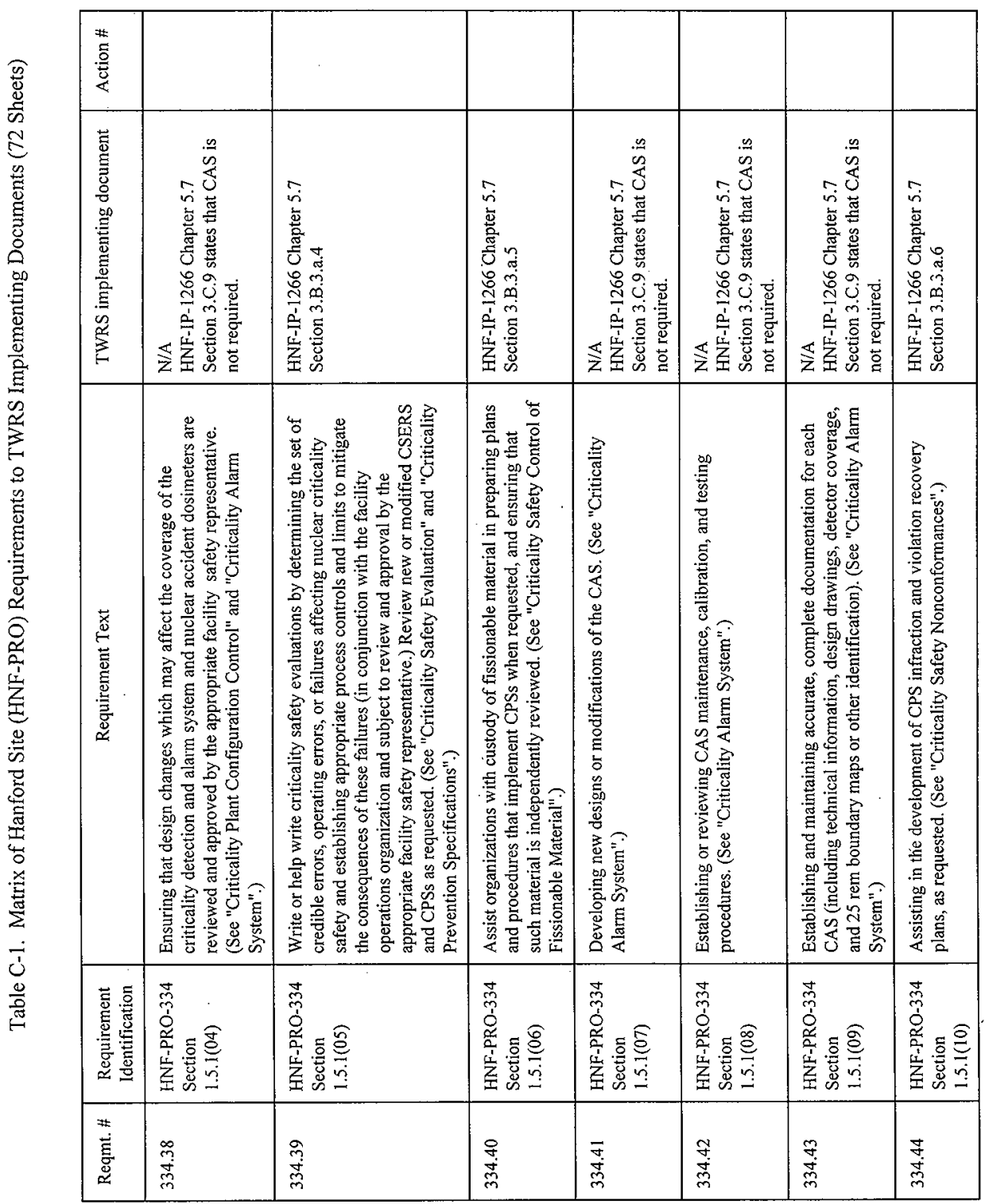


TWR-3721 Rev. 0

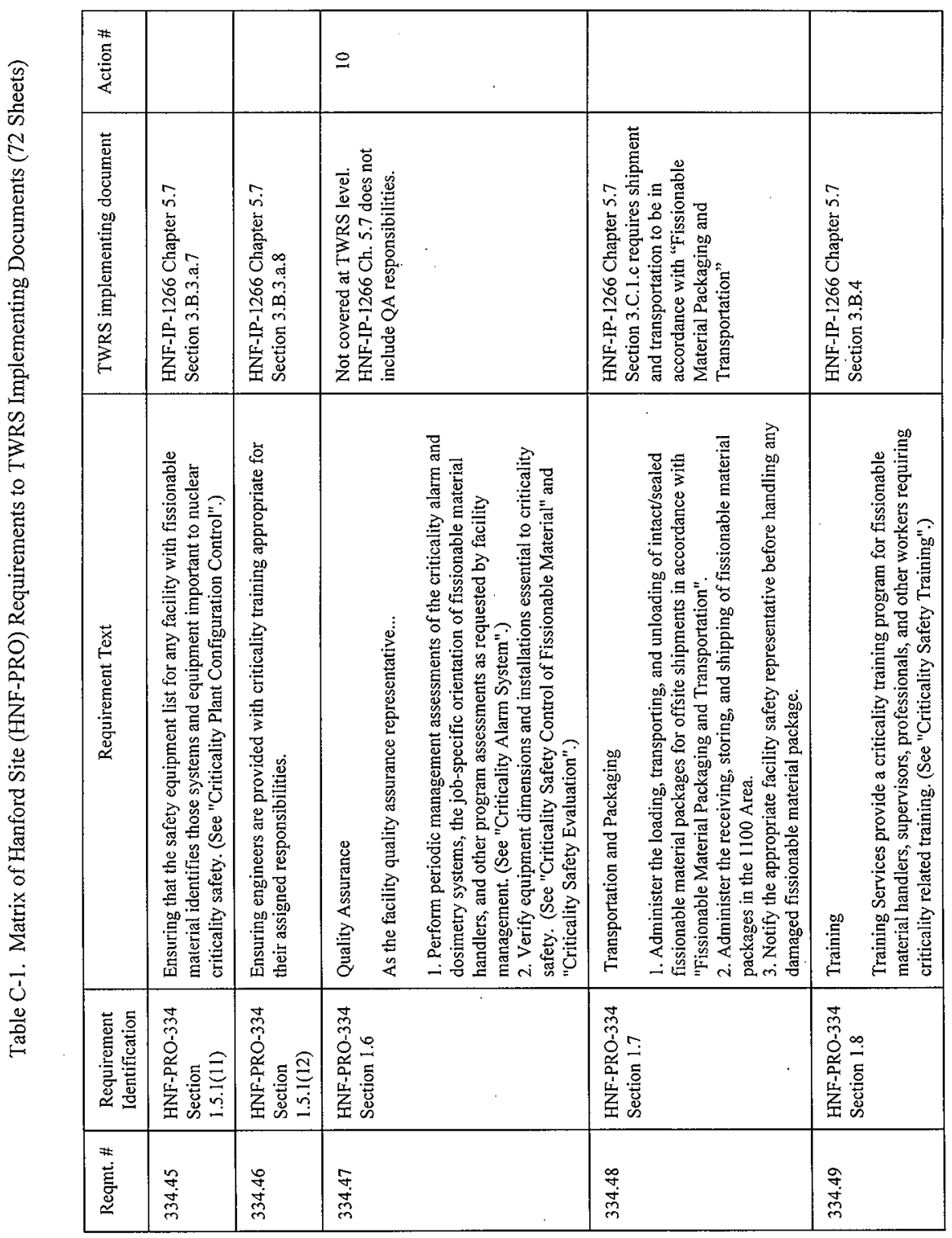

Page C-12 


\begin{tabular}{|c|c|c|c|c|}
\hline 装 & & & & \\
\hline 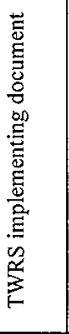 & 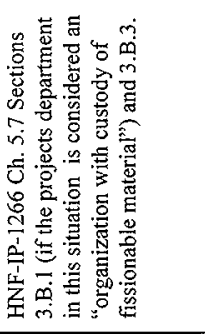 & 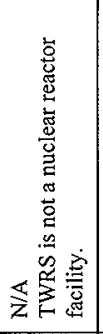 & 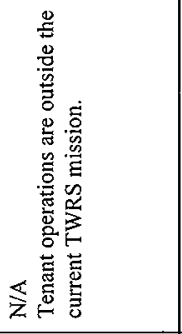 & 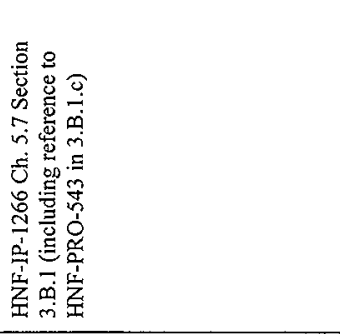 \\
\hline 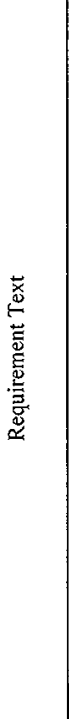 & 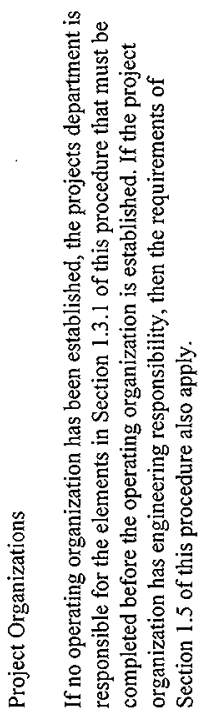 & 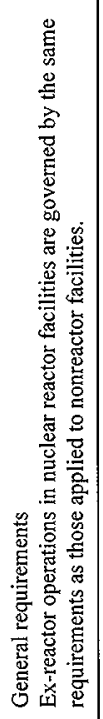 & 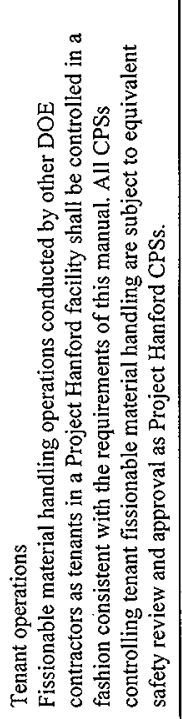 & 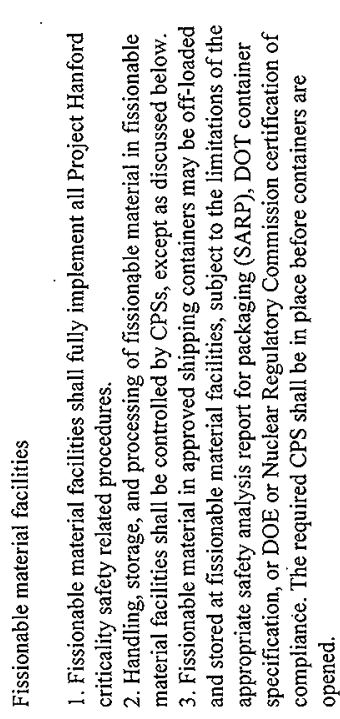 \\
\hline 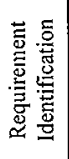 & 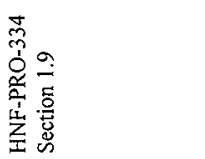 & 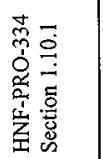 & 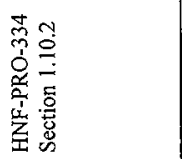 & 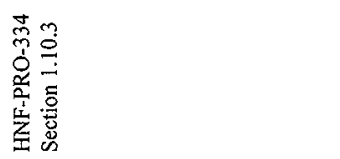 \\
\hline $\begin{array}{l}\text { \#t } \\
\text { 䓂 } \\
\text { }\end{array}$ & $\begin{array}{l}\stackrel{8}{n} \\
\text { 总 }\end{array}$ & $\begin{array}{l}\overline{\bar{n}} \\
\bar{m} \\
\text { ne }\end{array}$ & $\begin{array}{l}\tilde{n} \\
\tilde{m} \\
\tilde{m}\end{array}$ & 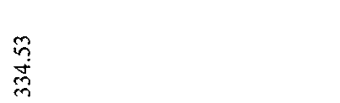 \\
\hline
\end{tabular}


TWR-3721 Rev. 0

\begin{tabular}{|c|c|c|c|c|c|c|}
\hline $\begin{array}{l}\# \\
\text { \#总 } \\
\text { 芳 }\end{array}$ & & & $=$ & & & \\
\hline 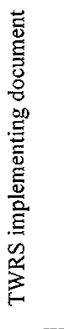 & 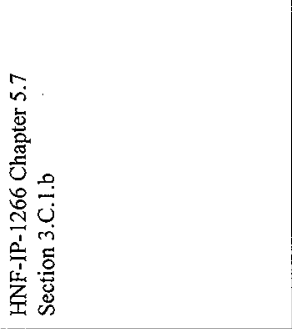 & 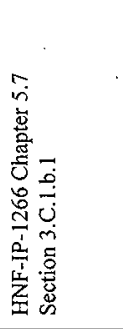 & 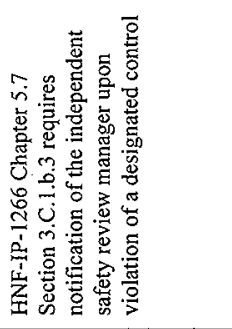 & 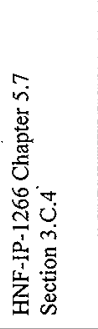 & 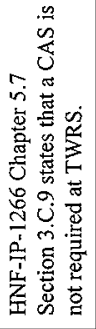 & 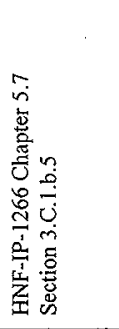 \\
\hline 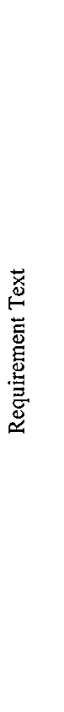 & 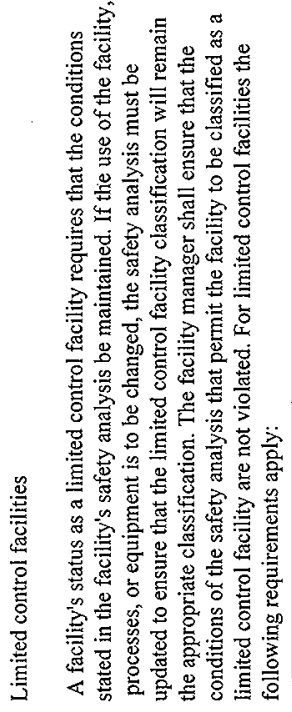 & 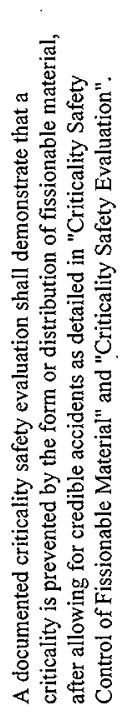 & 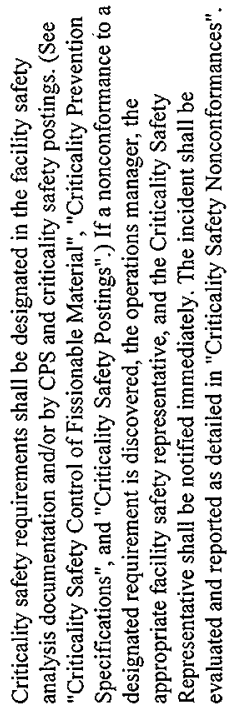 & 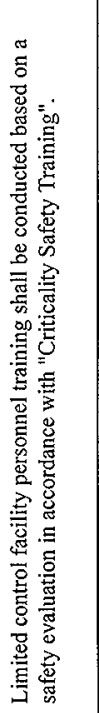 & 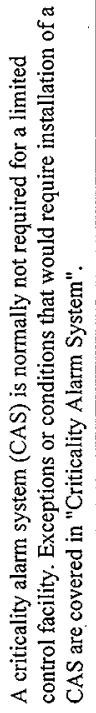 & 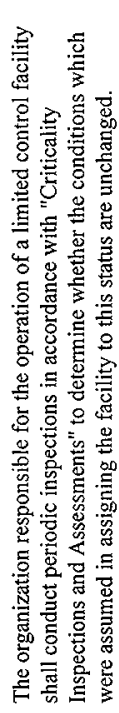 \\
\hline 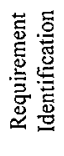 & 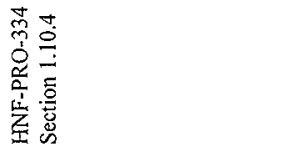 & 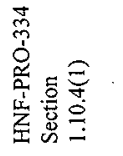 & 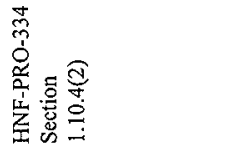 & 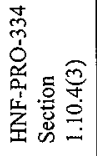 & 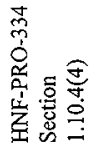 & 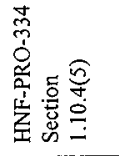 \\
\hline 葛 & $\begin{array}{l}\vec{v} \\
\tilde{m} \\
\text { n}\end{array}$ & $\begin{array}{l}n \\
\stackrel{n}{m}\end{array}$ & $\begin{array}{l}\stackrel{\circ}{n} \\
\stackrel{5}{n}\end{array}$ & $\begin{array}{l}\tilde{n} \\
\tilde{m} \\
\tilde{m}\end{array}$ & $\begin{array}{l}\infty \\
\stackrel{n}{m} \\
\stackrel{m}{m}\end{array}$ & $\begin{array}{l}\vec{n} \\
\dot{m} \\
\text { man }\end{array}$ \\
\hline
\end{tabular}

Page C-14 
TWR-3721 Rev. 0

\begin{tabular}{|c|c|c|c|c|c|c|c|c|}
\hline 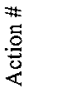 & & & & & & & & \\
\hline 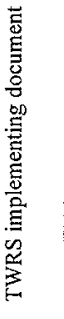 & 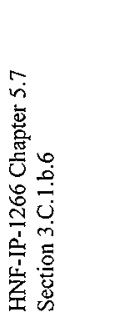 & 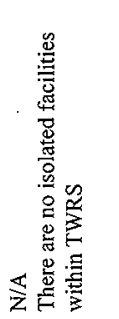 & 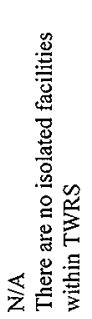 & 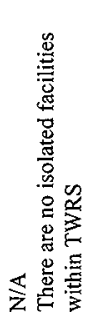 & 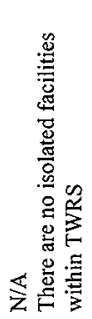 & 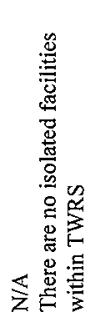 & 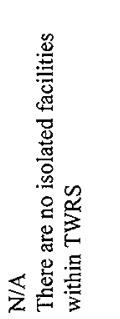 & 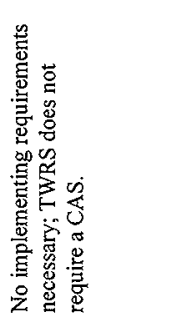 \\
\hline 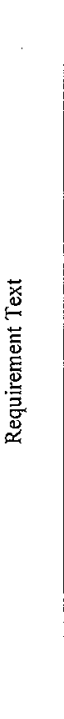 & 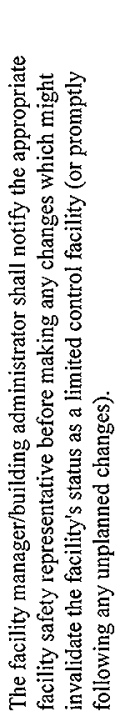 & 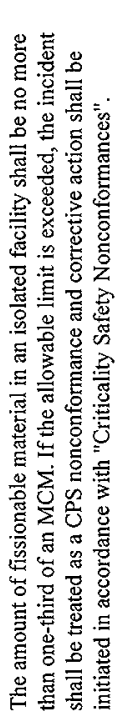 & 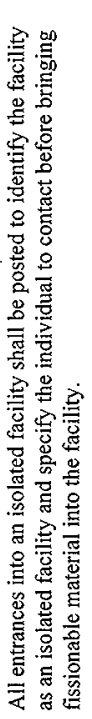 & 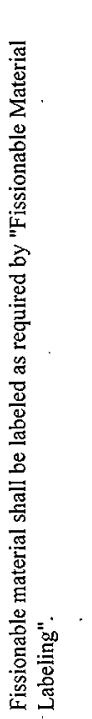 & 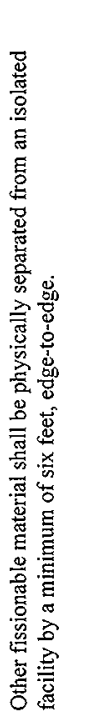 & 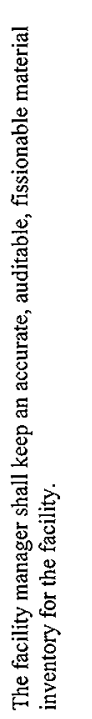 & 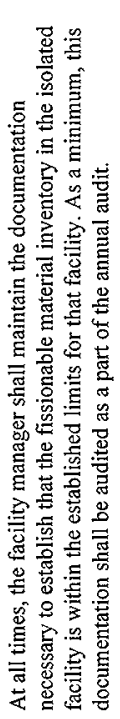 & 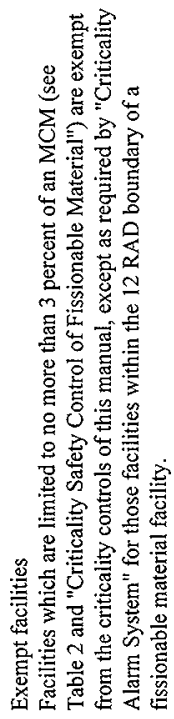 \\
\hline 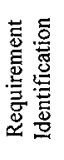 & 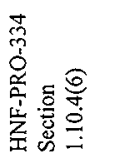 & 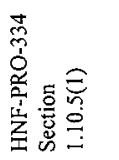 & 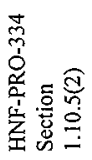 & 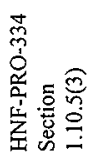 & 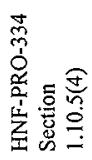 & 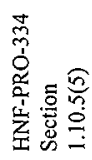 & 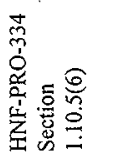 & 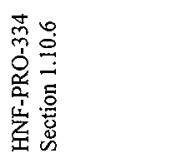 \\
\hline 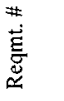 & $\begin{array}{l}8 \\
\text { ज } \\
\text { m }\end{array}$ & $\begin{array}{l}\vec{b} \\
\vec{m}\end{array}$ & 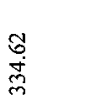 & $\begin{array}{l}\hat{6} \\
\stackrel{\mathrm{m}}{\mathrm{m}}\end{array}$ & 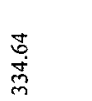 & 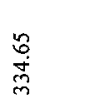 & $\begin{array}{l}\stackrel{8}{0} \\
\text { 品 }\end{array}$ & 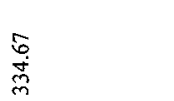 \\
\hline
\end{tabular}


TWR-3721 Rev. 0

\begin{tabular}{|c|c|c|c|c|}
\hline 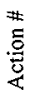 & & & & \\
\hline 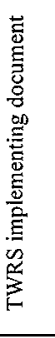 & 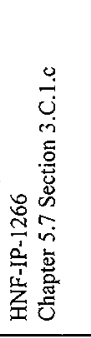 & 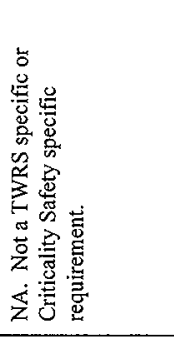 & 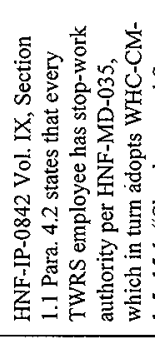 & 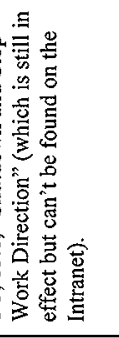 \\
\hline 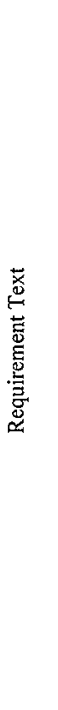 & 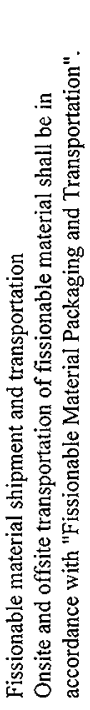 & 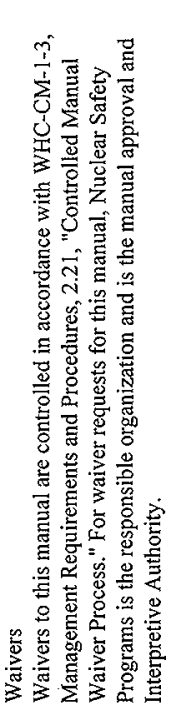 & \multicolumn{2}{|c|}{ 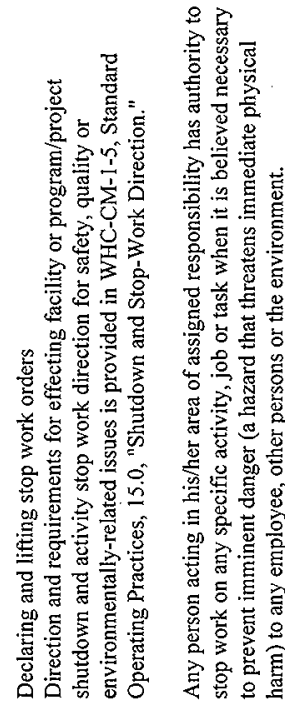 } \\
\hline 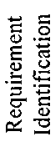 & 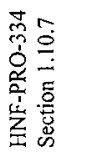 & 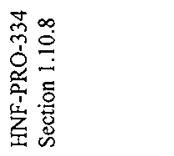 & 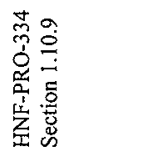 & \\
\hline $\begin{array}{l}\text { \# } \\
\text { 芯 } \\
\text { 导 }\end{array}$ & $\begin{array}{l}\infty \\
\stackrel{\infty}{\circ} \\
\stackrel{+}{m}\end{array}$ & $\begin{array}{l}\stackrel{g}{0} \\
\stackrel{+}{ \pm} \\
\text { ma }\end{array}$ & 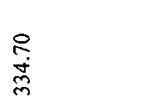 & \\
\hline
\end{tabular}

'Page C-16 
TWR-3721 Rev. 0

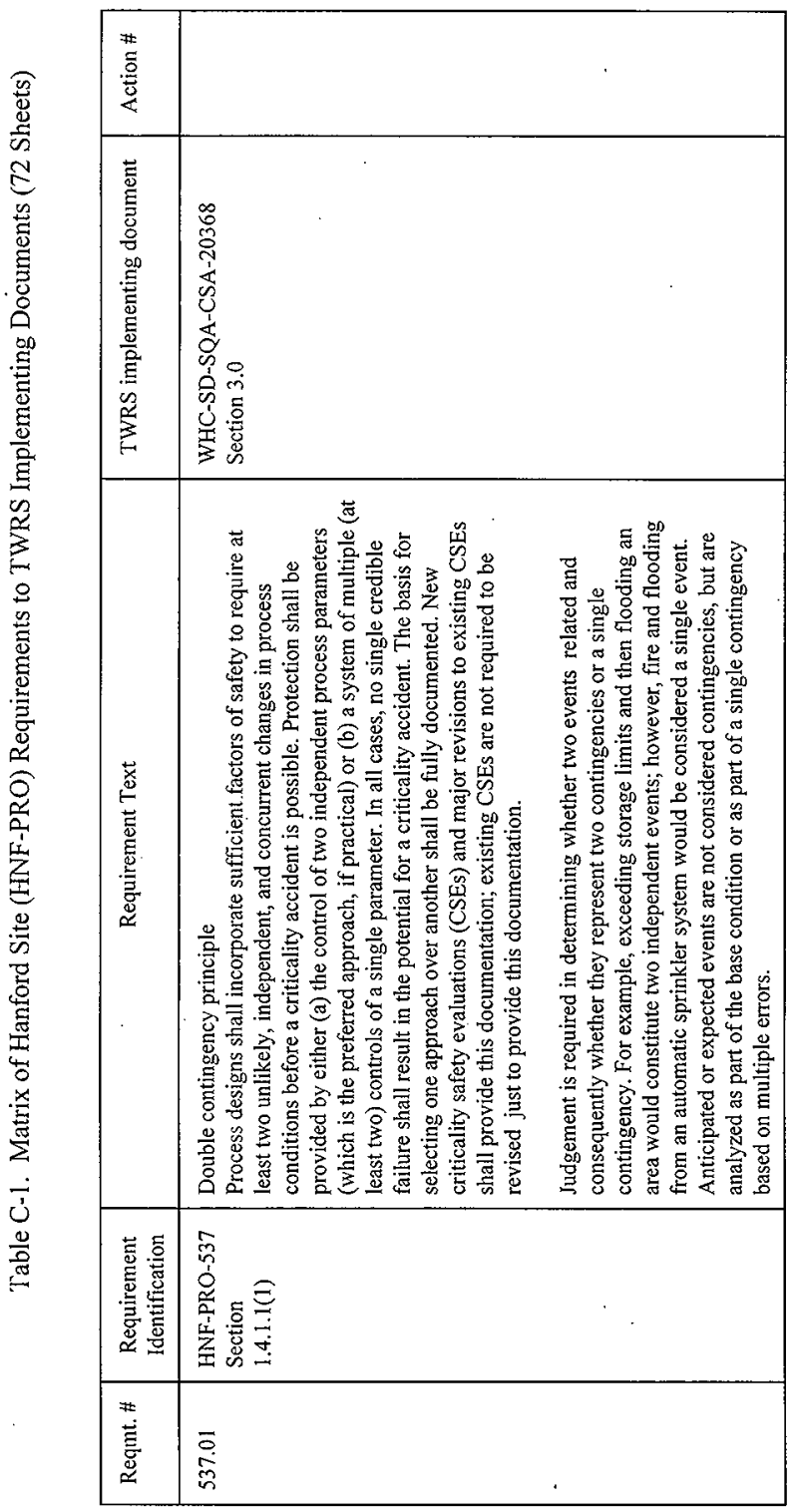

Page C-17 
TWR-3721 Rev. 0

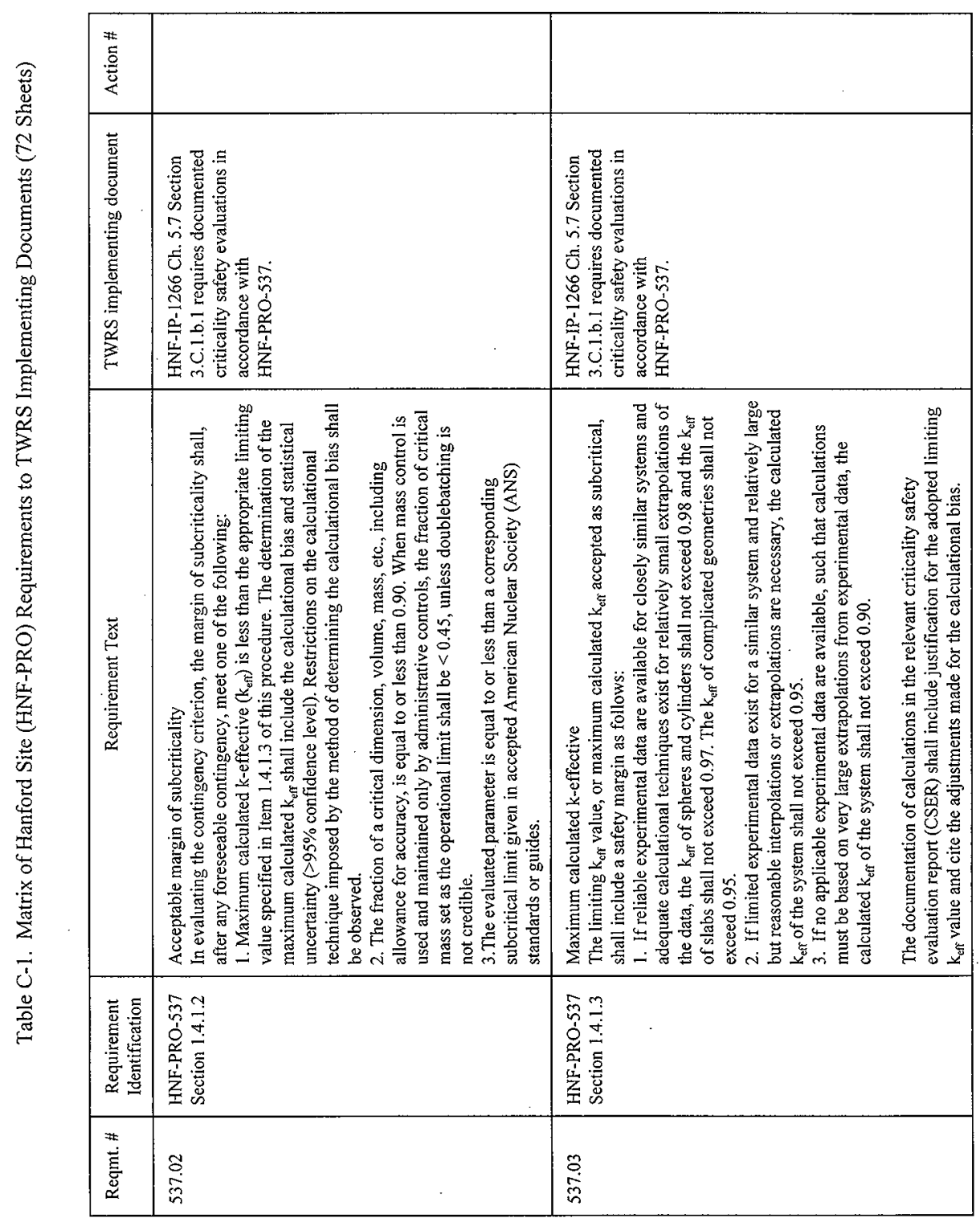

Page C-18 
TWR-3721 Rev. 0

\begin{tabular}{|c|c|c|c|c|c|c|}
\hline 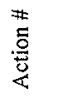 & & & & \multicolumn{3}{|l|}{$\cong$} \\
\hline 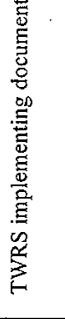 & \multicolumn{3}{|l|}{ 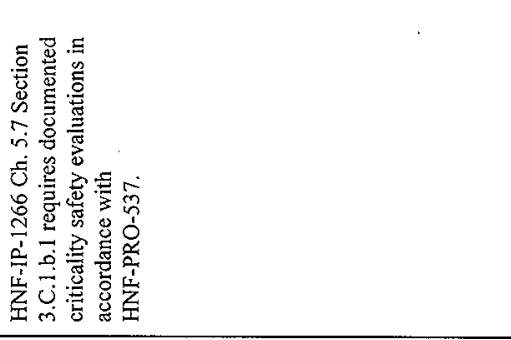 } & \multicolumn{3}{|l|}{ 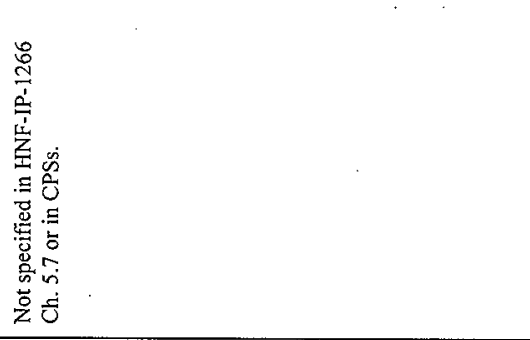 } \\
\hline 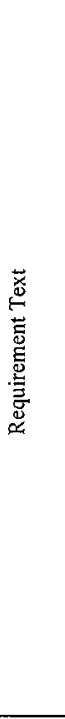 & 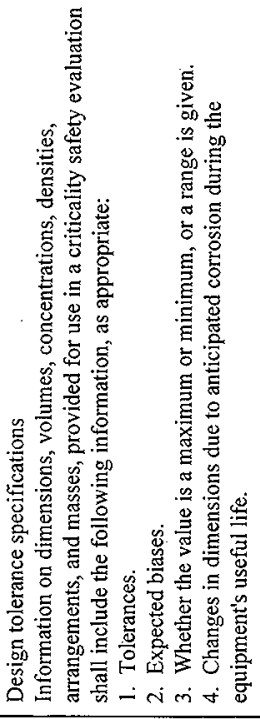 & 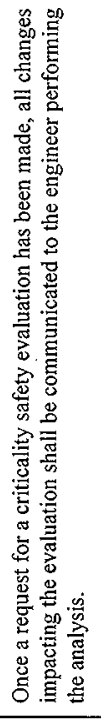 & 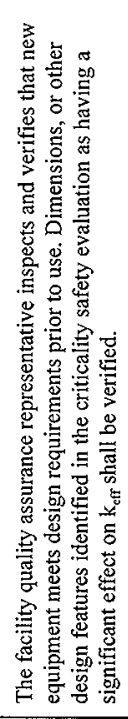 & 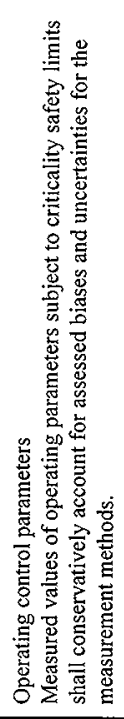 & 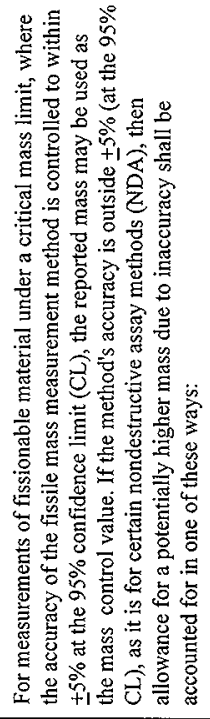 & 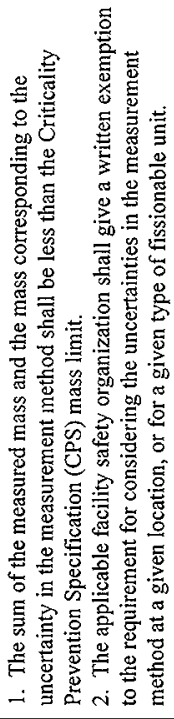 \\
\hline 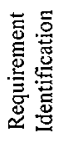 & 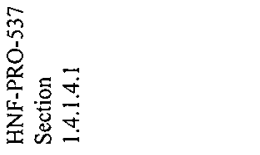 & & & 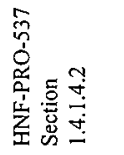 & - & \\
\hline 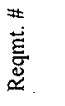 & $\begin{array}{l}\stackrel{+}{0} \\
\frac{\sigma}{n}\end{array}$ & & & $\frac{\mathscr{c}}{\tilde{n}}$ & & \\
\hline
\end{tabular}

Page C-19 
TWR-3721 Rev. 0

\begin{tabular}{|c|c|c|c|}
\hline 莣 & 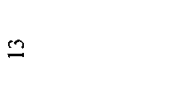 & & \\
\hline 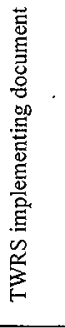 & 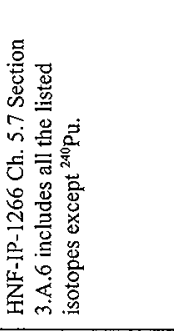 & 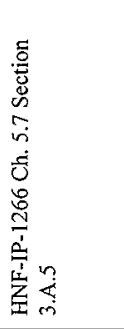 & 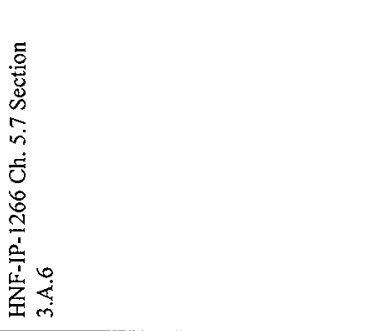 \\
\hline 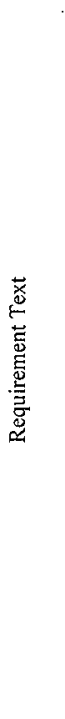 & 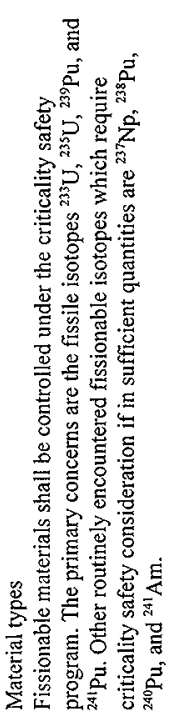 & 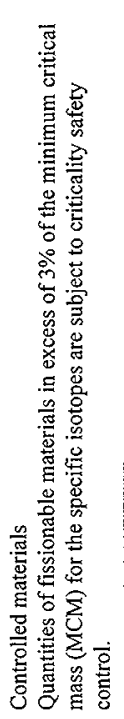 & 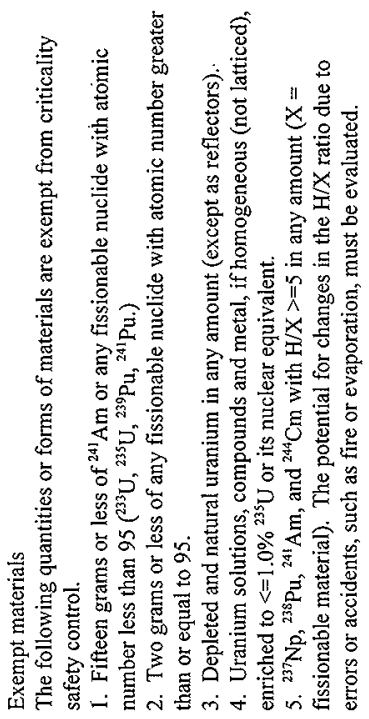 \\
\hline 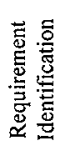 & 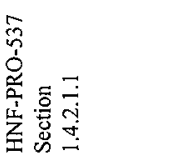 & 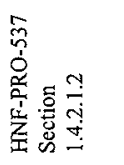 & 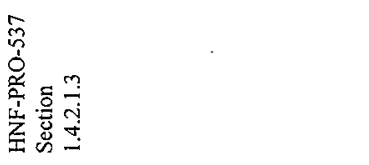 \\
\hline 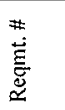 & 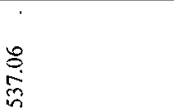 & $\frac{5}{6}$ & $\begin{array}{l}\infty \\
\stackrel{8}{5} \\
\stackrel{3}{\Omega}\end{array}$ \\
\hline
\end{tabular}

Page C-20 
TWR-3721 Rev. 0

\begin{tabular}{|c|c|c|c|c|c|}
\hline 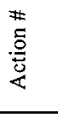 & & & & & \\
\hline 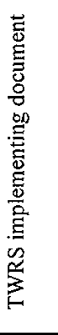 & 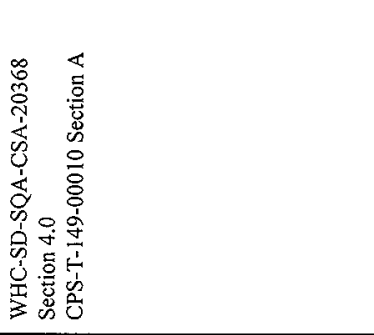 & 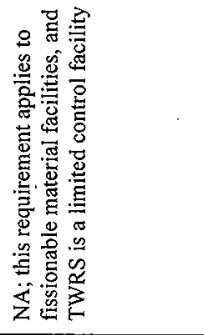 & 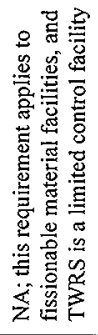 & 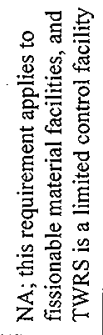 & 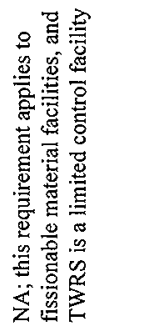 \\
\hline 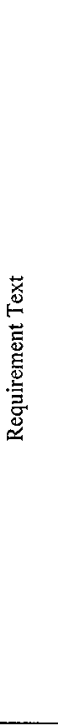 & 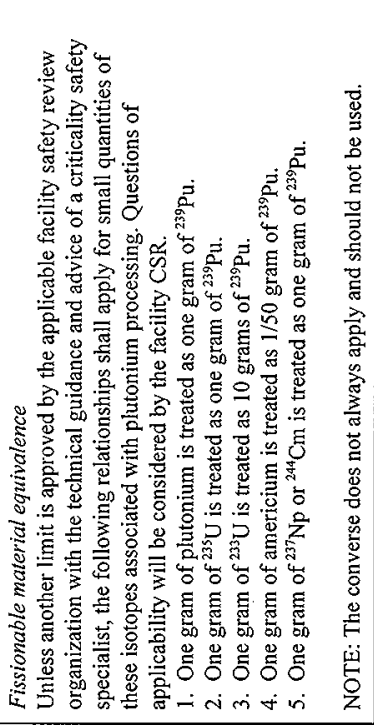 & 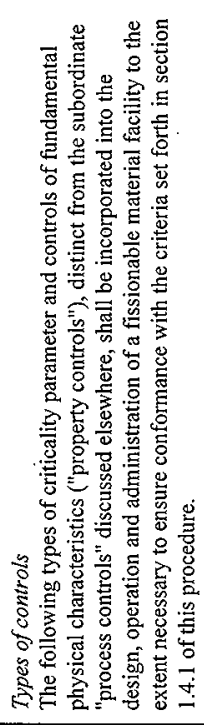 & 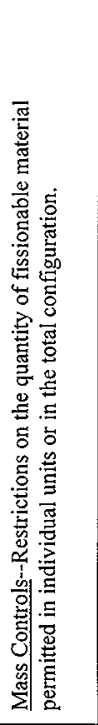 & 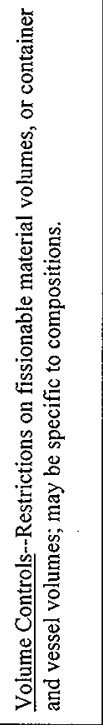 & 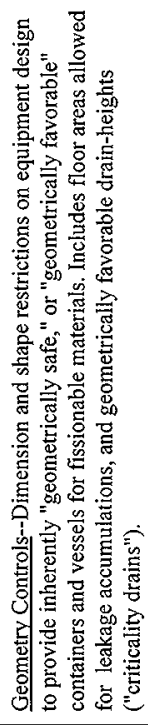 \\
\hline 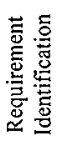 & 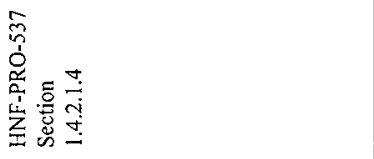 & 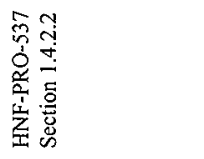 & 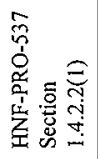 & 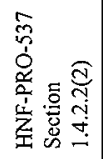 & 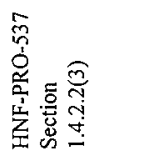 \\
\hline 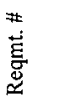 & 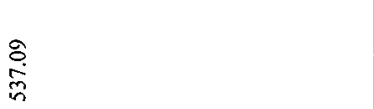 & $\underset{\hat{n}}{\stackrel{9}{*}}$ & $\underset{\infty}{\vec{n}}$ & $\underset{i n}{\stackrel{7}{n}}$ & $\stackrel{m}{\vec{n}}$ \\
\hline
\end{tabular}


TWR-3721 Rev. 0

\begin{tabular}{|c|c|c|c|c|c|c|c|c|}
\hline 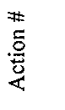 & & & & & & & & \\
\hline 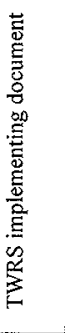 & 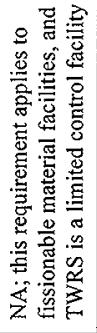 & 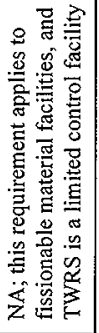 & 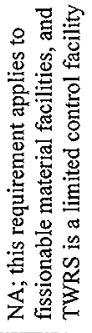 & 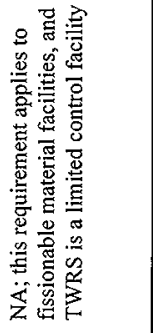 & 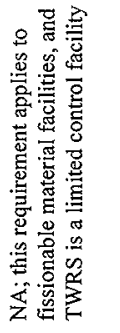 & 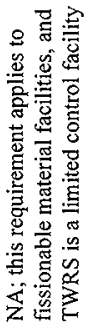 & 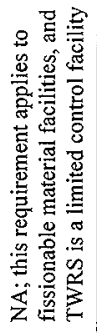 & 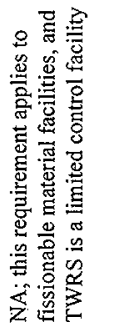 \\
\hline 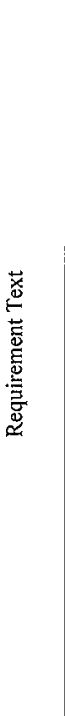 & 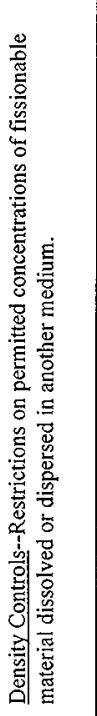 & 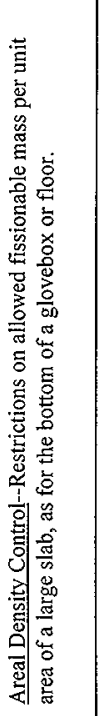 & 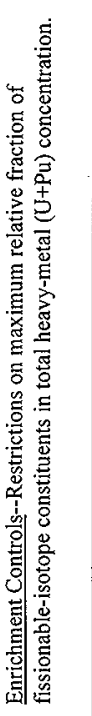 & 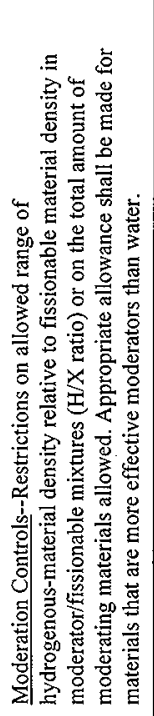 & 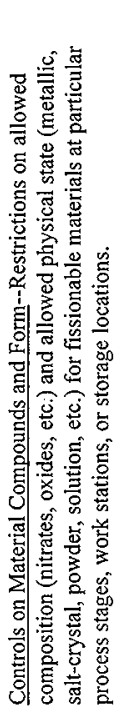 & 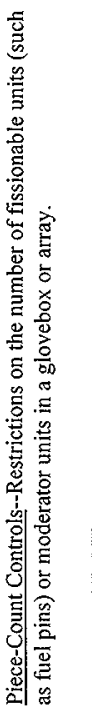 & 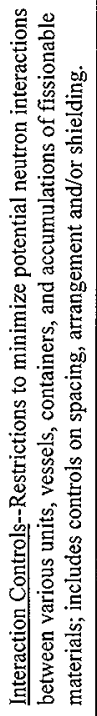 & 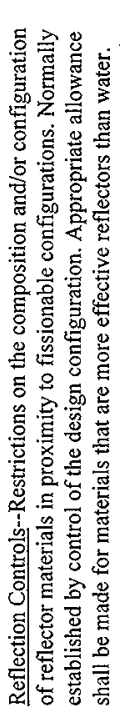 \\
\hline 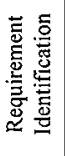 & 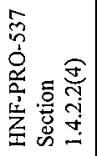 & 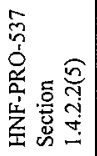 & 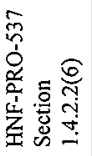 & 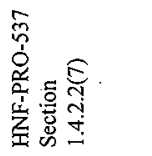 & 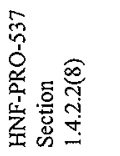 & 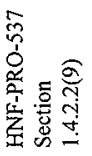 & 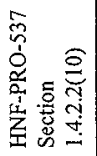 & 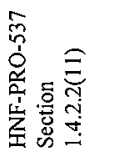 \\
\hline 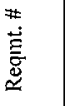 & $\frac{ \pm}{n}$ & $\frac{n}{n} \frac{n}{n}$ & $\frac{0}{n}$ & $\frac{n}{n}$ & $\frac{\infty}{n}$ & $\frac{a}{i}$ & 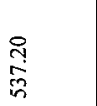 & 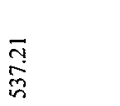 \\
\hline
\end{tabular}


TWR-3721 Rev. 0

\begin{tabular}{|c|c|c|c|c|c|}
\hline 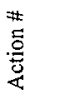 & & & & & \\
\hline 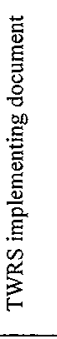 & 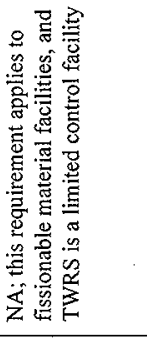 & 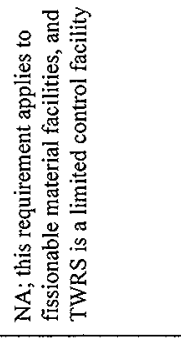 & 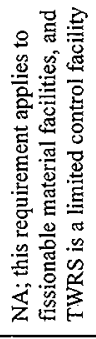 & 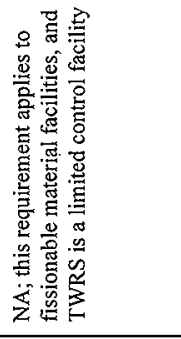 & 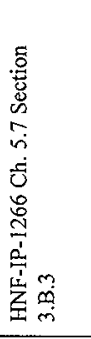 \\
\hline 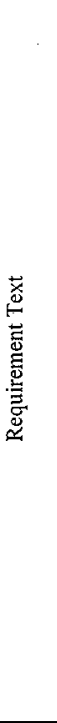 & 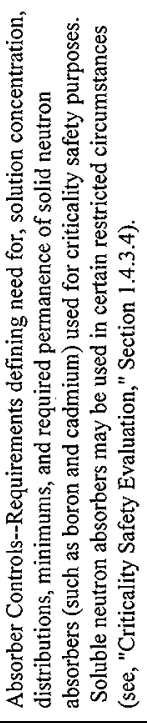 & 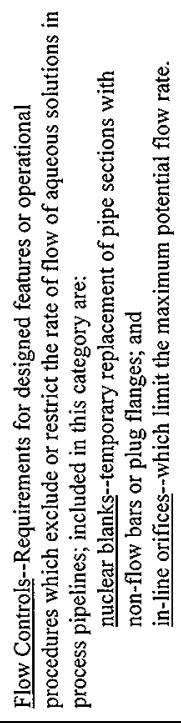 & 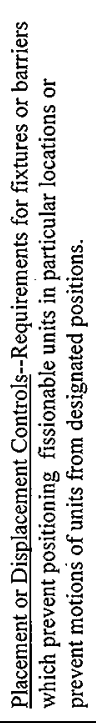 & 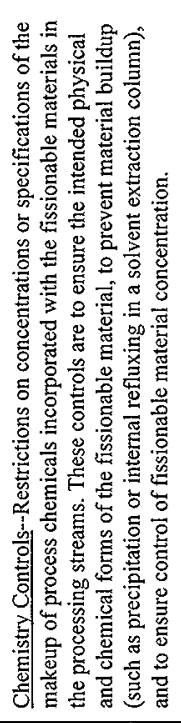 & 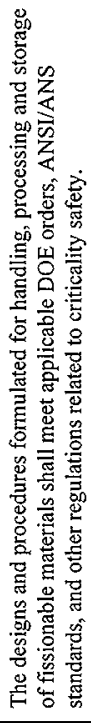 \\
\hline 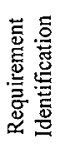 & 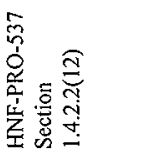 & 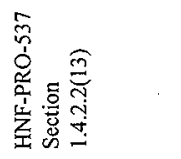 & 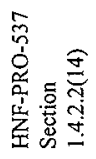 & 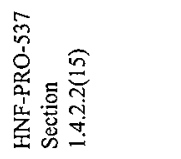 & 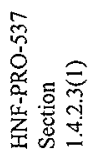 \\
\hline 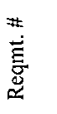 & 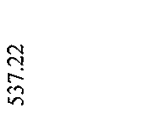 & 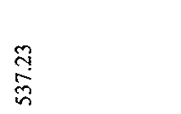 & $\underset{\substack{i \\
i n n}}{\mathbb{N}}$ & $\underset{\substack{n \\
n}}{\stackrel{n}{n}}$ & 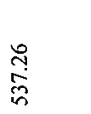 \\
\hline
\end{tabular}




\begin{tabular}{|c|c|c|c|c|}
\hline 華 & & & & \pm \\
\hline 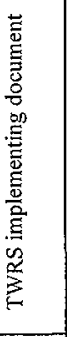 & 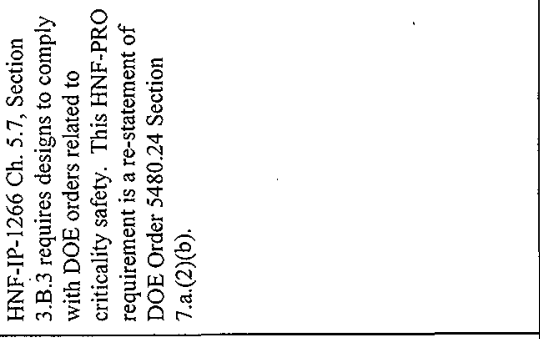 & 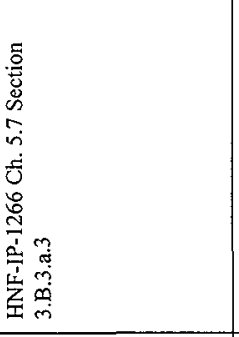 & 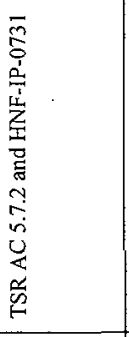 & 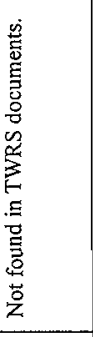 \\
\hline 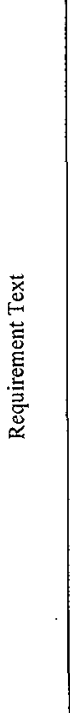 & 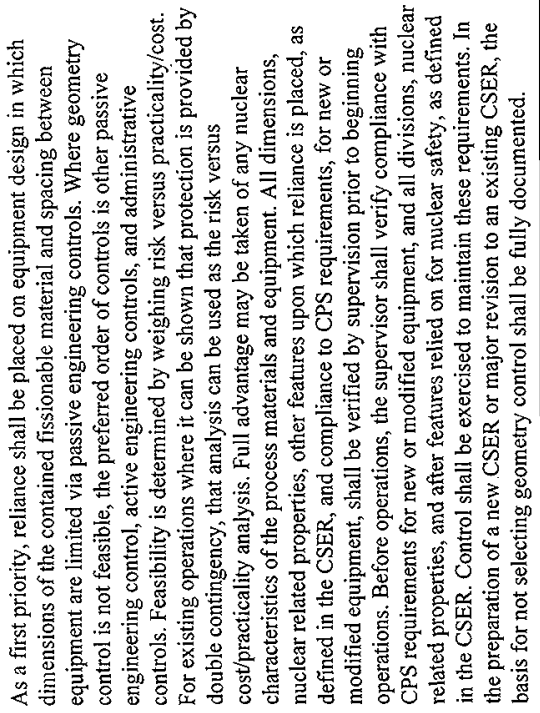 & 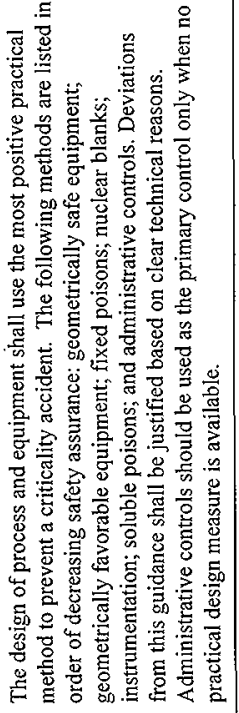 & 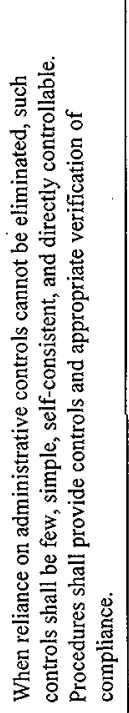 & 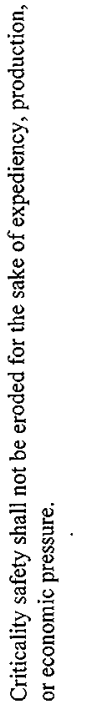 \\
\hline 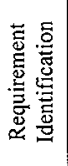 & 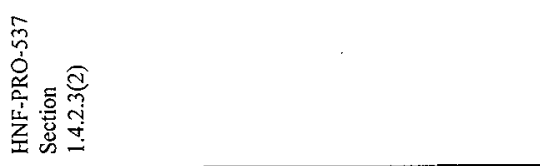 & 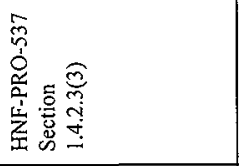 & 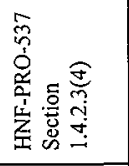 & 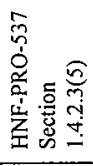 \\
\hline 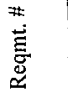 & $\underset{\stackrel{N}{n}}{\stackrel{ }{n}}$ & 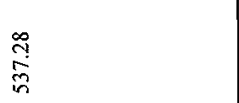 & 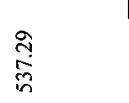 & 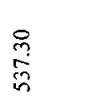 \\
\hline
\end{tabular}


TWR-3721 Rev. 0

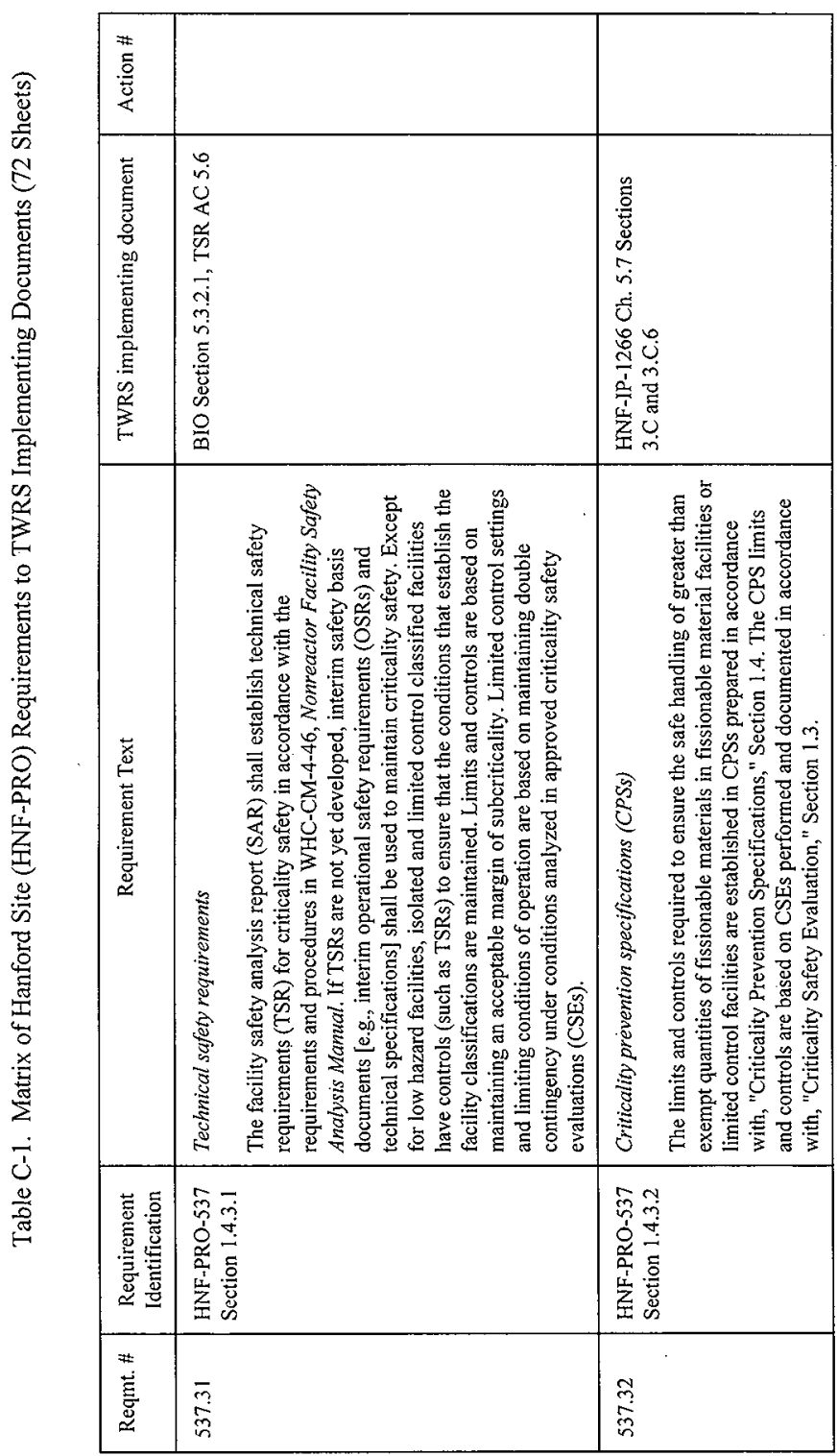

Page C-25 
TWR-3721 Rev. 0

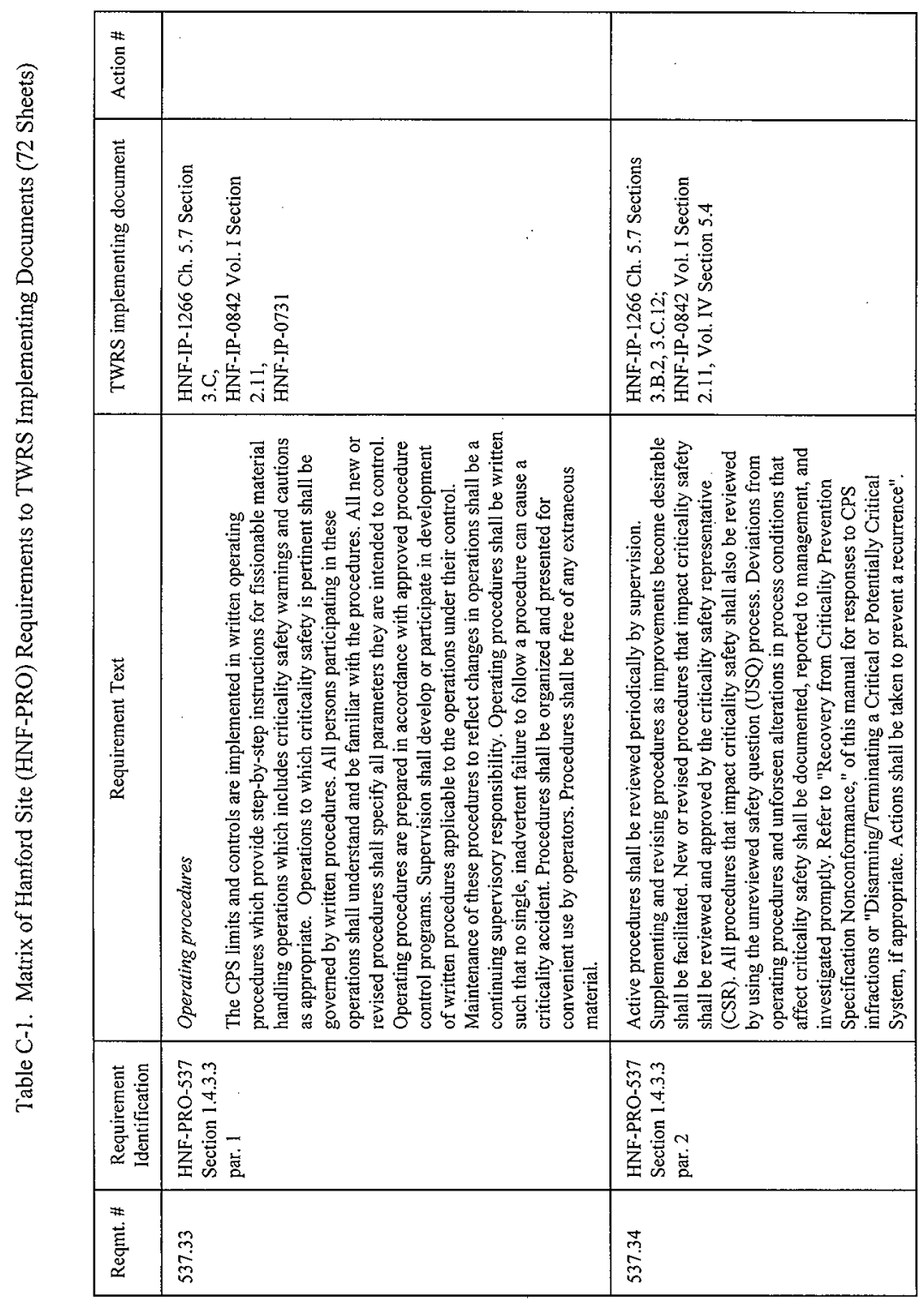

Page C-26 
TWR-3721 Rev. 0

\begin{tabular}{|c|c|c|c|c|c|}
\hline 華 & & & & & \\
\hline 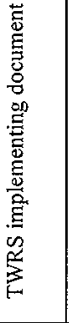 & 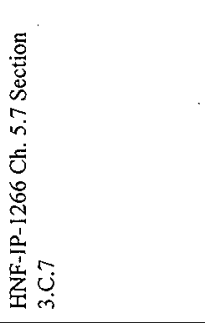 & 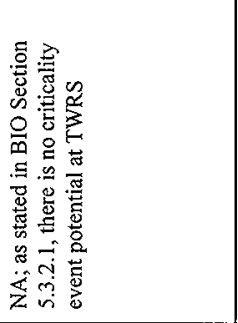 & 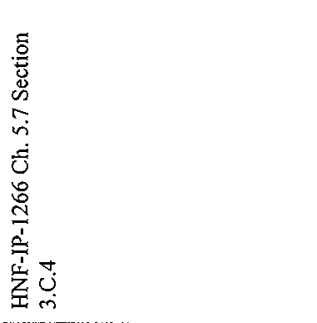 & 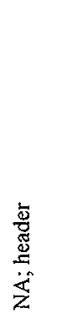 & 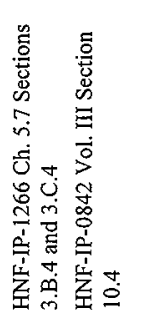 \\
\hline 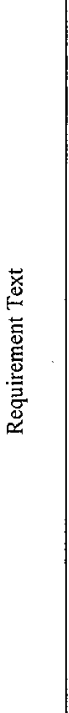 & 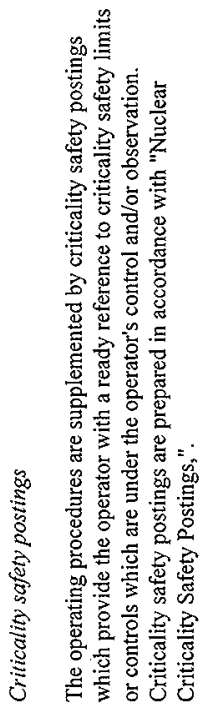 & 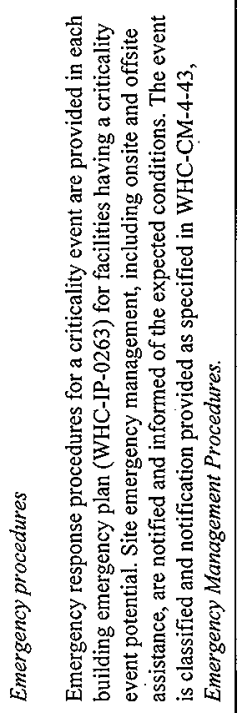 & 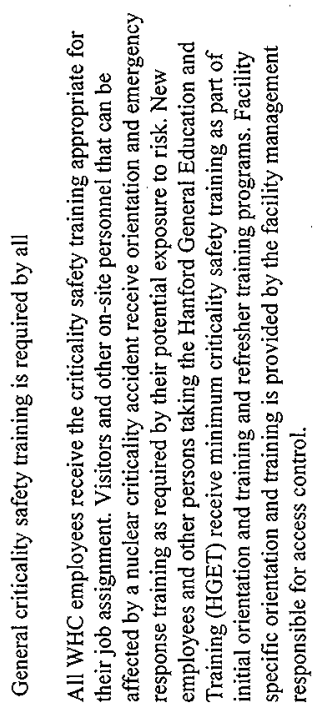 & 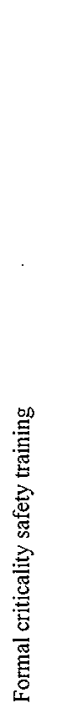 & 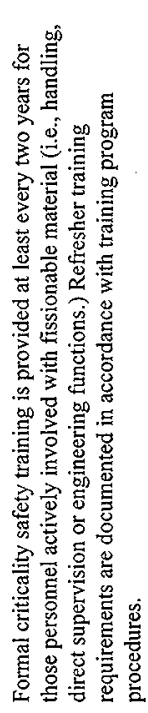 \\
\hline 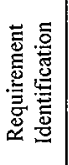 & 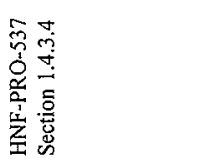 & 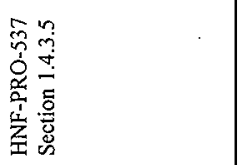 & 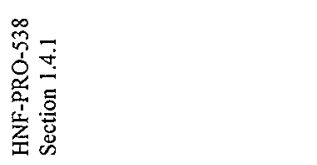 & 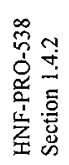 & 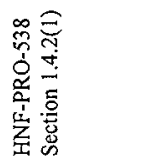 \\
\hline 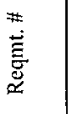 & $\stackrel{\stackrel{m}{n}}{\stackrel{n}{n}}$ & సొ? & $\begin{array}{l}\overline{0} \\
\infty \\
\tilde{n} \\
\tilde{n}\end{array}$ & $\begin{array}{l}\tilde{\delta} \\
\infty \\
0 \\
\tilde{n}\end{array}$ & \begin{tabular}{l}
$\tilde{c}$ \\
$\infty$ \\
\hdashline \\
$\tilde{n}$
\end{tabular} \\
\hline
\end{tabular}

Page C-27 
TWR-3721 Rev. 0

\begin{tabular}{|c|c|c|c|c|c|c|}
\hline \# & & & $n$ & \multicolumn{3}{|l|}{$\stackrel{\circ}{\circ}$} \\
\hline 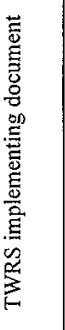 & 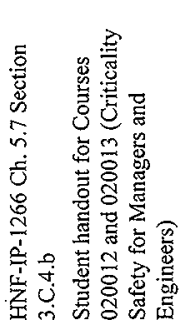 & 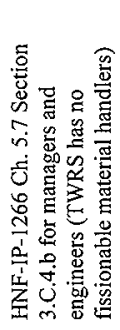 & 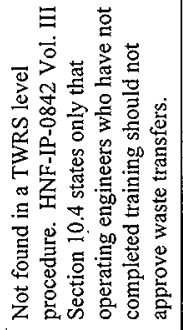 & \multicolumn{3}{|c|}{ 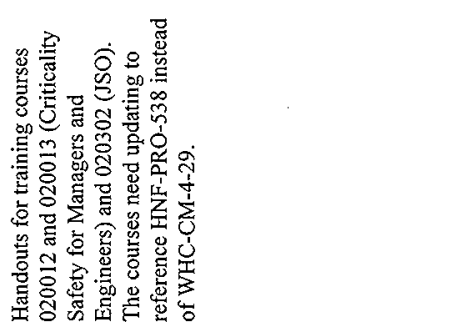 } \\
\hline 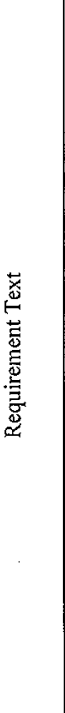 & 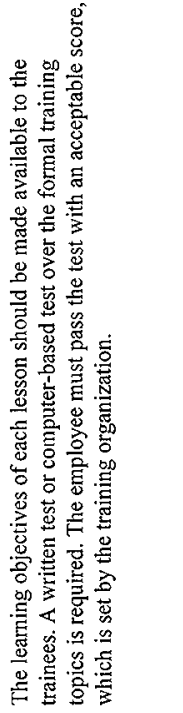 & 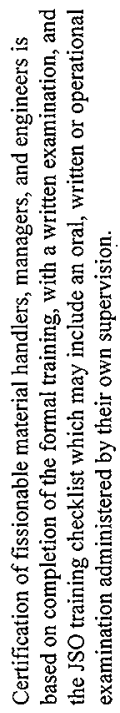 & 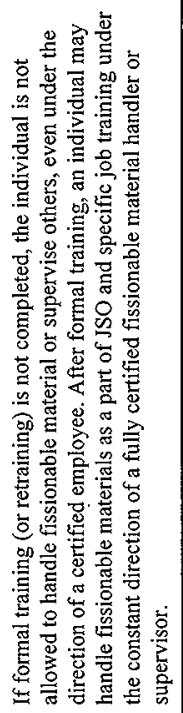 & 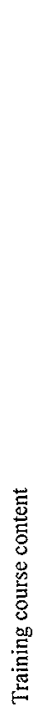 & 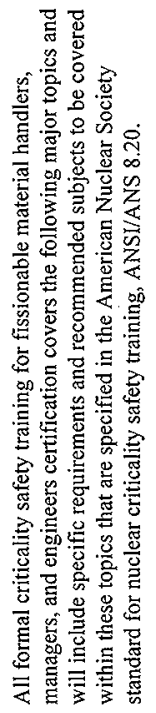 & 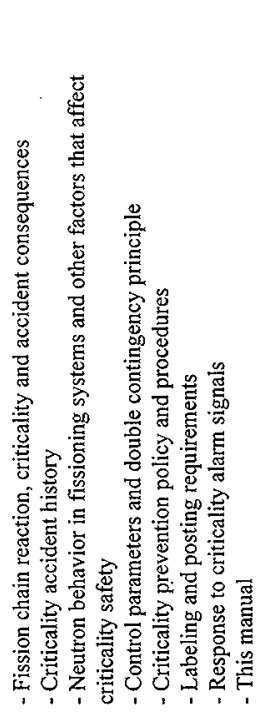 \\
\hline 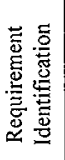 & 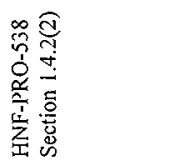 & 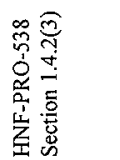 & 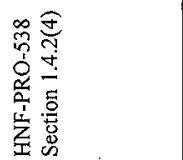 & 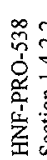 & & \\
\hline 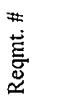 & $\begin{array}{l} \pm \\
0 \\
\infty \\
n \\
n\end{array}$ & $\begin{array}{l}\stackrel{n}{0} \\
\infty \\
\infty \\
n \\
n\end{array}$ & $\begin{array}{l}\mathscr{0} \\
\infty \\
\infty \\
0 \\
n\end{array}$ & $\begin{array}{l}5 \\
\infty \\
\infty \\
\infty \\
n\end{array}$ & & \\
\hline
\end{tabular}


TWR-3721 Rev. 0

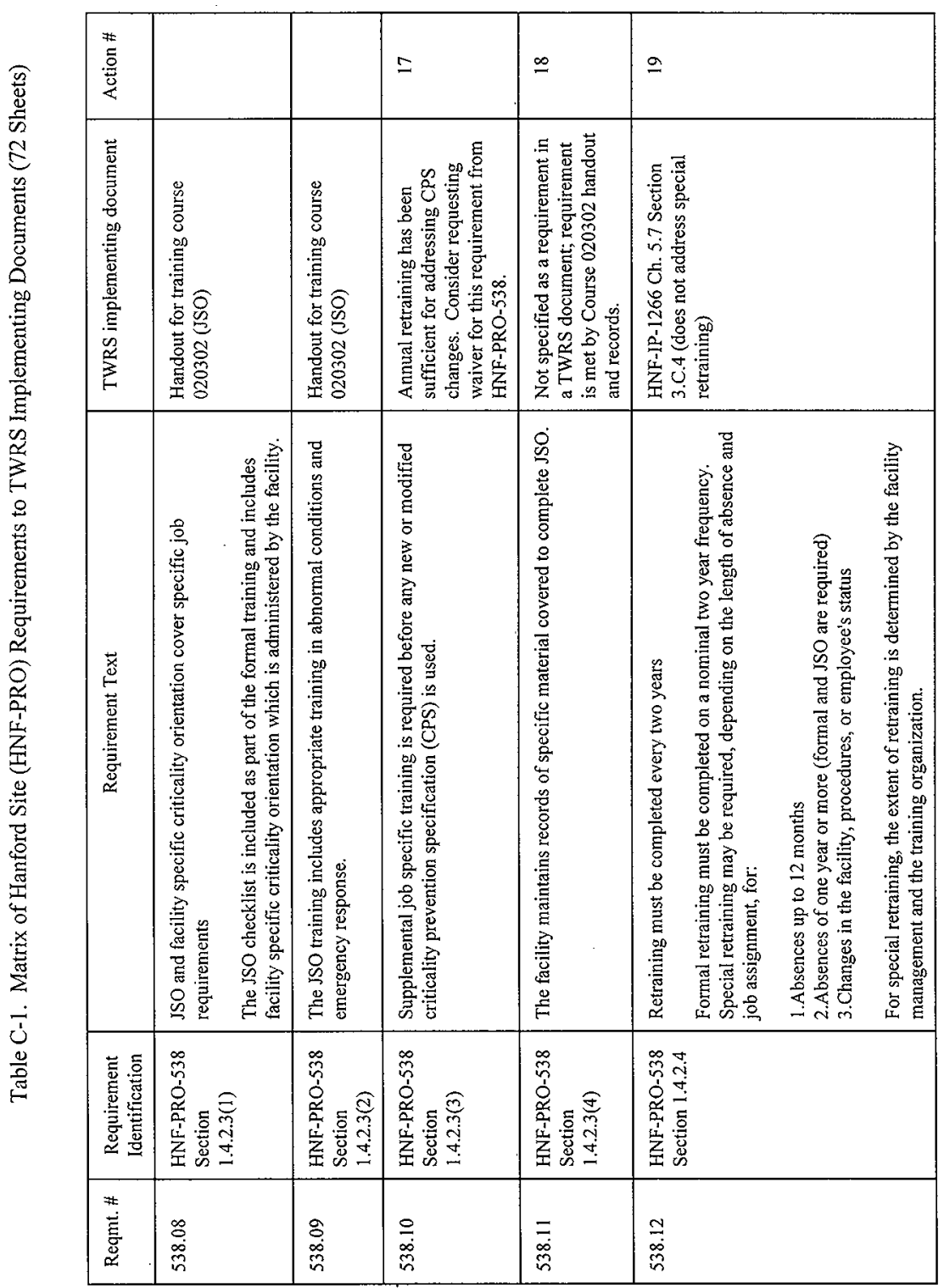

Page C-29 
TWR-3721 Rev. 0

\begin{tabular}{|c|c|c|c|}
\hline 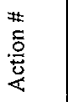 & & \multicolumn{2}{|l|}{ ి. } \\
\hline 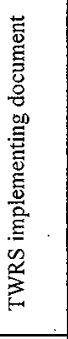 & 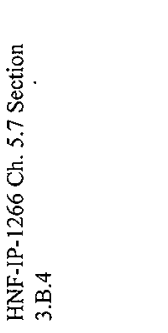 & \multicolumn{2}{|c|}{ 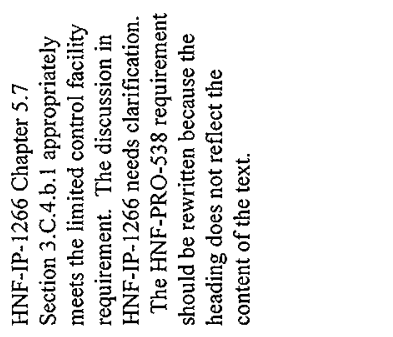 } \\
\hline 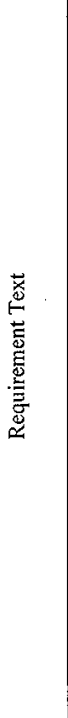 & 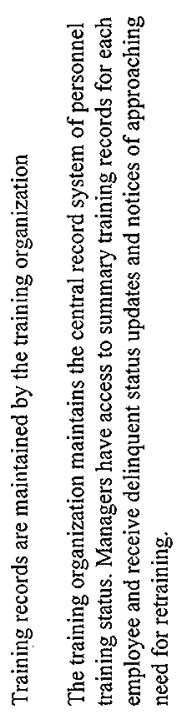 & 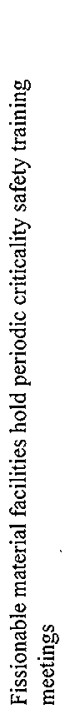 & 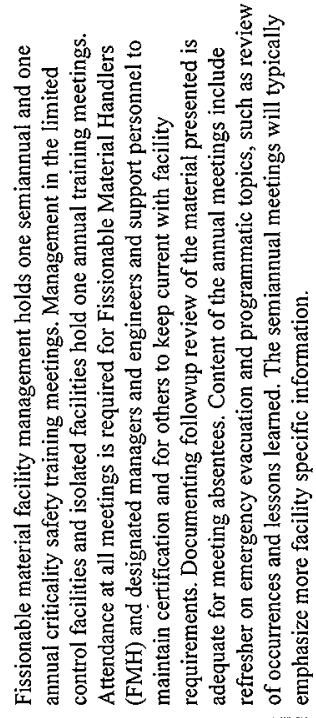 \\
\hline 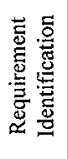 & 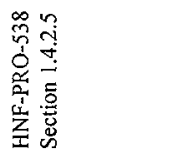 & 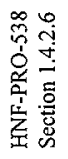 & \\
\hline 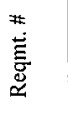 & $\begin{array}{l}\frac{m}{\infty} \\
\tilde{n}\end{array}$ & 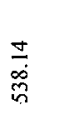 & \\
\hline
\end{tabular}

Page C-30 
TWR-3721 Rev. 0

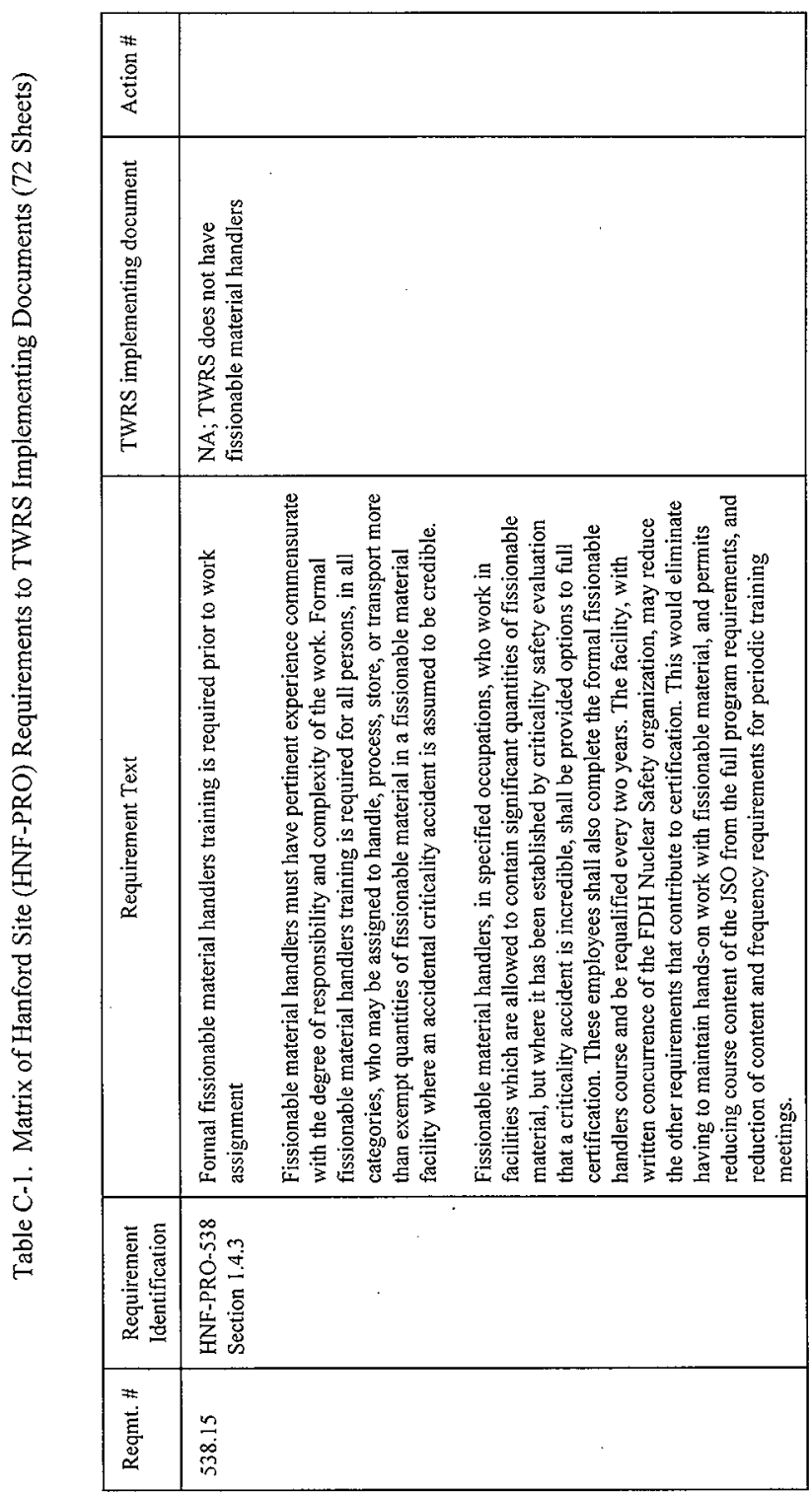

Page C-31 
TWR-3721 Rev. 0

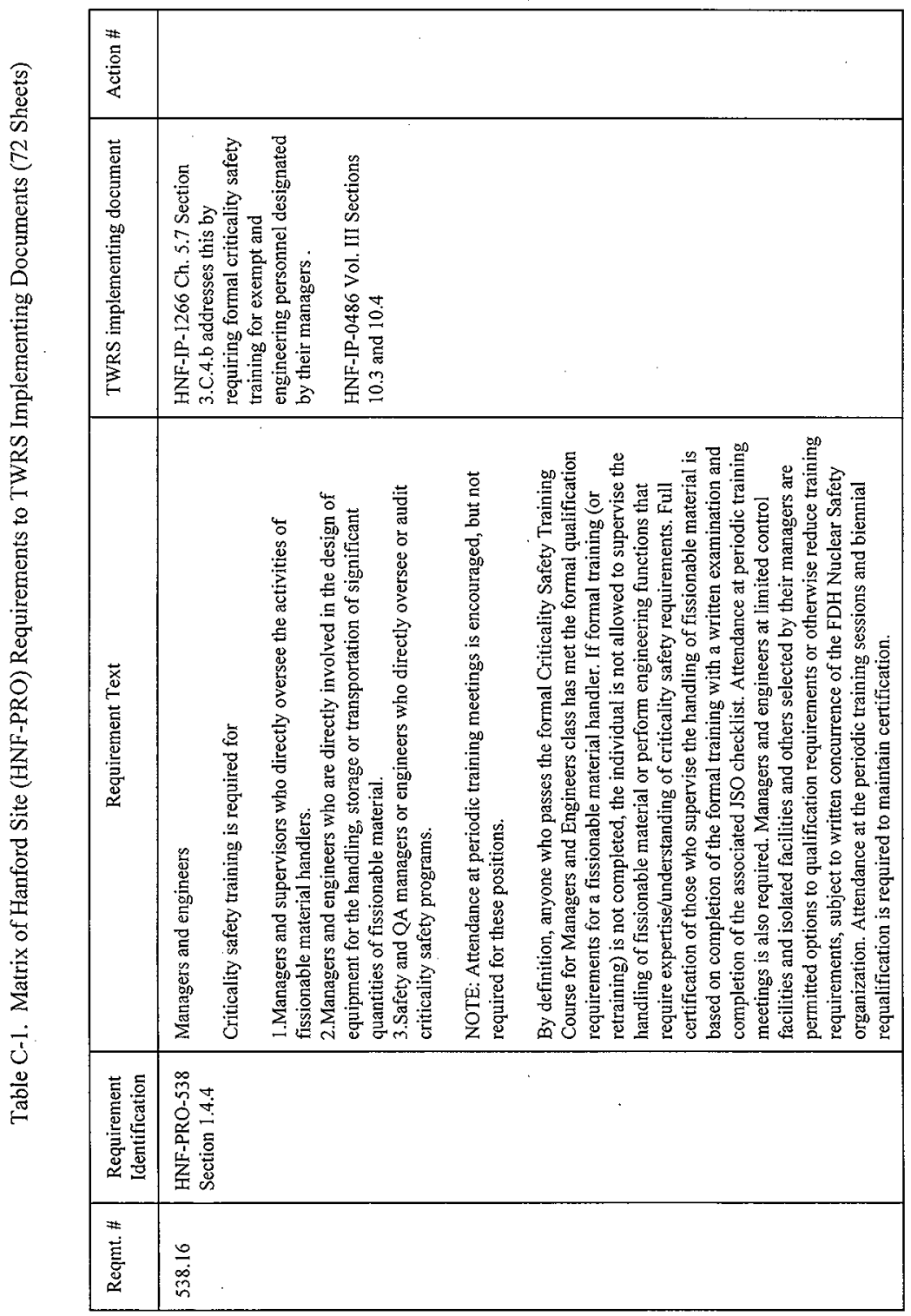

Page C-32 


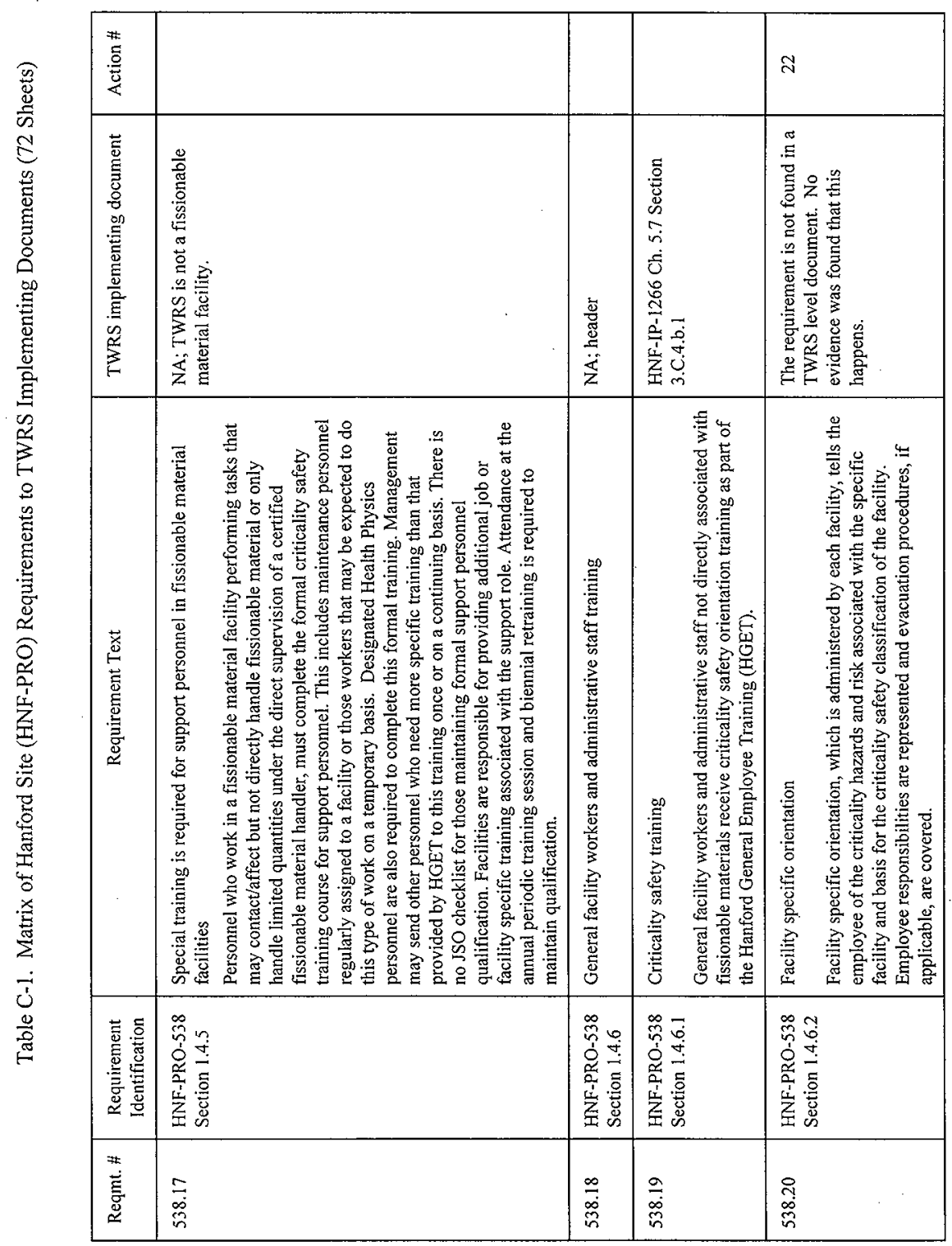

Page C-33 
TWR-3721 Rev. 0

\begin{tabular}{|c|c|c|c|c|}
\hline 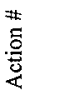 & & $\bar{N}$ & & \\
\hline 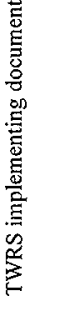 & 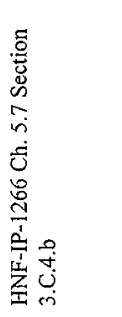 & 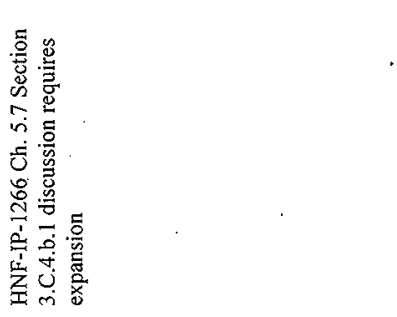 & 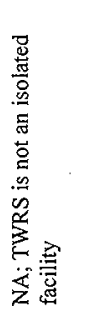 & 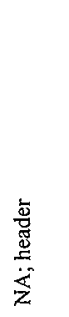 \\
\hline 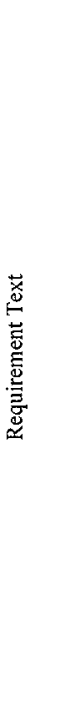 & 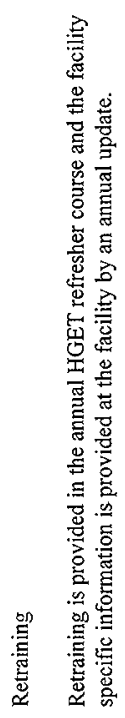 & 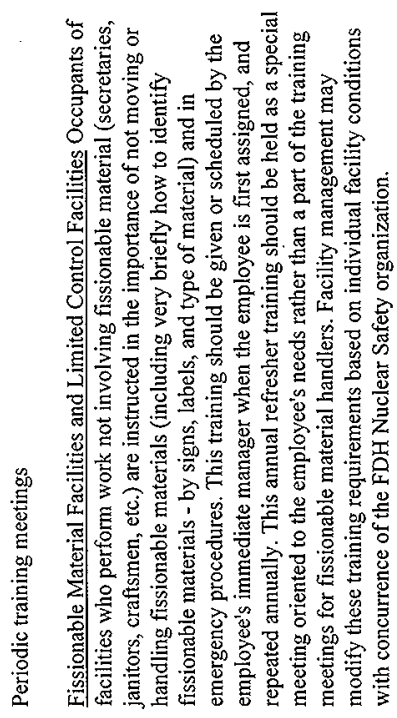 & 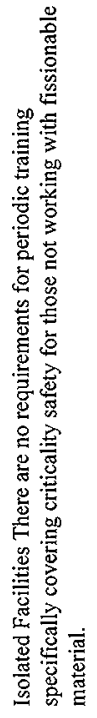 & 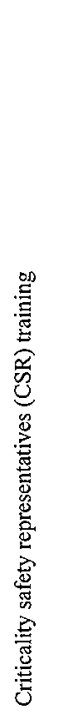 \\
\hline 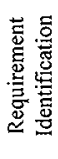 & 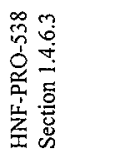 & 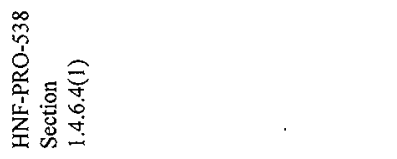 & 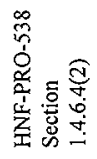 & 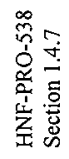 \\
\hline 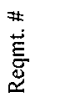 & $\begin{array}{l}\overline{\hat{n}} \\
\infty \\
i \\
i\end{array}$ & $\begin{array}{l}\tilde{N} \\
\infty \\
\infty \\
n\end{array}$ & $\begin{array}{l}\tilde{N} \\
\infty \\
\hat{n}\end{array}$ & $\begin{array}{l}\mathbb{J} \\
\infty \\
\\
\end{array}$ \\
\hline
\end{tabular}


TWR-3721 Rev. 0

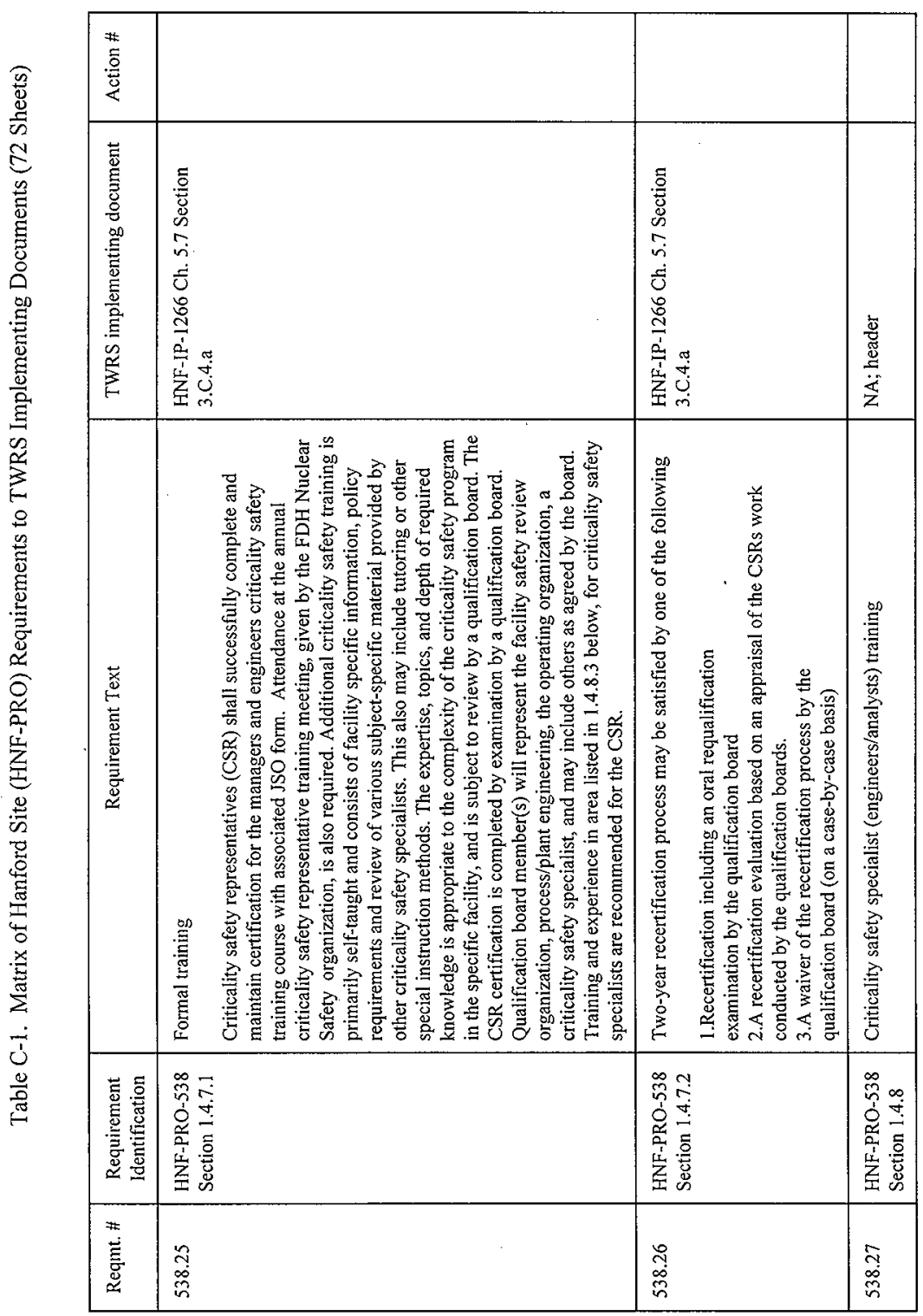

Page C-35 
TWR-3721 Rev. 0

\begin{tabular}{|c|c|c|c|c|c|c|}
\hline 雚 & 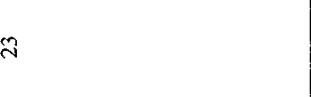 & $\tilde{\Upsilon}$ & \multicolumn{2}{|l|}{$\ddot{\mathrm{i}}$} & \multicolumn{2}{|l|}{ 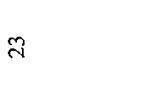 } \\
\hline 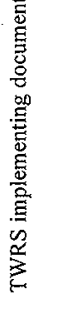 & 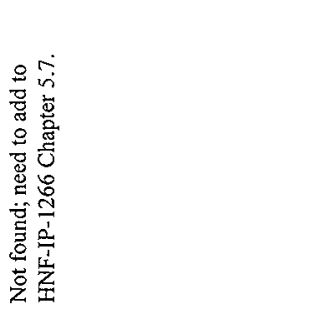 & 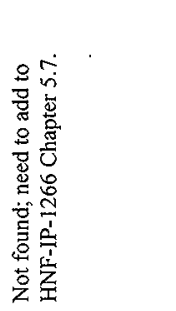 & 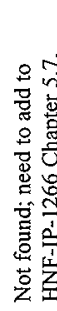 & . & 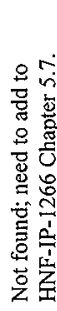 & \\
\hline 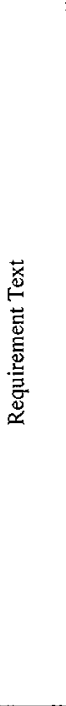 & 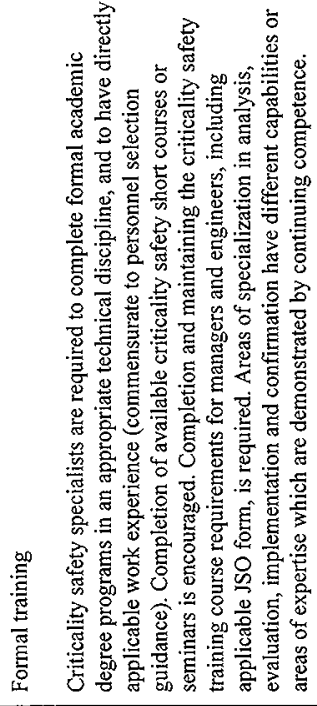 & 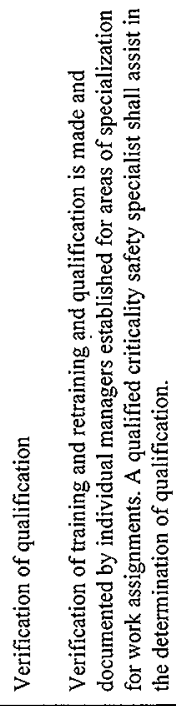 & 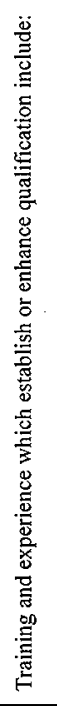 & 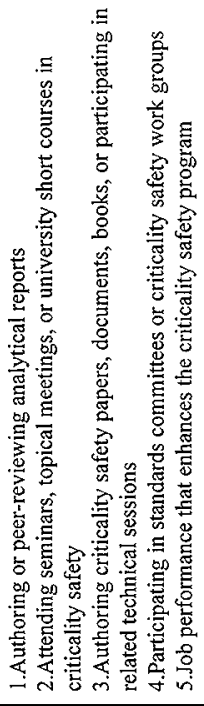 & 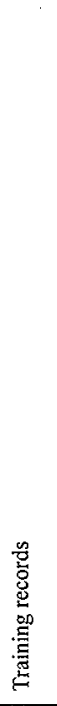 & 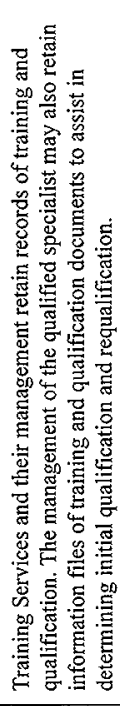 \\
\hline 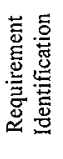 & 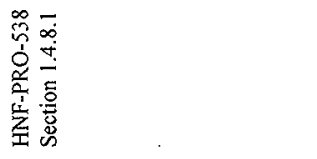 & 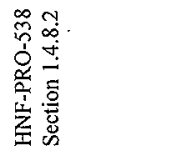 & 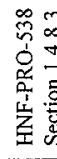 & & 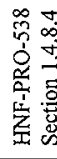 & \\
\hline 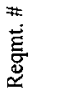 & $\begin{array}{l}\text { 心్ } \\
\infty \\
\infty \\
n\end{array}$ & 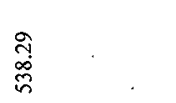 & $\begin{array}{l}0 \\
\infty \\
\infty \\
\infty \\
i n\end{array}$ & & $\begin{array}{l}\bar{m} \\
\infty \\
0 \\
i n\end{array}$ & \\
\hline
\end{tabular}


TWR-3721 Rev. 0

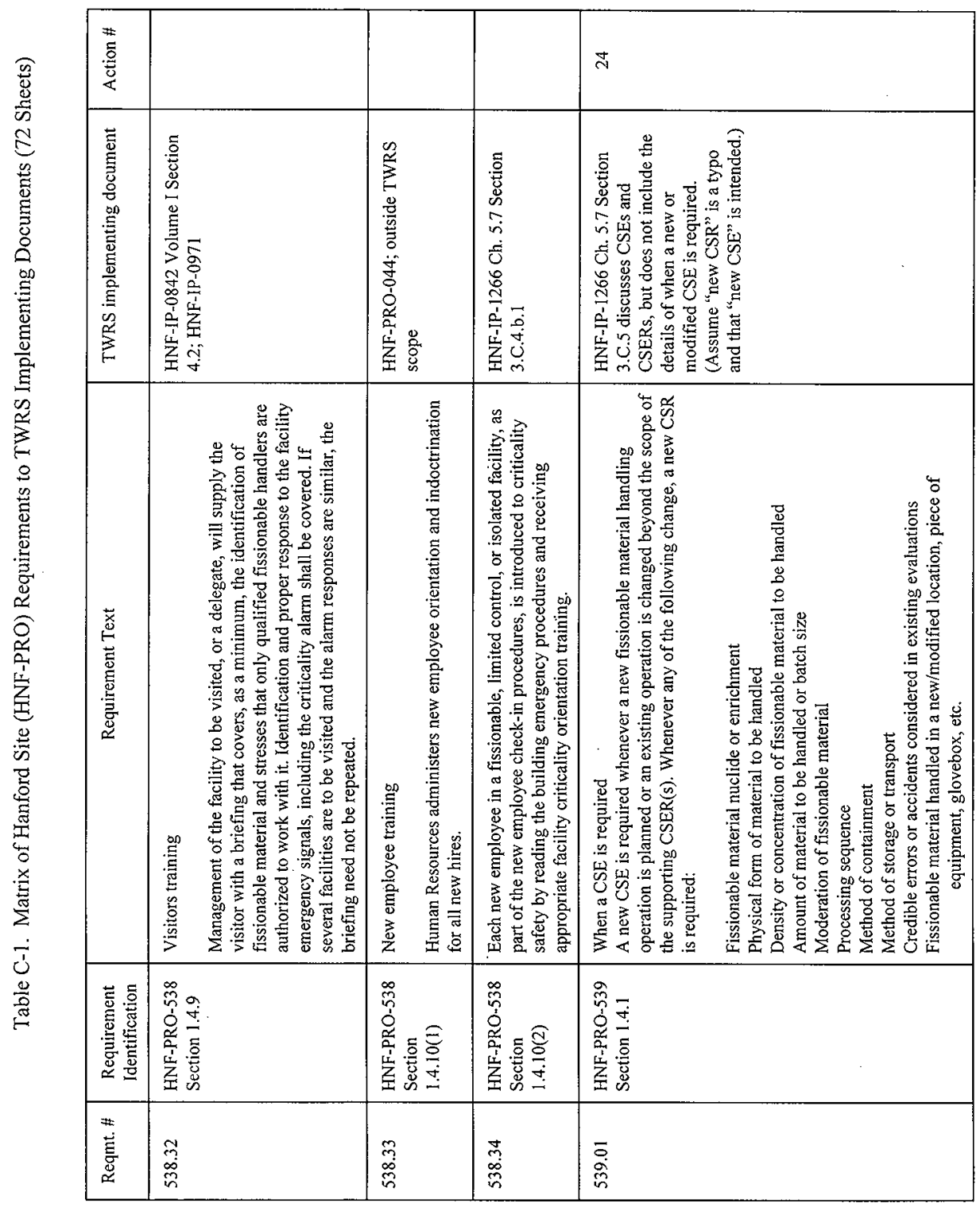

Page C-37 
TWR-3721 Rev. 0

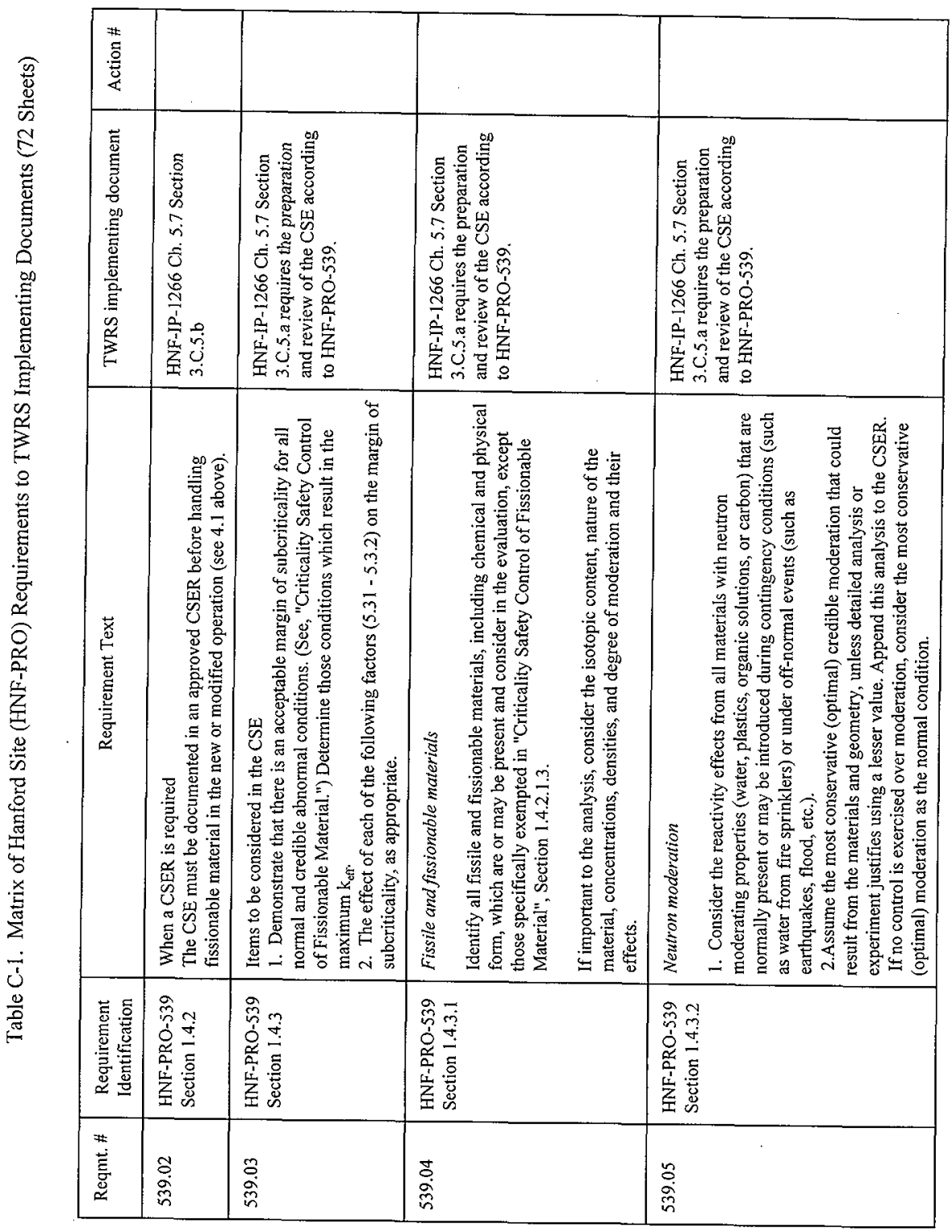


TWR-3721 Rev. 0

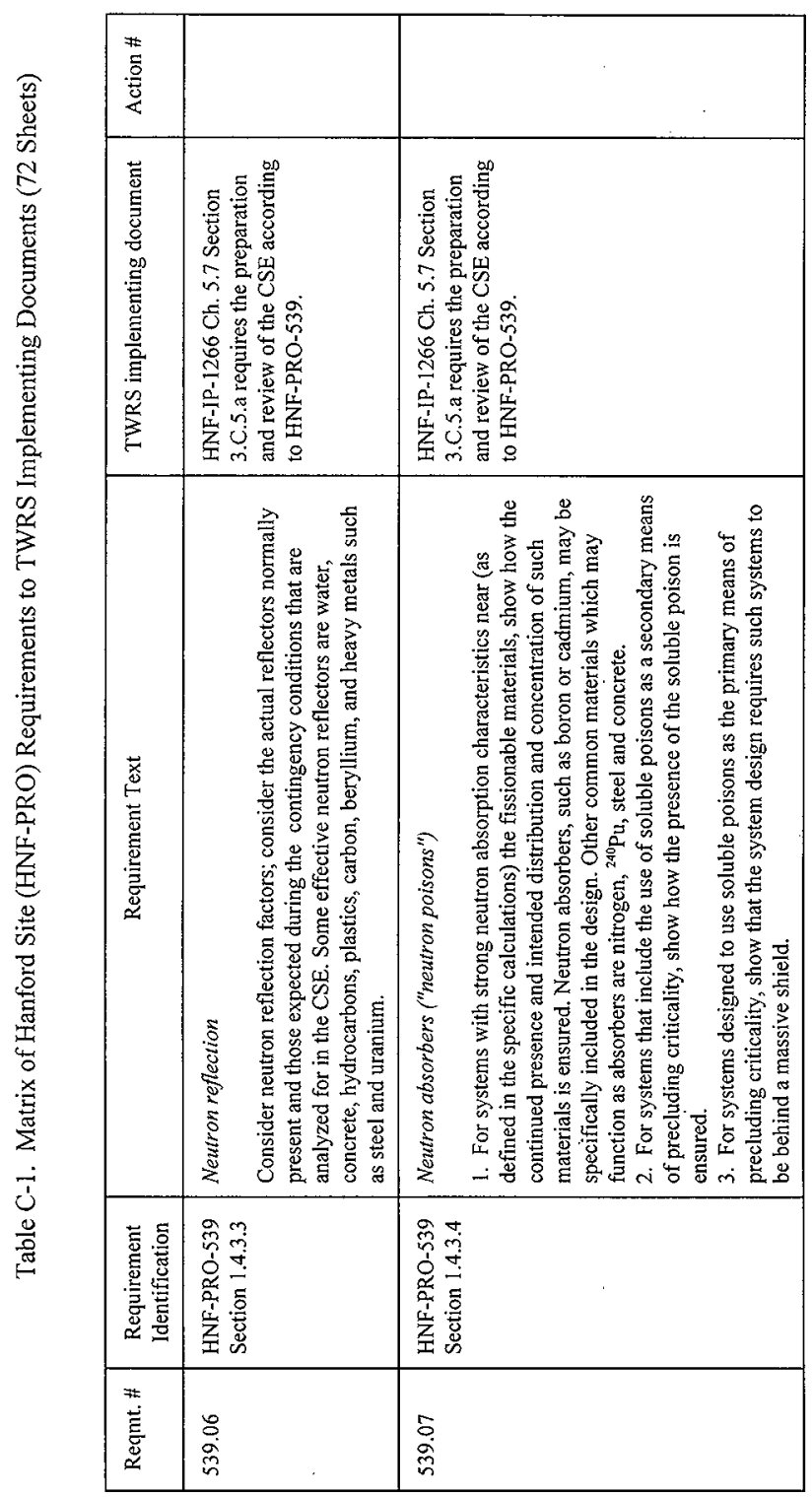

Page C-39 
TWR-3721 Rev. 0

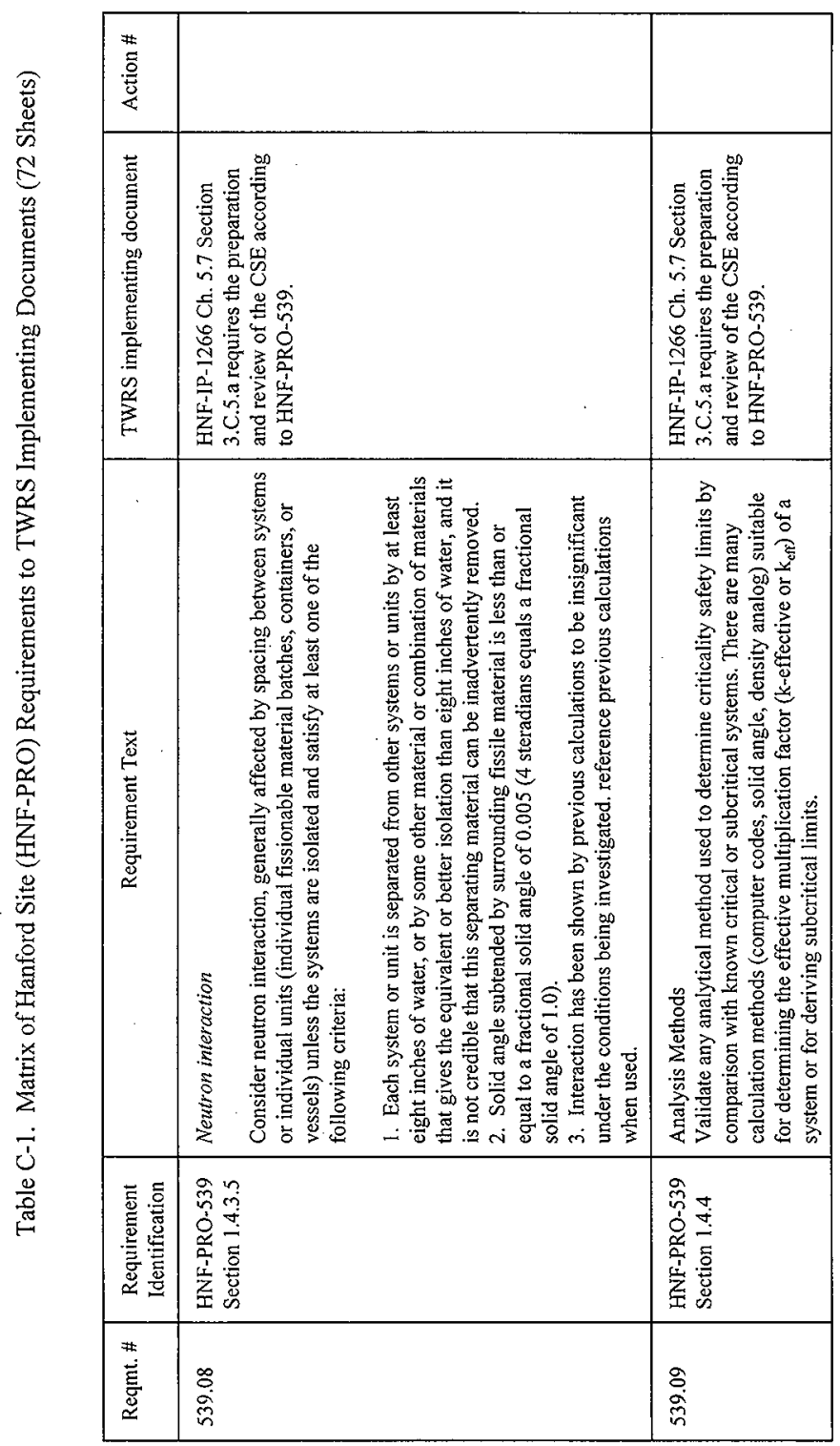

Page C-40 
TWR-3721 Rev. 0

\begin{tabular}{|c|c|c|c|}
\hline 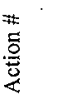 & & & \\
\hline 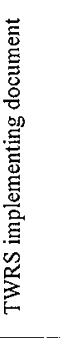 & 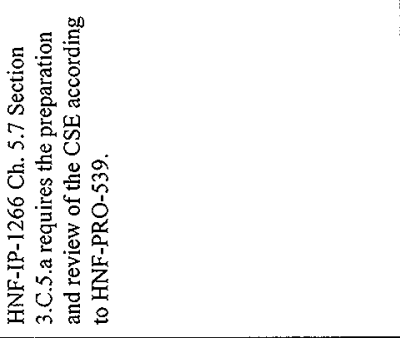 & 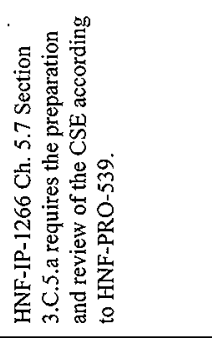 & \\
\hline 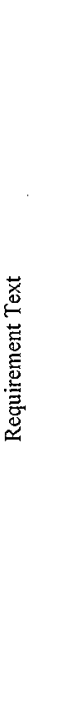 & 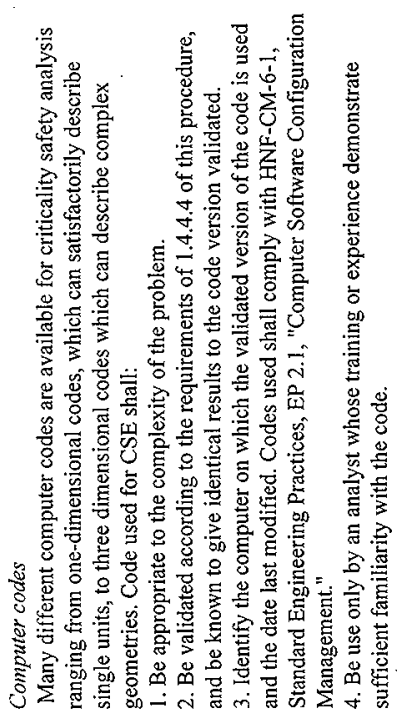 & 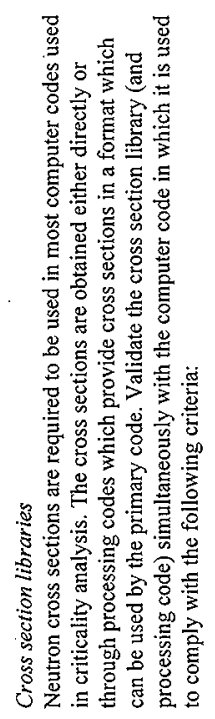 & 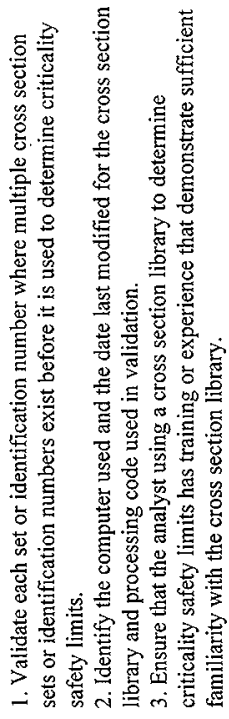 \\
\hline 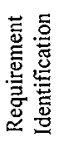 & 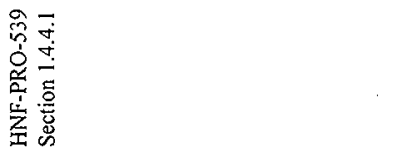 & 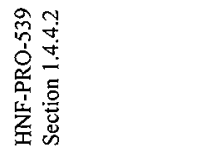 & \\
\hline 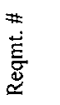 & $\frac{}{\stackrel{\circ}{\sigma}}$ & $=$ & \\
\hline
\end{tabular}


TWR-3721 Rev. 0

\begin{tabular}{|c|c|c|c|c|}
\hline 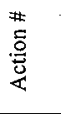 & & & & \\
\hline 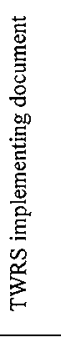 & 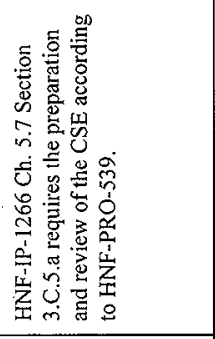 & 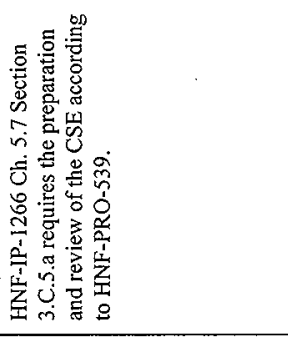 & 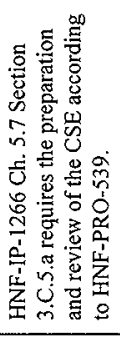 & 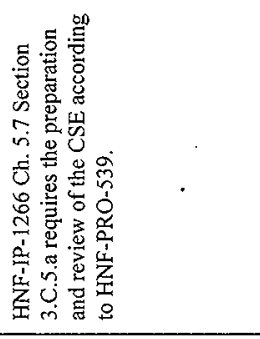 \\
\hline 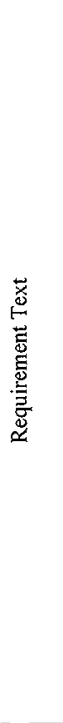 & 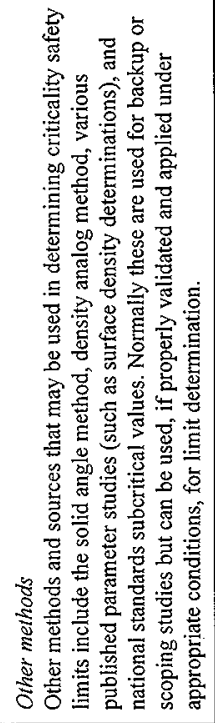 & 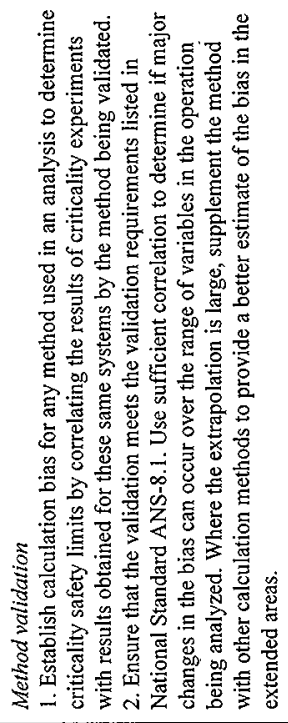 & 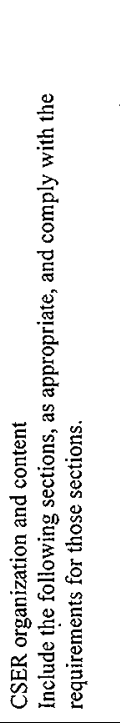 & 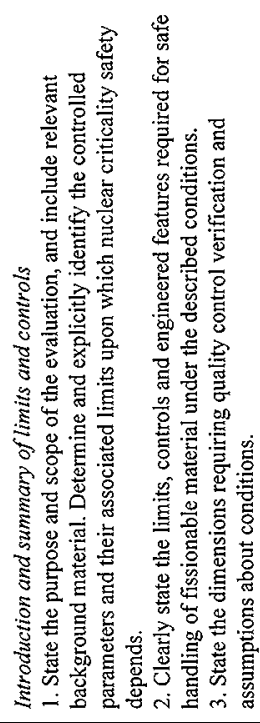 \\
\hline 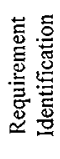 & 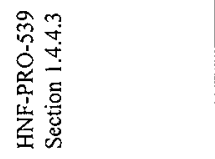 & 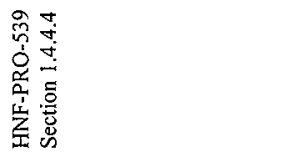 & 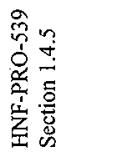 & 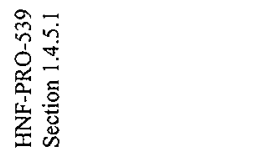 \\
\hline 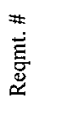 & 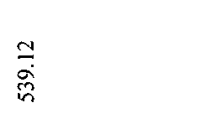 & $\stackrel{m}{\circ}$ & $\frac{\square}{a}$ & $\frac{n}{a}$ \\
\hline
\end{tabular}


TWR-3721 Rev. 0

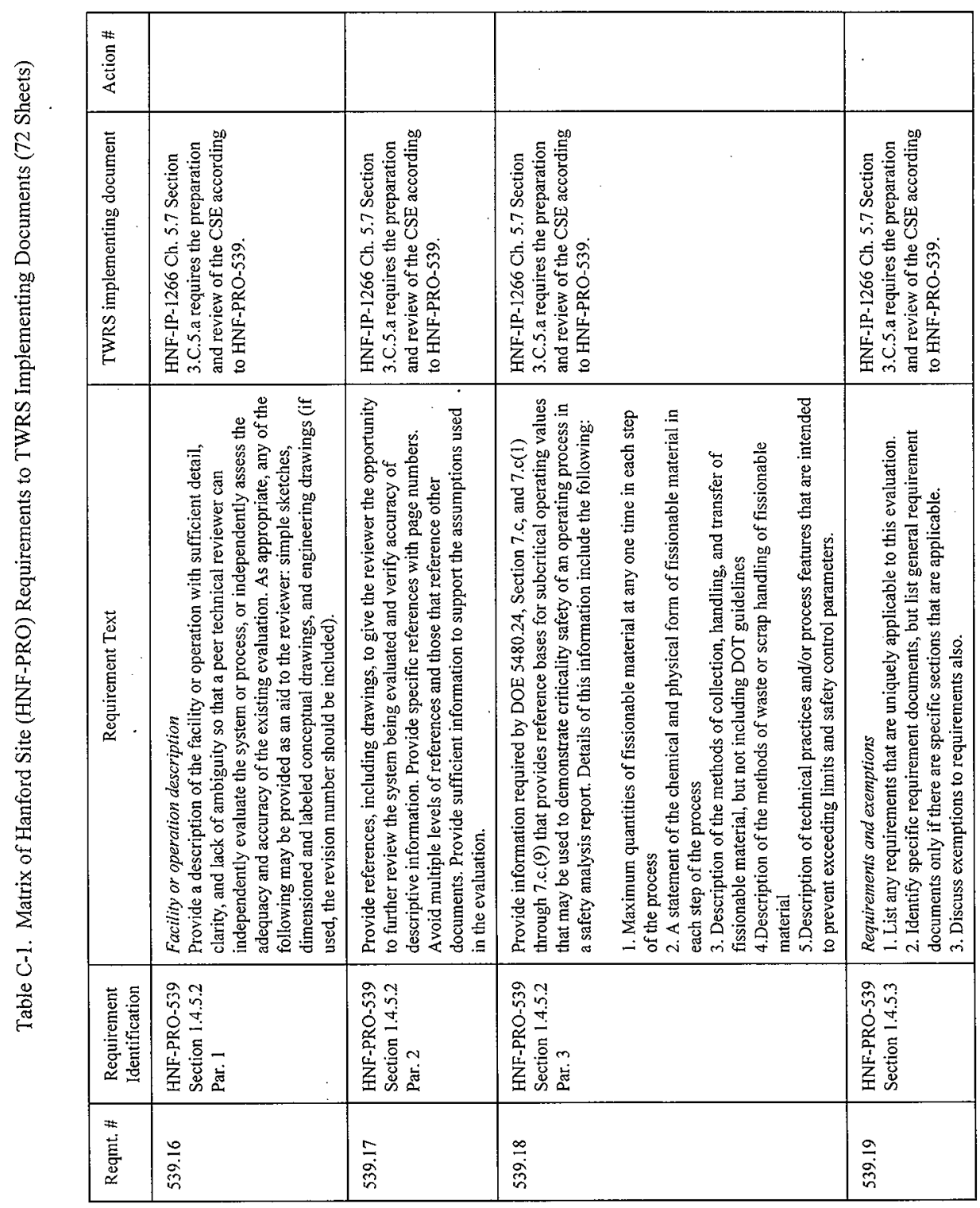


TWR-3721 Rev. 0

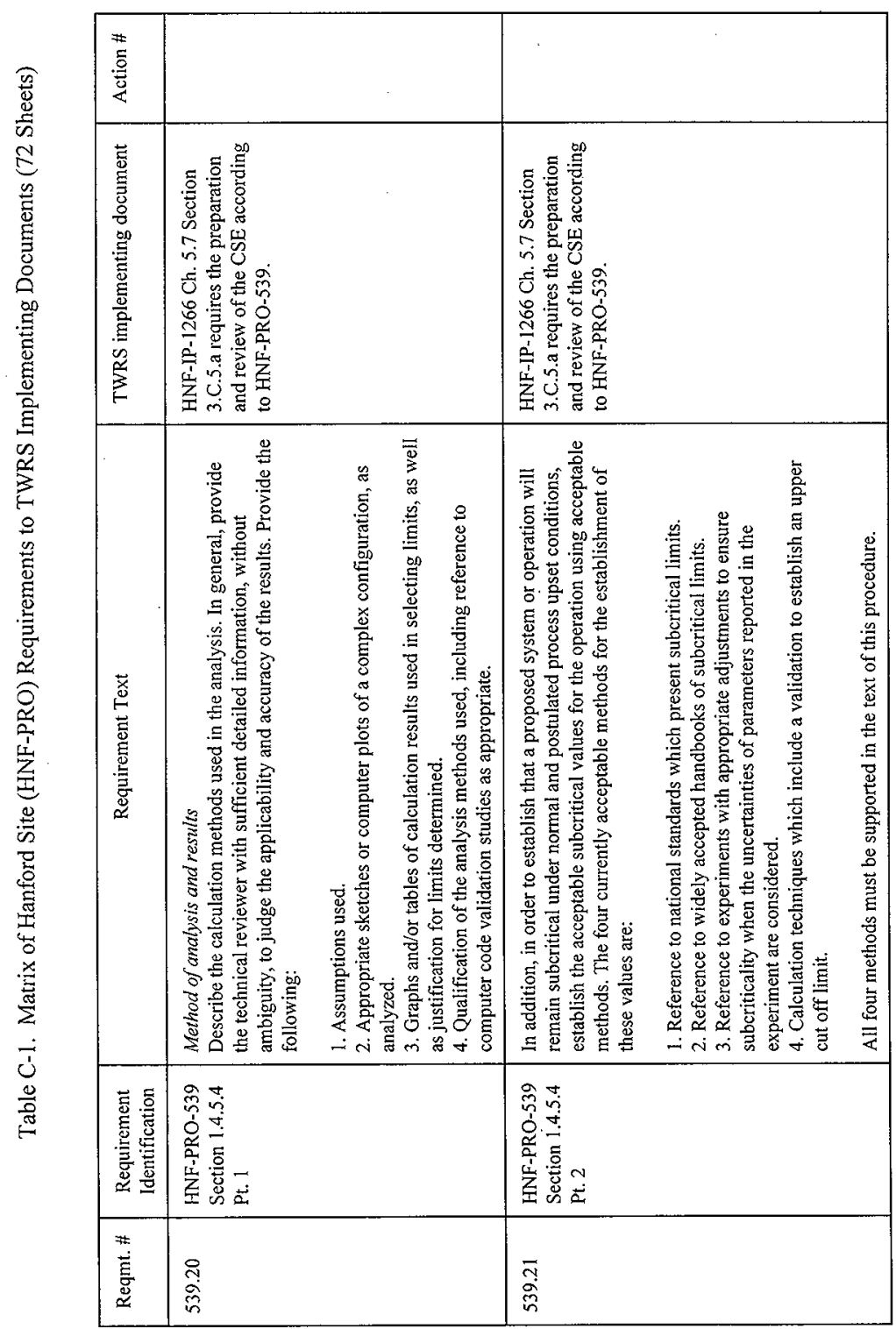

Page C-44 
TWR-3721 Rev. 0

\begin{tabular}{|c|c|c|c|c|}
\hline 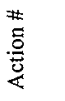 & & & & \\
\hline 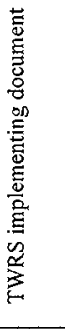 & 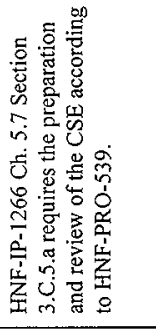 & & 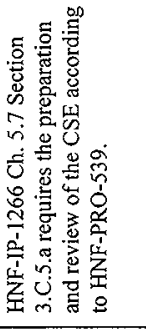 & 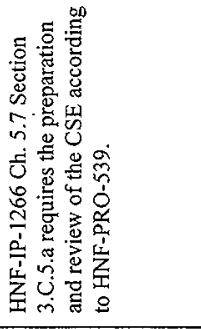 \\
\hline 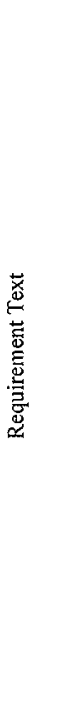 & 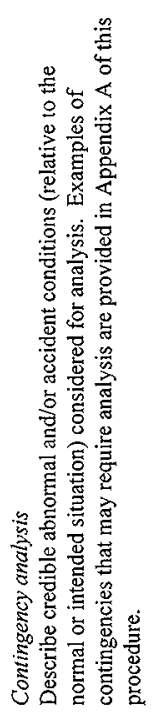 & 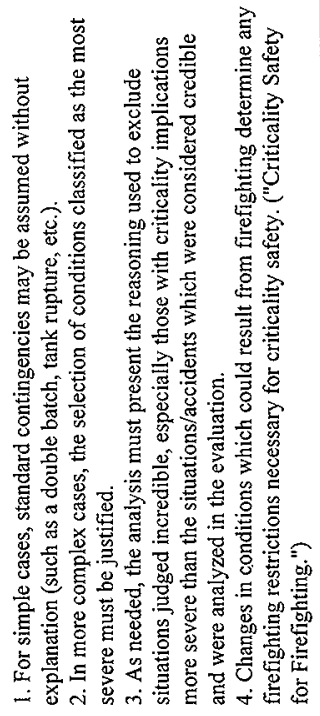 & 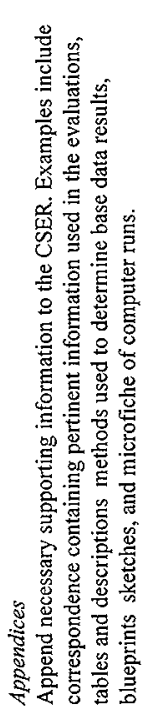 & 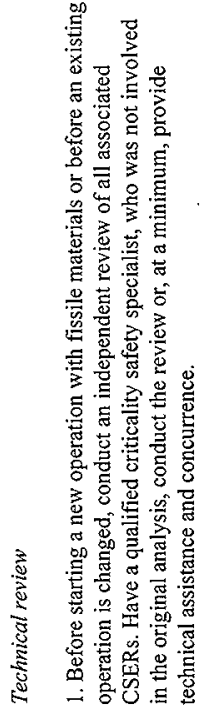 \\
\hline 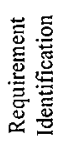 & 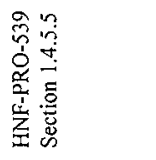 & & 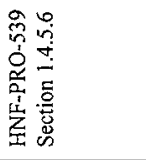 & 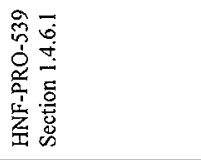 \\
\hline $\begin{array}{l}\text { \# } \\
\text { 蓠 } \\
\stackrel{\Xi}{\cong}\end{array}$ & 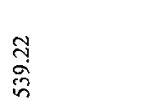 & & $\begin{array}{l}\hat{n} \\
\stackrel{s}{s}\end{array}$ & $\begin{array}{l}\text { స్ } \\
\text { ğ } \\
\text { ñ }\end{array}$ \\
\hline
\end{tabular}


TWR-3721 Rev. 0

\begin{tabular}{|c|c|c|c|c|c|c|c|c|}
\hline $\begin{array}{l}\text { \# } \\
\frac{5}{0} \\
\frac{\pi}{2}\end{array}$ & & & & & & & & \\
\hline 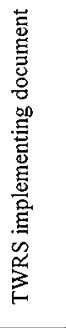 & \multicolumn{2}{|c|}{ 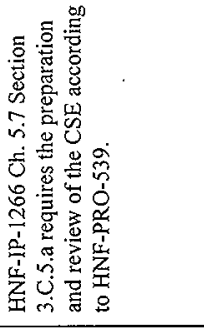 } & 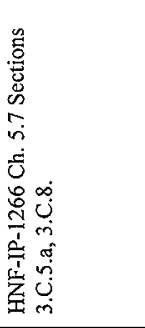 & 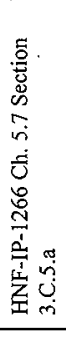 & 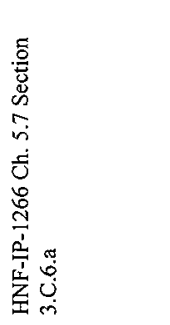 & \multicolumn{2}{|c|}{ 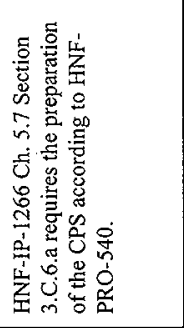 } & 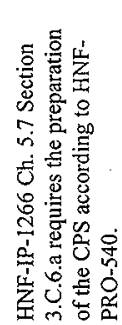 \\
\hline 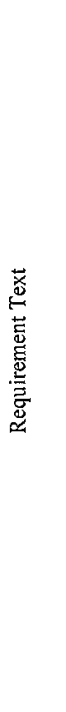 & . & 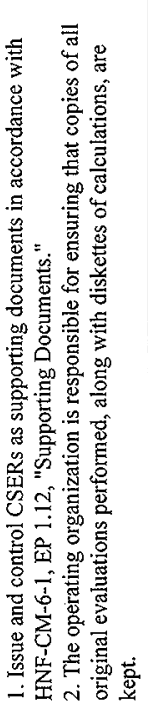 & 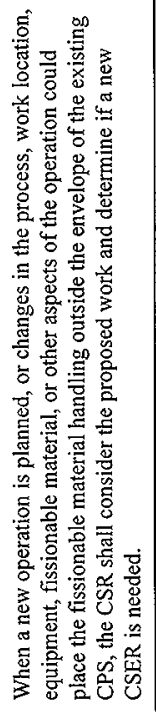 & 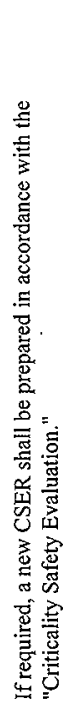 & 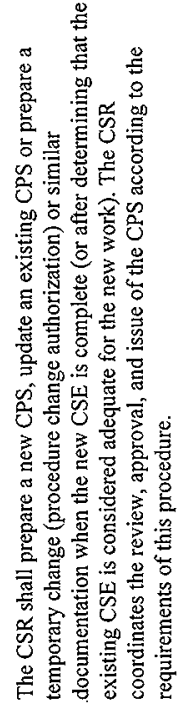 & 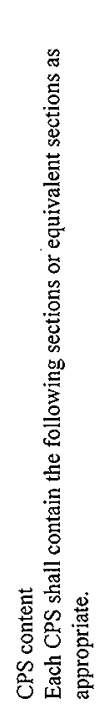 & 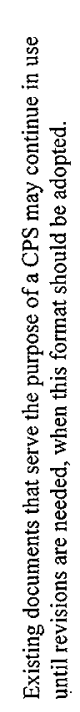 & 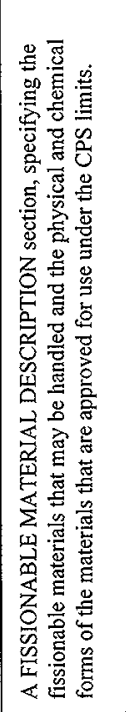 \\
\hline 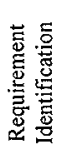 & 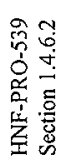 & & 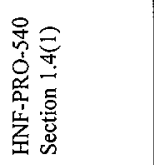 & 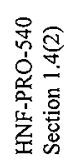 & 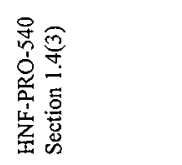 & 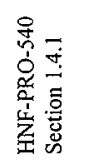 & & 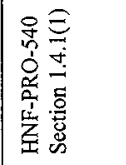 \\
\hline 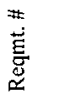 & 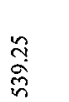 & & $\begin{array}{l}\vec{\circ} \\
\text { : }\end{array}$ & 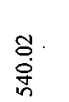 & $\begin{array}{l}\stackrel{3}{0} \\
\stackrel{9}{0}\end{array}$ & $\begin{array}{l}\text { D } \\
\stackrel{0}{0}\end{array}$ & & $\begin{array}{l}\text { o. } \\
\text { if } \\
\text { in }\end{array}$ \\
\hline
\end{tabular}


TWR-3721 Rev. 0

\begin{tabular}{|c|c|c|c|c|c|c|}
\hline$\frac{\tilde{g}}{\stackrel{\Xi}{8}}$ & & & & & & \\
\hline 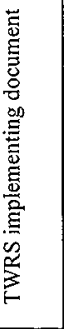 & 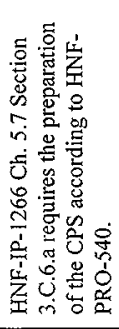 & 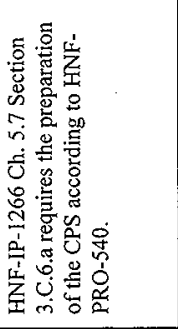 & 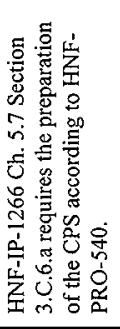 & 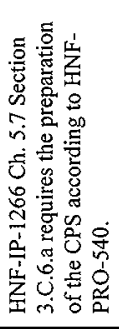 & 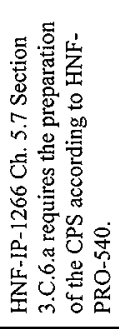 & 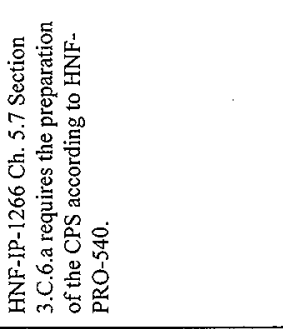 \\
\hline 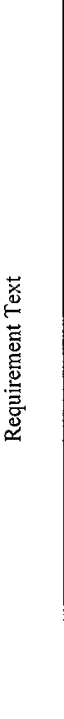 & 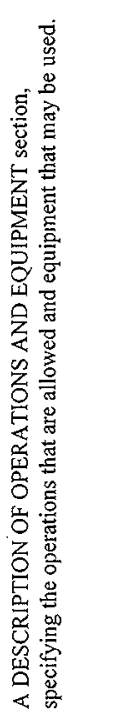 & 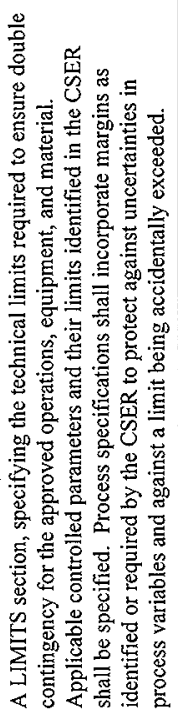 & 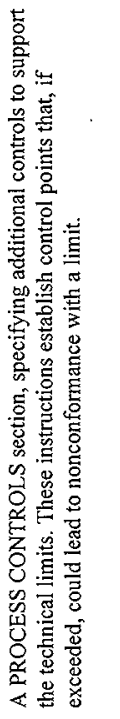 & 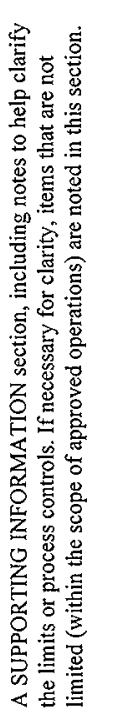 & 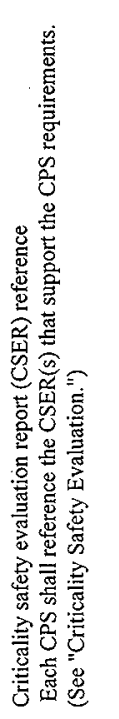 & 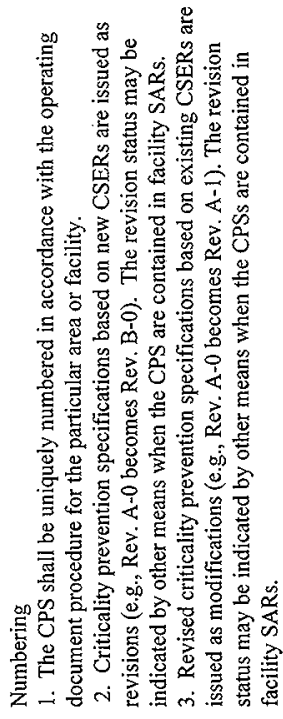 \\
\hline 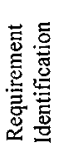 & 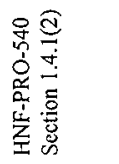 & 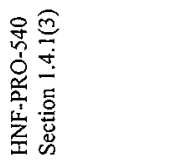 & 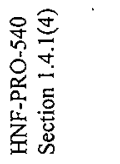 & 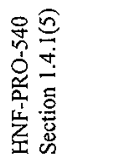 & 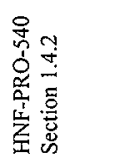 & 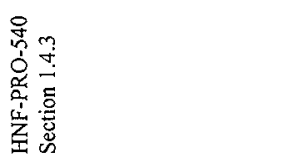 \\
\hline 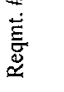 & $\begin{array}{l}\stackrel{8}{\circ} \\
\stackrel{8}{8} \\
\text { in }\end{array}$ & $\begin{array}{l}5 \\
\stackrel{0}{0} \\
\text { 요 }\end{array}$ & $\begin{array}{l}\stackrel{\infty}{\circ} \\
\stackrel{8}{\oplus} \\
\stackrel{H}{\circ}\end{array}$ & $\begin{array}{l}\stackrel{g}{0} \\
\stackrel{8}{0}\end{array}$ & $\begin{array}{l}\stackrel{9}{\circ} \\
\text { 宫 }\end{array}$ & $\begin{array}{l}\overline{0} \\
\text { 莴 }\end{array}$ \\
\hline
\end{tabular}


TWR-3721 Rev. 0

\begin{tabular}{|c|c|c|c|c|c|c|}
\hline $\begin{array}{l}\text { : } \\
\text { 咅 } \\
\stackrel{0}{<}\end{array}$ & & & & & & \\
\hline 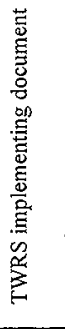 & 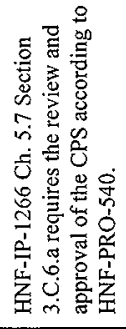 & 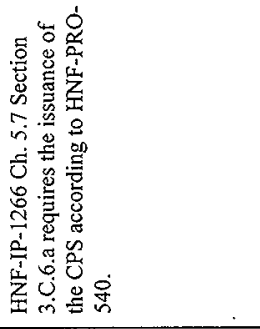 & 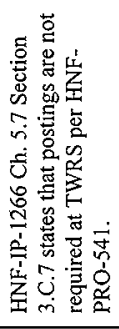 & 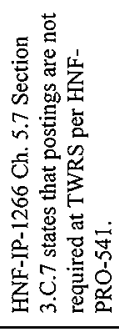 & 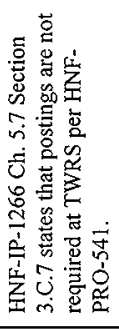 & 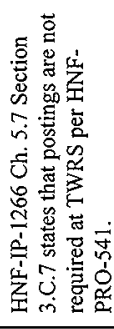 \\
\hline 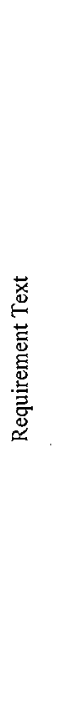 & 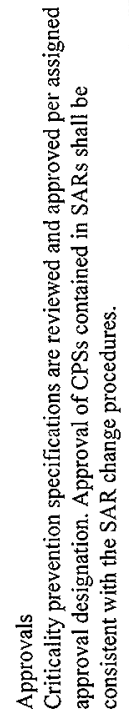 & 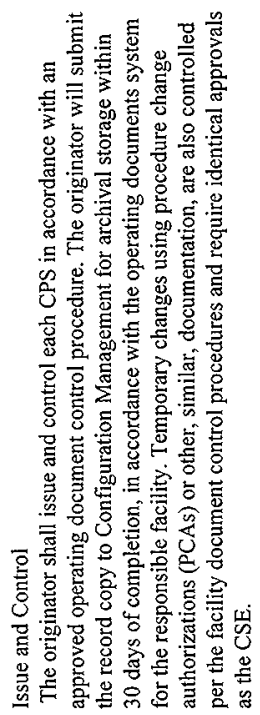 & 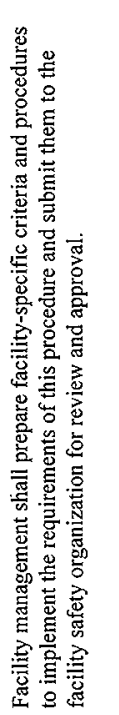 & 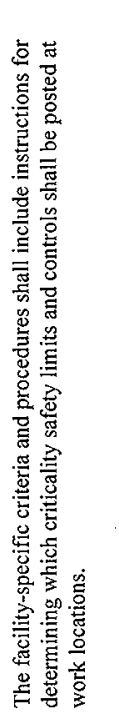 & 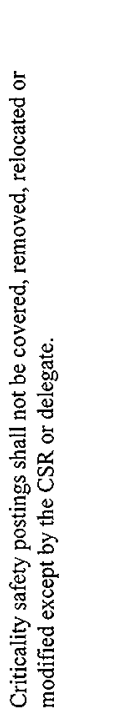 & 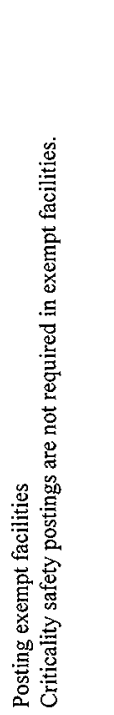 \\
\hline 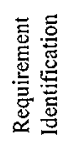 & 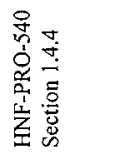 & 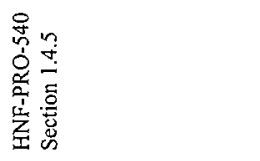 & 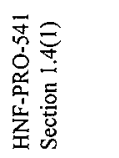 & 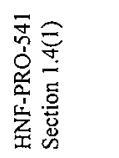 & 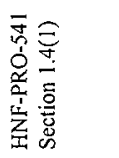 & 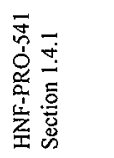 \\
\hline 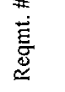 & $\begin{array}{l}\text { T } \\
\text { 옴 }\end{array}$ & $\stackrel{m}{\stackrel{m}{0}}$ & $\frac{\bar{\Xi}}{\vec{\nabla}}$ & $\frac{\mathscr{d}}{\bar{\nabla}}$ & 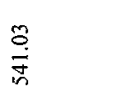 & 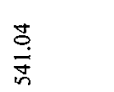 \\
\hline
\end{tabular}


TWR-3721 Rev. 0

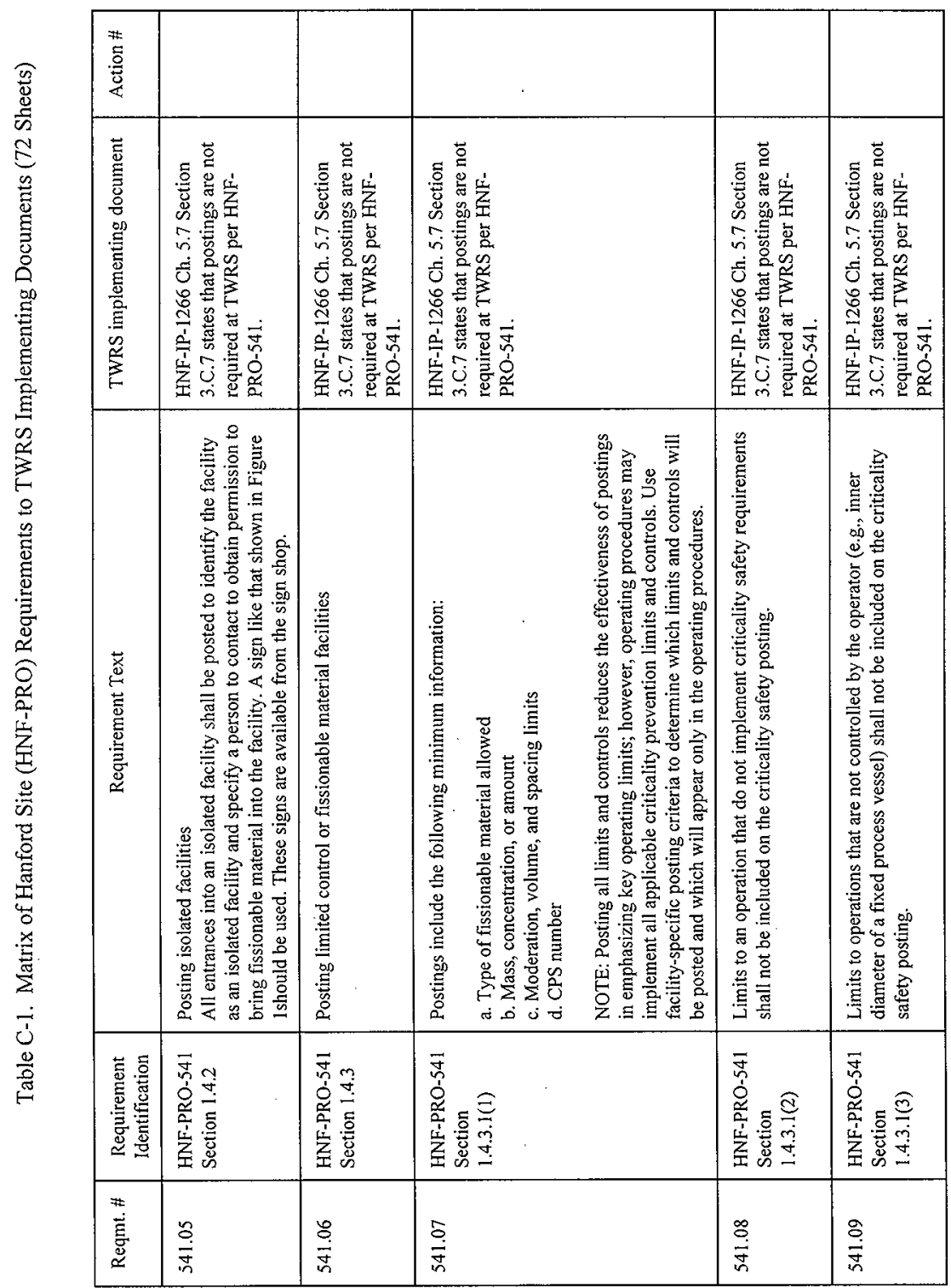


TWR-3721 Rev. 0

\begin{tabular}{|c|c|c|c|c|c|c|c|}
\hline 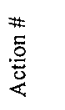 & & & & & & & \\
\hline 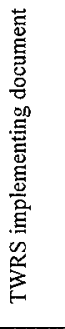 & 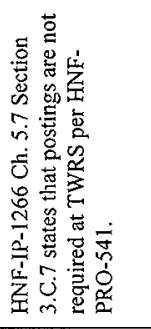 & 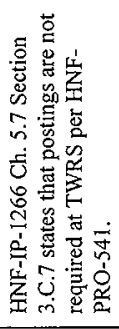 & 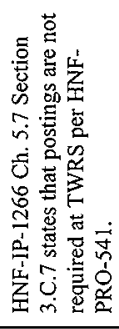 & 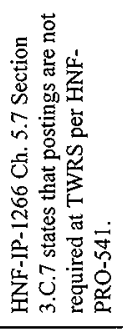 & 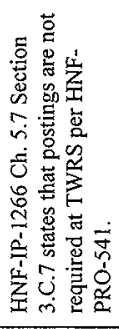 & 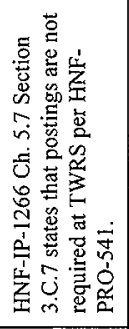 & 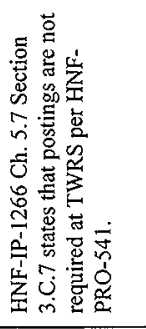 \\
\hline 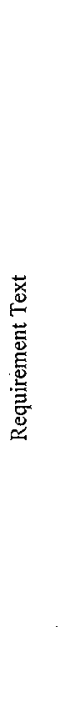 & 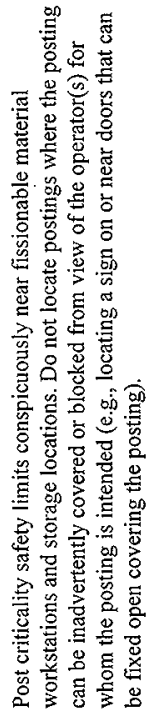 & 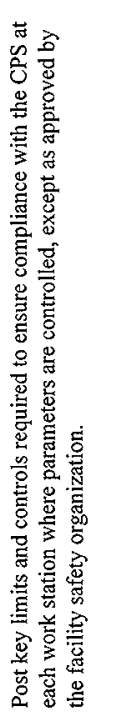 & 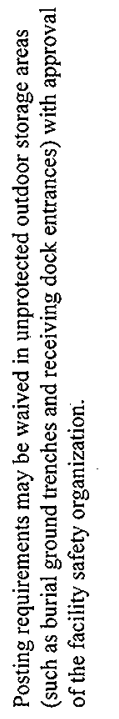 & 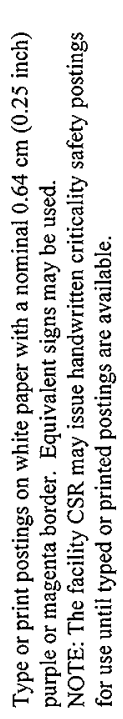 & 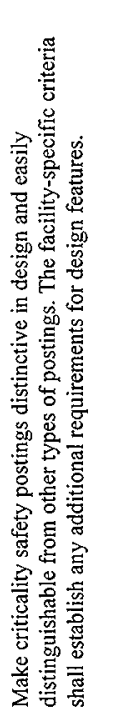 & 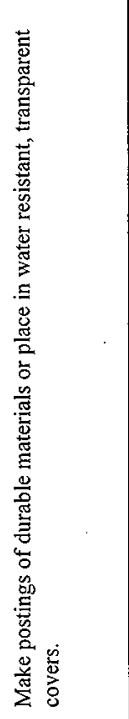 & 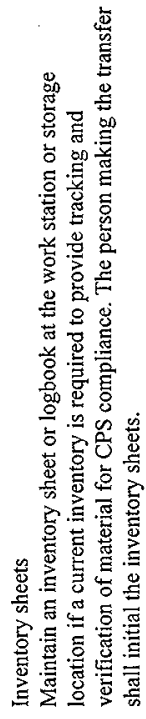 \\
\hline 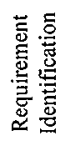 & 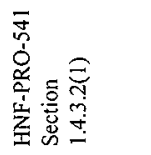 & 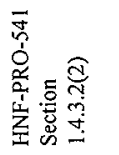 & 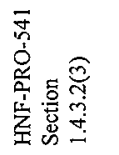 & 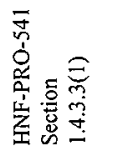 & 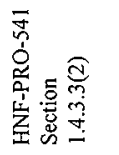 & 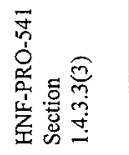 & 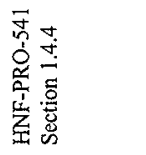 \\
\hline 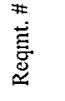 & 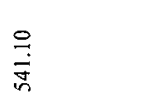 & 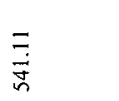 & 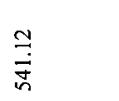 & $\frac{9}{\longrightarrow}$ & $\frac{ \pm}{\dot{\mathbb{G}}}$ & $\frac{n}{\dot{m}}$ & $\begin{array}{l}\frac{0}{4} \\
\text { 苛 }\end{array}$ \\
\hline
\end{tabular}


TWR-3721 Rev. 0

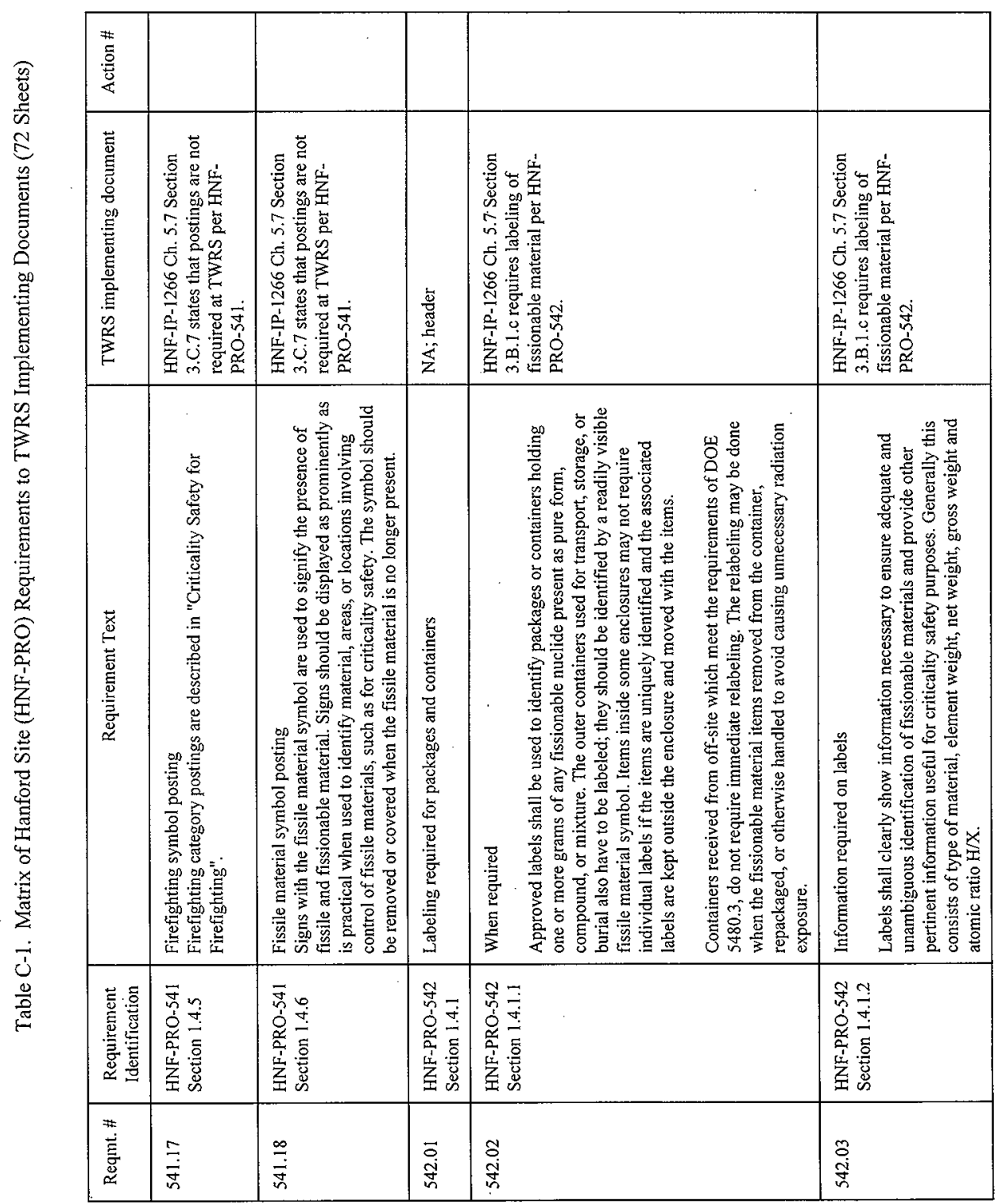


TWR-3721 Rev. 0

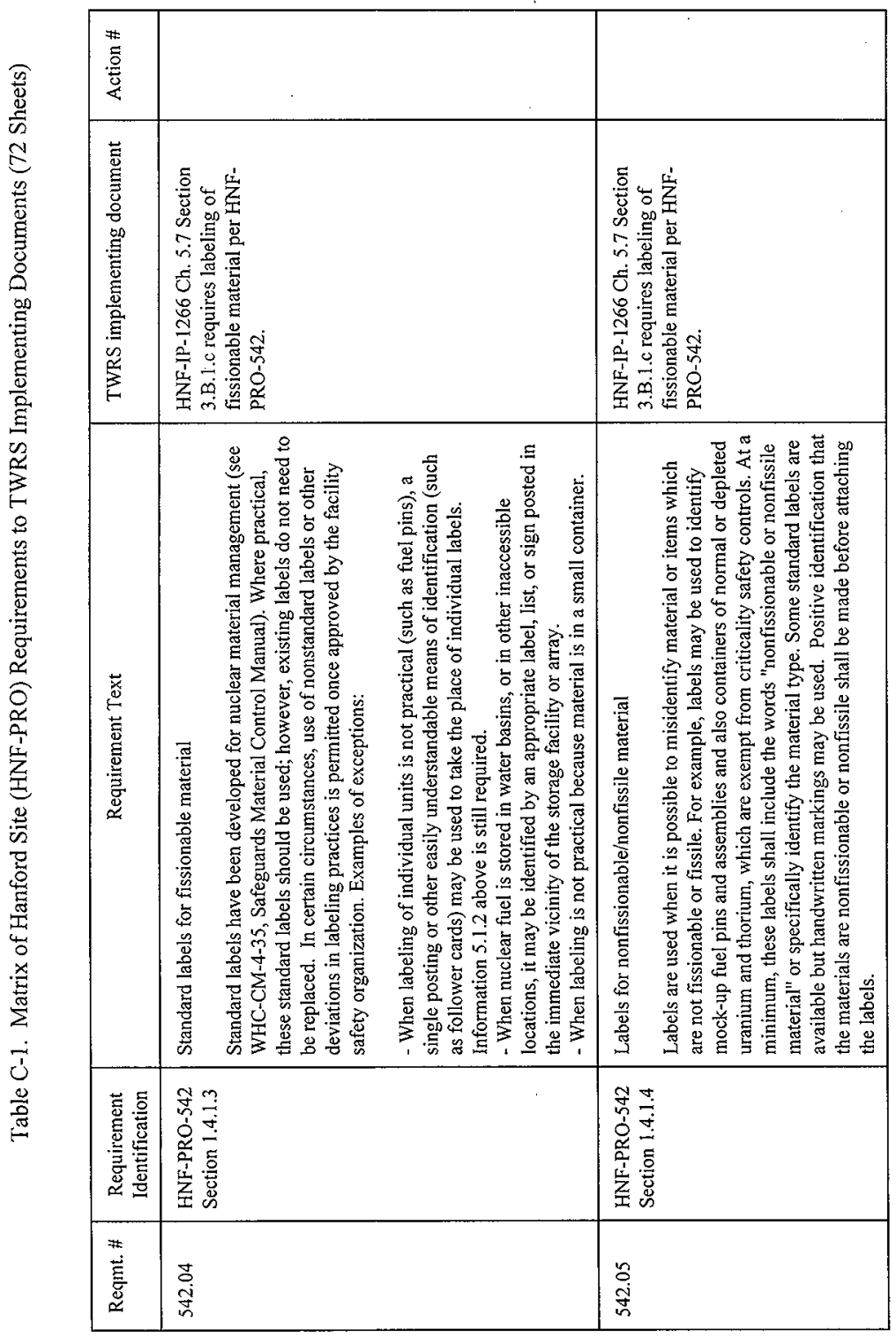

Page C-52 
TWR-3721 Rev. 0

\begin{tabular}{|c|c|c|c|c|c|}
\hline 范 & & & & & \\
\hline 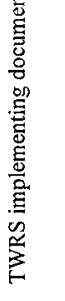 & 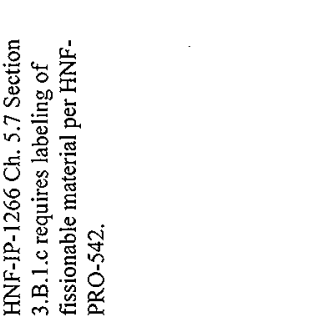 & 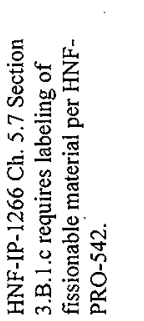 & 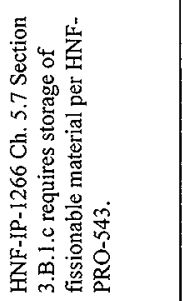 & 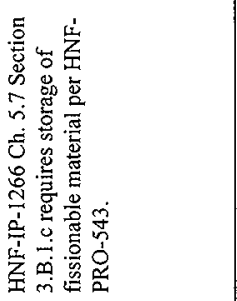 & 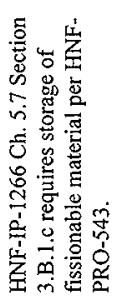 \\
\hline 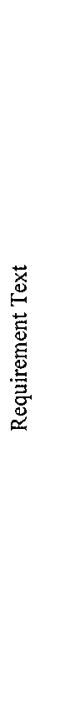 & 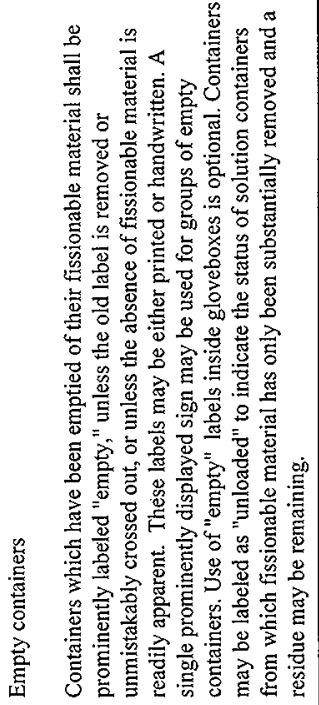 & 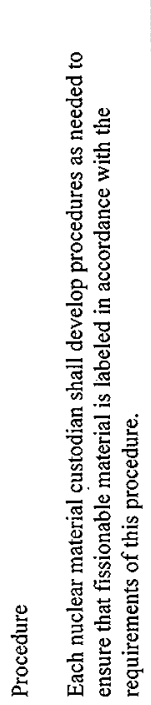 & 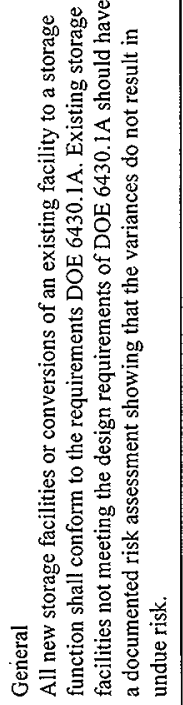 & 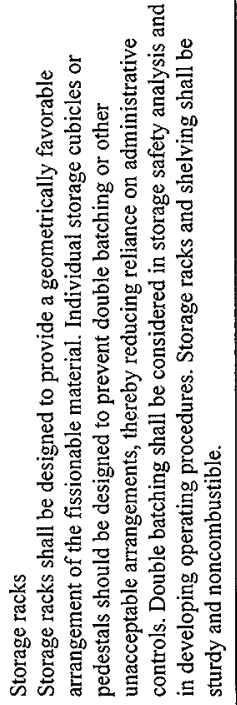 & 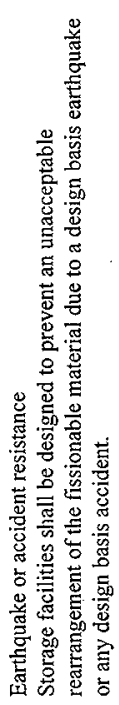 \\
\hline 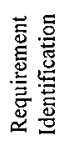 & 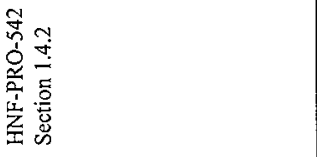 & 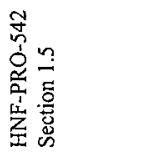 & 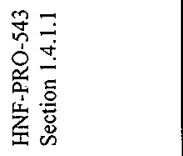 & 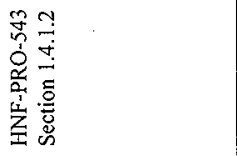 & 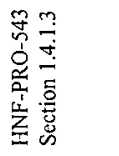 \\
\hline 苐 & $\begin{array}{l}\stackrel{8}{0} \\
\text { i } \\
\text { Hi }\end{array}$ & $\begin{array}{l}5 \\
\stackrel{\circ}{N} \\
\text { ज. }\end{array}$ & $\begin{array}{l}\overrightarrow{0} \\
\stackrel{\sim}{n}\end{array}$ & $\begin{array}{l}\mathcal{Y} \\
\stackrel{9}{0} \\
\mathcal{W}\end{array}$ & 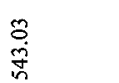 \\
\hline
\end{tabular}


TWR-3721 Rev. 0

\begin{tabular}{|c|c|c|c|c|c|c|}
\hline 洁 & & & . & & & \\
\hline 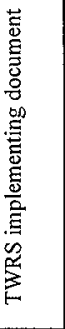 & 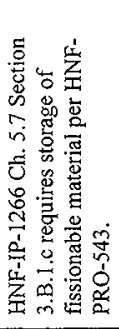 & 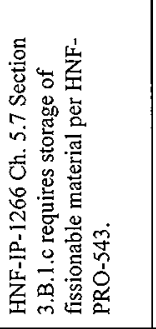 & 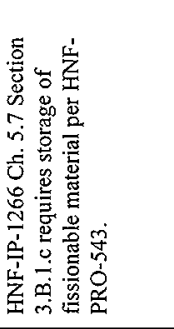 & 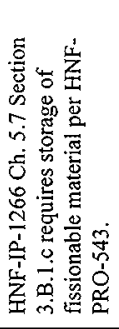 & 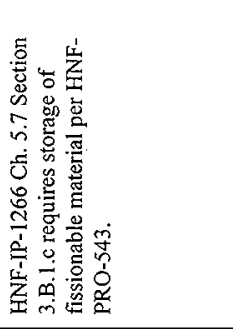 & 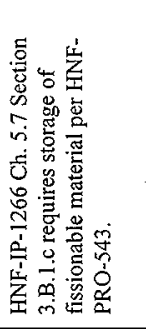 \\
\hline 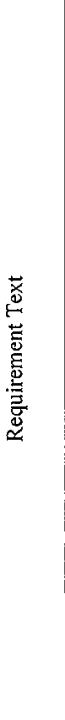 & 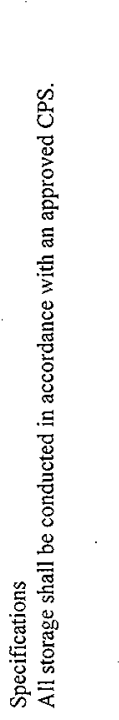 & 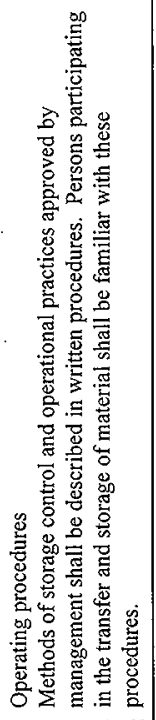 & 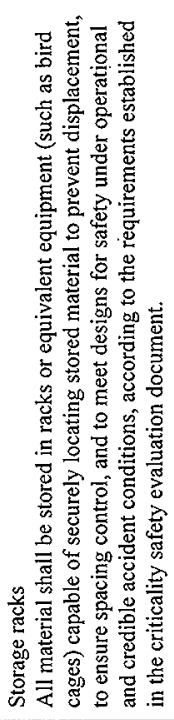 & 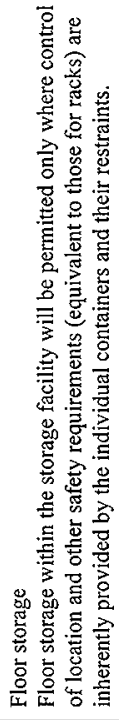 & 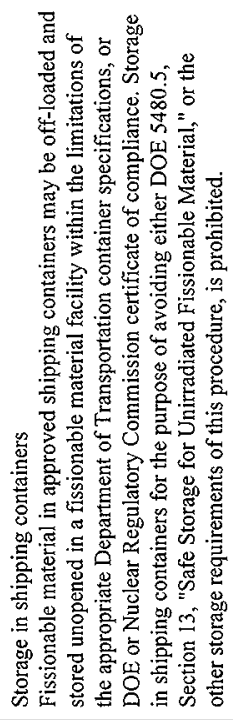 & 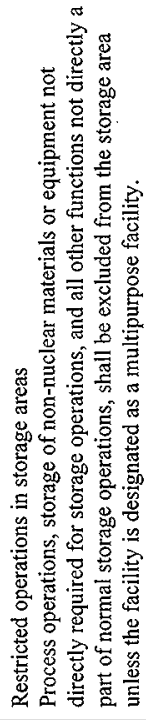 \\
\hline 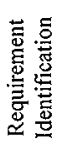 & 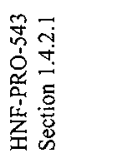 & 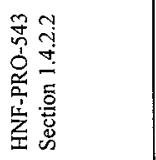 & 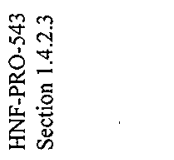 & 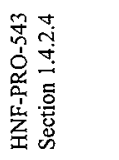 & 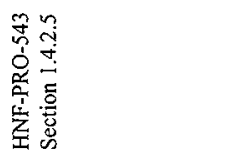 & 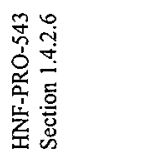 \\
\hline 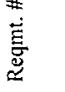 & $\begin{array}{l}\mathbf{J} \\
\stackrel{m}{\infty} \\
\stackrel{m}{n}\end{array}$ & 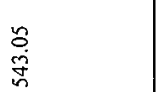 & $\begin{array}{l}\stackrel{8}{0} \\
\text { 市 } \\
\text { W }\end{array}$ & $\begin{array}{l}5 \\
\dot{0} \\
\stackrel{v}{n}\end{array}$ & $\begin{array}{l}\infty \\
\stackrel{\infty}{1} \\
\stackrel{9}{5}\end{array}$ & \begin{tabular}{l}
8 \\
8 \\
0 \\
\multirow{n}{n}{}
\end{tabular} \\
\hline
\end{tabular}


TWR-3721 Rev. 0

\begin{tabular}{|c|c|c|c|c|c|c|}
\hline 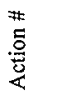 & . & & & & & \\
\hline 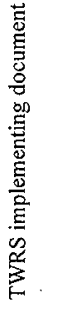 & 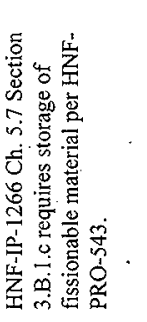 & 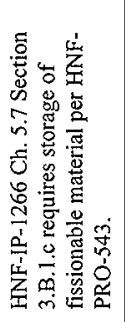 & 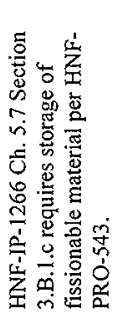 & 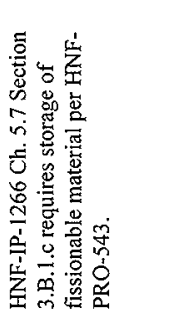 & 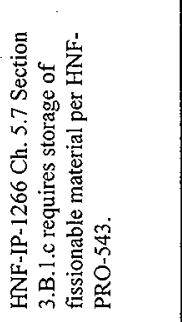 & 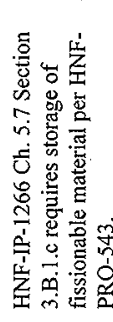 \\
\hline 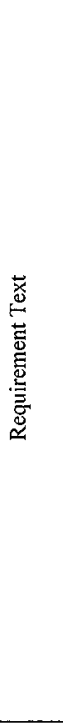 & 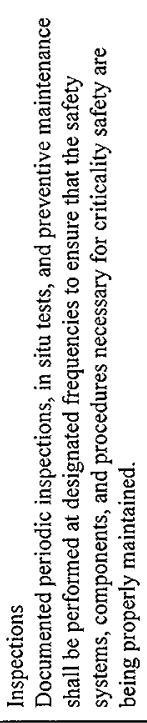 & 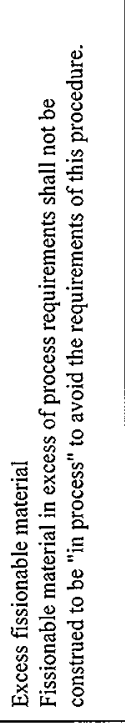 & 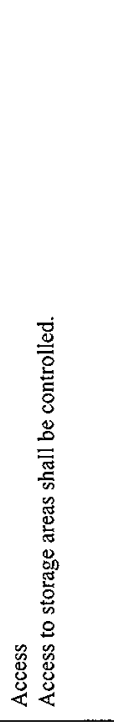 & 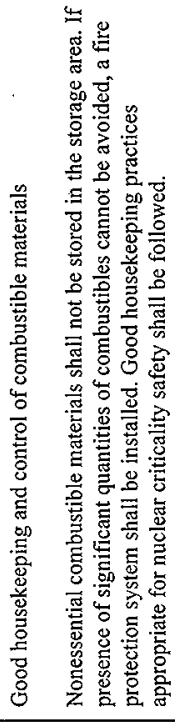 & 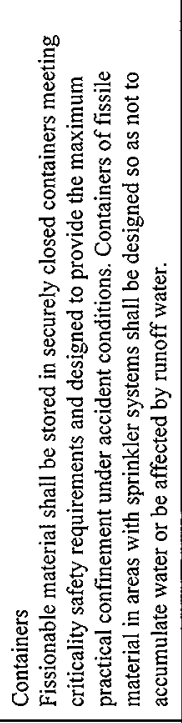 & 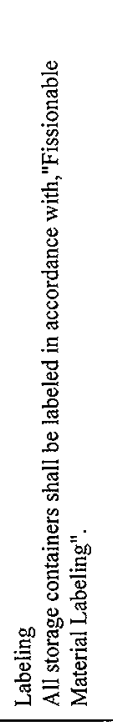 \\
\hline 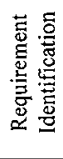 & 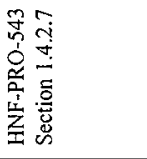 & 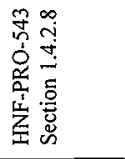 & 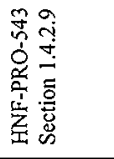 & 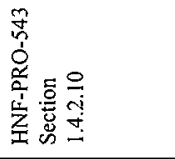 & 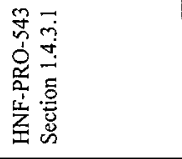 & 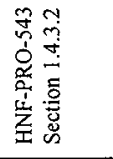 \\
\hline 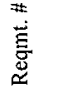 & $\begin{array}{l}\stackrel{\circ}{\circ} \\
\text { 昜 }\end{array}$ & $\begin{array}{l}\bar{m} \\
\vec{d}\end{array}$ & $\frac{9}{0}$ & $\frac{m}{\vec{n}}$ & $\begin{array}{l}\frac{\forall}{\infty} \\
\dot{g}\end{array}$ & $\frac{n}{\frac{g}{b}}$ \\
\hline
\end{tabular}


TWR-3721 Rev. 0

\begin{tabular}{|c|c|c|c|c|c|c|}
\hline 華 & & & & & & \\
\hline 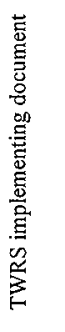 & 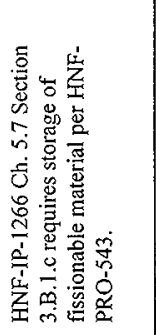 & 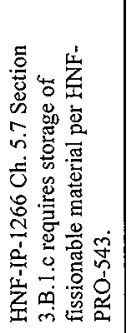 & 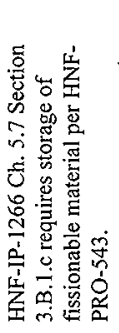 & 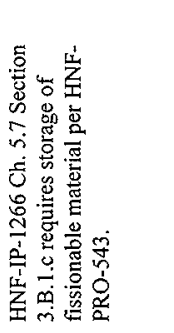 & 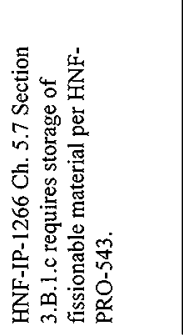 & 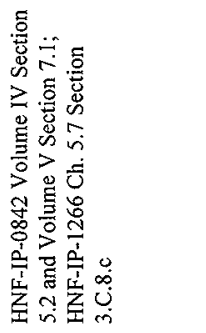 \\
\hline 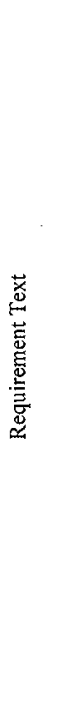 & 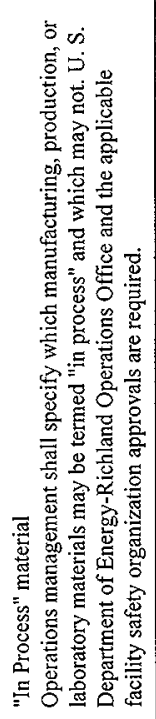 & 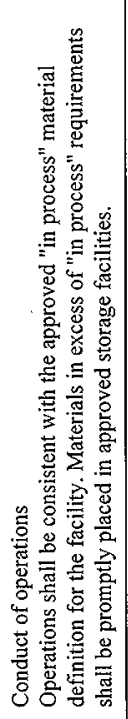 & 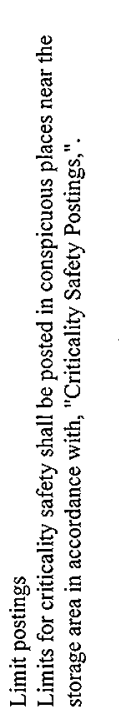 & 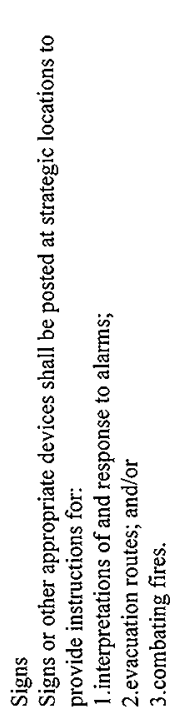 & 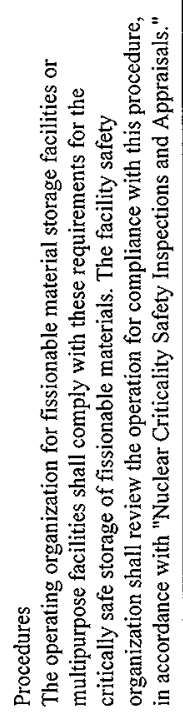 & 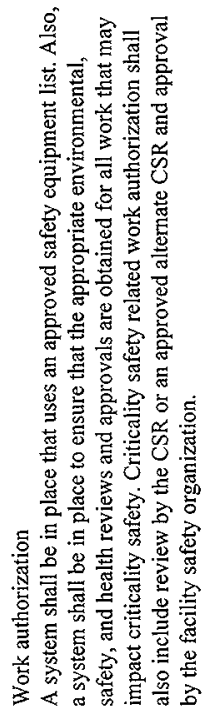 \\
\hline 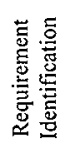 & 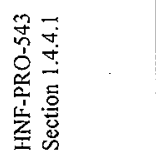 & 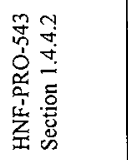 & 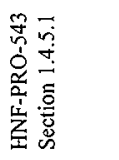 & 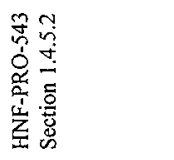 & 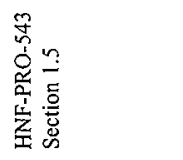 & 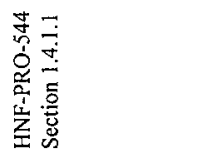 \\
\hline 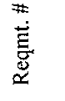 & 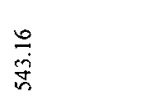 & $\begin{array}{l} \pm \\
\stackrel{m}{n}\end{array}$ & $\frac{\infty}{\stackrel{\infty}{=}}$ & $\frac{9}{\frac{m}{5}}$ & $\begin{array}{l}\stackrel{\oplus}{y} \\
\ddot{*}\end{array}$ & $\begin{array}{l}\text { Do } \\
\text { 莴 }\end{array}$ \\
\hline
\end{tabular}


TWR-3721 Rev. 0

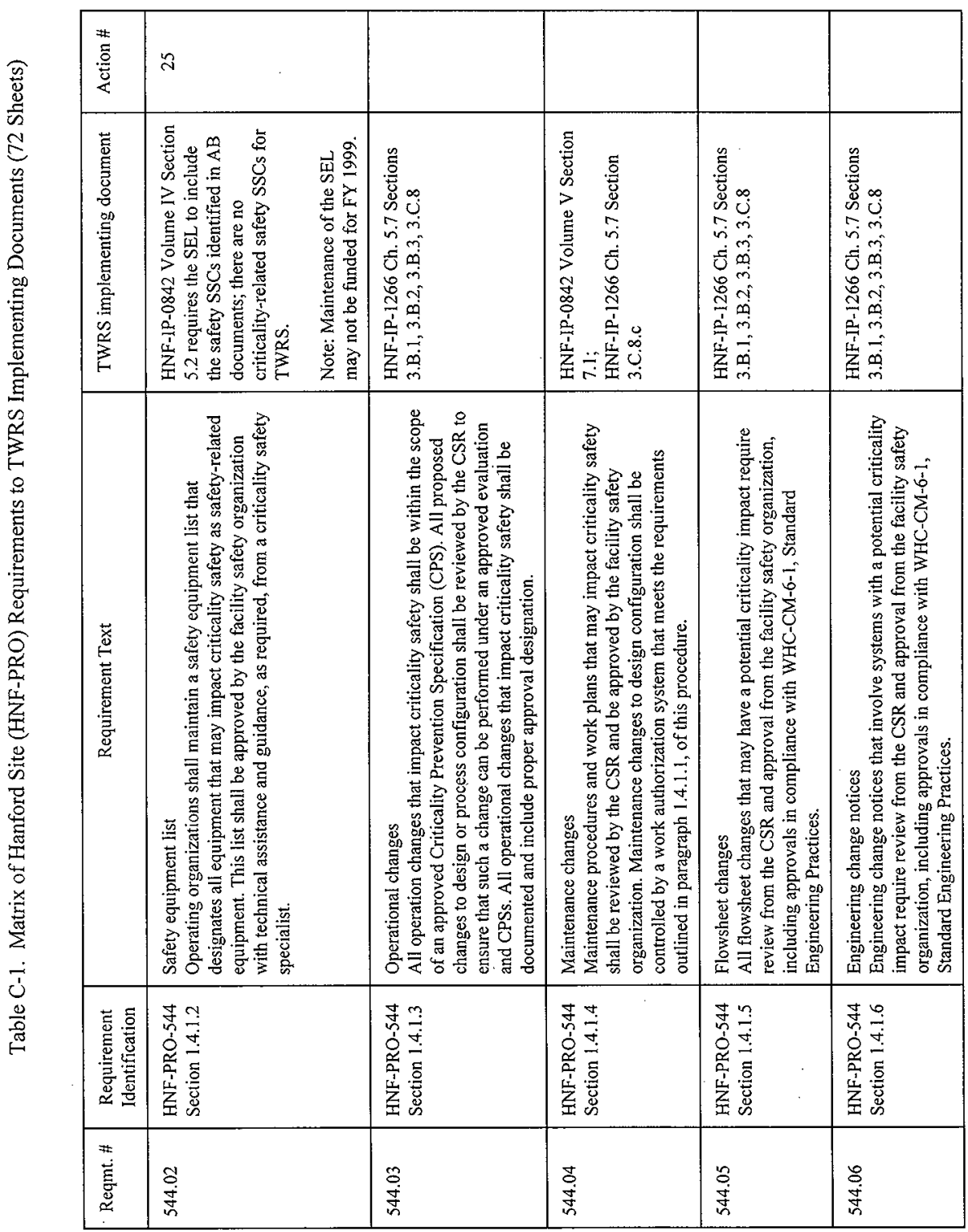


TWR-3721 Rev. 0

\begin{tabular}{|c|c|c|c|c|c|}
\hline 范 & & 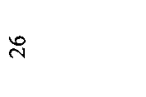 & & \multicolumn{2}{|l|}{$\tilde{N}$} \\
\hline 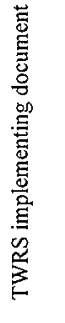 & 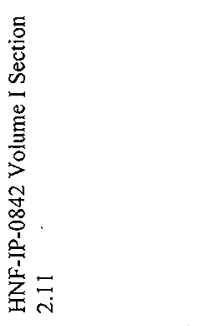 & 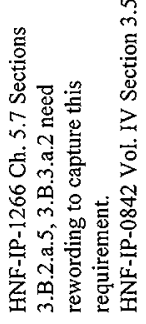 & 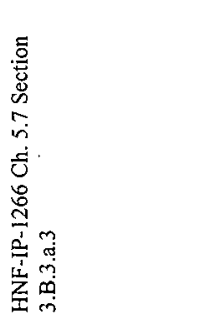 & \multicolumn{2}{|l|}{ 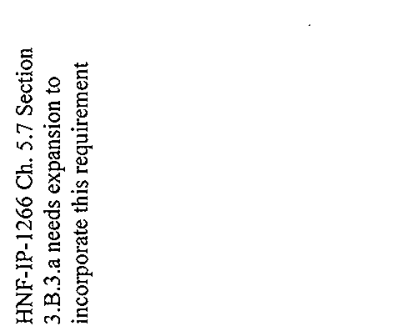 } \\
\hline 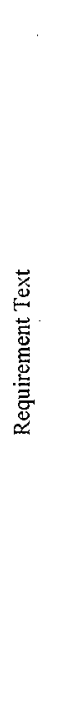 & 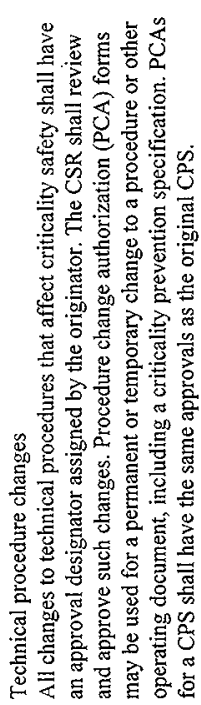 & 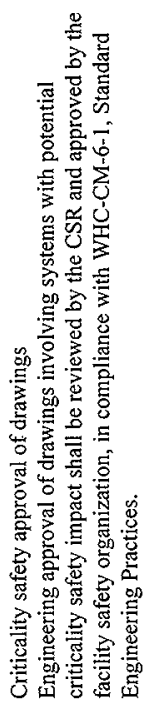 & 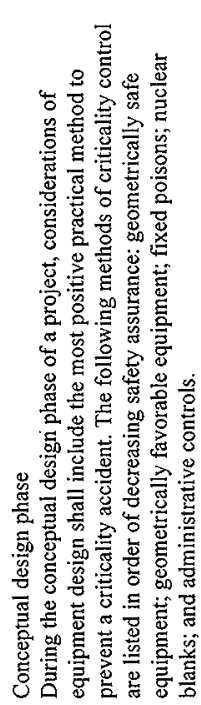 & 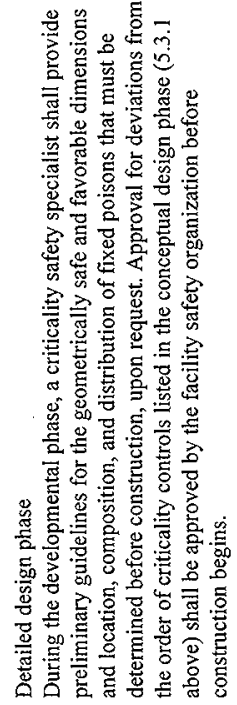 & 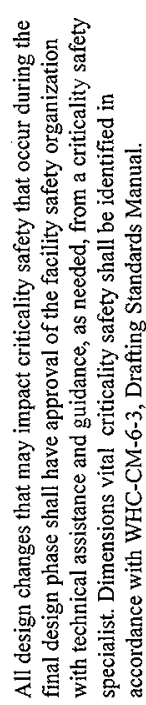 \\
\hline 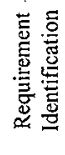 & 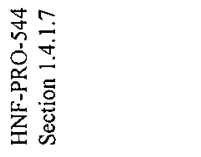 & 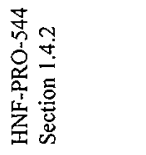 & 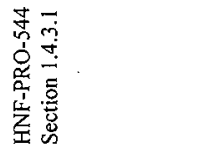 & 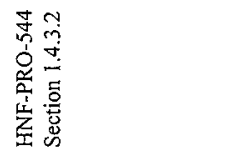 & \\
\hline 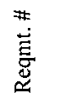 & $\begin{array}{l}5 \\
\text { 守 } \\
\text { in }\end{array}$ & $\begin{array}{l}\infty \\
\stackrel{\infty}{0} \\
\text { 号 }\end{array}$ & 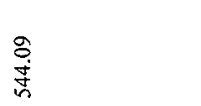 & 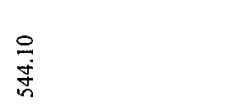 & \\
\hline
\end{tabular}

Page C-58 
TWR-3721 Rev. 0

\begin{tabular}{|c|c|c|c|c|c|c|}
\hline 䜦 & $\stackrel{\infty}{\sim}$ & . & ले & 이 & & \\
\hline 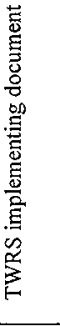 & & 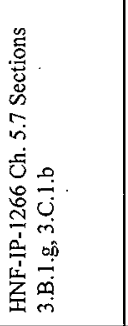 & 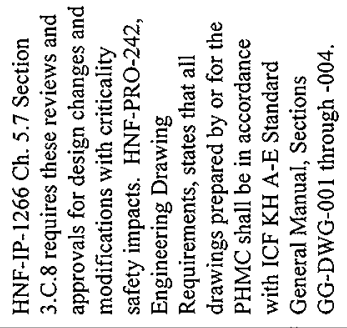 & 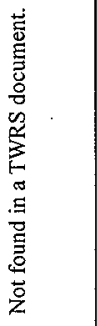 & 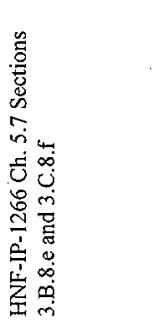 & \\
\hline 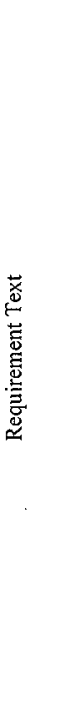 & 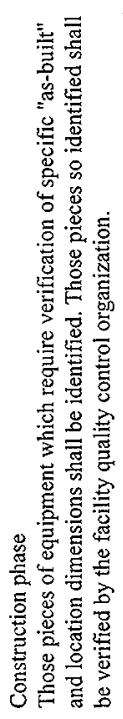 & 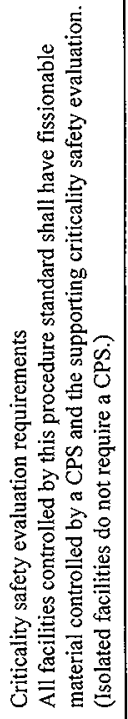 & 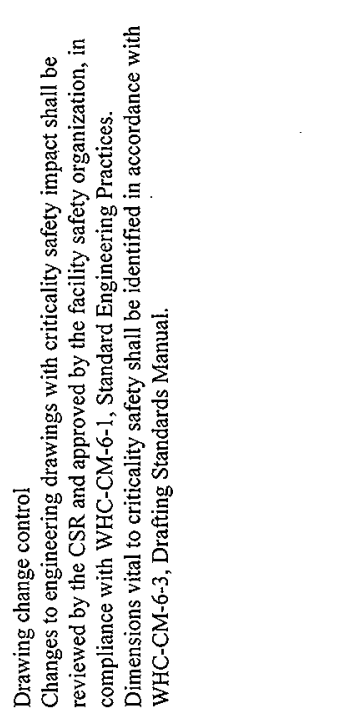 & 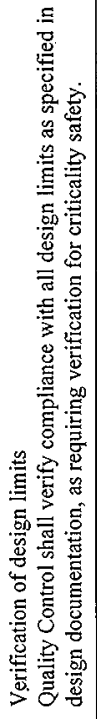 & 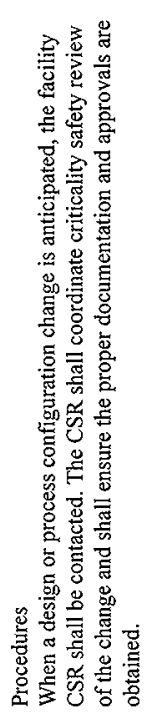 & 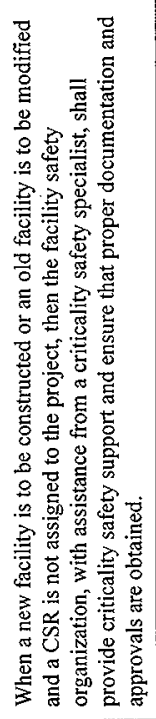 \\
\hline 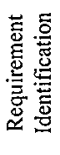 & 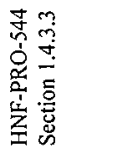 & 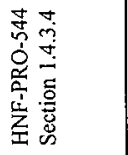 & 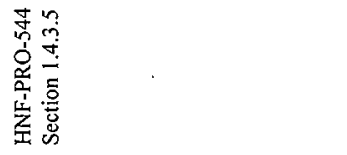 & 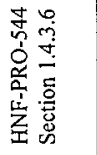 & 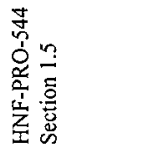 & \\
\hline 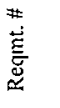 & $\begin{array}{l}= \\
\text { 导 }\end{array}$ & 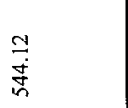 & $\frac{m}{j}$ & $\begin{array}{l} \pm \\
\stackrel{ \pm}{ \pm}\end{array}$ & $\begin{array}{l}\frac{n}{j} \\
\frac{j}{b}\end{array}$ & \\
\hline
\end{tabular}

Page C-59 
TWR-3721 Rev. 0

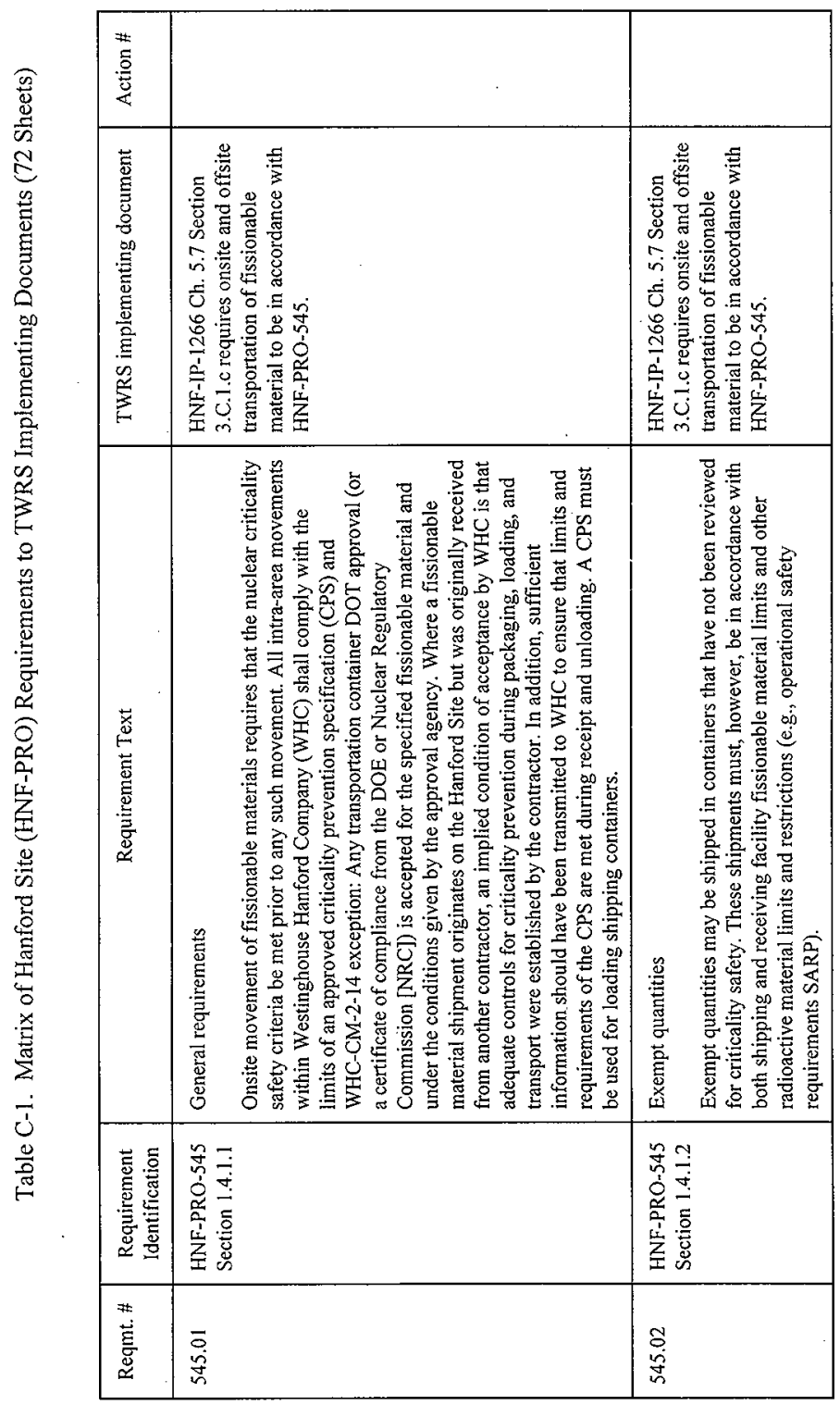


TWR-3721 Rev. 0

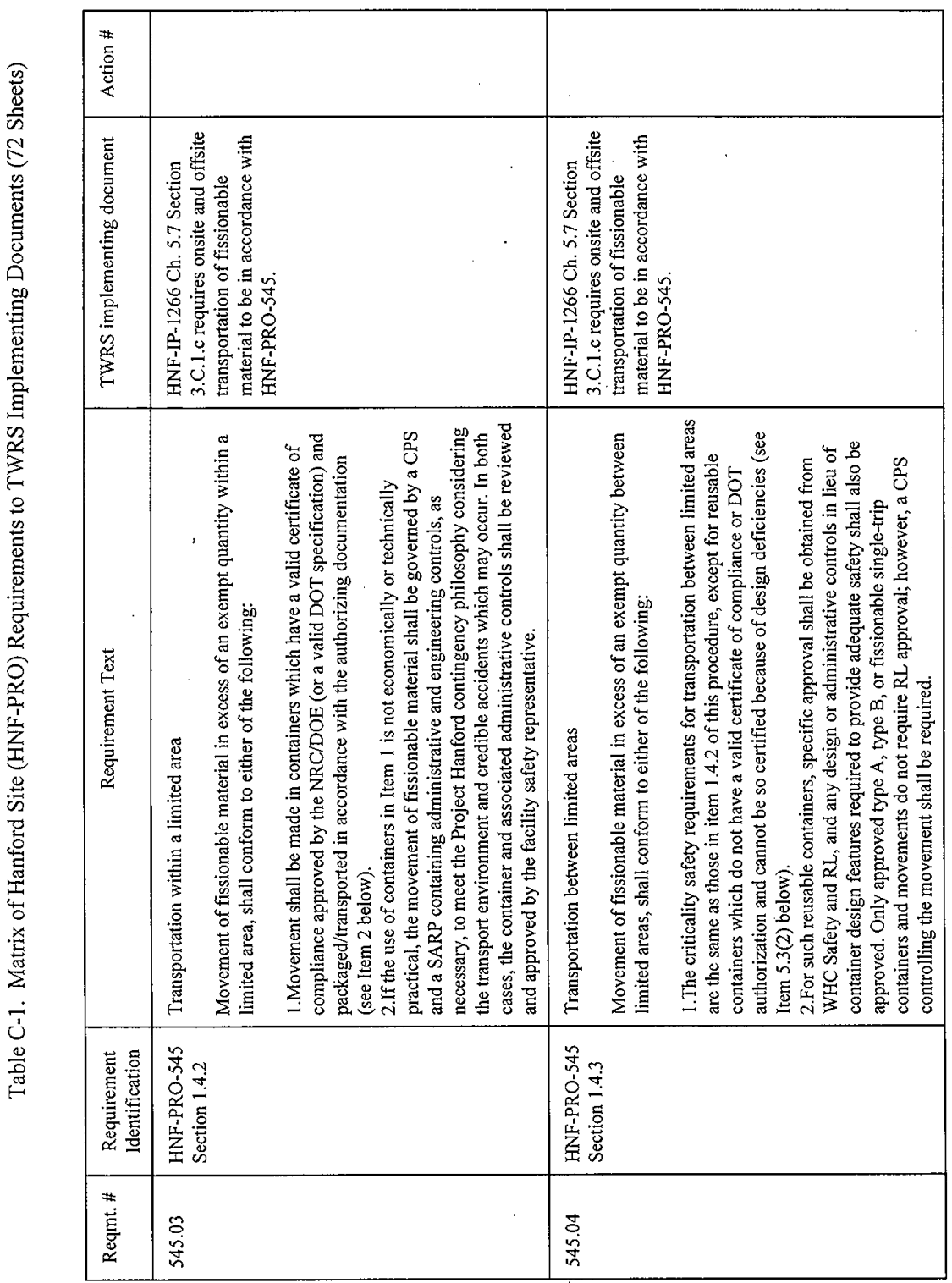


TWR-3721 Rev. 0

\begin{tabular}{|c|c|c|c|c|c|c|}
\hline 泀 & & & & & & \\
\hline 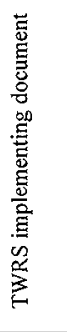 & 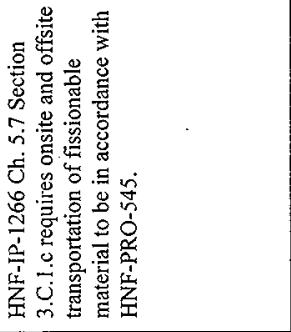 & 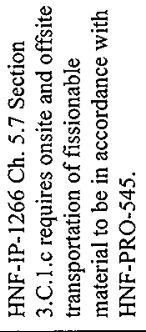 & 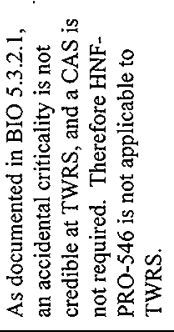 & 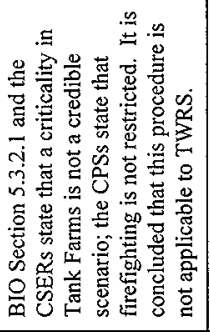 & 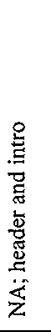 & \\
\hline 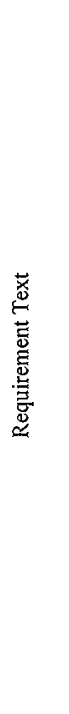 & 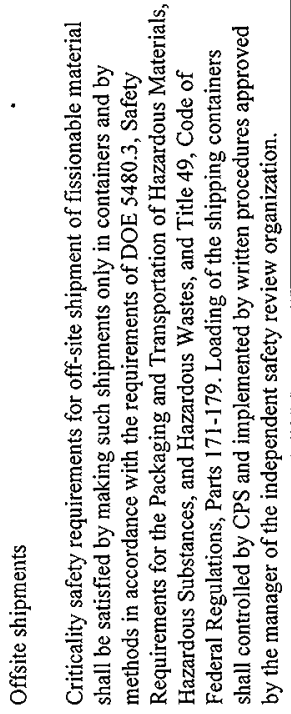 & 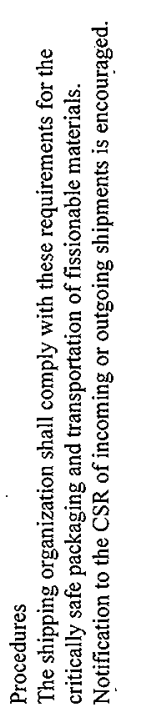 & 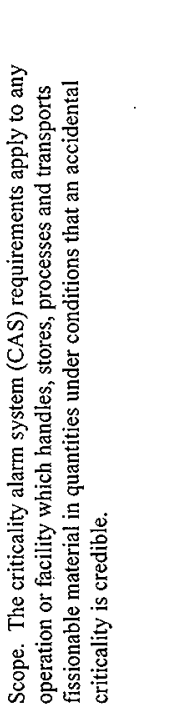 & 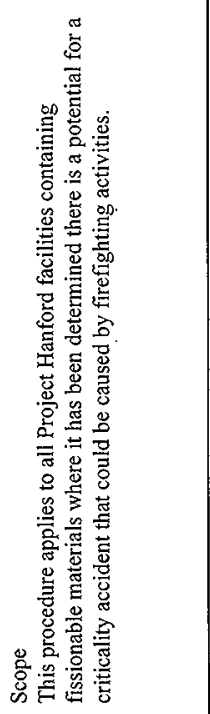 & 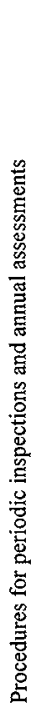 & 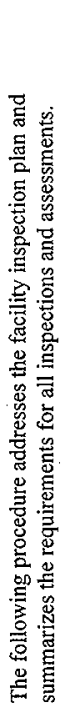 \\
\hline 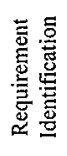 & 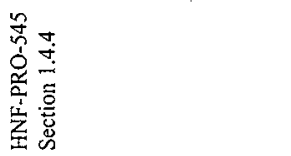 & 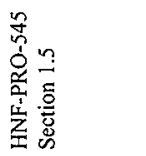 & 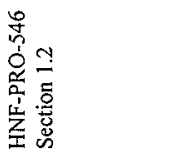 & 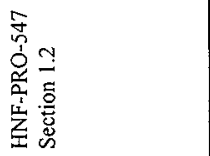 & 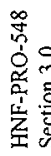 & \\
\hline 苾 & $\begin{array}{l}\stackrel{8}{8} \\
\stackrel{2}{n}\end{array}$ & $\begin{array}{l}\stackrel{8}{\circ} \\
\text { in } \\
\text { ज́ }\end{array}$ & $\begin{array}{l}\bar{\sigma} \\
\text { : } \\
\text { H. }\end{array}$ & $\underset{\text { S }}{\stackrel{5}{*}}$ & $\begin{array}{l}\overrightarrow{0} \\
\infty \\
\text { 芦 }\end{array}$ & \\
\hline
\end{tabular}

Page C-62 
TWR-3721 Rev. 0

\begin{tabular}{|c|c|c|c|c|c|c|c|c|c|c|c|}
\hline 莡 & & \multicolumn{4}{|l|}{$\bar{m}$} & \multicolumn{3}{|l|}{$\vec{m}$} & \multicolumn{3}{|l|}{$\vec{m}$} \\
\hline 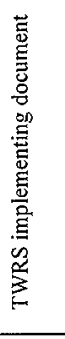 & 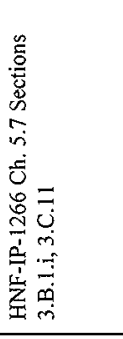 & \multicolumn{4}{|c|}{ 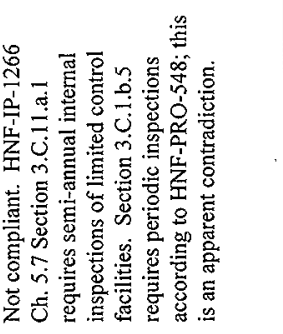 } & \multicolumn{3}{|c|}{ 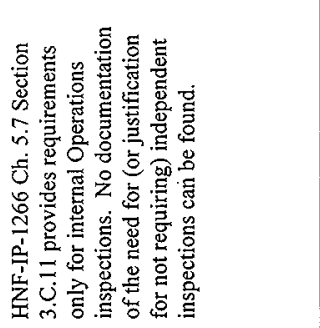 } & \multicolumn{3}{|c|}{ 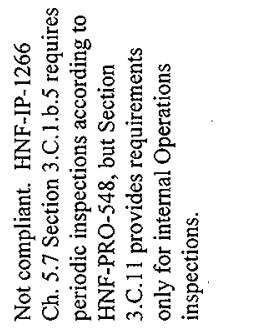 } \\
\hline 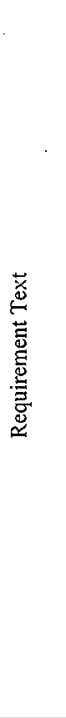 & 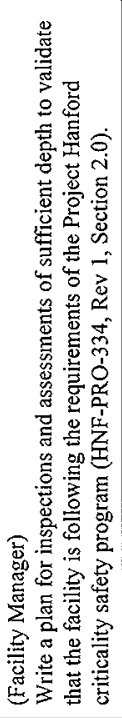 & 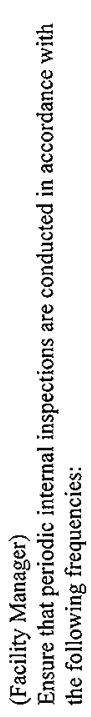 & 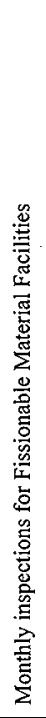 & 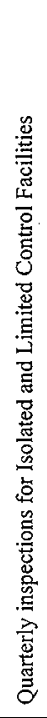 & 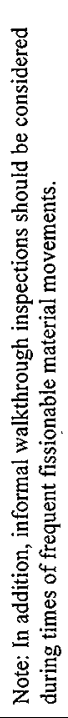 & 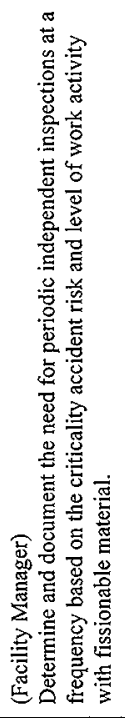 & 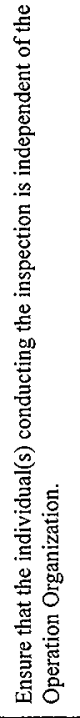 & 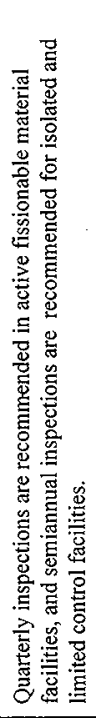 & 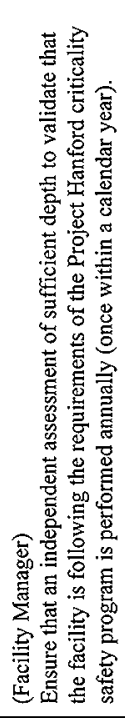 & 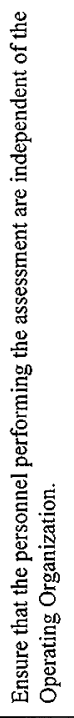 & 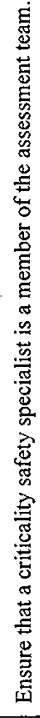 \\
\hline 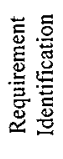 & 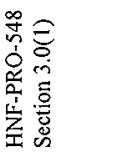 & 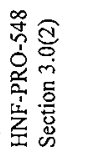 & & & & 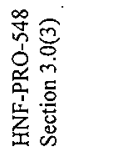 & & & 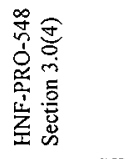 & & \\
\hline $\begin{array}{l}\text { 華 } \\
\dot{\vec{E}} \\
\ddot{\vec{E}}\end{array}$ & \begin{tabular}{l}
$\stackrel{8}{0}$ \\
o \\
\multirow{4}{n}{}
\end{tabular} & $\begin{array}{l}8 \\
8 \\
00 \\
\text { in }\end{array}$ & & & & 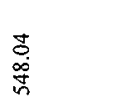 & & & $\begin{array}{l}0 \\
\infty \\
\infty \\
0 \\
0\end{array}$ & & \\
\hline
\end{tabular}


TWR-3721 Rev. 0

\begin{tabular}{|c|c|c|c|c|c|c|}
\hline 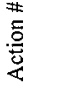 & $\vec{m}$ & & $\bar{n}$ & $\bar{m}$ & $\bar{m}$ & $\bar{m}$ \\
\hline 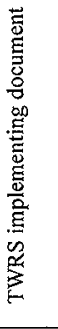 & & 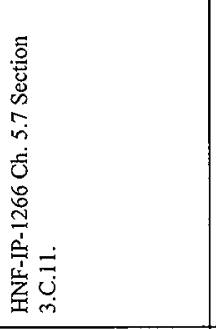 & 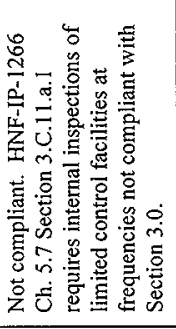 & 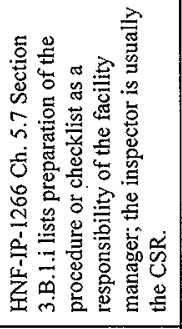 & & \\
\hline 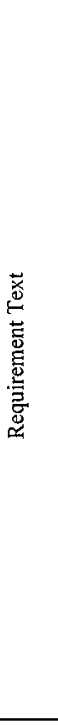 & 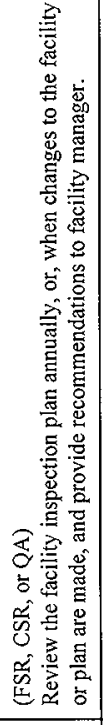 & 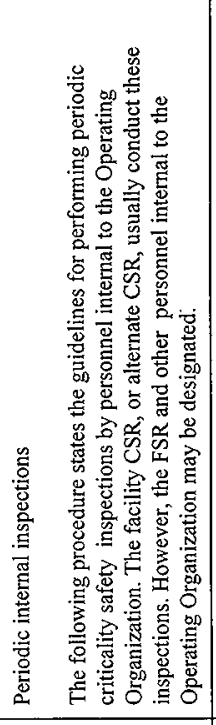 & 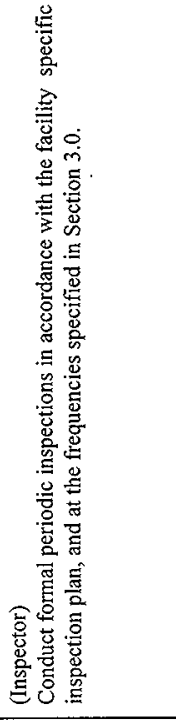 & 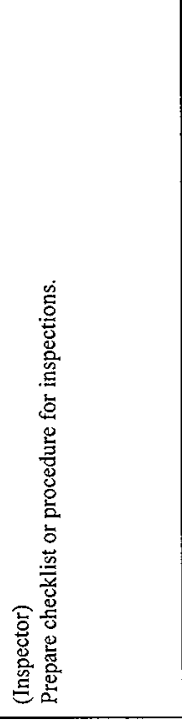 & 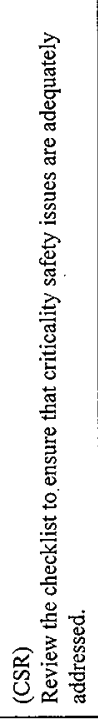 & 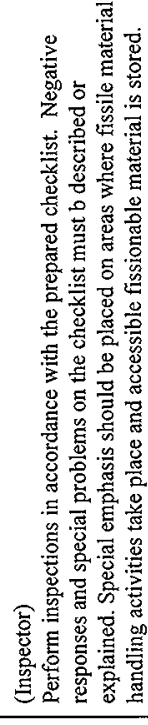 \\
\hline 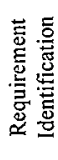 & 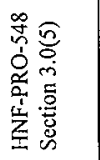 & 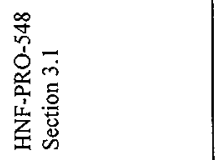 & 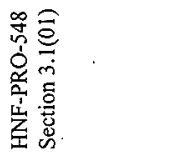 & 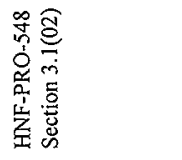 & 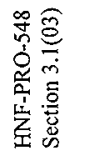 & 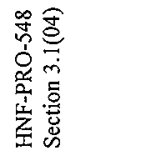 \\
\hline $\begin{array}{l}\text { \# } \\
\stackrel{\vec{E}}{\vec{E}} \\
\stackrel{4}{\varkappa}\end{array}$ & $\begin{array}{l}8 \\
8 \\
\infty \\
0 \\
0\end{array}$ & 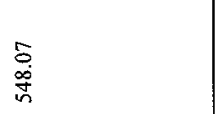 & 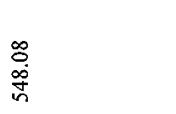 & $\begin{array}{l}\text { o } \\
\text { co } \\
\text { of }\end{array}$ & $\begin{array}{l}\stackrel{0}{0} \\
\text { ơ } \\
\end{array}$ & $\begin{array}{l}\overline{=} \\
\text { o } \\
\vdots\end{array}$ \\
\hline
\end{tabular}


TWR-3721 Rev. 0

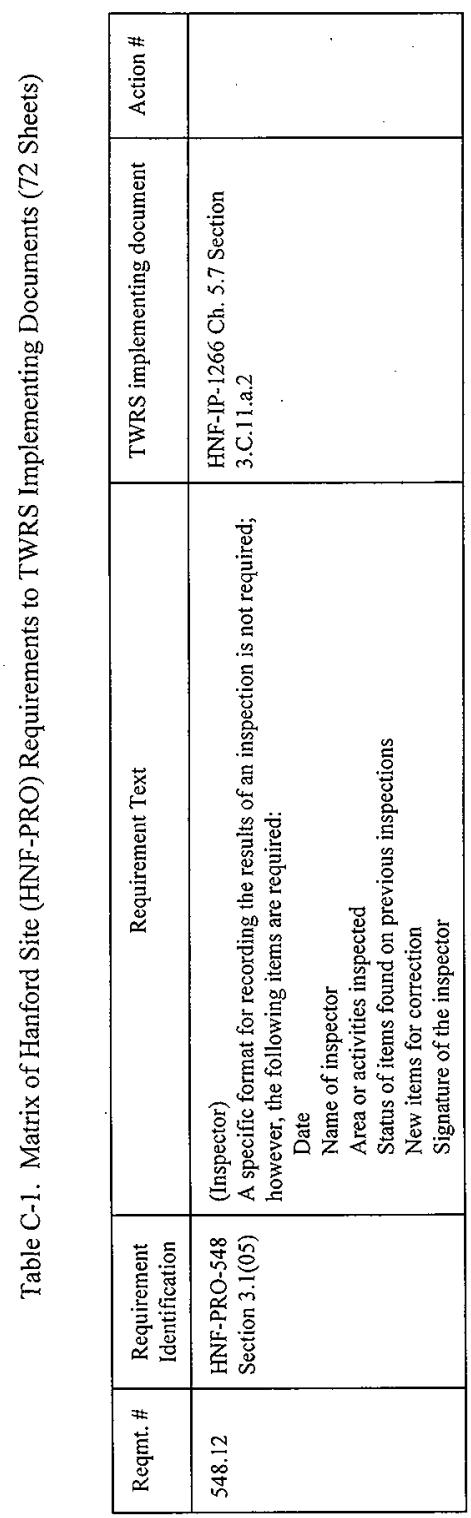

Page C-65 
TWR-3721 Rev. 0

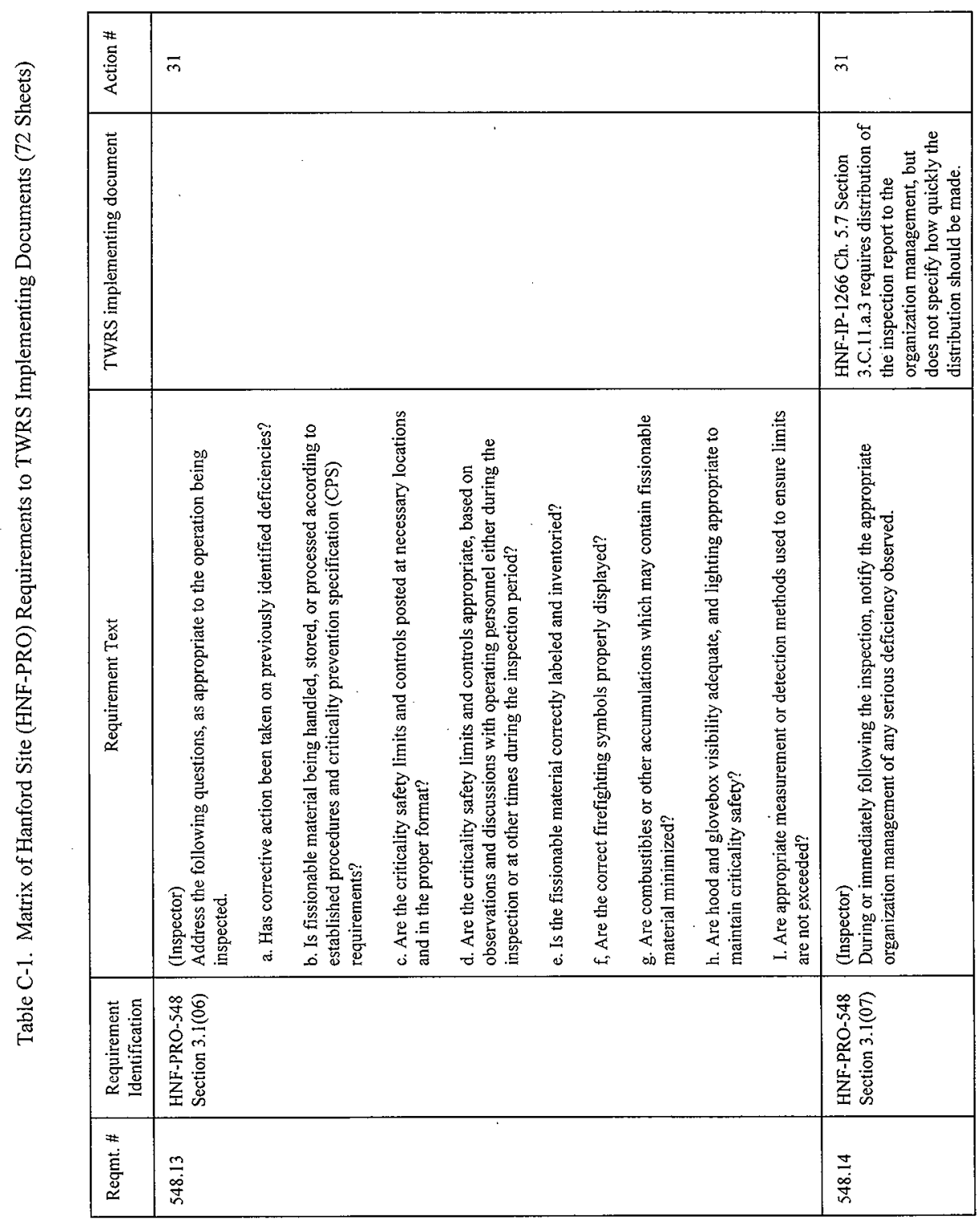

Page C-66 
TWR-3721 Rev. 0

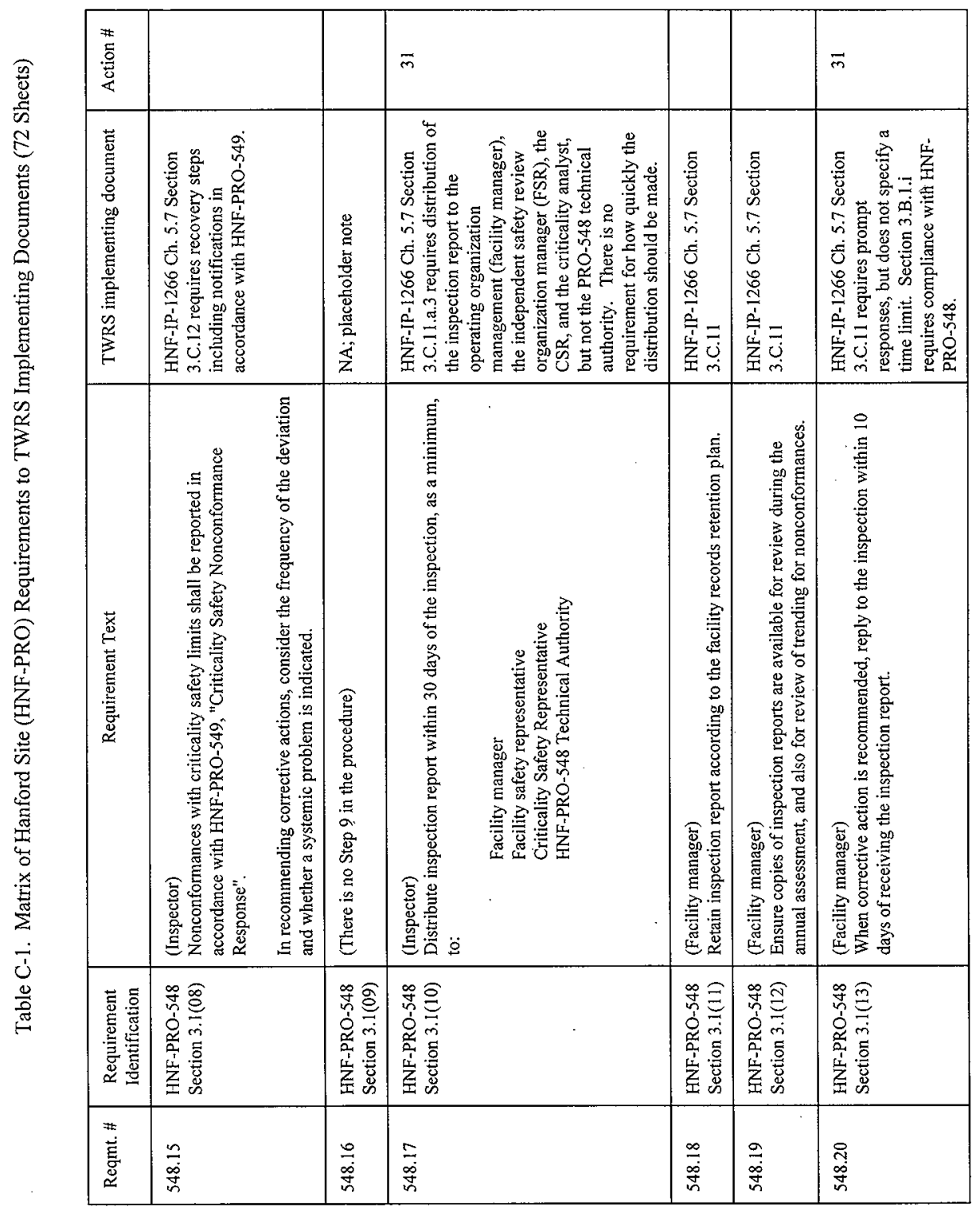


TWR-3721 Rev. 0

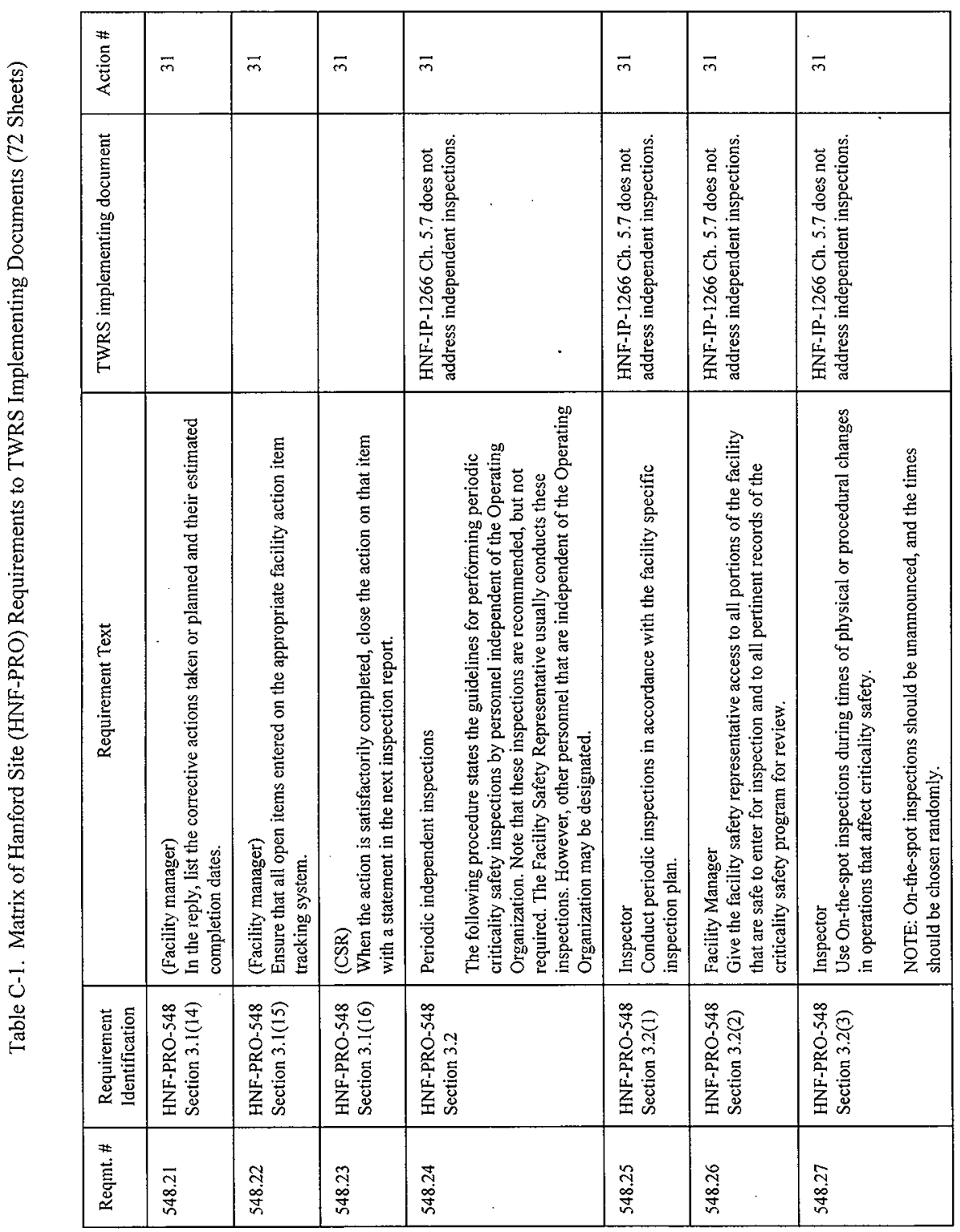


TWR-3721 Rev. 0

\begin{tabular}{|c|c|c|c|c|c|c|c|c|c|}
\hline 节 & $\vec{m}$ & $\bar{m}$ & $\bar{m}$ & $\bar{m}$ & $\bar{m}$ & $\vec{m}$ & $\bar{m}$ & $\bar{m}$ & $\vec{m}$ \\
\hline 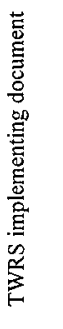 & 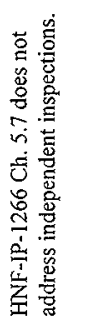 & 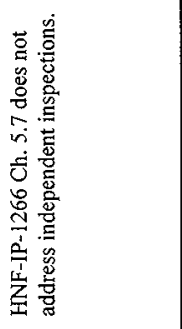 & 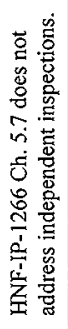 & 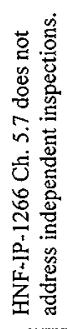 & 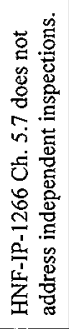 & 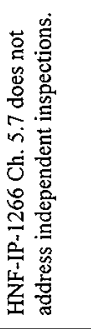 & 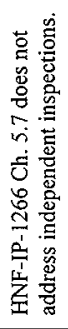 & 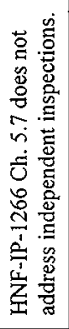 & 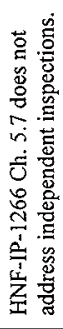 \\
\hline 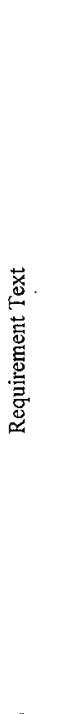 & 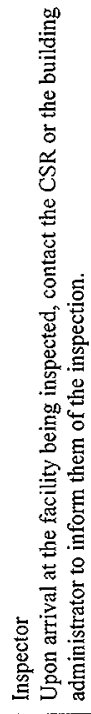 & 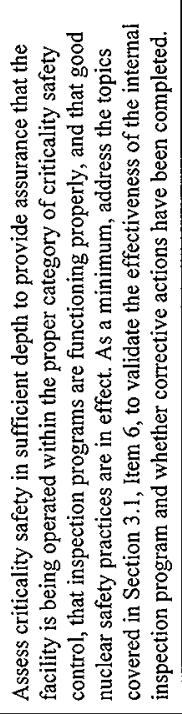 & 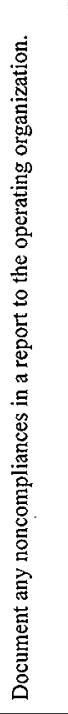 & 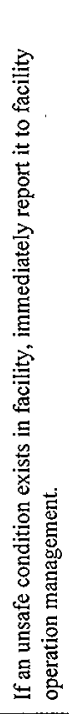 & 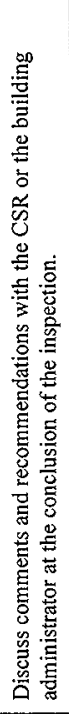 & 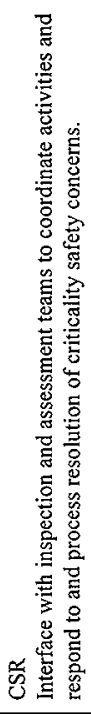 & 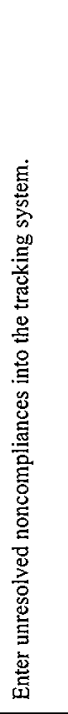 & 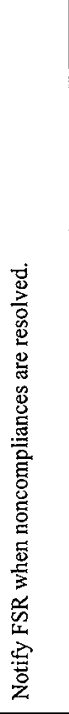 & 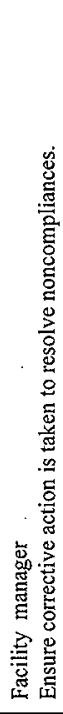 \\
\hline 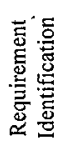 & 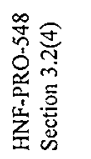 & 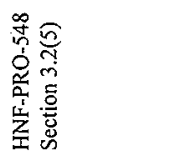 & 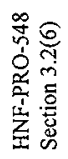 & 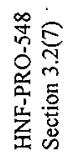 & 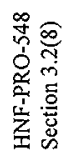 & 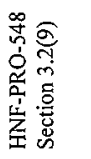 & 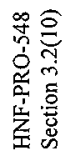 & 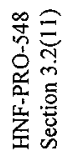 & 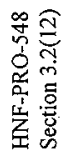 \\
\hline 苛 & 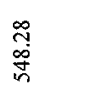 & $\begin{array}{l}\text { ते } \\
\text { of } \\
\text { in }\end{array}$ & 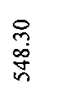 & $\begin{array}{l}\bar{m} \\
\text { o } \\
\text { in }\end{array}$ & 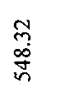 & 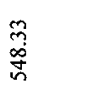 & 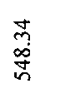 & 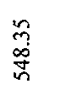 & 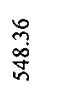 \\
\hline
\end{tabular}


TWR-3721 Rev. 0

\begin{tabular}{|c|c|c|c|c|c|}
\hline 帮 & $\vec{m}$ & $\bar{m}$ & $\vec{n}$ & $\vec{m}$ & $\vec{m}$ \\
\hline 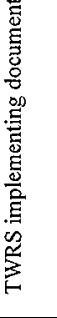 & 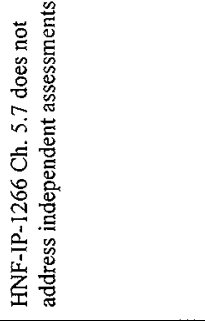 & 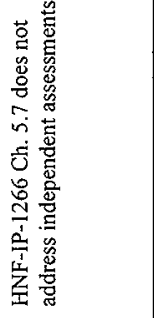 & 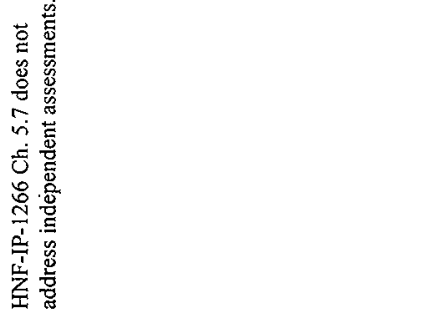 & 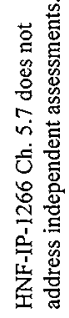 & 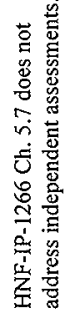 \\
\hline 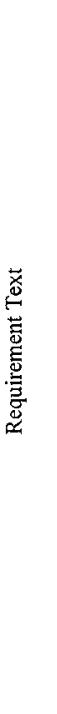 & 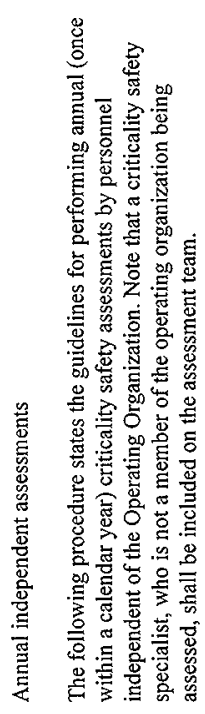 & 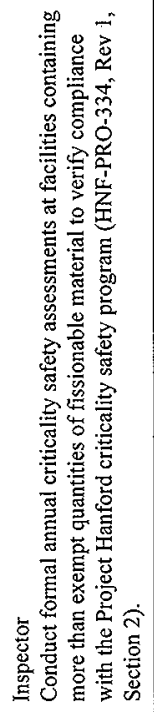 & 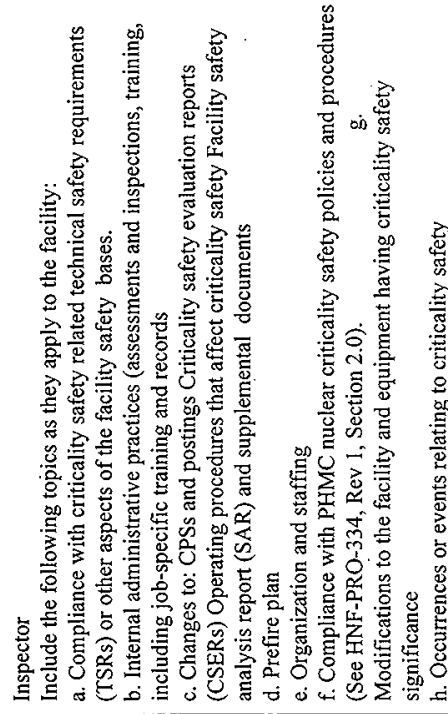 & 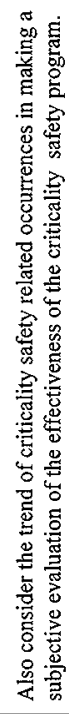 & 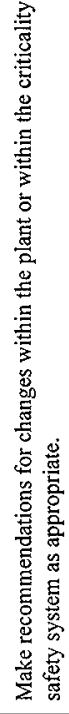 \\
\hline 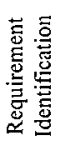 & 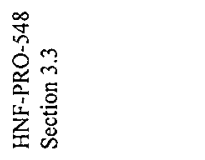 & 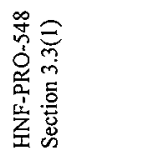 & 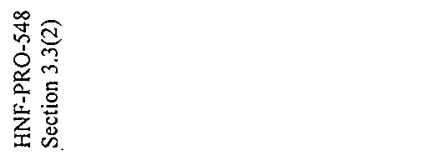 & 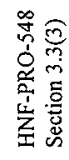 & 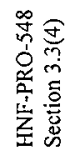 \\
\hline 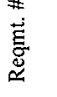 & $\begin{array}{l}\hat{n} \\
0 \\
0 \\
\bar{n}\end{array}$ & 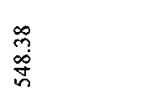 & $\begin{array}{l}\text { के } \\
\text { o } \\
\text { जે }\end{array}$ & $\begin{array}{l}\stackrel{8}{+} \\
\stackrel{\infty}{0} \\
\stackrel{0}{n}\end{array}$ & 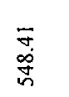 \\
\hline
\end{tabular}


TWR-3721 Rev. 0

\begin{tabular}{|c|c|c|c|c|c|c|c|c|c|}
\hline 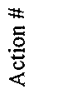 & $\bar{m}$ & & $\bar{m}$ & $\bar{m}$ & $\bar{m}$ & $\bar{m}$ & $\bar{m}$ & $\bar{n}$ & $\mathscr{r}$ \\
\hline 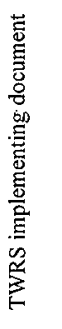 & 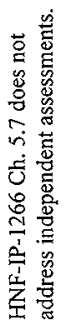 & & 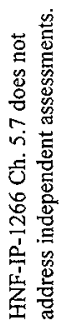 & 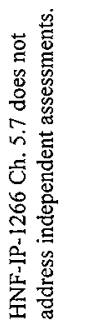 & 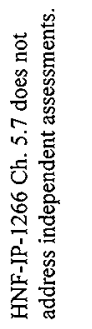 & 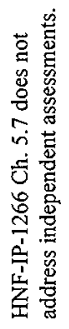 & 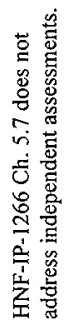 & 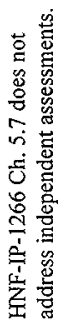 & 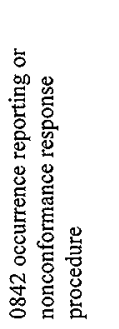 \\
\hline 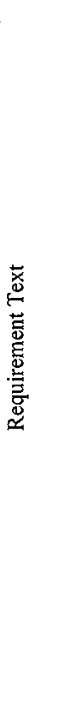 & 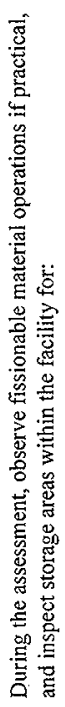 & 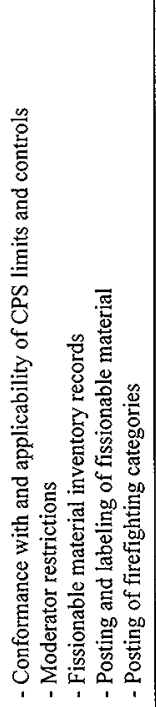 & 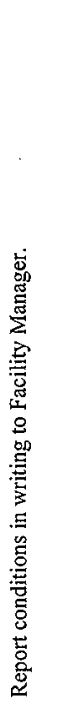 & 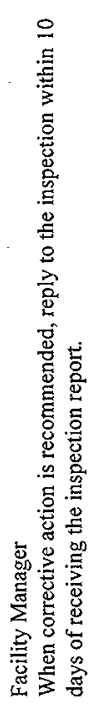 & 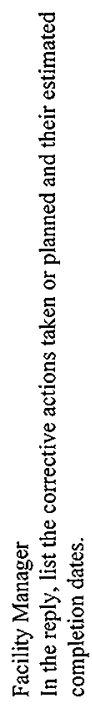 & 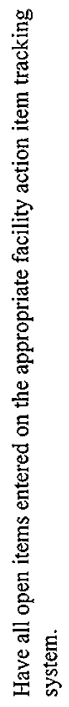 & 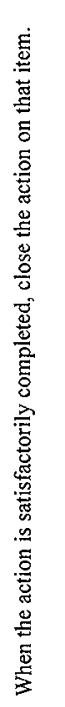 & 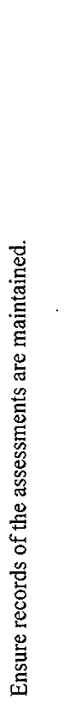 & 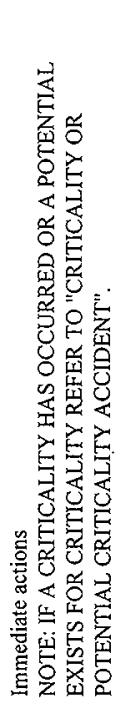 \\
\hline 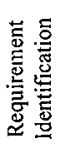 & 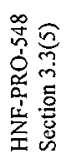 & & 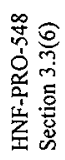 & 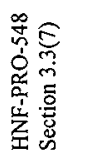 & 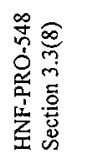 & 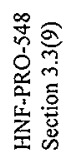 & 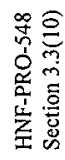 & 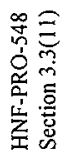 & 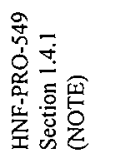 \\
\hline 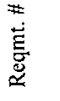 & $\begin{array}{l}\text { Fे } \\
\text { D } \\
\text { S }\end{array}$ & & $\begin{array}{l}\stackrel{y}{\forall} \\
\text { D } \\
\dot{f}\end{array}$ & 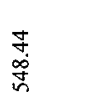 & $\begin{array}{l}\stackrel{2}{*} \\
\stackrel{\infty}{6} \\
\vec{n}\end{array}$ & $\begin{array}{l}\text { Oे } \\
\infty \\
\text { b }\end{array}$ & $\begin{array}{l}\text { 吾 } \\
\infty \\
\vec{f}\end{array}$ & 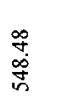 & $\begin{array}{l}\bar{\alpha} \\
\stackrel{0}{0} \\
\dot{B}\end{array}$ \\
\hline
\end{tabular}


TWR-3721 Rev. 0

\begin{tabular}{|c|c|c|c|c|c|c|c|}
\hline 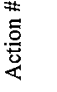 & \multicolumn{5}{|l|}{ ల్ల } & $\tilde{N}$ & ले \\
\hline 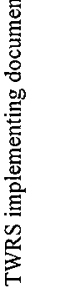 & \multicolumn{5}{|c|}{ 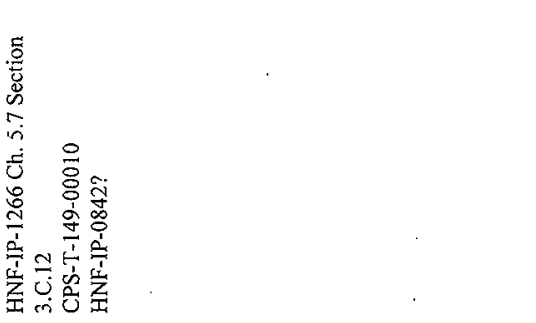 } & 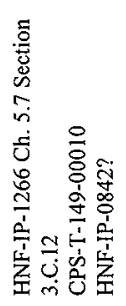 & 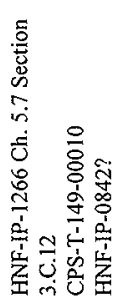 \\
\hline 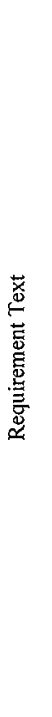 & 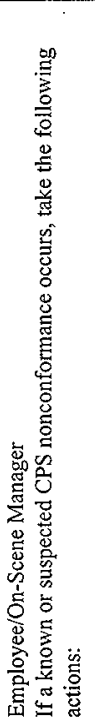 & 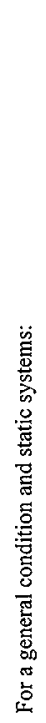 & 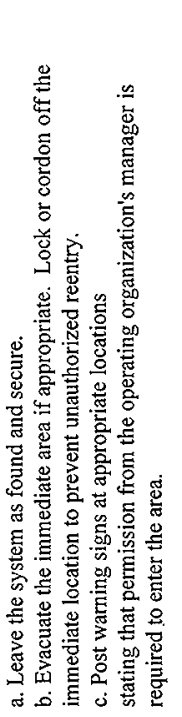 & 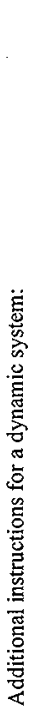 & 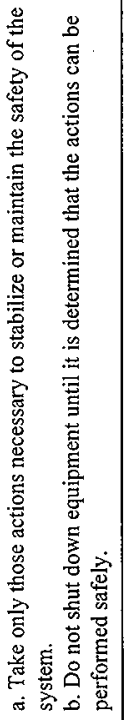 & 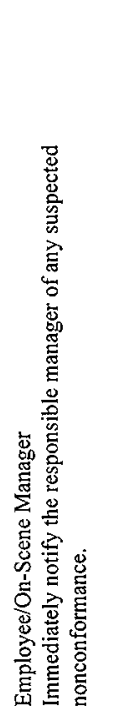 & 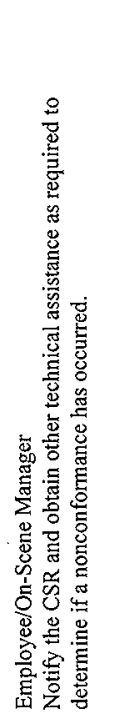 \\
\hline 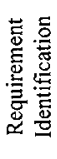 & 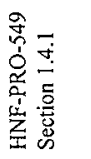 & & & & & 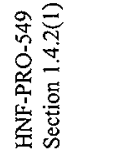 & 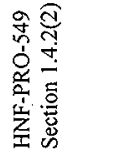 \\
\hline 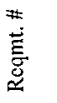 & $\begin{array}{l}\tilde{o} \\
\stackrel{\circ}{\circ}\end{array}$ & & & & & $\begin{array}{l}m \\
\stackrel{3}{\dot{g}} \\
\stackrel{5}{5}\end{array}$ & 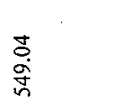 \\
\hline
\end{tabular}

Page C-72 
TWR-3721 Rev. 0

\begin{tabular}{|c|c|c|c|c|c|c|}
\hline 总 & \multicolumn{2}{|l|}{ ले } & $\widetilde{m}$ & $\approx$ & $\widetilde{m}$ & है \\
\hline 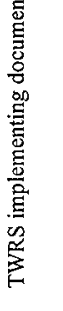 & \multicolumn{2}{|c|}{ 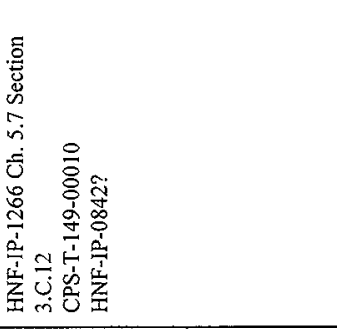 } & 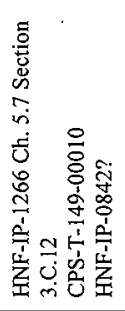 & 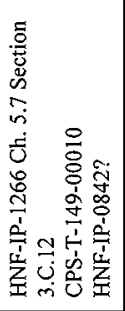 & 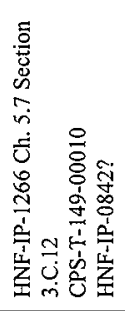 & 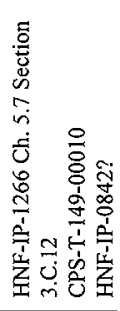 \\
\hline 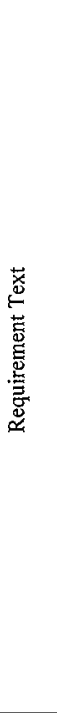 & 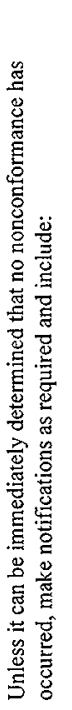 & 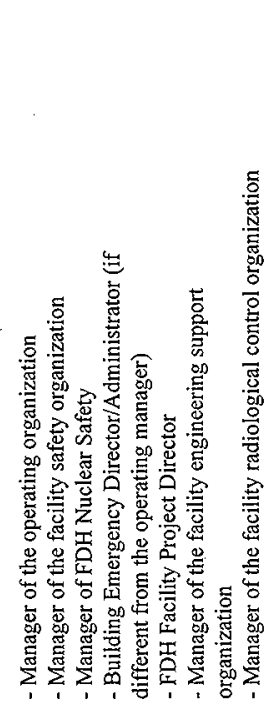 & 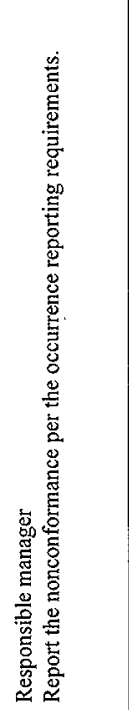 & 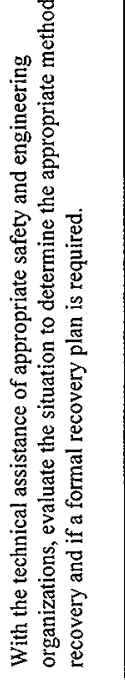 & 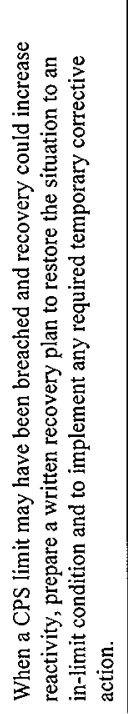 & 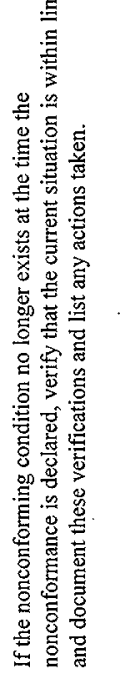 \\
\hline 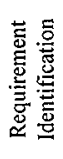 & 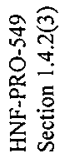 & & 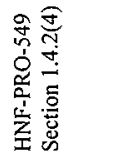 & 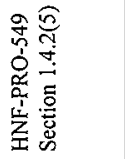 & 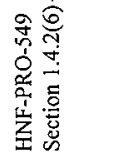 & 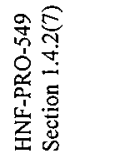 \\
\hline 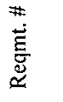 & 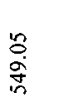 & & $\begin{array}{l}8 \\
\stackrel{0}{0} \\
\text { : }\end{array}$ & $\begin{array}{l}\delta \\
\dot{a} \\
\dot{\sigma}\end{array}$ & $\begin{array}{l}\stackrel{\circ}{\circ} \\
\stackrel{0}{0}\end{array}$ & 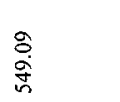 \\
\hline
\end{tabular}

Page C-73 
TWR-3721 Rev. 0

\begin{tabular}{|c|c|c|c|c|c|c|c|}
\hline 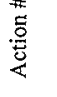 & लै & స్ల & లె & \multicolumn{2}{|l|}{ ల్ } & ले & 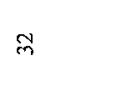 \\
\hline 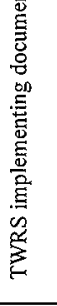 & 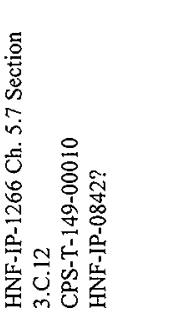 & 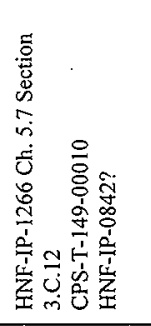 & 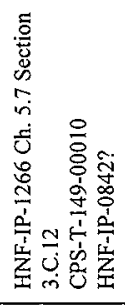 & \multicolumn{2}{|c|}{ 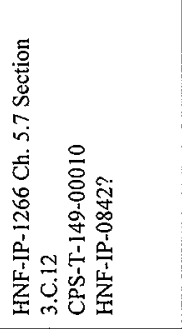 } & 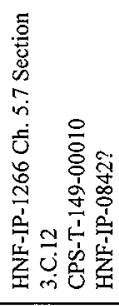 & 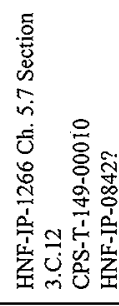 \\
\hline 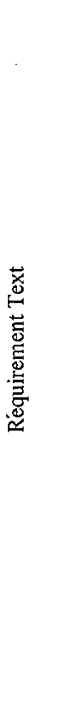 & 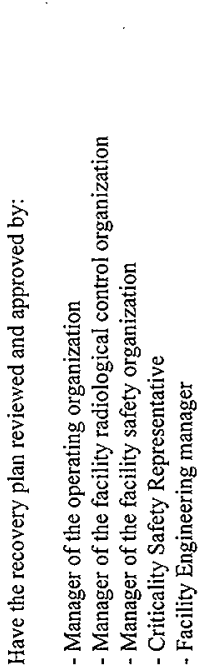 & 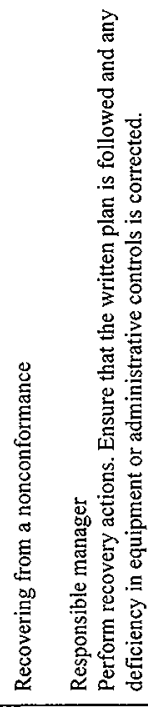 & 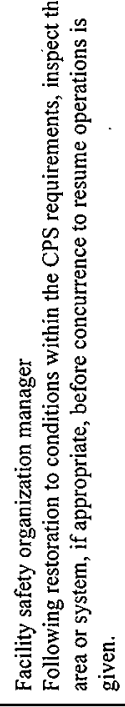 & 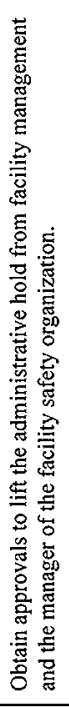 & 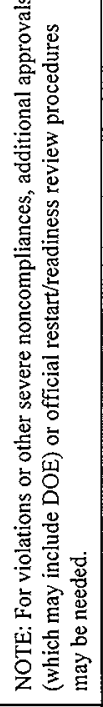 & 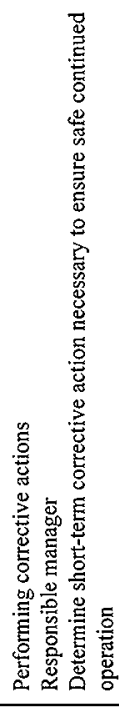 & 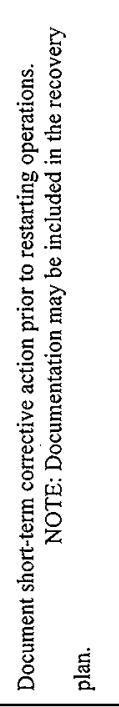 \\
\hline 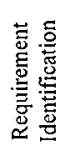 & 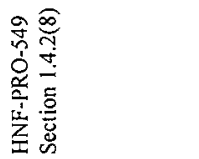 & 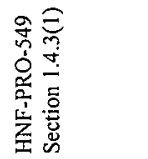 & 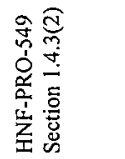 & 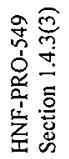 & & 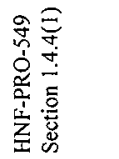 & 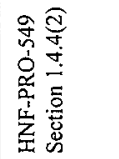 \\
\hline 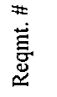 & $\begin{array}{l}\text { 음 } \\
\text { 高 }\end{array}$ & $\begin{array}{l}\overrightarrow{0} \\
\overrightarrow{0}\end{array}$ & $\frac{a}{\square}$ & $\frac{m}{9}$ & & 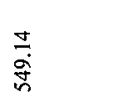 & $\stackrel{n}{\stackrel{a}{g}}$ \\
\hline
\end{tabular}


TWR-3721 Rev. 0

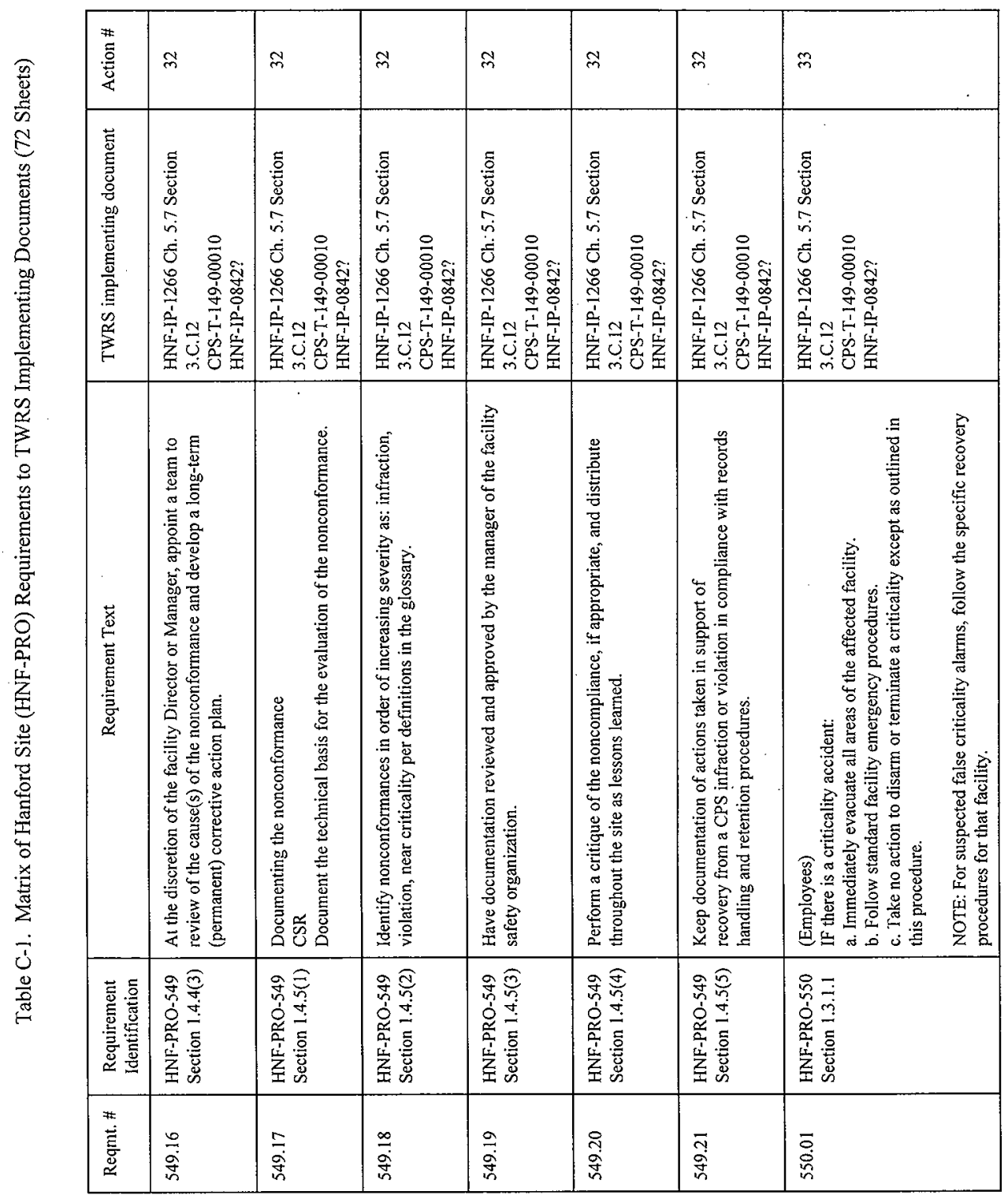


TWR-3721 Rev. 0

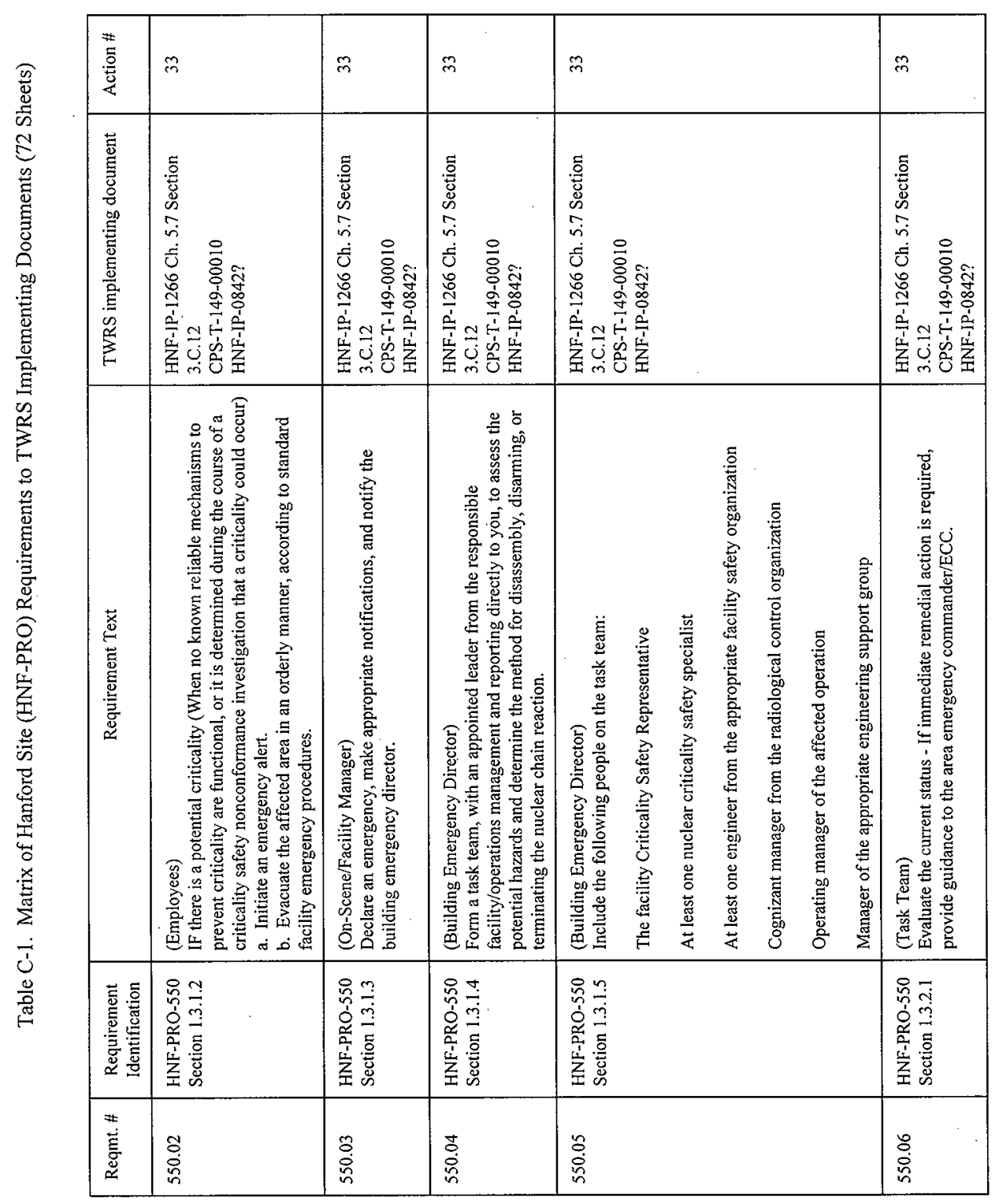

Page C-76 
TWR-3721 Rev. 0

\begin{tabular}{|c|c|c|c|c|c|c|}
\hline 范 & \multicolumn{2}{|l|}{$m$} & 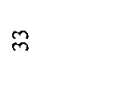 & $m$ & $\stackrel{m}{m}$ & $\stackrel{m}{m}$ \\
\hline 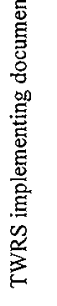 & \multicolumn{2}{|c|}{ 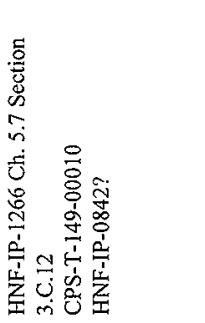 } & 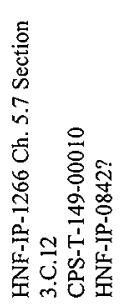 & 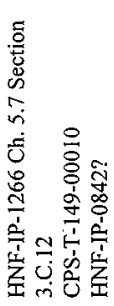 & 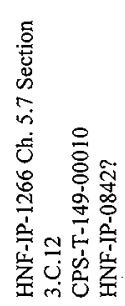 & 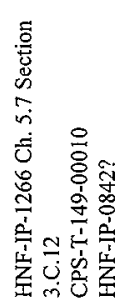 \\
\hline 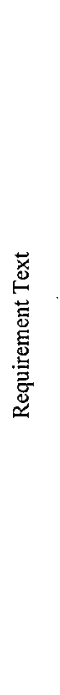 & 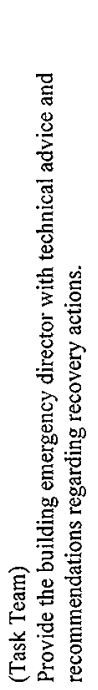 & 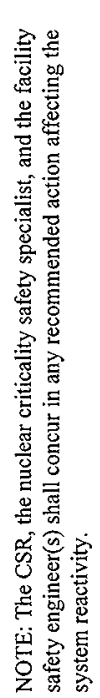 & 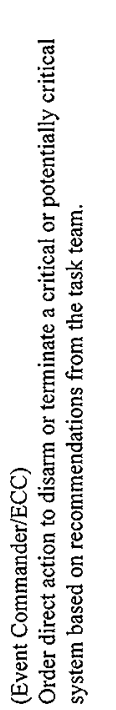 & 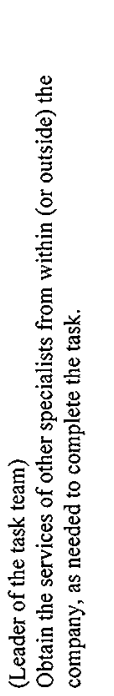 & 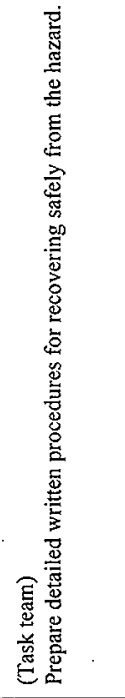 & 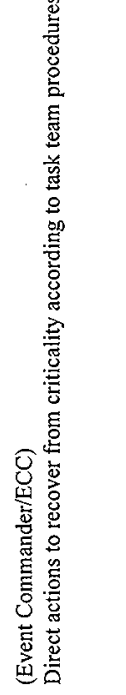 \\
\hline 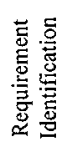 & 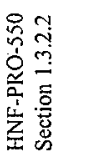 & & 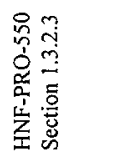 & 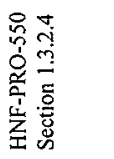 & 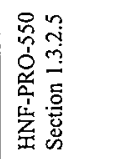 & 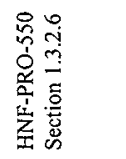 \\
\hline 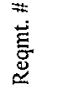 & $\begin{array}{l}\hat{0} \\
\text { ஜ } \\
\text { in }\end{array}$ & & $\begin{array}{l}\text { : } \\
\stackrel{0}{0} \\
\text { n. }\end{array}$ & $\begin{array}{l}\text { \&े } \\
\text { है }\end{array}$ & $\begin{array}{l}\stackrel{\circ}{\dot{D}} \\
\text { 足 }\end{array}$ & $\begin{array}{l}\overline{0} \\
\text { ह }\end{array}$ \\
\hline
\end{tabular}


TWR-3721 Rev. 0

This page intentionally left blank. 


\section{DISTRIBUTION SHEET}

To

Distribution

Project TitleMork Order

TANK WASTE REMEDIATION SYSTEM NUCLEAR CRITICALITY SAFETY PROGRAM MANAGEMENT REVIEW

\section{Name}

DESH

M. C. Brady Raap

D. R. Bratzel

R. J. Cash

T. C. Geer

M. A. Smith-Fewell LMHC

D. I. Aliten

C. B. Bryan

C. DeFigh-Price

M. P. DeLozier

W. T. Dixon

E. R. Hamm

H. R. Hopkins II

E. E. Mayer

N. J. Milliken

M. A. Rayne

D. N. Price

R. S. Popielarczyk

R. J. Shupe

L. E. Thomas

EDH

S. T. Almodovar

G. R. Franz

E. A. Geiger

PNL

A. W. Prichard

DOE

D. H. Alexander

WMH

T. S. Vail

CENTRAL EILES
From

NS\&L Criticality Support

\begin{tabular}{|c|c|c|}
\hline MSIN & $\begin{array}{c}\text { Text } \\
\text { With All } \\
\text { Attach. }\end{array}$ & Text Only \\
\hline
\end{tabular}

$\mathrm{R} 1-43$

$57-73$

57-73

R1-43

$\mathrm{R} 1-43$

$R 2-50$

R2-50

$\mathrm{R} 2-12$

[17 -07

R3-01

R1-3I

$\mathrm{R} 2-58$

R2-50

R2-50

$\mathrm{R} 2-58$

R3-01

$R 2-58$

$\mathrm{R} 2-50$

R3-01

A. $3-02$

S7-40

G6-60

$K 8-34$

$57-43$

T4-56

A.3-88
Page 1 of 1

Date April 8, 1999

EDT No. 625111

ECN No. NA

Attach.I

Appendix Only
EDT/ECN

Only 